Issued by Sandia National Laboratories, operated for the United States Department of Energy by Sandia Corporation.

NOTICE: This report was prepared as an account of work sponsored by an agency of the United States Government. Neither the United States Government, nor any agency thereof, nor any of their employees, nor any of their contractors, subcontractors, or their employees, make any warranty, express or implied, or assume any legal liability or responsibility for the accuracy, completeness, or usefulness of any information, apparatus, product, or process disclosed, or represent that its use would not infringe privately owned rights. Reference herein to any specific commercial product, process, or service by trade name, trademark, manufacturer, or otherwise, does not necessarily constitute or imply its endorsement, recommendation, or favoring by the United States Government, any agency thereof, or any of their contractors or subcontractors. The views and opinions expressed herein do not necessarily state or reflect those of the United States Government, any agency thereof, or any of their contractors.

Printed in the United States of America. This report has been reproduced directly from the best available copy.

Available to $\mathrm{DOE}$ and DOE contractors from

Office of Scientific and Technical Information

P.O. Box 62

Oak Ridge, TN 37831

Prices available from (703) 605-6000

Web site: http://www.ntis.gov/ordering.htm

Available to the public from National Technical Information Service

U.S. Department of Commerce

5285 Port Royal Rd

Springfield, VA 22161

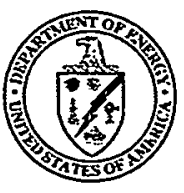




\section{DISCLAIMER}

\section{Portions of this document may be illegible in electronic image products. Images are produced from the best available original document.}


SAND99-3155

Unlimited Release

Printed January 2000

\title{
Z-Pinch Fusion for Energy Applications
}

\author{
Rick B. Spielman \\ Z-Pinch Physics and Power Flow Department \\ Sandia National Laboratories \\ P.O. Box 5800 \\ Albuquerque, NM 87185-1194
}

\begin{abstract}
$\mathrm{Z}$ pinches, the oldest fusion concept, have recently been revisited in light of significant advances in the fields of plasma physics and pulsed power engineering. The possibility exists for $z$-pinch fusion to play a role in commercial energy applications. We report on work to develop z-pinch fusion concepts, the result of an extensive literature search, and the output for a congressionally-mandated workshop on fusion energy held in Snowmass, Co July 11-23, 1999.
\end{abstract}





\section{Contents}

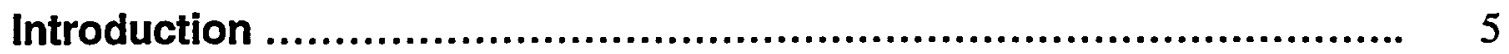

Description of LDRD Effort ..................................................... 5

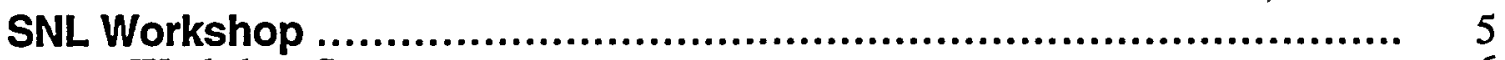

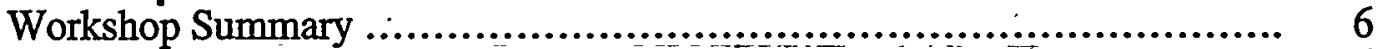

General Advantages of Z-Pinch IFE ............................................ 6

MITL Concepts .......................................................... 6

Standoff Designs ........................................................ 7

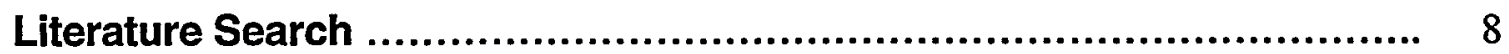

The Snowmass Workshop ........................................................ 8

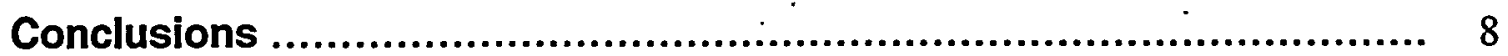

Appendix A - Workshop Presentations ..................................... 10

Appendix B - Literature Search ............................................... 229

Appendix C - Snowmass IFE Report .................................... 234 


\section{Introduction}

$\mathrm{Z}$ pinches are the oldest fusion energy confinement system. Recent advances in the fields of pulsed-power engineering and z-pinch physics prompted us to revisit imploding $\mathrm{z}$ pinches as a viable configuration for inertial fusion energy (IFE). Fundamentally, z pinches still are an attractive option for fusion energy. This optimism is based on the high efficiency of the z-pinch concept and the resulting low cost. In all fusion schemes the fundamental efficiency of the driver is coupled to the fusion output of the scheme and the efficiency of the output conversion system. It is this combined efficiency that is the most serious impediment to ALL fusion concepts.

We describe some preliminary work supported by Sandia National Laboratories with Laboratory Directed Research and Development (LDRD) funds (3535230000) to explore the possibility of using $\mathrm{z}$ pinches as the driver for fusion systems with a focus on energy applications.

\section{Description of LDRD Effort}

The intent of this work was to bring together experts in the field of fusion energy, to develop new ideas related to $\mathrm{z}$ pinches for fusion energy, and to explore the potential of $\mathrm{z}$ pinches as a realistic possibility for fusion energy. This approach was taken because $z$ pinches lack the diversity of ideas that have been developed over the last $30+$ years in other fusion energy concepts. In addition, they lack the long engineering history of the other major fusion energy concepts.

We had several broad goals for this effort. We needed to begin to develop the idea or concept base for z-pinch fusion energy concepts. We had to explore some of the key technical and costs issues that hitherto fore prevented $\mathrm{z}$ pinches from begin considered seriously. Finally, we had to ensure that the official fusion community recognized that $\mathrm{z}$ pinches had a role in the national fusion effort.

In order to achieve these goals we held a Workshop at Sandia to explore z-pinch fusion issues, we conducted a major review of the fusion literature, and we participated in a DOE fusion study that explored future fusion options. These efforts are documented herein.

\section{SNL Workshop}

A workshop was held on April 27-28, 1999 at Sandia National Laboratories and was attended by $\sim 20$ people from national laboratories, universities, and private industry. We reviewed the status of $\mathrm{z}$ pinches and then explored the issues that are crucial for the development of economical fusion energy. The role of $\mathrm{z}$ pinches in this area was explored and concepts were described and developed. A number of feasible concepts were identified during the Workshop and marked for further study during the year by SNL personnel and University researchers. A final report was prepared and all of the viewgraphs that were presented were collected and then distributed to all of the attendees. Appendix A contains a copy of the presentations of the workshop.

The following attendees were present: (SNL) Rick B. Spielman, Stephen A. Slutz, Michael Cuneo, Mark Derzon, David L. Johnson, Ray Leeper, M. Keith Matzen, Michael Mazarakis, Dillon H. McDaniel, Craig Olson, Richard E. Olson, Tim J. Renk, Gary 
Rochau, Kenneth Struve, William A. Stygar; (LANL) Irv Lindemuth, Kurt Schoenberg, John Scott, Richard Seimon, Ronald Kirkpatrick,; (LLNL) James Hammer, L. John Pekins, Dmitri Ryutov; (NRL) Gerald Cooperstein; (Imperial College) Malcolm Haines; (Fusion Power Associates) Steve Dean; (University of Wisconsin) Robert Peterson, Gerald Kulcinski; (Consultant) Marshall Sluyter; (Cornell University) John Greenly; (General Atomics) Dan Goodin; (University of California, Davis) John S. DeGroot; (University of California, Berkeley) Per Peterson; (Ohio State University) Peter Turchi.

\section{Workshop Summary}

A large number of new ideas came from the Workshop. It proved once more that putting a few good people together is a very good way to get new ideas or to flesh out older ideas. The Workshop identified two modes of potential z-pinch IFE operation: the first mode uses conductors of some form to deliver electrical energy to the load and, the second mode uses some sort of indirect method to deliver electrical energy to the load in a way to minimize the mass of electrodes (ion beams or kinetic projectiles). The participants clearly identified overall reactor efficiency as THE real concern (for any IFE design). Any loss in coupling or conversion efficiency impacts the overall design requirements very strongly. This in itself may rule out lower-efficiency ion beam variants. The overall opinion of the participants was that the efficiency of pulsed power is the real attraction of these concepts.

Perhaps, not too surprisingly, there was the general feeling that MFE concepts such as Tokamaks have just as many engineering problems as pulsed power systems. They have just had the advantage (or misfortune) of being studied more intensely for a longer period of time.

The general conclusions of the participants of the Workshop were: low mass disposable MITL concepts were feasible, that low repetition rates were desirable $(\sim 0.1 \mathrm{~Hz})$, the target chamber engineering was not a problem, the cost of technology demonstration systems would be cheaper than MFE alternates, and the high yield targets ( $>1 \mathrm{GJ}$ ) were needed.

\section{General Advantages of Z-Pinch IFE}

Why are we considering z-pinch IFE concepts? The key reasons (related) are cost, efficiency, and simplicity. One trades the incredibly complex system of a Tokamak or a laser with the simplicity of pulsed power. Compare the efficiency of NIF with that of Z.Z can deliver $x$ rays with $15 \%$ efficiency while the efficiency of NIF is less than $1 \%$. Consider the transport physics and target constraints of a laser (or heavy ion) system with the almost crude vacuums needed for pulsed power. With the exception of standoff (described below) pulsed power drivers remove the risk from the reactor engineering systems and place it at the load. This is almost exactly opposite other MFE and IFE concepts in which the risk is in the facility and subsystems. Think about the difficulty of designing long life components in a Tokamak radiation environment.

\section{MITL Concepts}

If there is a single perceived negative for $\mathrm{z}$ pinches for IFE it is the issue of standoff. A large yield detonation would destroy or damage large amounts of MITL material. The 
cost (and activation) of that material becomes a real problem. The Workshop identified over and over the "Kopeck" problem that is common to ALL IFE concepts. That is the cost of disposable hardware and targets must be low enough to be cost effective. It was noticed that the reprocessing and storage of radioactive waste is a cost factor. A major result of the Workshop was the identification of two possible MITL configurations for IFE reactors.

The first MITL approach was to use a MITL material that was useful in some way and not subject to activation. Dr. Per Peterson of UC Berkeley suggested using $\mathrm{Li}_{2} \mathrm{BeF}_{4}$ (called Flibe) as the base material for the MITLs. This material could be cast or injection molded into the shape of MITLs. If necessary a thin conducting layer could be sprayed on the surface to improve electrical and vacuum properties. During the high yield energy release the Flibe would absorb energy from the neutrons and heat to its melting point. It would then drop to the reactor floor and mix with the Flibe liquid coating the walls. It would then be pumped out to the heat exchanger. Activation is minimized and the energy absorbed by the Flibe is a great way of absorbing the neutrons. The issue here is the mechanical properties of the Flibe and its ability to be formed into the necessary shapes.

The second MITL approach was suggested by Dr. Jim Hammer of LLNL. Here the motivation was to minimize the mass of the MITLs but to use a normal material such as aluminum. In this design the solid MITLs would be replaced by an array of thin wires. The wires would have a thickness such that they would not vaporize. during the current pulse. The ability to make an array of wires and to deform the array into the conical shape required looks relatively straightforward. The mechanical tolerances needed for the MITLs with such a system could be easily met.

Other more speculative designs were discussed including liquid electrodes but were thought to be too far from reality at this time to be considered seriously.

\section{Standoff Designs}

It is always attractive to think about getting standoff using an indirect charging technique to drive the pinch. You eliminate all of those nasty MITL concerns - at the expense of efficiency. The trade off here is critical. And yet with indirect systems there are still MITL components. There is still an electrical circuit. You are simply restricting the mass of MITL material that will be destroyed. Two approaches were discussed at the Workshop.

One concept, described by Gerry Cooperstein of NRL, used ion beams and an inverse diode to feed electrical power to the load. A proton beam with a peak energy of $\sim 5 \mathrm{MeV}$ was used to deliver current to a z-pinch load. The positive attributes of this concept are stand off, demonstrated proton beam performance, and modest divergence requirements. The problem is that no inverse diode has been demonstrated near these performance levels. If one examines the efficiency of ion diodes and the projected efficiency of an inverse diode one is lucky to get even $10 \%$. The further efficiency of coupling to the load and generating $\mathrm{x}$ rays is lower still. There are other concerns that include vacuum power flow in the plasma filled MITLs.

The second approach uses high velocity projectiles to deliver the kinetic energy. For this concept electromagnetic launchers accelerate a projectile to $>10 \mathrm{~km} / \mathrm{s}$. A second, "catcher" mass can either be injected at high velocity or dropped into the path of the main projectile. The catcher carries a strong imbedded magnetic field. This field is compressed and generates a load current. Some form of pulse forming would be required as the rise 
time of this primary current would be too slow for the pinch. There are numerous advantages. The catcher and/or projectile can be made from pure lithium or a lithium alloy and could stop nearly all of the neutrons.

\section{Literature Search}

A comprehensive literature search in the topical area of Z-pinch fusion reactors was conducted. Not only was the Sandia National Laboratories' technical library involved but, in addition, personal contacts were made with active and retired researchers around the world to find obscure articles and publications. The search was completed and the articles were indexed and compiled and then made available to interested parties around the world. A list of the obtained articles is given in Appendix B.

We found that many articles had been written in the field of z-pinch fusion reactors over the years. The rationale given in these articles parallels that of this report. The simplicity and the efficiency of $\mathrm{z}$ pinches makes them attractive even if plasma instabilities are a significant problem.

\section{The Snowmass Workshop}

The US Congress mandated that the DOE hold a Workshop to discuss the status of the national Fusion Energy Program. The conference was held in Snowmass, Colorado, July 11-23, 1999. As part of that Workshop Sandia sent a number of participants. Besides presenting technical reports describing the state of z-pinch and inertial confinement fusion (ICF) target research at SNL we lead the effort to write the report that summarized the relationship of Inertial Confinement Fusion to the National Fusion Energy Program. That ICF report is included in full in Appendix C.

The Snowmass Workshop was intended to cover the entire field of fusion for energy applications. This includes magnetic fusion concepts (MFE), inertial fusion concepts (IFE), and other innovative concepts that do not fit into those labels. The earlier z-pinch workshop held at Sandia National Laboratories laid the groundwork for our input in the IFE Subgroup of the Snowmass Workshop. We were able to have significant input to the IFE Subgroup final report. (See Appendix C.)

The final report of the IFE Subgroup stated that $z$-pinch IFE concepts were scientifically feasible. The next step would be further work in the field of z-pinch fusion energy funded at the level of concept exploration by DOE Office of Fusion Energy.

\section{Conclusions}

The goals of the work defined by the LDRD were substantially met or exceeded. These were:

1. Hold a Workshop at Sandia National Laboratories on the topic of $\mathrm{z}$ pinches for fusion energy. Invite a wide-ranging group to discuss the possibilities. Develop z-pinch fusion energy concepts for further development.

2. Review the literature in the area of $\mathrm{z}$ pinches for fusion energy and compile all of the articles found on the topic. 
3. Have significant Sandia participation in the DOE-sponsored Snowmass Workshop on fusion energy. Participate in the development of national policy for inertial confinement fusion for fusion energy.

We conclude that $\mathrm{z}$ pinches are a viable option in the national fusion energy program. While not presently a mature technical effort the field of $\mathrm{z}$ pinches for fusion energy warrants additional funding to determine the feasibility of the concept.

We are proceeding with plans to obtain Phase 1 Emerging Concepts level funding from the DOE Office of fusion energy to further explore the possibilities that have been discovered in this initial work. 


\section{Appendix A - SNL Workshop Presentations}


UCRL-JC-126188

PREPRINT

\section{A User Facility for Research on Fusion Systems with Dense Plasmas}

\section{D.D. Ryutov}

This paper was prepared for submittal to 2nd Symposium on Current.Trends in Intemational Fusion Research:

Review and Assessment

Washington, DC

March 10-14, 1997

January 7, 1999

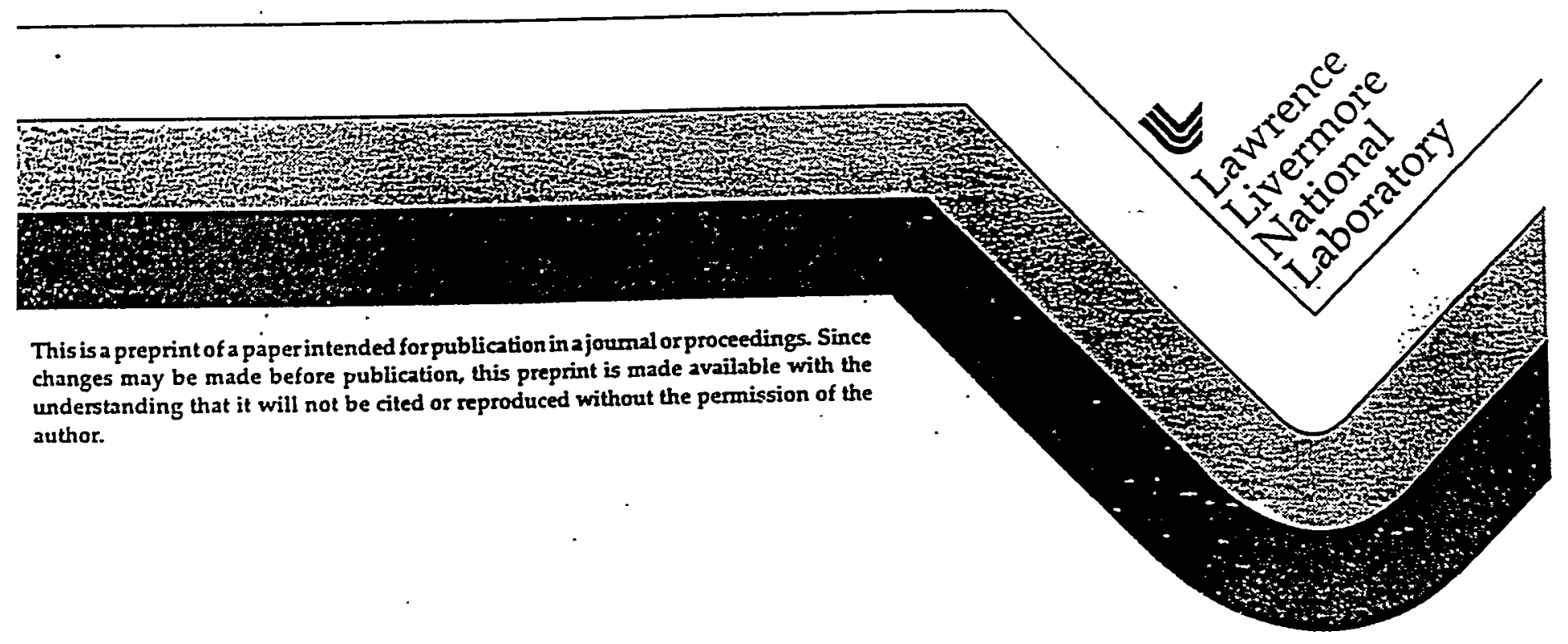




\title{
A USER FACILITY FOR RESEARCH ON FUSION SYSTEMS WITH DENSE PLASMAS
}

\author{
Dmitri D. Ryutov \\ Lawrence Livermore National Laboratory, Livermore, CA 94551, USA
}

\begin{abstract}
There are fusion systems whose dimensions could be scaled down to a few centimeters, if the plasma density and confining magnetic field are raised to sufficiently high values. These systems include the FRC, spheromak, Z-pinch, multiple mirrors, etc. The fusion-grade plasma in these systems can be obtained with the energy deposited to the plasma as low as $10-100 \mathrm{~kJ}$. This prompts a "user-facility" approach to the studies of this class of fusion systems. The user facility would consist of a pulsed energy source (presumably, a Marx generator), and a set of diagnostics permanently deployed at the facility site. Research groups could bring their own "targets" (experimental assemblies of a few centimeter size) to perform series of experiments at the facility. Because of their small size, the targets will be relatively inexpensive and thus well within the reach of the university groups. The paper describes several specific experiments on creation and subsequent adiabatic compression of centimeter-size closed field line configurations. We also discuss possible development of stand-off energy sources suitable for pulsed fusion reactors with such targets.
\end{abstract}

\section{Introduction}

There are a number of fusion systems whose dimensions can be scaled down to a few centimeters; if the plasma density and confining magnetic field are raised to sufficiently high values. These systems include the field-reversed configuration (FRC), spheromak, Zpinch, multiple mirrors, and some others. The fusion-grade plasma in these systems can be obtained with the energy deposited to the plasma as low as 10-100 kJ. This prompts a "user-facility" approach to the studies of this class of fusion systems. The concept of such a user facility was first briefly mentioned in Ref. 1 . Here we present a more detailed description.

The user facility would consist of a pulsed energy source (presumably a Marx generator) and a set of diagnostics permanently deployed at the facility site. Research groups could bring their own "targets" (experimental assemblies of a few centimeters size, see below for some specific examples) to perform a series of experiments at the facility. Because of their small size, the "targets" would be relatively inexpensive, and thus well within the reach of the university groups. The concept and design of the targets would be entirely the mission of the participating groups. The availability of such a user facility for 
performing experiments should have a strong favorable effect on the creative potential of fusion research.

The main energy source could be a Marx generator with the stored energy up to 1 $\mathrm{MJ}$ and the pulse-length in the microsecond range. Certain pulse-shaping capacity would increase the range of possible experiments. A couple of separate energy sources with the energy content up to $100 \mathrm{~kJ}$, for generating the bias magnetic field, would be desirable. The interface of the Marx generator and the load can be made very flexible.

The set of diagnostics should include optical, UV, and x-ray spectroscopy; neutron diagnostics (for both DD and DT neutrons, with tritium used as a trace element); optical imaging; and $x$-ray backlighting. The typical time-scale of plasma processes for the aforementioned fusion systems will not be shorter than 30-50 ns. Particular research groups might also provide their own diagnostics.

There is a similarity between the concept of a user facility presented in this paper and the concept of international center for the studies of plasma focus presented by Herrera [2]. Perhaps, a combination of the two would rise the chances for the actual creation of such a facility. There already exist examples of large facilities which serve, at least in part, as user facilities. The Nova laser in the Lawrence Livermore National Laboratory is used by a numerous university groups; the $\mathbf{Z}$ facility at Sandia National Laboratories (fast $\mathbf{Z}$ pinch) is a site of a number of collaborative multi-institutional experiments. The proposed facility would allow research groups with limited resources to contribute in a significant way to development of several fusion concepts.

\section{Examples of possible experiments}

To be more specific, we consider in more detail two possible experiments related to the FRC. The first is the study of the formation and properties of an FRC with the density $n \sim 10^{18} \mathrm{~cm}^{-3}$, the temperature $T \sim 100 \mathrm{eV}$, and the magnetic field strength $B \sim 100 \mathrm{kG}$. This set of parameters corresponds to

$$
\beta \equiv \frac{16 \pi n T}{B^{2}} \sim 1 .
$$

The radius of the FRC can be $a \sim 1 \mathrm{~cm}$, and the length $L \sim 4-6 \mathrm{~cm}$. Such an object could then be adiabatically compressed by an imploding liner (see Ref. 3 for a more detailed discussion and further references).

Magnetic coils of a radius $\sim 1.5 a$ would be used for creating the bias magnetic field and for the field reversal. The bias coil can have a relatively long pulse, up to a hundred microseconds. There should not be any problems in creating such a coil. The field-reversal coil should be turned on within a time of order of several axial Alfven transit times [4],

$$
\tau=\alpha L / \mathrm{v}_{A} \text {, }
$$


with $\alpha$ being of the order of 2 . For the aforementioned set of parameters, and for a deuterium plasma, one has $\tau-1 \mu \mathrm{s}$. This estimate sets the time-scale for the controlled changes of the magnetic field.

The total energy content in the plasma will be $\sim 1 \mathrm{~kJ}$, and the magnetic energy will be several times higher, $\sim 3 \mathrm{~kJ}$ (because the magnetic field occupies a larger volume). For $\tau \sim 1 \mu \mathrm{s}$, the power level involved into the process of field reversal will be $\sim 3 \mathrm{GW}$. The current in the coil,

$$
I \sim \frac{c B L}{4 \pi}
$$

should be $\sim 1.5 \cdot 10^{15} \mathrm{CGS} \sim 0.5 \mathrm{MA}$ (for $B \sim 100 \mathrm{kG}$ and $L=6 \mathrm{~cm}$ ). The required loop voltage will be of the order of $7.5 \mathrm{kV}$. All these parameters are not very demanding and can be reached even without use of the main energy source. In this experiment, the pulsed magnetic system can possibly be designed so as to survive multiple shots.

At the temperature of $100 \mathrm{eV}$, the plasma will be fully ionized, and its radiative losses will be [5]:

$$
P_{\text {rad }}(W)=1.7 \cdot 10^{-32} n^{2}\left(\mathrm{~cm}^{-3}\right) T^{1 / 2}(\mathrm{eV}) \cdot \pi a^{2}(\mathrm{~cm}) L(\mathrm{~cm})
$$

For the parameters given above, this power will be only $2.5 \mathrm{MW}$, much less than the total power delivered to the plasma during the reconnection event, $1 \mathrm{~kJ} / 1 \mu \mathrm{s} \sim 1 \mathrm{GW}$. This means that radiative losses from a pure plasma are negligibly small. For radiative losses to become considerable, the plasma should become very dirty, with the amount of heavy impurities (of the type of iron) in the range of $1 \%$.

The FRC with the aforementioned parameters will have a ratio of plasma radius to a characteristic ion gyro-radius of $-30-50$, much higher than in the existing experiments and very close to the values of this parameter expected for a FRC-based fusion reactor [4].

If successful, this experiment will pave the road to the second one, where the preformed FRC will be translated into an imploding liner of the type described in Ref. 3 and then adiabatically compressed. We conceive of a scenario where the on-axis hole through which the FRC will be injected will be closed early in the implosion, thereby trapping the FRC inside the liner. This can be achieved by using a liner whose linear density (mass per unit length) on the injection end is smaller than over the rest of its length (Cf. Ref. 6)

The compression should be 3-dimensional, because in 3D implosions the energy is delivered predominantly to the plasma, not to the embedded magnetic field (Cf. Ref. 3). The feasibility of quasi-spherical implosions has been demonstrated in the experiments by Degnan et al (Ref. 7). In geometrically self-similar 3D implosions, the plasma temperature scales as

$$
T=T_{0} C^{2}
$$

where $C$ is a linear convergence (the ratio of the initial dimension to the instantaneous dimension). If one starts with the plasma with the temperature $T_{0}=100 \mathrm{eV}$, the fusion-grade plasma needs reaching $C \sim 7-10$. Note that, in the aforementioned experiments by Degnan et al, the maximum linear convergence was close to 7 . According to the analysis carried out 
in Ref. 3, the life-time of the hot dense state is determined by the liner expansion under the action of the plasma pressure. For the liners with a mass of a few grams, one can obtain the fusion gain $Q \sim 1$. The energy delivered to the liner should be in the range of $100-200 \mathrm{~kJ}$. Assuming that the efficiency of the energy transfer from the condenser bank to the liner is $\sim 10 \%$, one sees that the energy stored in the condenser bank should be 1-2 MJ. This sets the scale for the main energy source for the user facility.

The experimental assembly will now have to be replaced after every shot (for this reason, we have used the word "target" to designate the experimental assembly). But since the target is compact (with the maximum dimension not exceeding 10-15 cm), manufacturing a few dozen targets for one experimental campaign should be inexpensive. We imply that the experimental group will bring these targets to the user facility and "shoot" them out within a couple of weeks.

\section{Diagnostics issues.}

The possibility of providing a set of sophisticated diagnostics permanently deployed at the user facility is one of the most attractive features of the user facility approach (the diagnostics constitute, possibly, the most expensive part of the hardware). For the configurations where the plasma is not obscured by the liner, one can use spectroscopy to measure relative line intensity, Stark broadening, Doppler broadening, possibly with a deliberate adding of trace impurities. The electron density and temperature could be measured by Thomson scattering. Faraday rotation could be used for the measurements of the magnetic field. Optical and UV imaging could be used to view an overall structure of the plasma configuration. For the configurations obscured by the liner, one could perform $\mathrm{X}$-ray imaging (provided the liner material is transparent in the chosen spectral band) as well as detect fusion neutrons. X-ray backlighting could be used to observe the interface between the plasma and the liner. In some cases (in particular, if one implodes a mirror-like configuration), the presence of the liner would still allow using axial viewing ports.

\section{Development of stand-off energy sources}

An important part of the activities centered around the user facility, could be development of stand-off energy sources for pulsed fusion devices based on liner implosions. A problem with such devices is that, in every implosion, a large amount of energy is released from the fusion plasma. If implosion occurs at the tips of the electrodes of the power supply system, an unacceptable thermo-mechanical damage to an expensive hardware occurs. Certainly, a commercial fusion reactor cannot operate in such a mode.

A possible way of solving this problem was delineated in Ref. 3, where it was suggested that the fusion reactor would work in the following way: the experimental assemblies will be dropped into explosion chamber (whose walls would be protected by 
liquid lithium, very much as in ICF reactors, Ref. [8]), and the energy required to drive the implosion will be delivered from the distance of tens of meters (see below).

It is assumed that the assembly in this case will contain the following elements: i) the system for pre-forming the FRC (or other configuration to be adiabatically compressed); ii) the liner; iii) the on-board circuitry required to energize various systems in a required sequence (formation of pre-plasma, translation it into the liner, liner implosion).

There are at least two ways of delivering the energy to the assembly dropped into the explosion chamber. The one is to use the "inverse diode" system [3], where the assembly would be energized by an $1-\mathrm{MeV}$ electron beam, penetrating through the entrance foil, being absorbed by a cathode, and generating a voltage between the foil and the cathode. With an appropriate circuitry (including, possibly, a pulse transformer) installed in the assembly, this energy source could be used to drive some fast circuits. The second way is based on the use of fast flyers accelerated either electromagnetically (Ref. 9) or explosively (Ref. 10). These flyers could then be used to compress the conducting flux conserver enclosing some seed magnetic field (which could be generated, in particular, by the inverse diode system). The kinetic energy of the flyer would be converted into the magnetic energy and the latter would drive a circuit of the imploding liner. The flyers with velocities of order of $10^{7} \mathrm{~cm} / \mathrm{s}$ were obtained in electromagnetic accelerators, with the flyer energy $\sim 100 \mathrm{~kJ}$ [9]. Explosively driven cumulative jets with velocities up to $9 \cdot 10^{6} \mathrm{~cm} / \mathrm{s}$ were obtained [10].

The studies of detached energy sources are at present in their infancy, and the contribution of the groups working at the user facility could be very important. All the diagnostics deployed for the studies of plasma physics issues can be used effectively also for the studies of operation of the prototypical energy conversion systems (inverse diode, magneto-explosive generators, etc).

\section{Discussion}

The 3D liner implosions can also be used to adiabatically compress some other closed-field-line configurations, like the spheromak and even a spherical tokamak (with a central post mounted inside the liner). Although an imploded spherical tokamak may not have a great future as a fusion reactor, it may allow reaching a.new parameter domain in terms of plasma density and thereby add substantial new information to the physics of tokamaks.

Purely radial liner implosion on a multimirror system with a dense plasma would provide favorable experimental conditions for the studies of the wall confinement (see Ref. 11) - an issue of great importance for many pulsed systems with dense plasmas, including MAGO [12]. The advantage of the open-ended system is the presence of an axial access.

All in all, creation of a user facility for studies of pulsed systems with a dense plasma would add a niew dimension to fusion research. It would give an opportunity to 
numerous university research groups to fully develop and demonstrate their creative potential. It would also be an excellent way to build up an international collaboration on innovative fusion concepts.

\section{Acknowledgment}

This work was performed under the auspices of the U.S. Department of energy by Lawrence Livermore National Laboratory under contract W-7405-Eng-48.

\section{References}

1. D.D. Ryutov. "Reinventing Fusion." Plenary talk at 12th American Nuclear Society Topical Meeting on the Technology of Fusion Energy, Reno, NV, June 16-20, 1996.

2. J. Herrera. "The International Center for Dense Magnetized Plasmas." This Symposium.

3. R.P. Drake, J.H. Hammer, C.W. Hartman, L.J. Perkins, D.D. Ryutov. "Submegajoule Liner Implosion of a Closed Field Line Configuration." Fusion Technology, 30, 310 (1996).

4. M. Tuszhewski. "Field Reversed Configurations." Nucl. Fusion, 28, 2033 (1988).

5. J.D. Huba. NRL Plasma Formulary, NRL/PU/6790-94-265, 1994.

6. S.G. Alikhanov, S.G., V.P. Bakhtin, V.M. Brusnikin, I.S. Glushkov, R.Kh. Kurtmullaev, A.L. Lunin, A.D. Muzychenko, V.P. Novikov, V.V. Pichugin, V.N. Semenov, G.E. Smolkin, E.G. Utyugov, I. Ya. Shipuk (1977). "Investigation of model thermonuclear systems with liners." In Proc. 6th Conf. on Plasma Phys. and Contr. Nucl. Fusion Res., Berchtesgaden Germany, October 6-13 1976, Vol. 3, p. 517, International Atomic Energy Agency, Vienna, 1977.

7. J.H. Degnan et al (25 co-authors). "Electromagnetic Implosion of Spherical Liner". Phys. Rev. Lett., 74, 98 (1995). J.H. Degnan, This Conference.

8. R. Moir. "The High-Yield Lithium-Injection Fusion-Energy (HYLIFE)-II Inertial Fusion Energy (IFE) Power Plant Concept and Implications for IFE." Phys. Plasmas, 2, 2447 (1995).

9. J.E. Osher, G. Barnes, H.H. Chau, R.S. Lee, C. Lee, R. Speer, and R.C. Weingart. "Operating Characteristics and Modeling of the LLNL 100-kV Electric Gun." IEEE Trans. Plasma Sci., 17, 392 (1989).

10. M.A. Lavrent'ev and B.V. Shabat. "Hydrodynamical Problems and their Mathematical Models," Moscow, Nauka Publishing House, 1973 (in Russian).

11. D.D. Ryutov. "Controlled Thermonuclear Fusion in a Dense Quasistationary Plasma." Sov. Phys. Uspekhi, 18, 466 (1975).

12. R.C. Kirkpatrick. "Magnetized Target Fusion (MTF): Relaxing the Driver Requirements," This Symposium. 


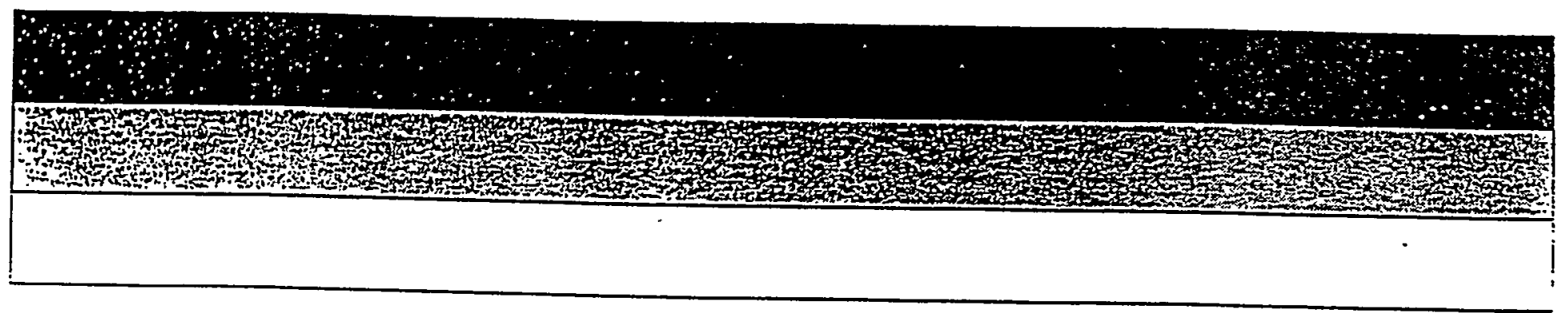

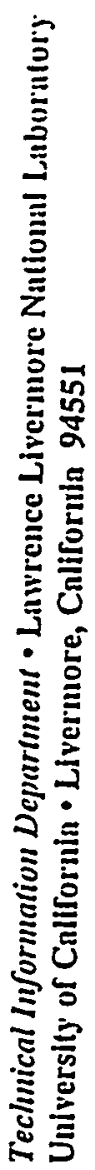




\section{SUBMEGAJOULE LINER IMPLOSION OF A CLOSED FIELD LINE CONFIGURATION}

\author{
R. PAUL DRAKE, JAMES H. HAMMER, CHARLES W. HARTMAN, \\ L. JOHN PERKINS, and DIMITRI D. RYUTOV* \\ Lawrence Livermore National Laboratory, Livermore, California 94550
}

Received April 28, 1995

Accepted for Publication March 4, 1996
PLASMA ENGINEERING

KEYWORDS: fusion high-density plasma, pinches
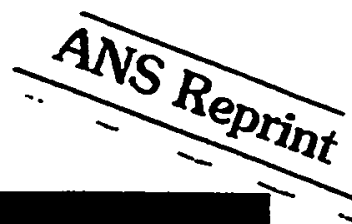

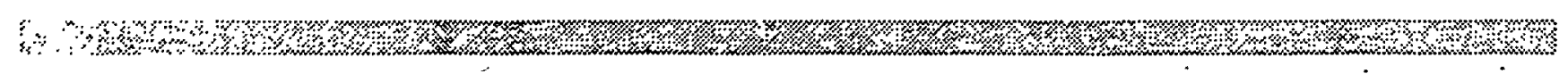

Adiabatic compression of a preformed closed field line configuration by an imploding liner is considered. Three configurations are discussed: the field-reversed configuration, the spheromak, and the Z-pinch. It is shown that by employing a two-dimensional compression, one can reach a breakeven condition with an energy input into the plasma as low as $1.00 \mathrm{~kJ}$. Typical initial dimensions of the liner are length, $5106 \mathrm{~cm}$; radius, $-1 \mathrm{~cm}$; and wall thickness, $\sim 0.01 \mathrm{~cm}$. Liner mass is in the range of a few grams. It is assumed that the initial plasma beta is of the order of unity; in this case, the final beta is much greater than $I$, and the plasma is in a wall confinement regime. Typical plasma parameters for the final state (for the linear compression ratio equal to 10) are density, $10^{21} \mathrm{~cm}^{-3} ;$ temperature, $10 \mathrm{keV}$; and magnetic field, $10^{7} \mathrm{G}$. A brief discussion of various phënomena affecting the wall confinement is presented (magnetic field diffusion, radiative losses, and impurity penetration); the conclusion is drawn that the heat losses to the walls are modest and are not a factor that limits plasma enhancement Q: It is shown that at least for relatively thin liners, whose compressibility can be neglected, what limits $Q$ is a relatively short liner dwell time near the maximum:compression point. The scaling law for the $Q$ versus the input parameters of the system is derived, which shows a relatively.weak dependence of $Q$ on the input energy. Possible ways for increasing the dwell time are discussed. Reactor potentialities of the system are briefly described. It is emphasized that the possibility of performing crucial experiments on small- to medium-scale experimental devices may considerably-shorten the development path for the system under consideration. Some nonfusion applications of the system described are mentioned. Among them are buming and transmutation of longlived fusion products, medical isotope production, a pulsed source of hard $X$ rays, and fusion neutrons.

\section{INTRODUCTION}

During the last decade, considerable progress has been made in developing a technique for imploding liners (see, e.g., Ref, 1). This technique at present allows the deposition of the order of $1 \mathrm{MJ}$ of energy into liners with an initial diameter of the order of a few centimetres and a mass of a few grams. It is well known that the imploding liners can serve to adiabatically compress a preformed magnetized plasma. This approach was pursued in the 1970 s by several research groups. The magnetic configurations included a linear geometry with cusp end plugs, ${ }^{2,3}$ a $Z$-pinch, ${ }^{4}$ and the field-reversed configuration (FRC) formed either by the plasma currents ${ }^{5}$ or by a rotating relativistic electron beam. ${ }^{3}$ The range of param-

*Permanent address: Budker Institute of Nuclear Physics, Novosibirsk 630090 . Russia. eters considered in these studies corresponds to comparatively large dimensions of the system and accordingly to large required energy inputs in the range of tens and even hundreds of megajoules. A nice survey of this earlier work has been given by Ribe and Sherwood. ${ }^{6}$

In the present paper, we revisit the problem, having in mind smaller initial dimensions, shorter implosion times, and smaller input energies, in the range of 0.01 to $1 \mathrm{MJ}$, and initial liner length, radius, and weight, in the ranges of 5 to $10 \mathrm{~cm}, 1$ to $2 \mathrm{~cm}$, and 5 to $10 \mathrm{~g}$, respectively. We consider an option provided by a self-similar implosion of the liner in both $r$ and $z$ directions and show that this mode of implosion has advantages over purely radial implosion (in what follows, we call this $r-z$ mode a two-dimensional implosion; we call a purely radial mode a one-dimensional implosion). We consider only closed field line configurations, which would provide good plasma thermal insulation from the liner and the end walls; 
we try to find conditions under which the fusion alpha particles would also be confined. We concentrate on the situation in which the initial plasma (which is to be adiabatically compressed by the imploding liner) has a pressure comparable with or exceeding the initial magnetic pressure of the magnetic field inside the liner, so that essentially all the energy of the implosion is spent on the plasma heating and not on the increase of the energy of the magnetic field immersed in the plasma. What we find is that with all these ingredients combined together, it is probably possible to achieve a plasma density of $\sim 10^{21}$ $\mathrm{cm}^{-3}$ and fusion breakeven $(Q=1)$ at a plasma energy level of $\sim 0.1 \mathrm{MJ}$, while values of $Q$ as high as 5 to 6 are probably within the reach of the experiments with a plasma energy content of $-1 \mathrm{MJ}$.

In the same range of input energies, another compact pinch system may work: This is the so-called staged $Z$-pinch (see Ref. 7 and references therein). A staged $Z$-pinch is supposed to use considerably lighter (e.g., gaseous) liners and to obtain much higher final densities $\left(-10^{24} \mathrm{~cm}^{-3}\right)$. The physics involved may be considerably different, but the required experimental hardware is very similar, so that two sets of liner experiments can be, in principle, carried out on the same device.

Magnetically insulated liner concepts, such as the concept discussed in this paper, are also referred to as. magnetized target fusion ${ }^{8}$ (MTF) (see also Ref. 9). A highenergy (many tens of megajoules) liner/MTF variant is the MAGO device under study at Los Alamos National Laboratory and the All-Russian Scientific Research Institute of Experimental Physics. ${ }^{10}$

The present paper by no means pretends to offer a solution to the problem of a compact fusion reactor. It just allows us a clearer delineation of what we think is a promising parametric domain for the further theoretical and (possibly) experimental studies and to identify key physics uncertainties yet to be resolved. Still, as there are some chances that the system that we are considering will reach high- $Q$ regimes, we include a discussion of the reactor issues specific for pulsed liners (Sec. VIII).

The paper is organized as follows: In Sec. II, we present a general outline of the concept and specify the closed field line configurations that will be discussed in the paper. In Sec. III, we consider the scaling laws for a two-dimensional adiabatic compression of a plasma with an initial beta value of the order of unity. We find that in the course of a two-dimensional adiabatic compression, the beta value becomes considerably $>1$. Therefore, a regime of so-called wall confinement "should be realized in our system, with a magnetic field inside the liner serving primarily to suppress plasma thermal conductivity to the walls. In Sec. IV, we briefly analyze the motion of the liner. In Sec. V, we present parameters of a conceivable experiment. In Sec. VI, we consider the issues of plasma thermal conductivity. We come to the conclusion that even for thermal conductivity that is only by a factor of a few less than the Bohm thermal conductivity, the resulting confinement time does not limit the attainable values of the plasma enhancement $Q$. What, in fact, limits $Q$ is the liner dwell time near the point of maximum compression: Because of finiteness of the liner inertia, this time turns out to be relatively short. The dynamics of the system near the liner tuming point is discussed in Sec. VII; we present here a scaling of plasma $Q$ versus the input energy; we speculate on the possibility of some increase of the dwell time by ablating some amount of the liner material. Section VIII is devoted to reactor aspects of the scheme under consideration and possible nonfusion applications of this scheme. In Sec. IX, we briefly describe some physics problems for further analysis. Finally, Sec. X contains a summary of our findings.

\section{GEHERAL DESCRIPTION OF THE COHCEPT}

A possible geometry of the system is illustrated by Fig. 1, which depicts a situation where the magnetic configuration is of the FRC type, with the field reversal maintained by plasma currents (not by an electron or ion beam). Other possible configurations are discussed later.

We characterize the initial state of the FRC by its initial beta value

$$
\beta_{0}=8 \pi p_{0} / B_{0}^{2} .
$$

Here and in the following discussion, the subscript 0 denotes the initial values of the corresponding quantities. As we are going to restrict ourselves to only order-ofmagnitude estimates, we do not precisely specify the point in which $p_{0}$ and $B_{0}$ entering Eq. (1) are taken: This is just some characteristic point inside the FRC.

As is well known, initially a magnetically confined FRC has $\beta_{0} \sim 1$ as a natural state (see, e.g., survey, Ref. 12). For the system under consideration, with the confinement volume restricted on all sides by conducting

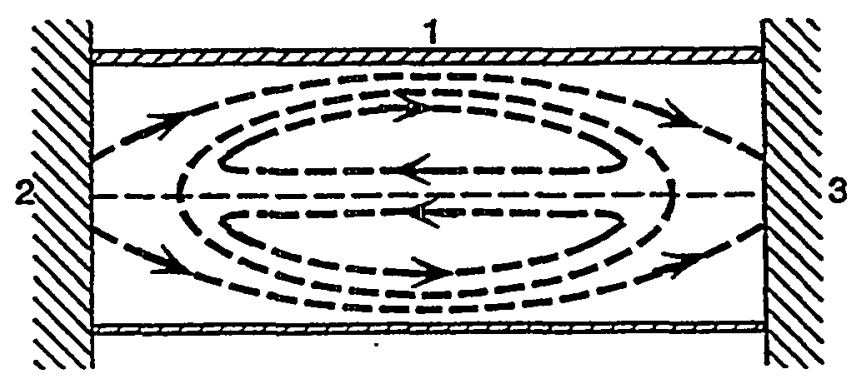

Fig. 1. A schematic showing the geometry of the imploaingliner system. The liner (1) will implode to adiabatically compress the plasma and the closed magnetic field configuration. Our reference case of an FRC is shown by the dashed lines. Structures 2 and 3 are the electrodes that drive the current that implodes the liner. 
material walls, an FRC configuration with $\beta_{0}>1$ is also possible. Therefore, we consider $\beta_{0}$ as a free parameter that is subject to the constraint

$$
\beta_{0}>1 \text {. }
$$

Plasma states with $\beta_{0}<1$ are impossible for the FRC because then the tension of the magnetic field lines would shorten the plasma volume in the axial direction until the $\beta_{0} \sim 1$ state is reached.

After the initial configuration is prepared, an external voltage is applied between electrodes 2 and 3 (Fig. 1) with a pulse-length short compared with the liner skin time. The axial current is then excited only in the liner and does not penetrate into the plasma volume. The current must be large enough that the pressure of the azimuthal $\varphi$ component of the driven magnetic field considerably exceeds (at least by a factor of a few) the initial plasma pressure inside the liner. Then the liner gets ac: celerated in the inward direction and stores considerable kinetic energy. After the liner radius decreases several times with respect to its initial value, the plasma pressure inside the liner becomes larger than the magnetic pressure outside the liner, and the liner decelerates. At the point of maximum compression, it stops and then expands again under the action of the plasma pressure. The liner dynamics is considered in more detail in Sec. IV.

The liner is assumed to be made of some heavy material, and its velocity during all phases of the implosion is assumed to be much smaller than the plasma sound velocity. Therefore, plasma compression occurs in the adiabatic manner.

Of most interest is the dynamics of the system near the point of the maximum compression where the fusion flash can occur. For the plasma parameters that will be obtained in the subsequent analysis, the maximum plasma pressure is such that the compressibility of the liner material becomes important. To eliminate the adverse effect of the liner compressibility (which reduces possible compression rates), we consider only thin liners, whose thickness even in the final state is less than the plasma radius in this state. In such a case, a first rough assessment of the liner dynamics can be carried out under the approximation that the liner is just an infinitely thin massive surface; the liner compressibility for a thin liner becomes, obviously, unimportant. This model of a structureless liner will be used throughout this paper. The liner will be characterized by its linear mass density $\mu$ (mass per unit length in the $z$ direction). The finiteness of the liner thickness can still play some role near the point of a maximum compression (see Sec. VII).

We introduce the elongation of the FRC as the ratio of its length $L$ to its diameter $2 a$ :

$$
E=L / 2 a \text {. }
$$

We consider a self-similar two-dimensional compression that maintains a constant value of $E$. This means that the FRC should be compressed not only radially but also axially. A concrete way of achieving this mode of compression is to properly tailor the mass density of the liner along the axis ${ }^{13}$. If the central part is heavier than the ends, its compression lags behind, and the shape of the liner evolves, as shown in Fig. 2, maintaining an approximate constancy of $E$. Exact constancy of $E$ is not of principal importance. However, because current experiments
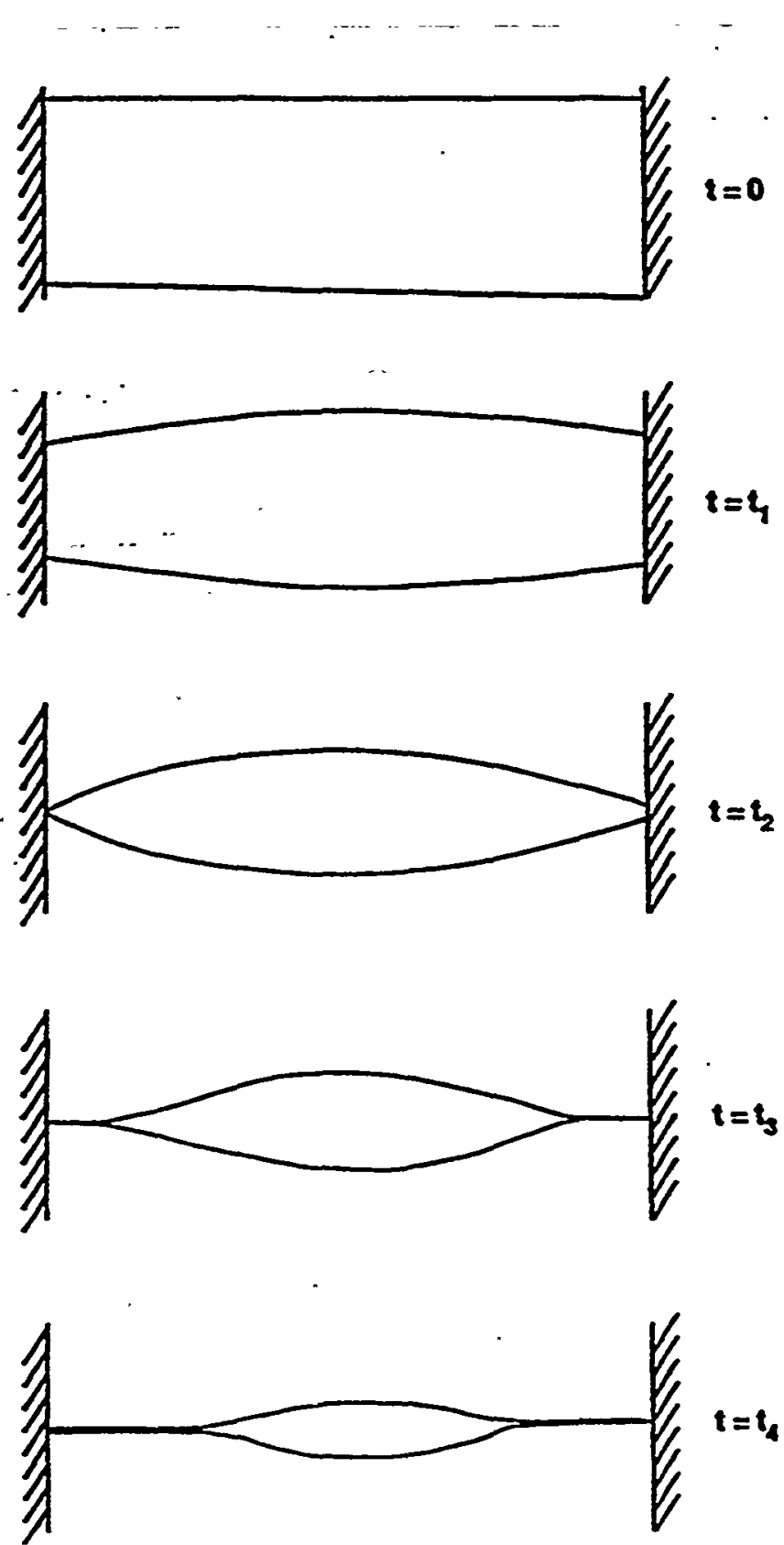

Fig. 2. By tailoring the mass density along the liner, one can achieve a two-dimensional compression as illustrated here. The ends of the liner have less mass density and implode more quickly to maintain the chosen elongation of the confined magnetic structure. The current can be terminated sometime between $t=t_{1}$ and $t=t_{2}$. 
with FRCs show the best performance for $E=3$ to 4, we believe that it would be reasonable to maintain $E$ relatively close to 3 to 4 . Another reason for choosing $E=3$ to 4 is that this value seems convenient from the viewpoint of the implosion physics (not too large to make the kink instabilities of great concem and not too small to make the electrode effects dominant). For spheromaks, elongations of -3 are probably too high because of internal tilting and twisting that occurs at such elongations. ${ }^{14}$ To make a spheromak stable with respect to these modes, smaller elongations, $E<2$, are preferable. ${ }^{\text {t4 }}$ For the elongations $E=1$ to 1.5 , the spherical liner of the type described in Ref. 1 may become preferable.

For $E=$ const, we can characterize the compression by a single parameter

$$
A=a_{0} / a \text {. }
$$

where $a_{0}$ and $a$ are the initial radius and the radius at any subsequent time, respectively, of the liner in the equatorial plane. We call $A$ the compression ratio.

As Fig. 2 shows, we imply that the end parts of the liner collapse on the axis relatively early in the discharge. They certainly trap a $z$ component of the magnetic field; i.e., near the axis, a kind of mix of the liner material and axial magnetic field is present. The liner material collapsed on the axis should gradually expand in the radial direction. But, because of the very high thermal capacity of heavy material, the expansion will occur at a relatively slow rate. In any case, the dynamics of the end sections of the liner after their collapse has little influence on what goes on in the central part, where the plasma object is nested.

Other possible candidates for configurations to be placed into the liner are the prolate spheromak (Fig. 3) and the Z-pinch configuration (Fig. 4). In this latter case, the azimuthal component of the magnetic field inside the liner does not necessarily serve for the plasma confinement (which can be provided by the material walls), and the Bennett relationship does not necessarily hold. In terms of the problem under consideration, the sphero-

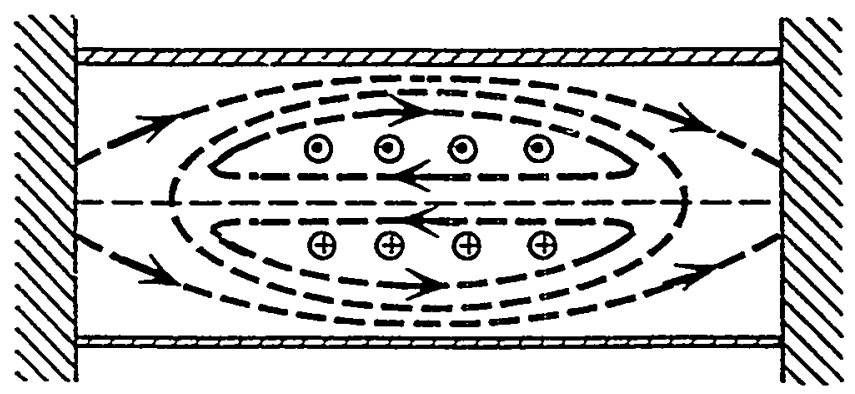

Fig. 3. An alternative scheme would use a spheromak inside the liner. Here the poioidal magnetic fields are indicated by dashed lines. and there are also toroidal magnetic fields as shown.

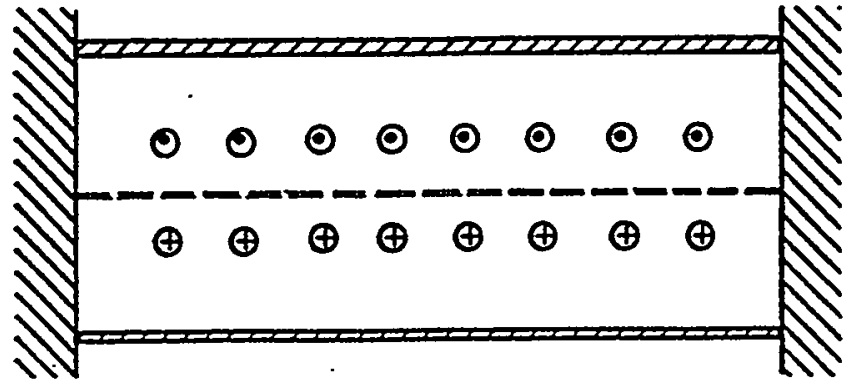

Fig. 4. Another alternative scheme would use a Z-pinch inside the liner. which would be simpler to produce but would obviously have worse confinement.

mak configuration does not differ much from the FRC. If the prolate spheromak can be magnetohydrodynamically (MHD) stable at $\beta>1$, it should be considered a good candidate for the adiabatic compression experiment. An obvious difference of the $Z$-pinch configuration from the other two is that in the Z-pinch geometry, the particle drift trajectories intersect the walls (more specifically, the end walls). Still, for low- $Q$ systems, this may not be too severe of a problem. As for the drift losses of the alpha particles in the $Z$-pinch, they occur within such a short time that one cannot count on any alpha-particle heating. This is of little concern for the low- $Q$ systems but may become important at $Q>5$. Discussion of the physics of the $Z$-pinch in the range of densities similar to that assumed in our paper can be found in Ref. 15.

The obvious advantage of the Z-pinch for filling a liner is that this configuration can be easily created inside the liner just by using a simple electrode discharge. The preparation of a miniature FRC or a spineromak will probably require some ingenuity. One possible approach could be the preparation of these objects outside the liner and translation of them into the liner through a hole in one of the electrodes. Existing experiments clearly show that translation of FRCs and spheromaks is possible. ${ }^{16,17}$ Then, the liner implosion should be organized in such a way that on the hole side of the discharge, the liner would collapse on the axis relatively early in time to prevent the ejection of the plasma back into the preparation chamber. The preparation of the FRC and spheromak inside the liner is also conceivable. In this case, the liner would have to have cuts in the axial direction to allow the $Z$ component of the magnetic field to penetrate into the liner. In case of a spheromak, one can in principle count on the helicity injection technique of the type described in Refs. 18 and 19. We will not discuss formation of the initial plasma configuration in any more detail in the present paper. From the viewpoint of the basic scaling laws discussed in Sec. III, all three are equivalent. All three will eventually evolve into the wali-confined plasma objects with $\beta \gg 1$. An option for improved performance would be to produce $\beta \gg 1$ in the initial plasma, which might 


\section{Drake et al. SUBMEGAJOULE LINER IMPLOSION OF A CLOSED FIELD LINE CONFIGURATION}

for example be accomplished by laser heating or stagnation of a rapidly translating plasma.

\section{SCALING LAWS FOR A THREE-DIMENSIONAL IMPLOSION}

We assume that from the very first moments of the plasma creation, the plasma conductivity is high enough that the magnetic field is frozen into the plasma. Later, we give some numerical examples supporting this assumption. For a perfectly conducting plasma, the magnetic field strength scales inversely to the square of linear dimensions:

$$
B=B_{0} A^{2} \text {, }
$$

and the magnetic pressure scales as $A^{4}$ :

$$
p_{M}=p_{M 0} A^{4} \text {. }
$$

Neglecting plasma heat losses (we show later that they are indeed not very important) and assuming that the plasma behaves as a monatomic ideal gas, we obtain the following scaling laws for plasma temperature $T$, plasma density $\rho$, and plasma pressure $p$ :

$$
\begin{aligned}
& T=T_{0} A^{2}, \\
& n=n_{0} A^{3},
\end{aligned}
$$

and

$$
p=p_{0} A^{5}
$$

(recall that the subscript 0 relates to the initial values of the corresponding quantities). From Eqs. (6) and (9), we see that the plasma beta scales as $A$ :

$$
\beta=\beta_{0} A \text {. }
$$

In other words, having plasma with initially comparable magnetic and gas-kinetic pressures, one very soon obtains a plasma where gas-kinetic pressure is dominant. This is an important advantage of the two-dimensional. compression.

If the compression is one-dimensional, the plasma pressure scales as $A^{10 / 3}$, while the $z$ component of the magnetic field scales as $A^{2}$, and the magnetic pressure in the FRC and spheromak soon becomes dominant. Therefore, in this latter case, the liner spends its energy mostly on the compression of the magnetic field. In addition, the geometric self-similarity breaks, and the plasma elongation becomes very large, increasing the probability of instabilities. This clearly shows the advantage of a twodimensional over a one-dimensional compression.

'This is not the case when the filling consists of a $Z$-pinch: The $\varphi$ component of the magnetic field scales then as $A$, and the magnetic pressure remains small.
Other important parameters characterizing the plasma behavior are the magnetization parameter $\omega_{B i} \tau_{i}$; the parameter $s=a / \rho_{i}$, which shows how many ion gyroradii can be accommodated within the plasma radius and is an indicator of plasma susceptibility to various drift-type instabilities (the larger $s$, the less susceptible); and plasma collisionality $a / \lambda_{i i}$. These parameters scale as

$$
\begin{aligned}
\omega_{B i} \tau_{i} & =\left(\omega_{B i} \tau_{i}\right)_{0} A^{2}, \\
s & =s_{0},
\end{aligned}
$$

and

$$
a / \lambda_{i i}=\left(a / \lambda_{i i}\right)_{0} A^{-2}
$$

\section{IMPLOSION DYHAMICS}

For elongations considerably exceeding the unity, the motion of the liner can be considered.as a quasi-radial one: It is possible to neglect the axial displacement of any point of the liner compared with its radial displacement. This does not contradict the two-dimensional nature of a plasma compression: Even though every point of the liner moves in the radial direction only, the shape of the liner changes to provide a two-dimensional compression (see Fig. 2). As the compression is very slow compared with the sound speed of the plasma, the pressure inside the imploding shell remains uniform.

We can write equations of motion for the equatorial cross section of the liner. As the mass per unit area of the liner is $\mu / 2 \pi a$, the equation of motion is

$$
(\mu / 2 \pi a) \ddot{a}=p-p^{(e x t)},
$$

where $p$ and $p^{(e x r)}$ are the plasma pressure inside the liner and the pressure of the azimuthal magnetic field outside the liner, respectively. Let us assume that the $Z$-pinch current is turned on at $t=0$ and is maintained constant at later times. In real life, the current will have a finite rise time, so that our estimates will give somewhat shorter implosion times than for a realistic waveform. Still, basic scaling laws remain the same, and our results can serve as a general guide.

In the case of a constant current, the external pressure scales as $A^{2}$ :

$$
p^{(e x t)}=p_{0}^{(e x t)} A^{2} .
$$

Recalling relationships (4) and (9), one can present Eq. (14) in the form

$$
(\mu / 2 \pi) d^{2} / d t^{2}(1 / A)=p_{0} A^{4}-p_{0}^{(e x t)} A .
$$

Equation (16) has the energy integral

$$
\left(\mu / 2 \pi A^{4}\right)\left(\dot{A}^{2} / 2\right)+\left[\left(p_{0} / 3\right) A^{3}-p_{0}^{(e x t)} \log A\right]=\text { const . }
$$


The first term represents the kinetic energy of the liner; the other two terms can be interpreted as the potential energy of the system. They represent a negative contribution from the compressing force and a positive contribution from the plasma internal energy. Because initially the liner is at rest and the compression ratio $A$ is just equal to 1, the right side of Eq. (17) can be rewritten as $p_{0} / 3$ :

$$
\left(\mu / 2 \pi A^{4}\right)\left(\dot{A}^{2} / 2\right)+\left[\left(p_{0} / 3\right) A^{3}-p_{0}^{(\text {ext })} \log A\right]=p_{0} / 3 .
$$

To attain a high compression ratio, one should have $p_{0}$ considerably smaller than $p_{0}^{(e x r)}$ :

$$
p_{0} \ll p_{0}^{(e x)} \text {. }
$$

An important result that readily follows from Eq. (18) is the relationship between the maximum compression ratio $A=A_{\max }$ and the initial driving pressure $p_{0}^{\text {(ext) }} ;$ to find it, one should just put $\dot{A}=0$. The result reads

$$
p_{0}^{(e x t)} / p_{0}=\left(A_{\max }^{3}-1\right) / 3 \log A_{\max } .
$$

This relationship is illustrated by Fig. 5. The other observation that can be made on the basis of Eqs. (18) and (19) is that at the dominant part of the compression process, when $A$ is already greater than, for example, 1.5 but still less by a factor of 1.5 than its final value determined by Eq. (20), the compression is governed by the equation

$$
\left(\mu / 2 \pi A^{4}\right)\left(\dot{A}^{2} / 2\right)=p_{0}^{(e x t)} \log A .
$$

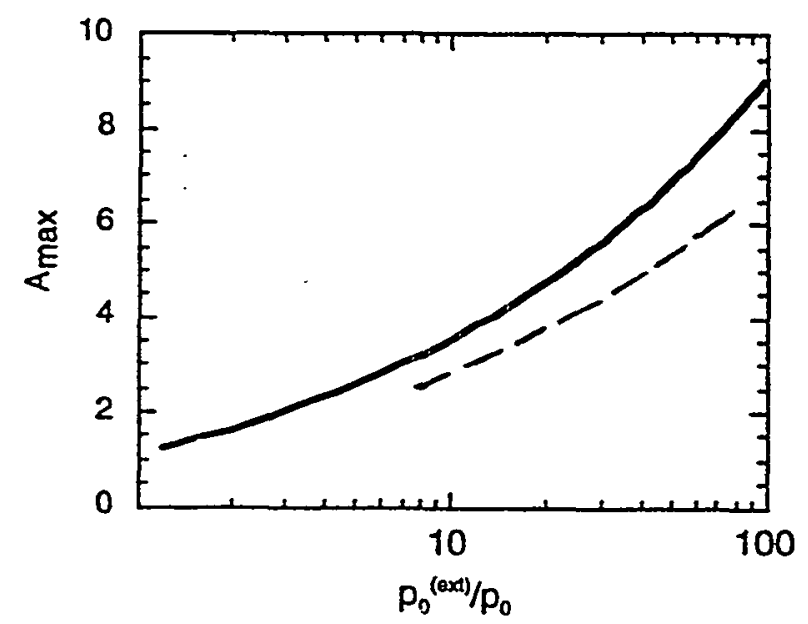

Fig. 5. The maximum obtainable compression depends (as shown by the solid line) on $p_{\delta}^{(e x)} / p_{0}$. which is the ratio of the initial external pressure on the liner to the initial internal plasma pressure. To obtain high compressions, which are required for gain, one must have this ratio quite large. The dashed line shows the same dependence for the case when the current is terminated at $A^{*}=2.7$.
If one defines the instantaneous compression time $\tau_{\text {comp }}$ as $\tau_{\text {comp }}=|A / \dot{A}|$, then one finds from Eq. (21) that

$$
\tau_{\text {comp }}=\left[\mu / 2 \pi p_{0}^{(e x t)} A^{2} \log A\right]^{1 / 2} \text {. }
$$

This expression is formally divergent near the initial point $(A=1)$; for $A<0.5$, a better measure is the total compression time, which will be introduced shortly. For the adiabatic approximation to remain valid during all the phases of the compression, $\tau_{\text {comp }}$ should be much shorter than the plasma cooling time. We return to the discussion of this issue in Sec. VI.

The total compression time $\tau_{\text {total }}$ (by which we mean the time from turning on the $Z$ current until the time of maximum compression) can be found by the integration of Eq. (18). For the final compression ratios exceeding 3 to 4, the following approximate result is valid:

$$
\tau_{\text {toral }}=\left[\pi \mu / 16 p_{0}^{(e x t)}\right]^{1 / 2} .
$$

According to Eqs. (9) and (20), the maximum plasma pressure is

$$
p_{\max }=p_{0}^{(e x t)}\left\{\left[p_{0}^{(e x x)} / p_{0}\right]\right\}^{2 / 3}\left\{\log \left[3 p_{0}^{(e x t)} / p_{0}\right]\right\}^{5 / 3} .
$$

Clearly, if condition (19) holds, the maximum pressure exceeds not only $p_{0}$ but also $p_{0}^{(e x)}$.

As can be seen from relationship (21), until a very late phase of the implosion, the extemal source converts its energy mostly into the kinetic energy of the liner. And, only very near the maximum compression point, the liner converts its energy into the plasma energy. A practical implication of this conclusion is that one can turn off the $Z$-pinch current relatively early in the implosion phase, for example, at $A=2$ to 3 , and still obtain a final plasma pressure not much different from estimate (23). A relatively early termination of the current in the liner may, in turn, be desirable to avoid a thermal explosion of the liner..$^{20}$ Although it may be possible to accelerate the liner even after this explosion occurs, it is probably more reliable to stay within the range of the driving magnetic fields that do not cause a thermal explosion (i.e., in our case, below the magnetic field of 1 to $1.5 \mathrm{MG}$; see Ref. 20).

Let us denote by $A^{*}$ the value of $A$ at which the $Z$-pinch current gets terminated. From the energy conservation law in the form of Eq. (21), we find that the kinetic energy of the liner at this point is equal to

$$
p_{0}^{(e x t)} \log A^{*} \text {. }
$$

Further compression occurs according to the conservation law. Eq. (17), with the third term in the left side omitted (as there is no external magnetic field any more). The right side can be determined from the matching conditions at the point $A=A^{*}$ (which, we assume, is far from the point of the maximum compression). In this way, one 
readily obtains the following expression for $A_{\max }$ in this more sophisticated version of the liner implosion:

$$
A_{\text {max }}=\left\{\left[p_{0}^{(e x)} / p_{0}\right] \log \left(\dot{A}^{* 3}\right)\right\}^{1 / 3} .
$$

We see that, indeed, even for relatively small values of $A^{*}$ (relatively early termination of the plasma current) the maximum compression ratio is not very different from the one determined by relationship (20).

One should not, of course, forget the problem of liner stability, especially at the deceleration phase near the stagnation point. In this respect, the system under consideration does not look much different from other systems employing imploding liners. In Ref. 5, a two-dimensional compression of the empty liner was studied. It was reported that for a liner manufactured accurately enough, there were no signs of gross instabilities, provided that the inaccuracies in the initial liner configuration were $<1 \%$. If the liner is "stuffed" with the plasma, of particular concern should be the Rayleigh-Taylor instability at the plasma-liner interface near the point of a maximum compression. Again, accurate enough manufacturing should be of some help in this respect.

\section{PARAMETERS OF A CONCEIVABLE EXPERIMENT}

In this section, we present a possible set of plasma parameters that would allow attainment of a breakeven condition at relatively low input energy. To avoid overcomplicating, we assume that no auxiliary heating sources are used to produce the initial plasma so that the initial beta value is close to 1 . The initial FRC will be produced on a relatively slow timescale, exceeding the Alfvern transit time. We presume that for these slow (few-microsecond range) magnetic fields, the upper limit will be relatively low:

$$
B_{0}<100 \mathrm{kG} \text {. }
$$

Condition (1) relates the initial temperature, density, and magnetic field in an obvious way:

$$
\beta_{0} B_{0}^{2} / 8 \pi=2 n_{0} T_{0} .
$$

Because the plasma temperatire $T_{\max }$ at the moment of maximum compression should be equal to $\sim 10 \mathrm{keV}$, the required maximum compression ratio is uniquely determined by the initial plasma temperature via reiationship (7):

$$
A_{\max }=\left(T_{\max } / T_{0}\right)^{1 / 2}
$$

Using as input parameters initial magnetic field and initial plasma temperature and substituting them into relationships ( 8 ) and (9), one can easily find the final plasma parameters:

$$
\begin{aligned}
& n_{\max }=\left(\beta_{0} B_{0}^{2} / 16 \pi T_{0}\right)\left(T_{\max } / T_{0}\right)^{3 / 2}, \\
& p_{\max }=\left(\beta_{0} B_{0}^{2} / 8 \pi\right)\left(T_{\max } / T_{0}\right)^{5 / 2} .
\end{aligned}
$$

To evaluate the plasma energy in the final state, we should make some assumptions regarding the size of the $Z$-pinch. We assume that its initial dimensions are $a_{0}=$ $1 \mathrm{~cm}$ and $L_{0}=6 \mathrm{~cm}$ (i.e., $E=3$ ). The supporting arguments for this choice of parameters will be presented in Sec. VII. As soon as the initial dimensions are chosen, we can evaluate the final plasma energy:

$$
W_{\max }=\pi a_{\min }^{2} L_{\min }\left(3 p_{\max } / 2\right)=\left(\frac{3}{8}\right) E \beta_{0} a_{0}^{3} B_{0}^{2}\left(T_{\max } / T_{0}\right)
$$

The physical quantities in Eqs. (29) and (30) (and throughout) are in Gaussian cgs units. Figure 6 depicts the plasma energy versus the initial plasma temperature for the aforementioned initial dimensions of the FRC and several values of the initial magnetic. field. As we see, it is quite conceivable to obtain fusion-grade plasmas with an energy content in the range of $100 \mathrm{~kJ}$. The required compression ratio is determined by Eq. (28). For the initial FRC temperatures of 100 to $400 \mathrm{eV}$ (which are quite common in the current experiments ${ }^{12}$ ), the required compression ratio is only 5 to 10 .

Although at present there is no experience in creating dense $\left(-10^{18} \mathrm{~cm}^{-3}\right)$ centimetre-sized FRCs, the basic parameters that determine their MHD behavior are in our case the same as in the existing experiments (operating with densities of $-10^{15} \mathrm{~cm}^{-3}$ and radii of $-10 \mathrm{~cm}$ ). Therefore, there is a reason to believe that the FRC with required parameters can be created. With regard to the

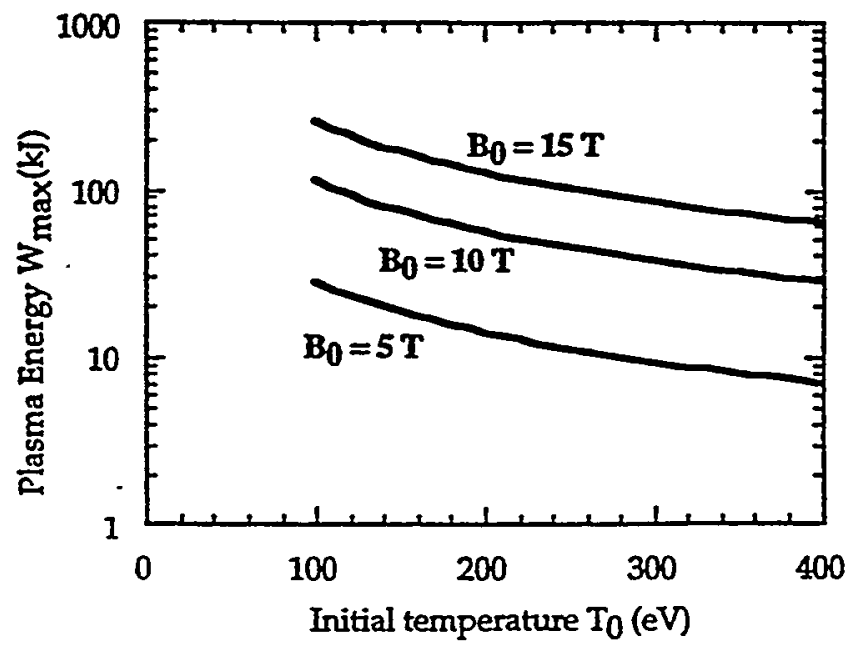

Fig. 6. The maximum energy stored in the plasma depends on the initial plasma iemperature for the three indicaled values of the initial magnetic field. as shown. The other parameters are $\beta_{0}=1, a_{0}=1 \mathrm{~cm}$, and $E=3$. Plasma energies.above $100 \mathrm{~kJ}$ appear fcasible. 


\section{Drake et al. SUBMEGAJOULE LINER IMPLOSION OF A CLOSED FIELD LINE CONFIGURATION}

spheromak configuration, the present experimental data cover only the domain of relatively low beta's: $\beta<0.1$ (Ref. 14). Little is known on whether the wall confinement would allow raising the beta up to $\sim 1$; further study of this issue is required.

For further numerical estimates, we use the set of parameters presented in Table 1. For this set of parameters, the gyroradius of fusion alpha particles in the final state is equal to $0.03 \mathrm{~cm}$ and is smaller by a factor of 3 than a plasma radius. Therefore. if the drift trajectories of the alpha particles are closed inside the plasma volume (as in the FRC and spheromak configurations), a considerable fraction of the alpha particles is confined and deposits its energy in the plasma. In the Z-pinch geometry, the alpha particles will drift away along the axis before depositing any substantial amount of their energy into the plasma.

In Table II, we present a possible set of the parameters of the liner and the value of the initial external current that are compatible with the plasma parameters listed in Table I. We show the liner parameters in the equatorial plane. We find the required external initial magnetic field $B_{0}^{(e x t)}$ from relationship (20), with $p_{0}$ and $A_{\max }$ corresponding to Table I. We choose the initial liner thickness in such a way that the final liner thickness is equal to the final plasma radius [more specifically, we choose parameter $\xi$ in Eq. (40) to be equal to 1]. This pushes our analysis to its applicability limit. However, the main parameters of the system are insensitive to $\xi$ (in particular, the plasma gain $Q$ scales as $\xi^{1 / 3}$ ), and therefore, the assumption $\xi=1$ should not have a strong effect on our conclusions regarding the system performance.

Liners with an initial radius-to-thickness ratio $a_{0} / \Delta_{0}$ in the range of a few tens have been successfully used in some implosion experiments. In the experiment of Ref. 5, a two-dimensional implosion of the liner with $a_{0} / \Delta_{0}=40$ to 100 was studied. A volume compression ratio of $\sim 1000$ (which corresponds to $A_{\max }$ of $\sim 10$ in our notations) was reported. In Ref. 1 , the ratio $a_{0} / \Delta_{0}$

TABLE I

Parameters of a Conceivable Experiment

\begin{tabular}{|l|c|c|}
\hline \multicolumn{1}{|c|}{ Parameter } & Initial State & Final State \\
\hline Plasma temperature $(\mathrm{keV})$ & 0.1 & 10 \\
Magnetic field $(\mathrm{T})$ & 10 & $10^{3}$ \\
Plasma beta & 1 & 10 \\
Plasma density $\left(\mathrm{cm}^{-3}\right)$ & $1.25 \times 10^{18}$ & $1.25 \times 10^{21}$ \\
Plasma radius $(\mathrm{cm})$ & 1 & 0.1 \\
Plasma elongation, $E$ & 3 & 3 \\
Plasma energy $(\mathrm{kJ})$ & 1 & 100 \\
Magnetized parameter, $\omega_{B t} \tau_{t}$ & 1 & 100 \\
Parameter, $s=a / \rho_{\prime}$ & 40 & 40 \\
Collisionality. $a / \lambda_{n}$ & 40 & 0.4 \\
\hline
\end{tabular}

TABLE II

Parameters of the Compressing System

\begin{tabular}{|c|c|}
\hline $\begin{array}{l}\text { Initial liner radius }(\mathrm{cm}) \\
\text { Final liner radius }(\mathrm{cm}) \\
\text { Initial liner thickness }(\mathrm{cm}) \\
\text { Final liner thickness }(\mathrm{cm}) \\
\text { Current through the liner (MA) }\end{array}$ & $\begin{array}{l}1 \\
0.1 \\
0.01 \\
0.1 \\
5\end{array}$ \\
\hline $\begin{array}{l}\text { Initial magnetic field at the outer } \\
\text { surface of the liner (T) } \\
\text { Liner density }\left(\mathrm{g} / \mathrm{cm}^{3}\right) \\
\left.\text { Total compression time, Eq. }\left(22^{\prime}\right) \text { ( } \mu \mathrm{s}\right) \\
\text { Liner dwell time [Eq. (39)] near the point } \\
\text { of maximum compression ( } \mu \mathrm{s})\end{array}$ & $\begin{array}{c}100 \\
20 \\
2 \\
0.15\end{array}$ \\
\hline
\end{tabular}

was up to 40 , with $A_{\max }$ also approaching 10 . So, the assumptions regarding the liner performance made in this paper are justified in view of the past experiments.

In our considerations, we have assumed that the $Z$ current that drives the liner does not penetrate through the liner into the compressed plasma. This is certainly true if the liner thickness exceeds the skin depth for the characteristic time of the order of $\tau_{\text {toral }}$. For the typical set of parameters listed in Tables I and II, $\tau_{\text {trtal }}$ exceeds the current penetration time by a factor of 2 to 3 , so that some of the driving current would switch to the plasma. However, until very late in the compression phase, the plasma conductivity is orders of magnitude smaller than the liner conductivity, and the fraction of the driving current switched to the plasma remains small.

If necessary, one can completely eliminate any penetration of the driving current into the plasma by using a two-layer liner. The outer layer can be made of aluminum and have a thickness exceeding the skin depth; the inner layer should be made of a heavy material. A possible example for the parametric domain covered by Tables I and II could be $0.3 \mathrm{~mm}$ of aluminum ( $\rho=2.7$ $\left.\mathrm{g} / \mathrm{cm}^{3}\right)$ and $0.1 \mathrm{~mm}$ of gold $\left(\rho=18 \mathrm{~g} / \mathrm{cm}^{3}\right)$. The outer shell is lighter than the inner one and will not considerably affect the implosion dynamics. It will prevent the driving current from penetrating into the heavy inner shell and into the plasma. The presence of this thick outer shell will also eliminate any problems of a premature thermal explosion of the inner part of the liner.

\section{ENERGY LOSSES FROM THE PLASMA}

In the system under consideration, plasma pressure exceeds the magnetic pressure, and the mechanical equilibrium of the plasma is provided by the presence of material walls. The magnetic field serves only to suppress the plasma thermal conductivity to the walls. What we have here is a classical case of so-called wall confinement (see, e.g., a survey talk by the first proponent of 
this concept, G. 1. Budker '). The confinement time in this case depends on whether the walls confine the magnetic flux or whether their conductivity is poor and the magnetic field penetrates into the walls. In this latter case, no magnetic cushion of a very high magnetic field can be formed near the wall. As one could expect from intuitive considerations and as the numerical and analytical studies ${ }^{21.22}$ show, the case of a poorly conducting wall is worse in terms of the plasma confinement. In our further estimates, we admit just this worst-case model and show that nonetheless the predicted thermal losses remain small.

Of course, even for a nonconducting wall, the magnetic field in the hot plasma interior remains frozen into the plasma, but in the colder regions in the vicinity of the wall where plasma density goes up to maintain the constancy of the plasma pressure and where one might expect a corresponding increase of the magnetic field, this increase in the case of poorly conducting walls is quite moderate. ${ }^{22}$ Despite the fact that plasma cooling in the near-wall regions is accompanied by plasma convection to the wall, the global confinement time still scales roughly as the plasma radius square (see a summary of the corresponding studies in Ref. 22). It turns out that for a plasma beta of -10 to 30 and a magnetization parameter of -30 to 300 (as the ones to be expected in the experiment under consideration; see Sec. V), the plasma confinement time for poorly conducting walls follows just the Bohm scaling. with effective thermal diffusivity $\chi_{\text {eff }}$ by $a$ factor of a few smaller than the Bohn thermal diflusivity:

$$
\chi_{\text {eff }}=(\alpha / 16)(c T / e B) \text {, }
$$

where $c x$ is -0.1 and $T$ and $B$ are the plasma temperature and magnetic field in the bulk of the plasma (not near the wall). The plasma cooling time can be evaluated in the standard way:

$$
r_{x}=a^{2} / 6 \chi_{\text {eff }},
$$

where the numerical coefficient in the denominator correspunds to cylindrical geometry.

We will evaluate the plasma cooling time for the reference case mentioned in Sec. V. One can easily find that for $\alpha=0.1, \tau_{x}$ in this case is $2 \times 10^{-6}$ s. so that the $n \tau_{x}$ product is as high as $3 \times 10^{15} \mathrm{~cm}^{-3} \cdot \mathrm{s}$ and would correspond to a quite high plasma enhancement factor $Q$ of the order of 30 . Therefore, we see that the thermal conductivity to the walls does not significantly affect the performance of the system even for the worst-case model (poorly conducting walls).

In the system under consideration in the immediate vicinity of the magnetic axis, there exists a region that is directly connected to the end walls along the magnetic field lines or (as in the case of a Z-pinch stuffing) is just field free (remember that there is no magnetic field on the Z-pinch axis). One can expect high electron thermal losses along this needlelike structure. Accordingly, it should remain cold and dense during the whole compression process. The overall effect of this paraxial structure on the plasma confinement is not quite clear yet, but we can obtain an upper estimate of the heat losses to this cold needle by just assuming that in its place, a cold material cylinder has been introduced. Then, just because the surface of this central cylinder is quite small compared with the total inner surface of the liner, the addition of this cylinder would not significantly increase the overall losses.

It is quite clear that under the action of the heat flow from the plasma and Joule dissipation in the skin layer at the inner side of the liner, this inner surface will be evaporated and ionized. As both the jon gyroradius and mean free path of these relatively cold ions will be two to three orders of magnitude smaller than the plasma radius, they will not directly penetrate into the plasma. In addition, it turns out that in a high-beta plasma, the impurities get repelled from the hot region under the action of the thermal force and plasma convection toward the walls. ${ }^{23}$

In Sec. V, we showed that the instantaneous compression time scales as $\tau_{\text {comp }}-1 / A$ [see Eq. (22)]. For our assumptions on the adiabatic nature of the compression to be valid during the entire compression process, the implosion time should be uniformly shorter than the time of the heat loss to the walls and the time of the radiative losses:

$$
\tau_{\text {comp }} / \tau_{x} \ll 1, \tau_{\text {camp }} / \tau_{\text {rad }} \ll 1 .
$$

The thermal conduction time $\tau_{x}$ scales as $\sim 1 / A^{2}$, so that the ratio $\tau_{\text {comp }} / \tau_{x}$ scales as $A$. The time of the radiative (bremsstrahlung) cooling by definition is

$$
\tau_{\text {rad }}=3 n T / q_{\text {rad }} \text {, }
$$

where $q_{\text {rad }}$ is the power radiated per unit plasma volume and $q_{\text {rad }}=C n^{2} T^{1 / 2}$. The radiation cooling time also scales as $1 / A^{2}$, and the ratio $\tau_{\text {comp }} / \tau_{\text {rad }}$ Scales as $A$. Therefore, it is sufficient to show that inequalities (33) hold near the point of maximum compression. We will make the corresponding estimates in Sec. VII. Here, we just note that for the parameters of Table I. the thermal loss times at the point of the maximum compression are

$$
\tau_{x} \sim 2 \times 10^{-6} \mathrm{~s}, \tau_{\text {rad }} \sim 2 \times 10^{-6} \mathrm{~s} .
$$

We should also check that the magnetic field is frozen into the hot plasma core of the FRC; otherwise, the entire aforementioned model of wall confinement breaks down. The magnetic diffusivity $D_{M}$ can be determined from the relationship

$$
D_{M}=\left(c^{2} / \omega_{p e}^{2}\right) \nu_{e i},
$$

where $\omega_{p e}$ and $\nu_{e i}$ are the electron Langmuir frequency and electron-ion collision frequency, respectively. If $\nu_{p i}$ is determined just by Coulomb collisions, $D_{M}$ is extremely small. Of some concern might be a situation where 
$\nu_{e i}$ would be determined by some kind of anomalous scattering of the electrons. But, as long as $\nu_{e i}$ is less than a fraction of the electron gyrofrequency $\omega_{B e}$, the magnetic diffusivity can be ignored. Indeed, for $\nu_{e i}=\omega_{B e}$, the ratio of the magnetic diffusivity, Eq. (36), to the Bohm thermal diffusivity, Eq. (31), is

$$
D_{M} / \chi_{\text {eff }}=64 / \alpha \beta \text {. }
$$

As is shown in Sec. VII, the time of heat loss, Eq. (32), is at least a factor of 20 longer than the compression time even at the most dangerous point of maximum compression. At this point, $\beta=10$ and $D_{M}$ exceeds $\chi_{\text {eff }}$ by a factor of -30 . Therefore, even in the most dangerous part of the compression process, the magnetic diffusion time is longer than the compression time, even for a very pessimistic assumption that $\nu_{e i}=\omega_{B e}$ [in fact, because the current density in the system under consideration is much smaller than $e n v_{\pi_{i}} \equiv e n(2 T / M)^{1 / 3}$, it is likely that electron collisions will be just classical].

\section{DYNAMICS OF THE SYSTEM NEAR THE STAGNATION POINT}

As we have shown in Sec. VI, the thermal conductivity does not considerably affect the plasma performance for the standard set of.plasma parameters. In this section, we show that the attainable $Q$ value is, in fact, limited by the expansion of the liner under the action of plasma pressure. Near the point of the maximum compression. Eq. (14) can be rewritten in a simplified form:

$$
d^{2}\left(a-a_{\min }\right) / d t^{2}=\left(2 \pi a_{\min } / \mu\right) p_{\max } .
$$

We have neglected the external magnetic field pressure (because it is small compared with the plasma pressure inside the liner at $A=A_{\text {max }}$ ). We have also neglected the fusion energy release, so that plasma pressure is an even function of time with respect to the maximum compression point. The latter assumption is justified by the fact that, as we will see shortly, the attainable $Q$ values are not high enough to make the alpha-particle heating important. Finally, we restricted ourselves to phenomena occurring at $a^{\prime} s$ not very much different from $a_{\min }$ and replaced plasma pressure by a constant equal to $p_{\text {mar }}$.

A considerable reduction of the fusion power release with respect to its maximum value at the point of maximum convergence occurs when the plasma volume increases, roughly speaking, by a factor of 2 , or in other words, the plasma radius increases by $30 \%$ with respect to its minimum value. We will use the time during which the plasma radius stays within the limits $a_{\min }<a<$ $a_{m m}(1+\epsilon)$, with $\epsilon \sim 0.3$, as the time of a fusion energy release $\tau_{\text {fur }}$. From Eq. (38), one readily finds that

$$
\tau_{f u s}=2\left(\mu \epsilon / \pi p_{\max }\right)^{1 / 2} \text {. }
$$

The time $\tau_{\text {fus }}$ increases with the linear mass density of the liner $\mu$. The latter is limited from above by the requirement that the liner thickness $\Delta$ in the stagnation point not exceed some fraction $\xi$ of the plasma radius; otherwise, the compressibility of the liner would become important (see Sec. II). Therefore, we use the following estimate for $\mu$ :

$$
\mu=2 \pi \Delta \rho_{L} a_{\min }=2 \pi \xi \rho_{L} a_{\min }^{2},
$$

where $\rho_{L}$ is the density of the liner material. This relationship can be used for rough estimates until $\xi \sim 1$ (the dependence of plasma enhancement $Q$ on $\xi$ is weak). We use the following standard scaling law for the plasma enhancement $Q$ :

$$
Q=K n \tau_{\text {fus }},
$$

where $K$ is the Lawson's constant, $K=10^{-34} \mathrm{~cm}^{3} / \mathrm{s}$. Combining relationships (39), (40), and (41) and using expression (30) for the plasma energy content in the final state, one can obtain the following relationship between $Q$ and $W_{\text {max }}$ :

$Q=K\left(\epsilon \xi \beta_{0}^{1 / 3} \rho_{L} / 3^{2 / 3} \pi E^{2 / 3} T_{0} T_{\max }\right)^{1 / 2}\left(W_{\max } B_{0}\right)^{1 / 3}$.

For the set of parameters shown in Table $I$ and for $\epsilon=$ $0.3, \xi=1$, and $\rho_{L}=20 \mathrm{~g} / \mathrm{cm}^{3}$, we find that $Q \sim 1.5$. If all the parameters but $W_{\max }$ and $B_{0}$ are kept constant, the scaling for $Q$ acquires the form

$$
Q=0.15\left[W_{\max }(\mathrm{kJ}) B_{0}(\mathrm{~T})\right]^{1 / 3} .
$$

Now we return to a discussion of the possible role of thermal losses. As has been already mentioned, for the set of parameters of Table $1, Q-1$. Then, according to Eq. (41), $\tau_{\text {fus }}=1.5 \times 10^{-7} \mathrm{~s}$; in other words, the dwell time is, roughly speaking, 20 times less than both the thermal conductivity and radiative cooling time [see expression (35)]. Therefore, the thermal losses from the plasma can, indeed, be neglected throughout the whole compression process.

It is interesting to note that the slowing-down time of the alpha particles is much shorter than the time of the fusion flash: For the set of parameters presented in Table I, the time within which the alpha particle loses one-half of its initial energy is only $25 \mathrm{~ns}$, while the liner dwell time is -150 ns.

\section{REACTOR ISSUES AND POSSIBLE NONFUSION APPLICATIONS}

One discouraging feature of the conventional approaches to fusion energy is that they do not appear to lend themselves to a small reactor for developmental purposes. This is in contrast to the normal evolution of a new technology, which typically proceeds to a full-scale conmercial plant via a set of graduated steps. Therefore, the prospects for the near-term realization of fusion. and 
perhaps the ultimate commercial reactor, would be improved if it could be introduced through a compact, cheap system such as the wall-confined system of this paper.

There have been several examples in the literature of repetitive, pulsed-power reactor concepts based on the Z-pinch (see, for example, Refs. 24 through 28). We might expect that reactors based on our concept would share some of the critical technology issues of these Z-pinch reactors. However, one significant advantage relative to the dense Z-pinch is the relaxation of the very high rate of current rise and, therefore, a greater flexibility in the design of the primary circuit with regard to inductance limitations. Probably the three most important critical issues for a viable reactor based on our liner-driven FRC are (a) the requirements for a system to supply electrical input energy to the liners at high repetition rates for long periods, (b) electrodes capable of continuous operation over long periods and sustaining acceptably low damage rates from multiple discharges, and (c) a first-wall assembly capable of absorbing repeated bombardment from the burn (fusion products and liner debris).

Because of the high thermal loads on the electrode tips, some form of liquid-cooled or all-liquid-metal electrodes will probably be required. Candidate configurations have been discussed by Robson ${ }^{26}$ and Bolton et al. ${ }^{28}$ Robson, for example, visualizes a low-inductance system comprising two orthogonal, nonintersecting liquid lithium electrodes in which the fusion assembly (in this case a fiber pinch) is formed across the shortest distance between them. The liquid lithium electrodes are linear jets projected from one insulated terminal and caught in another at the same electrical potential. With a distance of $2 \mathrm{~m}$ between terminals and a jet velocity of $25 \mathrm{~m} / \mathrm{s}$, the jets drop by only $-3 \mathrm{~cm}$. In this way, any hot spots formed on the electrodes by the fusion burn will be carried away from the source region and should not affect the electrical properties of the system, provided the pulse repetition rate is not too high.

Energy of $1 \mathrm{MJ}$ or less can be delivered to the system as the kinetic energy of fast projectiles. This would allow the spatial separation of the power supply system and be the point where the fusion microexplosion occurs. Thereby, one would eliminate any problems of the neutron damage and neutron activation to the power supply system.

One possible way of realizing this latter concept is illustrated in Fig. 7. We assume that a flat projectile 1 (of the type obtained in the Osher et al. experiment ${ }^{29}$ ) compresses a magnetic flux of a pre-existing magnetic field into a small volume 2 . The load would be a liner driven either as a $Z$-pinch or as a theta pinch. The initial bias magnetic field can be produced by permanent magnets. This scheme implies that the whole assembly 2 , with thie liner already in place, would be dropped into the reaction chamber before each shot. The cost of these disposable elements should be small (see estimates of the cost of the shot later in this section). The initial magnetic field can be also generated by a current transported to the load in the form of a high-power electron beam (Fig. 8).

Vacuum requirements in the case of liquid lithium electrodes are probably determined by the electrical insulation of the electrodes, which might suggest a chamber pressure in the range of $\sim 10^{-5}$ to $10^{-4}$ Torr or below. If electrode jets were employed, then the exposed lithium surfaces would act as getter pumps for residual deuterium-tritium (D-T) gas. Because of continuous bombardment from reaction products and liner debris, the use of a conventional solid first wall is probably precluded here. However, because of the geometrical simplicity of our concept, wetted wall concepts could be employed analogous to those used in inertial confinement fusion (ICF) reactors. ${ }^{30}$ Complete bulk blankets of exposed liquid with first walls sustained by rotating vortices have been proposed for simple fusion geometries like these. 28.31 However, because of vapor pressure limits in our chamber, temperatures of exposed lithium surfaces (e.g., electrode jets and wall coatings) should probably be kept below $\sim 350^{\circ} \mathrm{C}$, i.e., a vapor pressure of $\sim 10^{-5}$ Torr. Therefore, because of low thermodynamic efficiency, this may preclude the use of a fully exposed liquid lithium blanket system but may permit the use of other candidate liquids with lower vapor pressures such as Flibe."

Relationship (42) shows that the plasma enhancement factor $Q$ depends relatively weakly on the parameters of the system. For example, to increase $Q$ from 1.5 to 3 , one would have to increase $W_{\max }$ to $0.8 \mathrm{MJ}$. Therefore, in its present form, the concept that we are considering would work as the basis of an energy-producing facility only at the level of the input energies exceeding 10 MJ. Potentially, $Q$ can be increased somewhat by lengthening the liner dwell time near the point of maximum compression. One possible way of achieving this objective is an ablation of some amount of the liner material near the maximum compression point and use of the resulting reactive force to balance the plasma pressure near the turning point. The ablation could be produced by a pulsed laser. Simple estimates show that an increase of $Q$ by a factor of 2 is possible in this way, but more detailed assessment would be desirable.

Another seemingly obvious way of increasing $Q$ is just increasing the liner thickness or, in other words, increasing parameter $\xi$. However, as has already been mentioned under the expected standard conditions listed in Table I, the maximum plasma pressure will be already so high that the compressibility of the liner material will become important. Even at $\xi \sim 1$, the compressibility effects might already play some role, but because of a relatively weak dependence of $Q$ on $\xi$, they would not considerably affect estimates (42) and (43). Evaluation of the potentialities of the thicker liners would require a more detailed study.

We can write the net power generated by an electricity-producing plant based on our liner-driven capsule scheme as 


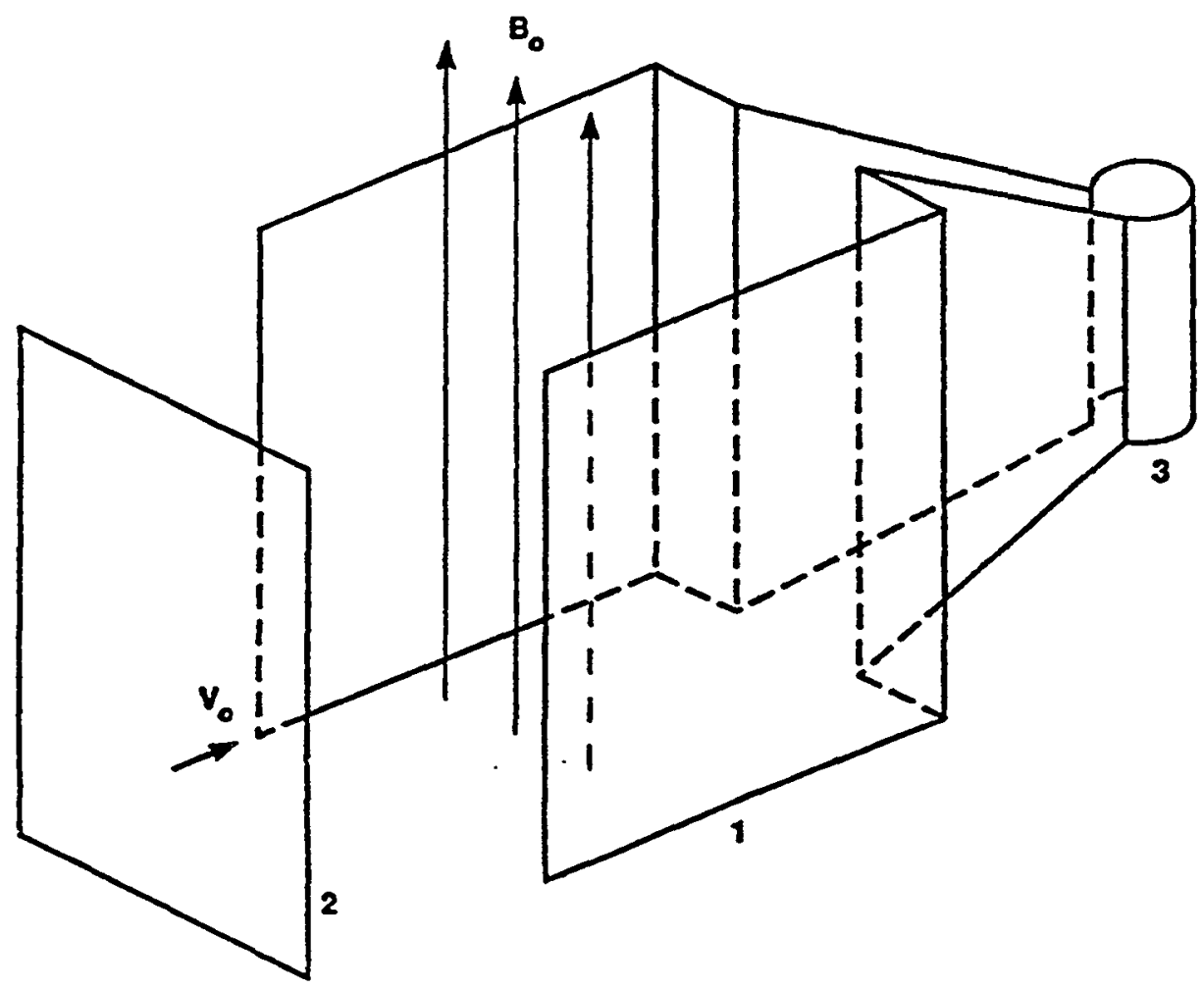

Fig. 7. A possible concept of a detached energy source: 1 is a volume with conducting walls enclosing an initial magnetic field $B_{0}-1 \mathrm{~T}: 2$ is a fast flat projectile that, after closing the circuit, compresses the magnetic field into a small volume 3 where the liner (not shown) can be nested. In the version shown in this figure, the driving magnetic field has a theta-pinch configuration. The initial volume occupied by the magnetic field is $-10^{3} \mathrm{~cm}^{3}$. For the characteristic speed of the fast projectile of $\sim 10^{6} \mathrm{~cm} / \mathrm{s}$, the rise time of the magnetic field will be of the order of a few microseconds. By using a proper circuitry, one can apply this scheme also for driving the initial current in the magnetic configuration.

$$
P_{\text {e.net }}=r Q W\left(1-f_{\text {cux }}^{\cdot}\right)\left[0.8 M \eta_{t h}-1 /\left(Q \eta_{i}\right)\right] .
$$

where

$$
\begin{aligned}
r= & \text { repetition rate } \\
W= & \text { input drive energy (i.e., } Q W \text { is the fusion en- } \\
& \text { ergy released per shot) } \\
M= & \text { blanket energy multiplication } \\
\eta_{i}= & \text { efficiency of conversion from wall plug en- } \\
& \text { ergy to input energy } \\
\eta_{t h}= & \text { thermal cycle efficiency } \\
f_{\text {cux }}= & \text { fraction of gross electrical power required to } \\
& \text { supply the plant auxiliaries other than the en- } \\
& \text { ergy input to the fusion core itself. }
\end{aligned}
$$

Equation (44) assumes that no useful electrical power is generated from either input power $(r W)$ or the fusion alpha-particle power $(r Q W / 5)$.

Taking typical values of, for example, $M \sim 1.3, \eta_{t h} \sim$ $0.35, \eta_{i} \sim 0.6$, and $f_{a u x} \sim 0.05$, Fig. 9 shows the required

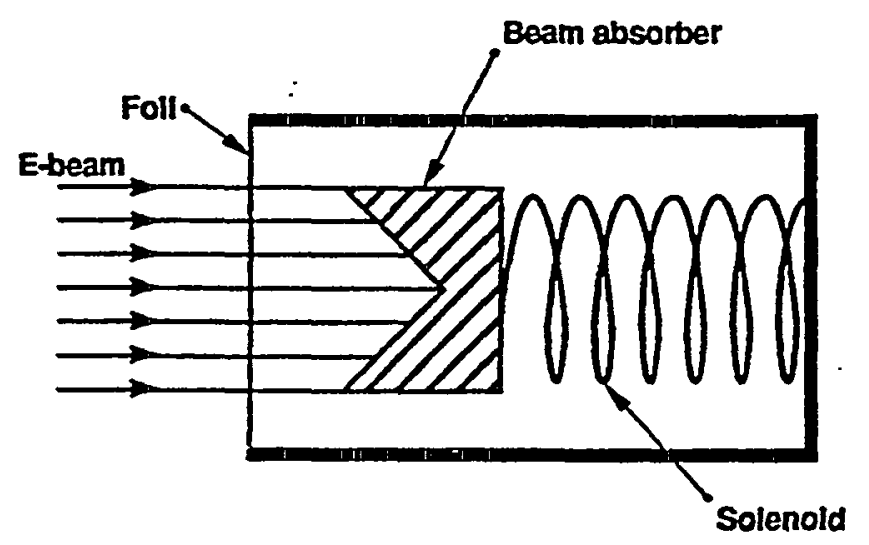

Fig. 8. Another version of the detached energy source: An electron beam with a current of a few kiloamperes and energy of $-0.5 \mathrm{MeV}$ is transported through a low-pressure gas to the beam absorber, which is separated by a thin foil from the external gas; the beam drives a current in a solenoid that can serve as an inductive energy store for initiating the plasma current in our configuration; this scheme can be used in combination with that shown in Fig. 7. 
Drake e c al. SUBMEGAJOULE LINER IMPLOSION OF A CLOSED FIELD LINE CONFIGURATION

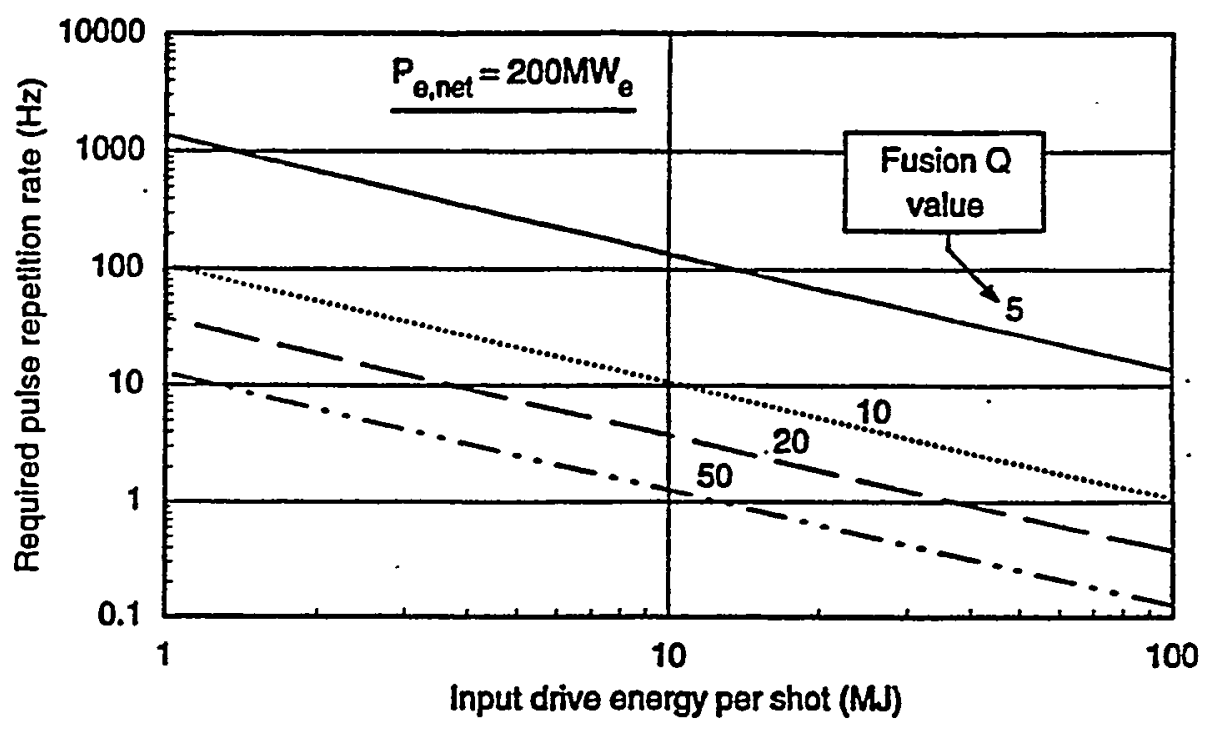

Fig. 9. Energy parametrics for a 200-MW(electric) pulsed, liner-driven FRC reactor. The required pulse repetition frequency is plotted as a function of the input drive energy for various values of the fusion gain $Q$. Assumptions on plant performance are supplied in the text.

repetition rate $r$ to sustain a $200-\mathrm{MW}$ (electric) net reactor as a function of the input energy $W$ to the liner-driven capsule for various values of the fusion $Q$ value. Note that no net electric power is possible for such a reactor for $Q$ values less than $\sim 5$. Great benefits are seen by increasing the $Q$ to the range of 20 to 30 . In particular, for $Q=20$, an input energy of $12 \mathrm{MJ}$ would require a pulse repetition rate of only $-3 \mathrm{~Hz}$, surely feasible for a repetitive mechanical target system such as this. Under such conditions, the energy loading on a liquid first wall would be $-300 \mathrm{MJ}$. This is very similar to that of an ICF reactor, although there would be a greater momentum transfer from the liner mass relative to the hohlraum mass of an indirect-drive ICF capsule. Certainly, the $3-\mathrm{Hz}$ repetition rate is sufficiently low to allow for adequate chamber clearing between shots. Therefore, further work on the proposed scheme should concentrate on ways to considerably increase $Q$.

To be economically competitive with the projected competition for the early twenty-first century (e.g., advanced fission and natural gas), our power plant should exhibit a cost of electricity (COE) at the bussbar of no more than $\sim 5 \phi / \mathrm{kW} \cdot \mathrm{h}$ for a $100-\mathrm{MW}$ (electric) plant and perhaps twice this for a 200-MW(electric) plant. ${ }^{32.33}$ This, of course, constraints the upper limit of the capital cost of the reactor plant per kilowatt of fusion power generated.

Note also that the requirement of COE of $\sim 5$ to $10 \notin 1$ $\mathrm{kW} \cdot \mathrm{h}$ constrains the upper limit of the cost of each linerdriven capsule together with its $D-T$ fuel load. Assuming that the fuel costs contribute no more than, for example. $10 \%$ of the COE requires that each capsule in our 200$\mathrm{MW}$ (electric) plant be fabricated for approximately $\leq \$ 0.6 / r$, where $r$ is the pulse repetition rate. For our earlier example of $Q=15 . W=20 \mathrm{MJ}$, and $r \sim 3 \mathrm{~Hz}$, each target should, therefore, cost no more than $\sim 20$ to fabricate and recycle. Thus, fuel costs may also constrain the pulse repetition rate to lower values.

Finally, with the promise that our liner-driven FRC concept can be realized in a small, compact system, we note that even if it should prove to exhibit only low to moderate $Q$ 's, there are several other potential applications of this technology in addition to commercial electric power production. These include

1. application to commercial fission waste streams for the burning and transmuting of long-lived fission products and actinides ${ }^{b}$

2. application to the breeding of fissile fuel (in either the ${ }^{238} \mathrm{U}-{ }^{239} \mathrm{Pu}$ or ${ }^{232} \mathrm{Th}-{ }^{233} \mathrm{U}$ cycles) to support the future generation of advanced burner fission plants

3. medical isotope production

4. a small fusion source for the development of materials for future D-T fusion reactors of other configurations

5. At the level of $Q \sim 1$, our system can serve as a pulsed source of $X$ rays in the energy range of $! 0$ to $50 \mathrm{keV}$ and neutrons $\left(\sim 5 \times 10^{16} \mathrm{n} /\right.$ pulse $)$.

bIn an associated study, ${ }^{24}$ we suggest that a fusion-based waste transmutation facility should be competitive with the proposed accelerator-based, neutron spallation source in terms of neutrons per unit wall plug power if $\eta_{i} Q-0.2$. where $\eta_{i}$ is the conversion efficiency from wall plug power to input power. Taking $\eta_{i}$ as $\sim 0.5$ requires only $Q \geq 0.4$ for viability. 
In conclusion, we again stress the key point of our concept, i.e., the real potential for a significantly cheaper and less complex development path to realize an operating, proof-of-principle fusion reactor.

\section{PHYSICS ISSUES FOR THE FURTHER CONSIDERATION}

We have presented a very rough overall physical picture of the phenomena that should accompany compression of the closed field line configuration. In this section, we mention more subtle phenomena that, nevertheless, can have some effect on plasma performance.

Under the action of the heat flow from the plasma, some amount of the liner material will be evaporated and ionized. By itself, this would not cause any serious problems because this material would be kept near the liner surface by the plasma pressure. According to Sec. VI, heavy ions would be confined near the wall under the action of thermal force. However, the situation may deteriorate if some fast convective instabilities develop, giving rise to the intermixing of the liner material and the hydrogen plasma. Near the liner turning point, where the effective gravity force is directed against the density gradient, the instability could be of a Rayleigh-Taylor type. Öne can expect that this instability will be slower than the gross Rayleigh-Taylor instability of the liner itself because of the stabilizing role of dissipative processes, which will play a stronger role in small-scale instability. As was already mentioned in Sec. IV, according to Ref. 5, the gross liner instabilities did not cause considerable deformation of the liner if the manufacturing accuracy was $>1 \%$. Therefore, we can expect that the liner will be essentially axisymmetric. The presence of a heavy axisymmetric underlying surface would be one more stabilizing factor in the development of an instability of a thin film of a dense plasma. Considerable improvement of the liner stability could be attained also by setting the liner into initially slow rotation that would then accelerate because of rotational momentum conservation. This method of stabilization is discussed in some detail in Ref. 3.

Ablation of some amount of material from the outer surface of the liner near the turning point (see Sec. VIII) could reduce the liner acceleration at this most critical point and thereby additionally suppress all the gravitydriven instabilities. The dynamics of the ablation process is another issue that would require more attention.

Of some concern also should be the instability of the magnetic configuration driven by the regions of the unfavorable curvature of the magnetic field lines. One can expect that the presence of heavy material boundaries will stabilize the global modes. The local modes could be stabilized by the finite Larmor radius effects. Of particular interest would be the stability of the $Z$-pinch stuffing: This configuration is the one that can be formed by the sim- plest means (compared with the other two) and is, therefore, the most attractive for possible initial experiments with $Q \sim 1$ (for higher $Q$ 's, the issue of alpha- particle losses can come to the forefront).

When the end sections of the liner collapse on the axis, the ejection of thin cumulative jets along the axis may occur. The following should be analyzed in more detail: Can this phenomenon be avoided, perhaps by rotation, and if not, what could be the consequences of the presence of a thin needle of the liner material near the axis?

\section{SUMMARY AND DISCUSSION}

We have shown that adiabatic compression of a closed field line configuration (in particular, FRC) with an initial plasma density of $-10^{18} \mathrm{~cm}^{-3}$, a $100-\mathrm{eV}$ temperature, and an initial volume of a few cubic centimetres could produce a fusion microexplosion with a plasma gain of $Q=1$ to 2 at an input energy level of $\sim 100 \mathrm{~kJ}$. The initial magnetic field should be in the range of $10 \mathrm{~T}$.

The compression is supposed to be done by a thinwall liner driven by a pulsed- $Z$ current. Both the current amplitude ( $5 \mathrm{MA}$ ) and pulse length (a fraction of a microsecond) are well within the reach of present-day technologies.

By proper tailoring of the thickness of the liner along the axis, one can provide the conditions for twodimensional compression. In this two-dimensional compression mode, the plasma pressure becomes higher than the magnetic pressure inside the liner, and all the liner energy gets spent on the plasma heating (not on the compression of the magnetic field). Therefore, a relatively inexpensive experiment would allow the study of the behavior of high-beta fusion plasma at and somewhat beyond the breakeven point. The configurations that can be studied are the FRC, spheromak, and Z-pinch.

In the present paper, we considered only one of several possible techniques of compressing the compact plasma formation: a liner driven by the axial current. In principle, other options aiso exist, in particular, a liner driven by the axial magnetic field (theta-pinch geometry). The use of two liners is also conceivable, with the inner liner containing the plasma and the outer liner collapsing on the inner one near the maximum compression point, thus providing better conditions for the inertial confinement.

Probably, the plasma $Q$ can be increased by a factor of 2 by using the thicker liners and/or by ablating some amount of the liner material near the turning point. A further increase of $Q$ would require more ingenious improvement of the whole concept.

In the low- $Q$ version (with $Q$ even less than 1 ), the system discussed in this paper could serve as a pulsed source of $X$ rays in the range of 10 to $50 \mathrm{keV}$ and of $14 \mathrm{MeV}$ neutrons. 
Drake ct al. SUBMEGAJOULE LINER IMPLOSION OF A CLOSED FIELD LINE CONFIGURATION

\section{ACKNOWLEDGMENT}

This work was performed in part under the auspices of the U.S. Department of Energy by Lawrence Livermore National Laboratory under contract W-7405-Eng-48.

\section{BEFERENCES}

I. J. H. DEGNAN et al., Phys. Reu. Lelt., 74.98 (1995).

2. A. I. BARCILON et al.. in Proc. 5th Conf. Plasma Physics and Conirolled Nuclear Fusion Research. Tokyo. Japan, November II-15. 1974, Vol. 2. p. 567, International Atomic Energy Agency (1975).

3. D. L. BOOK et al.. in Proc. 6th Conf. Plasma Physics and Controlled Nuclear Fusion Research. Berchtesgaden. Germany. October 6-13, 1976. Vol. 3. p. 507. International Atomic Energy Agency (1977).

4. A.R. SHERWOOD, E. L. CANTRELL. I. HENINS. H.W. HOIDA, T. R. JARBOE, R. C. MALONE, and J. MARSHALL. in Proc. 8th lnt. Conf. Plasma Physics and Controlled Nuclear Fusion Research, Brussels, Belgium. July 1-10, 1980. Vol. 2, p. 607. International Atomic Energy Agency (1981).

5. S. G. ALIKHANOV et al., in Proc. 6th Conf. Plasma Physic.s and Controlled Nuclear Fusion Research. Berchtesgaden. Germany. October 6-13. 1976. Vol. 3. p. 517. International Atomic Energy Agency (1977).

6. F. L. RIBE and A. R. SHERWOOD, in Fusion, Vol. 1. p. 59. E. TELLER. Ed.. Academic Press. New York (1981).

7. H. U. RAHMAN, F. J. WESSEL, and N. ROSTOKER, Phis. Rev: Letl., 74. 714 (1995).

8. R. C. KIRKPATRICK, "Magnetized Target Fusion-An Overview of the Concept," LA-UR-94-4354, Los Alamos National Laboratory (1994).

9. I. R. LINDEMUTH and R. C. KIRKPATRICK, Nucl. Fusion, 23. 263 (1983).

10. V. K. CHERNYSHEV et al.. presented at Institute of Electrical and Electronics Engineers Int. Conf. Plasma Science, Santa Fe, New Mexico, June 6-8, 1994.

II. G. I. BUDKER, in Proc. 6th European Conf. Controlled Fusion and Plasma Physics, Moscow, USSR, July 30-August 4. 1973, Vo!. 2. p. 136.

12. A. L. HOFFMAN. "Reactor Prospects and Present Status of Field-Reversed Configurations." Trans. Fusion Technol.. 27. 91 (Apr. 1995).

13. E. P. VELIKHOV. Private Communication (1971).

14. T. R. JARBOI: Plasma P'hys. Comtrolled Fusion, 36. 945 (1994).

15. C. W: HARTMAN. D. Y. CHENG. G. E. COOPER. J. L. bDDI.EMAN. and R. H. MUNGER. in Proc. 7th Comf. Plasma P'hissos and combrolled Nuclear Fusion Rescarch. innsbruck.
Austria, August 23-31, 1978, Vol. 3, p. 653, International Atomic Energy Agency (1979).

16. J. H. HAMMER, C. W. HARTMAN, J. L. EDDLEMAN, and H. S. MCLEAN, Phys. Rev. Lett.. 61. 2843 (1988).

17. S. OKADA et al.. presented at 15th International Atomic Energy Agency Conf. Plasma Physics and Controlled Nuclear Fusion Research, Seville, Spain, 1994.

18. T.H.JENSENandM.S. CHU, Phys. Fluids, 27,2881 (1984).

19. J. B. TAYLOR and M. F. TURNER. Nucl. Fusion, 29, 219 (1989).

20. H. KNOEPFEL. Pulsed High Magnetic Fields, North Holland Publishing Company, Amsterdam (1970).

21. P. Z. CHEBOTAEV, D. D. RYUTOV, M. D. SPEKTOR, and G. E. VEKSHTEIN, in Proc. 6th European Conf. Controlled Fusion and Plasma Physics, Moscow, USSR, July 30August 4. 1973, Vol. 2. p. 414.

22. G. E. VEKSHTEIN, in Reviews of Plasma Physics, Vol. 15. p. I. B. B. KADOMTSEV, Ed.. Consultants Bureau (1990).

23. G. E. VEKSHTEIN, D. D. RYUTOV, and P. Z. CHEBOTAEV, Sov. J. Plasma Phys., 1, 220 (1975).

24. C. W. HARTMAN, G. CARLSON. M. HOFFMAN, R. WERNER, and D. Y. CHENG, Nucl. Fusion, 17, 909 (1977).

25. R. L. HAGENSON. A.S. TSAI. R. A. KRAKOWSKI. and R. W. MOSES, Nucl. Fusion, 21.1351 (1981).

26. A. E. ROBSON. in Proc. Ist Int. Conf. Dense Z-Pinches for Fusion, Alexandria, Virginia, March 29-30, 1984. J. SETHIAN and K. GERBER. Eds.

27. A. E. ROBSON, in Proc. 2nd Int. Conf. Dense Z-Pinches for Fusion. Laguna Beach. Califomia, April 26-28, 1989, N. PEREIRA et al., Eds., American Institute of Physics (1989).

28. H. R. BOLTON et al., Fusion Eng. Des., 10. 9 (1989).

29. J. E. OSHER, G. BARNES, H. H. CHAU, R. S. LEE, C. LEE. R. SPEER, and R. C. WEINGART, IEEE Trans. Plasma Sci., 17, 392 (1989).

30. R. W. MOIR. "Improvements to the HYLIFE-II Inertial Fusion Power Plant Design," Fusion Technol., 26, 1169 (1994).

31. R.W.MOIR, "Liquid First Walls forMagnetic Fusion Energy Configurations," Nucl. Fusion (submitted for publication).

32. J. D. GALAMBOS, L. J. PERKINS, S. W. HANEY, and J. MANDREKAS, Nucl. Fusion, 35, 551 (1995).

33. R.A. KRAKOWSKI.C. G. BATHKE, R.L. MILLER, and K. A. W'ERLEY, "Lessons Learned from the Tokamak Advanced Reactor Innovation and Evaluation Study (ARIES)," Fusion Technol., 26, 1111 (1994).

34. L. J. PERKINS and C. W. HARTMAN. "The Application of a High-Puwer-Density, Fusion Neutron Suurce Based on the Continuous Flow Pinch to Plutonium Disposition, Fission Waste Transmutation and Driven. Sub-Critical Fission." Fusion Terhnol. (submitted for publication). 
R. Paul Drake (PhD, The Johns Hopkins University, 1979) is the former director of the Plasma Physics Research Institute at Lawrence Livermore National Laboratory (LLNL). He is currently on staff at the College of Engineering at the University of Michigan. His research interests include laser/plasma interactions, plasma hydrodynamics, and fusion.

James H. Hammer [PhD, University of California, Berkeley (UC Berkeley), 1979] is a physicist in the Physics and Space Technology Directorate at LLNL. His research interests include high-density magnetized plasmas such as $Z$-pinches and compact toroids, laser-driven hohlraums, the fast ignitor concept, and other alternative approaches to fusion.

Charles W. Hartman (PhD. University of California, Berkeley, 1960) is a research physicist at LLNL and a lecturer at UC Berkeley. In addition, he is one of the founders of the Energy and Resources Group at UC Berkeley. He is well known for his work on pinches, stellarators, levitrons, and many other fusion concepts.

L. John Perkins.(PhD, University of Birmingham, United Kingdom, 1978) is a physicist within the Magnetic.Fusion Energy Division at LLNL. His research interests include plasma engineering, conceptual fusion reactor design, and innovative approaches to fusion energy.

Dmitri D. Ryutov (PhD, Kurchatov Institute, Moscow, Russia, 1966) is a physicist at LLNL. His research interests include plasma physics and its applications, astrophysics, and environmental aspects of energy production. 
UCRL-JC-133716

PREPRINT

\section{A Problem of Stand-off Energy Sources for MTF}

\section{D.D. Ryutov}

This paper was prepared for submittal to

Z-Pinches for Energy

Albuquerque, NM

April 27-28, 1999

April 19, 1999

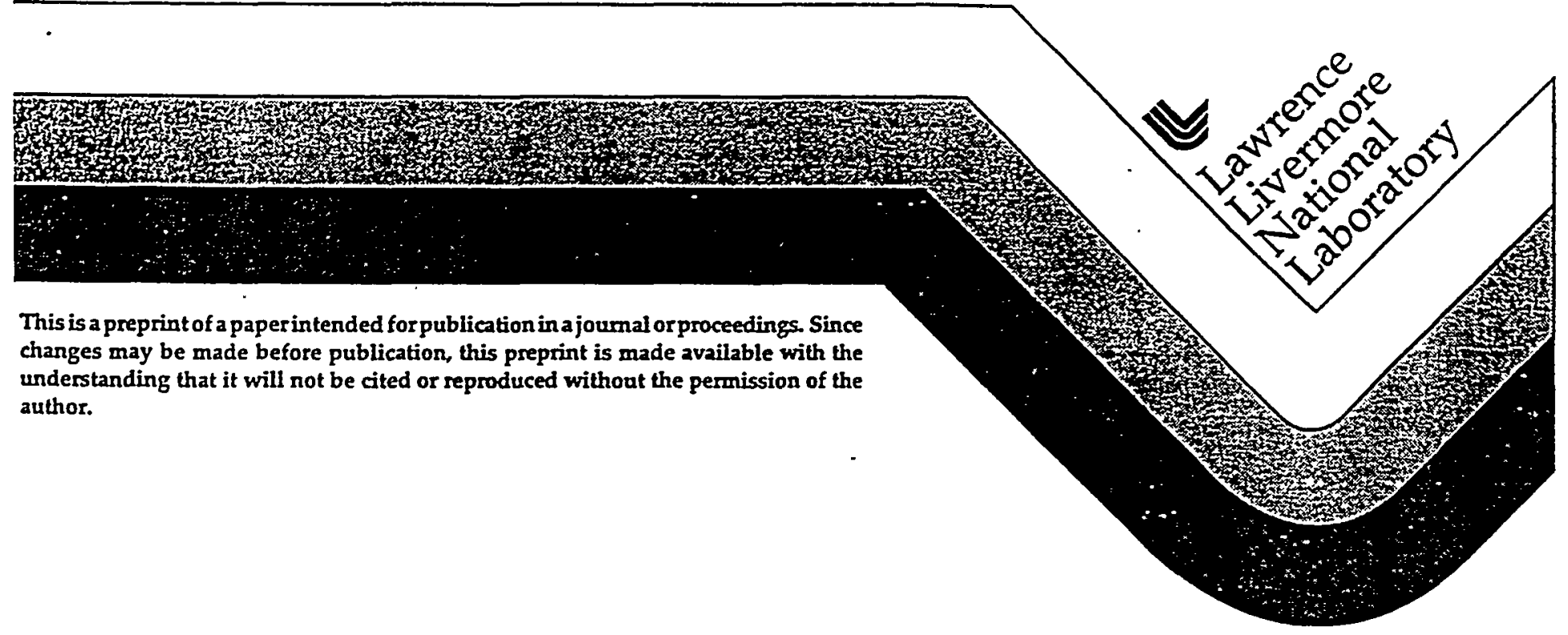




\section{DISCLAMMER}

This document was prepared as an account of work sponsored by an agency of the United States Government. Neither the United States Govemment nor the University of California nor any of their employees, makes any warranty, express or implied, or assumes any legal liability or responsibility for the accuracy, completeness, or usefulness of any information, apparatus, product, or process disclosed, or represents that its use would not infringe privately owned rights. Reference herein to any specific commercial product, process, or service by trade name, trademark, manufacturer, or otherwise, does not necessarily constitute or imply its endorsement, recommendation, or favoring by the United States Government or the University of Califormia. The views and opinions of authors expressed herein do not necessarily state or reflect those of the United States Government or the University of Califomia, and shall not be used for advertising or product endorsement purposes. 


\title{
A problem of stand-off energy sources for MTF
}

\author{
D.D. Ryutov \\ Lawrence Livermore National Laboratory, Livermore, CA 94551
}

Fusion devices based on the adiabatic (or shock) compression of the plasma by electromagnetically driven liner need specific energy sources capable of delivering a high current $(-10 \mathrm{MA})$ in the pulses $0.1-1$ microsecond long. In the present experimental facilities, the plasma load is situated very close to the pulsepower energy source. In the future fusion devices, one would have to place a plasma load at a considerable distance from the energy source (to avoid strong neutron and thermo-mechanical damage to the source). Several versions of the stand-off energy sources are considered. All are based on the idea of an "assembly" an object where the plasma load is nested and which contains all necessary circuitry that allows conversion of the energy delivered to the assembly into the magnetic energy. Such "assemblies" will be dropped (or insered) into the reaction chamber at a desired rate and energized by a stand-off energy source. Four specific concepts have been mentioned.

A concept of Magnetized Target Fusion (MTF) is very broad, encompassing fusion systems with the yields from many gigajoules per pulse $[1,2]$ to a few megajoules per pulse [3]. The repetition rate may also vary in a broad range, from $\sim 0.1 \mathrm{~s}^{-1}$ (for high-yield systems) to $\sim 20 \mathrm{~s}^{-1}$ (for low-yield systems). A common problem in all this variety of systems is a need in protecting the primary energy source from a neutron and thermomechanical damage associated with fusion energy release. The means to reach this goal may differ considerably depending on the yield and the repetition rate. We will briefly discuss here possible solutions for low-yield, high rep-rate systems. It should be noted that the problem of stand-off energy'sources is still in its infancy, and there are no detailed analyses available. The aim of this paper is merely to show that, at least at the level of basic physical principles, stand-off energy sources are feasible.

To be more specific, we discuss a version of MTF based on the use of a fieldreversed configuration (FRC), although the use of other magnetic configurations (spheromak, diffuse $Z$ pinch, and others) is also conceivable [3]. A possible way of solving the problem of a stand-off energy source has been delineated in Ref. [3], where it was suggested that the fusion reactor would work in the following way: the disposable assemblies (with the size of $30-50 \mathrm{~cm}$ ) would be dropped into reaction chamber (whose walls would be protected by liquid Li or LiPb flow, very much like in ICF reactors, Ref. [4]), and the energy required to drive the implosion would be delivered from the distance of tens of meters (see below). It was assumed that the assembly would contain the following elements: i) the system for pre-forming the FRC (or other configuration to be adiabatically compressed); ii) the liner, iii) the on-board circuitry required to energize various subsystems in a required sequence (formation of pre-plasma, translation it into the liner, liner implosion). 
To get some insight into the issue of what power supply systems may be needed, we consider creation of an FRC with the density $n \sim 10^{18} \mathrm{~cm}^{-3}$, the temperature $T \sim 100 \mathrm{eV}$, in a magnetic field of $B \sim 100 \mathrm{kG}$. This set of parameters corresponds to

$$
\beta \equiv \frac{16 \pi n T}{B^{2}} \sim 1
$$

The radius of the FRC can be $a \sim 1 \mathrm{~cm}$, and the length $L-4-6 \mathrm{~cm}$. Such an object could then be adiabatically compressed by an imploding liner (see Ref. [3] for a more detailed discussion and further references).

Magnetic coils of a radius $\sim 1.5 a$ would be used for creating the bias magnetic field and for the field reversal. The bias coil can have a relatively long pulse, up to a hundred microseconds. The field-reversal coil should be turned on within a time of order of several axial Alfven transit times [5],

$$
\tau=\alpha L / \mathrm{v}_{A},
$$

with $\alpha$ being of the order of 2 . For the aforementioned set of parameters, and for a deuterium plasma, one has $\tau \sim 1 \mu \mathrm{s}$. This estimate sets the time-scale for the controlled changes of the magnetic field.

The total energy content in the initial plasma will be $\sim 1 \mathrm{~kJ}$, and the magnetic energy will be several times higher, $\sim 3 \mathrm{~kJ}$ (because the magnetic field occupies a larger volume). For $\tau \sim 1 \mu$ s, the power level involved into the process of field reversal will be $\sim 3 \mathrm{GW}$. The current in the coil,

$$
I \sim \frac{c B L}{4 \pi},
$$

should be $\sim 1.5 \cdot 10^{15} \mathrm{CGS} \sim 0.5 \cdot \mathrm{MA}$ (for $B \sim 100 \mathrm{kG}$ and $L=6 \mathrm{~cm}$ ). The required loop voltage will be of the order of $7.5 \mathrm{kV}$. All these parameters are not very demanding.

At the temperature of $100 \mathrm{eV}$, the plasma will be fully ionized, and its radiative losses will be [6]:

$$
P_{r a d}(W)=1.7 \cdot 10^{-32} n^{2}\left(\mathrm{~cm}^{-3}\right) T^{1 / 2}(\mathrm{eV}) \cdot \pi a^{2}(\mathrm{~cm}) L(\mathrm{~cm})
$$

For the parameters given above, this power will be only $2.5 \mathrm{MW}$, much less than the total power delivered to the plasma during the reconnection event, $1 \mathrm{~kJ} / 1 \mu \mathrm{s} \sim 1 \mathrm{GW}$. This means that radiative losses from a pure plasma are negligibly small. For radiative losses to become considerable, the plasma should become very dirty, with the amount of heavy impurities (of the type of iron) in the range of $1 \%$. 
The FRC with the aforementioned parameters will have a ratio of a plasma radius to a characteristic ion gyro-radius of $-30-50$, much higher than in the existing experiments and very close to the values of this parameter expected for an FRC-based fusion reactor [3]. The pre-formed FRC will be translated into an imploding liner of the type described in Ref. 3 and then adiabatically compressed. We conceive of a scenario where the on-axis hole through which the FRC will be injected will be closed early in the implosion, thereby trapping the FRC inside the liner. This can be achieved by using a liner whose linear density (mass per unit length) on the injection end is smaller than over the rest of its length (Cf. Ref. [7])

The compression should be 3-dimensional, because in 3D implosions the energy is delivered predominantly to the plasma, not to the embedded magnetic field [3]. The feasibility of quasi-spherical implosions has been demonstrated in the experiments by Degnan et al. [8]. In geometrically self-similar 3D implosions, the plasma temperature scales as

$$
T=T_{0} C^{2},
$$

where $C$ is a linear convergence (the ratio of the initial dimension to the instantaneous dimension). If one starts with the plasma with the temperature $T_{0}=100 \mathrm{eV}$, the fusion-grade plasma needs reaching $C \sim 7-10$. Note that, in the aforementioned experiments by Degnan et al, the maximum linear convergence was close to 7 . According to the analysis carried out in Ref. [3], the life-time of the hot dense state is determined by the liner expansion under the action of the plasma pressure. For the liners with a mass of a few grams, one can obtain the fusion gain $Q \sim 10$. The energy delivered to the liner should be in the range of a few $\mathrm{MJ}$, with the characteristic time-scale of $1 \mu \mathrm{s}$ [3].

There are several ways of delivering the energy to the assembly dropped into the reaction chamber. The one is to use an "inverse diode" system [3], where the assembly would be energized by a 1-MeV electron beam, penetrating into the assembly through the entrance foil, being absorbed by a cathode, and generating a voltage between the foil and the cathode. With an appropriate circuitry (including, possibly, a pulse transformer) installed in the assembly, this energy source could be used to drive some fast circuits. The second approach employs generating supra-thermal electrons by illuminating a kind of a thermoionic diode attached to the "assembly" by intense light of a low-quality $\mathrm{CO}_{2}$ laser, and using these fast electrons to drive a current in the primary magnetic storage [9]. A third way is based on the use of fast flyers accelerated either electromagnetically (Ref. 10) or explosively (Ref. 11). These flyers could then be used to compress the conducting flux conserver that encloses some seed magnetic field (which could be generated, in particular, 
by the inverse diode system). The kinetic energy of the flyer would be converted into the magnetic energy and the latter would drive a circuit of the imploding liner. The flyers with velocities of order of $10^{7} \mathrm{~cm} / \mathrm{s}$ have been obtained in electromagnetic accelerators, with the flyer energy $\sim 100 \mathrm{~kJ}$ [10]. Explosively driven cumulative jets with velocities up to $9 \cdot 10^{6}$ $\mathrm{cm} / \mathrm{s}$ were also obtained [11]. Extrapolation to a few megajoules looks feasible, especially with explosively driven flyers. With a size of the flux conserver $\sim 10 \mathrm{~cm}$, one finds that a characteristic rise-time of the current generated by this magneto-compressive generator is $1 \mu \mathrm{s}$, matching the natural time-scale of the problem. The magnetic energy in a magnetocompressive generator increases in the inverse proportion to the cross-sectional area. If the energy delivered to the liner has to be $\sim 10 \mathrm{MJ}$, and the cross-sectional area is squeezed by a factor of 20 , the energy content in the bias magnetic field has to be $0.5 \mathrm{MJ}$. This can be attained by creating a bias field of $20 \mathrm{~T}$ in a flux conserver of initial volume $\sim 3 \ell$.

The fusion energy release inside the assembly will lead to its evaporation; the gas thus formed will be mixed with the LiPb gas formed because of evaporation of the protective liquid wall of the explosion chamber. To avoid the need in chemical separation of the mixture thus formed, it would be desirable to make the target of the same material as the liquid protective layer [12]. Both LiPb eutectic and a pure $\mathrm{Li}$ can be used as materials for the assemblies if cooled down to below minus $20 \mathrm{C}$. Some small amounts of other materials may still be needed in the assembly (to provide electrical insulation).

The practicality of this approach will depend not only on resolving a number of technical issues (which are quite challenging) but also on the possibility of mass-production of the assemblies (which would have to be delivered to the reaction chamber at a rate up to ten assemblies per second), and keeping their cost at the level of a few tens of cents per assembly. If the difficulties will prove insurmountable, one may consider systems with an increased yield $(\sim 200 \mathrm{MJ})$ and reduced rep rate $(\sim 1-2 \mathrm{~Hz})$. In this latter case it may become feasible to use direct mechanical connections with the external power supply, in the style discussed some time ago [13]. What we would like to emphasize is that the whole system can be made of the $\mathrm{LiPb}$ (with some minimum amount of insulating materials). To reduce the weight and improve mechanical properties, one could consider using a porous $\mathrm{LiPb}$ (or even a pure $\mathrm{Li}$ ) at a temperature in the range of minus $20 \mathrm{C}$. The density of this material, obviously, depends on its porosity and can be varied in a broad range. This circumstance allows one to tailor the density distribution around the point of the energy release in such a way as to produce significant hydrodynamic lensing [14], and direct the ejected material away from the most vulnerable elements of the reaction chamber. 
To summarize the present status of the problem: Solutions that would allow to deliver the properly conditioned energy to the liner situated at a distance of $\sim 10 \mathrm{~m}$ from the energy source, are feasible in principle. There is almost no doubts that one or even several of the aforementioned techniques can be realized in single-shot experiments. Main difficulties with the applications to a commercial generation of fusion energy are related to the feasibility of mass production of disposable elements at a low cost. It is desirable to direct some resources to the analysis of this problem.

Work performed under the auspices of the U.S. Department of Energy by Lawrence Livermore National Laboratory under Contract W-7405-ENG-48.

\section{References}

[1] P. Turchi. Proc. 3rd Int. Conf. on Megagauss Magn. Field Generation and Related Topics. Moscow, Nauka Publ. House, 1983, p. 184.

[2] I.R. Lindemuth, C.A. Eckdahl et al. Proc. 16 Intern. Conf. on Plasma Phys. and Controlled Nucl. Fus. Res., (Montreal, Canada, October 1996), International Atomic Energy Agency, Vienna, 1997, v.2, p.723.

[3] R.P. Drake, J. Hammer, C. Hartman, J. Perkins, and D.D. Ryutov. Fusion Technology, 30. 310 (1996).

[4] R. Moir. Phys. Plasmas, 2, 2447 (1995).

[5] M. Tuszhewski. Nucl. Fusion, 28, 2033 (1988).

[6] J.D. Huba. NRI Plasma Formulary, NRL/PU/6790-94-265, 1994.

17] S.G. Alikhanov, S.G., V.P. Bakhtin, et al. In Proc. 6th Conf. on Plasma Phys. and Contr. Nucl. Fusion Res., (Berchtesgaden Germany, October 1976), Vol. 3, p. 517, International Atomic Energy Agency, Vienna, 1977.

[8] J.H. Degnan et al. Phys. Rev. Lett., 74, 98 (1995).

[9] D. Ryutov. "Stand-off energy sources for pulsed fusion devices." Innovative Conf. Concepts Workshop, PPPL, April 4-6 1998, UCRL-JC-129697 Abs.

[10] J.E. Osher, G. Barnes, H.H. Chau, R.S. Lee, C. Lee, R. Speer, and R.C. Weingart. IEEE Trans. Plasma Sci., 17, 392 (1989)

[11] M.A. Lavrent'ev and B.V. Shabat. "Hydrodynamical Problems and their Mathematical Models," Moscow, Nauka Publishing House, 1973 (in Russian)

[12] B.G. Logan and J.H. Hammer. Private communication, 1998

[13] R.W. Moses, R.A. Krakowski, R.L. Miller. "A Conceptual Design of the Fast-Liner Reactor (FLR) for Fusion Power." LANL Report LA-7686-MS, February 1979 (http://ib-www.lanl.gov/la-pubs/00209074.pdf)

[14]G.B. Whitham. "Linear and Nonlinear Waves." NY, Wiley, 1974. 
Technical Information Department - Lawrence Livermore National Laboratury Unlversily of Californin - Livermore, Californin 94551

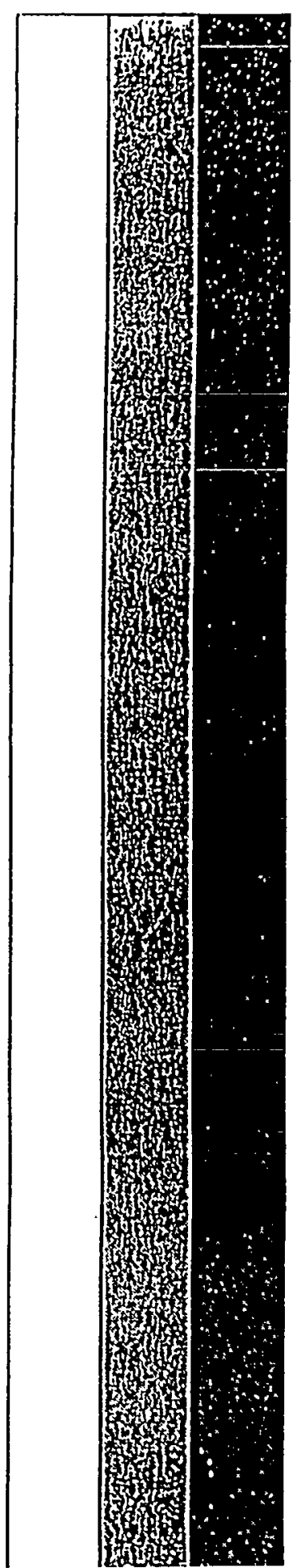




\section{INVERSE ION DIODES FOR DRIVING Z-PINCH IFE}

G. Cooperstein, P.F. Ottinger, and D. Mosher

Pulsed Power Physics Branch

Plasma Physics Division

Naval Research Laboratory

Washington, DC

Presented to

Z-Pinch IFE Workshop

April 27-28, 1999

\section{Outline}

- Brief history

- Conceptual overview

- Inverse electron beam diodes

- Inverse ion diodes

- Point design

- Ion source options

- Ion transport options

- Inverse diode options

- Issues 


\section{Brief History}

- SNL electron beam fusion research with wire initiated z-discharge

NRL transport (EBFA) - early 70 s

- Rudakov proposed capturing transported electron beams with an inverse electron beam diode and driving a z-pinch - mid 70s

- massive disposable transport channels

- Cornell applied-B ion diode research - early $70 \mathrm{~s}$

- NRL pinch-reflex ion diode research with protons- mid 705 to early 805 - focusing, z-discharge transport and final focusing

- SNL LIICF program - mid 70s to mid 90s

- applied-B diodes with heavier ions

- NRL, Cornell and other collaborators

- Recent NRL ion transport research

- low-mass z-discharge, wire-guided, ballistic, and self-pinch transport

- all of above part of SNL's LIICF or LBNL's HIF program

- Cooperstein suggested ion beam power convergence and inverse ion diodes for PRS loads at DNASNL Full Threat Simulator meeting in 10/92

- Slutz suggested ion beam driven z-pinches for IFE in $2 / 97$ memo

- Melhorn reiterated suggestion at BEAMS'98 (6/98)

\section{Conceptual Overview}

- Produce protons with ten or more self-magnetically insulated ion diodes with pulsed plasma anodes

- also consider applied-B or MAP ion diodes or

- higher- $Z$ ions from two-stage applied-B ion diode with stripping

- Transport ions to large inverse ion diode using ballistic transport

- also consider self-pinch transport or

- transport with laser-initiated Z-discharge channels or

- low-mass wall-confined z-discharge channels and/or

- bunching by factor of 2 or 3

- Capture ion beams in inverse barrel ion diode with electrons inhibited through self-magnetic insulation, and drive two annular z-pinches surrounding pellet in hejplraum

- also consider applied-B barrel ion diode with precharged capacitors 


\section{Advantages}

NRL

- Builds on over 20 years of Light Ion Inertial Confinement Fusion (LIICF) production, focusing and transport research

- Inverse ion diode z-pinch concept has > 10X larger target than LIICF

- relieves divergence constraint

- simplifies ion transport

- allows use of protons and simpler, self-magnetically insulated ion diodes both at source and at target

- Ion beam cool relative to electron beam and can be recaptured with higher efficiency

- Ion bunching could provide current multiplication during transport

- Charge- and current-neutral ion transport may provide easier path to energy convergence than self-magnetically insulated transmission lines (MITLS)

Simple 1-D Child Langmuir Inverse Electron Diode Illustrates Concept (no ions or self-magnetic field effects are included)

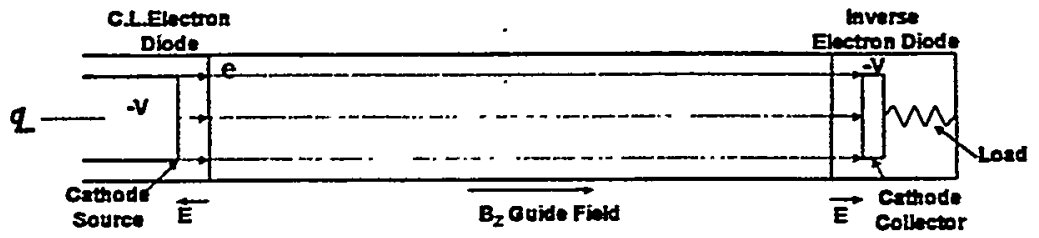

- Electrons accelerate to voltage

- Electrons decelerate from $V$ to zero along electric field and $V$ against electric field from cathode to anode soft-land on cathode collector

- $Z_{\text {diode }}=V / I$$$
\text { - } Z_{\text {lozd }}=V I I
$$

- Depressed collector schemes for Gyrotrons are example of successful application of this technique with cool laminar electron beams

- High temperature electron beam has low recapture efficiency

- "heat" cannot be recaptured

- also, losses to ions could be large 


Self-Magnetically Insulated Inverse Ion Diode Concept is
Complicated by 2-D Effects and Non-Intuitive Electron Orbits $N R L$

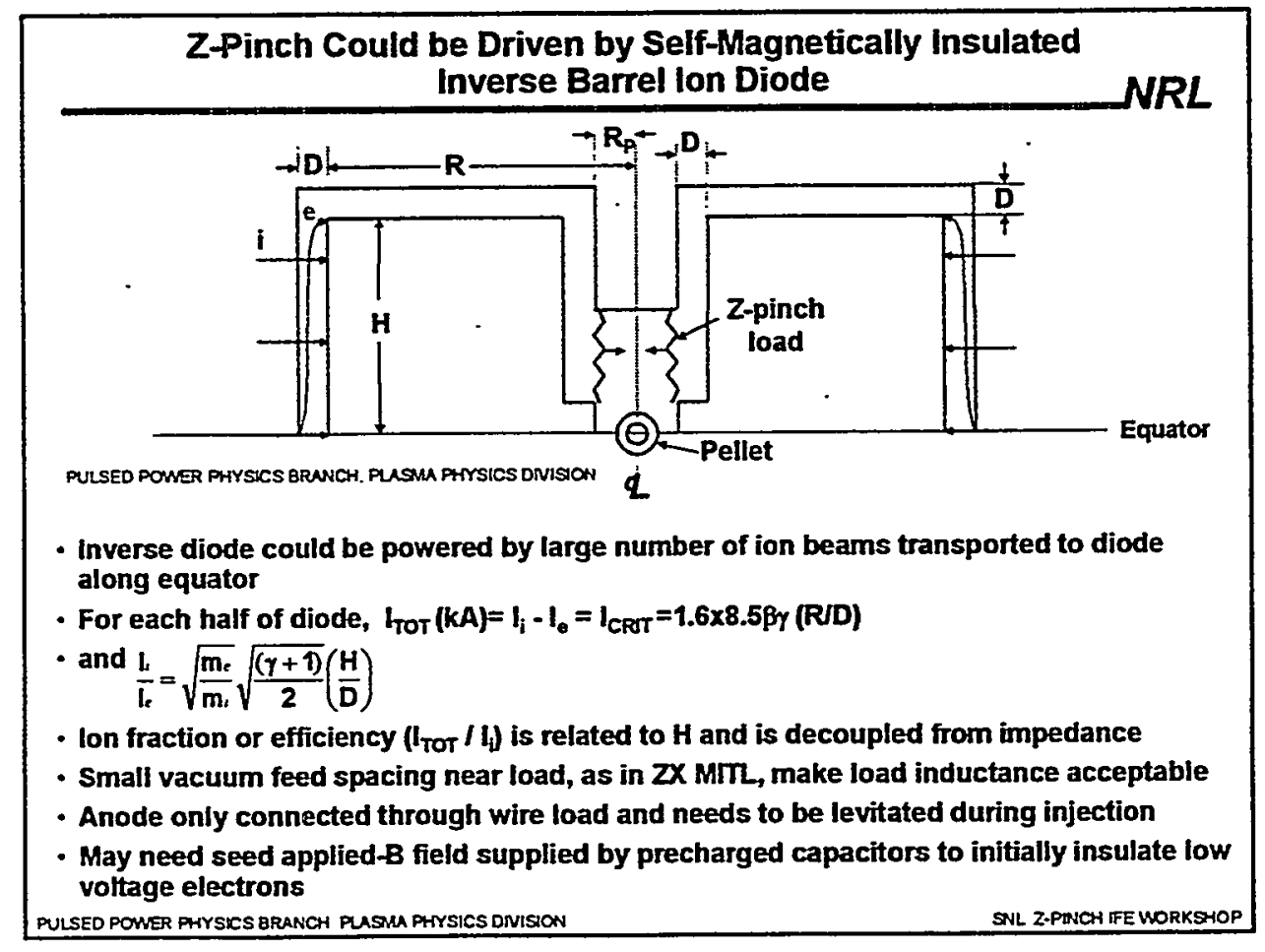




\section{Point Design}

NRL

lon Source

Assume $10 \mathrm{MV}, 100 \mathrm{MA}, 50$ ns unbunched proton beam produced by ten self-magnetically insulated $10 \mathrm{MV}, 10 \mathrm{MA}$, $50 \mathrm{~ns}$ ion diodes.

For $I_{\text {TOT }}(K A)=I_{1}+I_{e}=I_{C R T}=1.6 \times 8.5 \beta \gamma(R / D)$ and $\gamma=1+2^{\star} M V=21$

$\Rightarrow R / D=35$

Thus, for $D=0.5 \mathrm{~cm}, R=17.5 \mathrm{~cm}$

Ion fraction given by $\frac{b}{l}=\sqrt{\frac{m_{c}}{m_{i}}} \sqrt{\frac{(\gamma+1)}{2}}\left(\frac{R}{D}\right)^{\cdots}$.

Assume additional factor of 2 for reflexing.

$\Rightarrow I_{\mathrm{I}} I_{\mathrm{T}}=\mathrm{I}_{\mathrm{I}} /\left(\mathrm{I}_{\mathrm{I}}+\mathrm{I}_{\mathrm{e}}\right)=0.84$

\section{Point Design (cont)}

Inverse diode

-Assume self-magnetically insulated barrel diode with

radius $=R$, height $=H$, and $A K$ gap $=D$

-Each half of diode must operate at $10 \mathrm{MV}$ and $50 \mathrm{MA}$

-Assume $I_{\text {Tor }}(k A)=I_{i}-I_{e}=I_{C R T}=1.6 \times 8.5 \beta \gamma(R / D)$

$\Rightarrow R / D=175$

-Thus, for $D=0.2 \mathrm{~cm}$ (as in MITL feeds), $R=35 \mathrm{~cm}$

-Ion fraction given by

$$
\frac{l_{i}}{l_{e}}=\sqrt{\frac{m_{e}}{m_{i}}} \sqrt{\frac{(\gamma+1)}{2}}\left(\frac{H}{D}\right)
$$

-For efficient capture, ássume $I_{T} l_{i}^{2}=\left(l_{i}-1_{e}\right) n_{i}=0.84$

$\Rightarrow I, n_{e}=6.25$ and $H / D=81$

-Thus, for $D=0.2 \mathrm{~cm}, H=16 \mathrm{~cm}$

-Target and source size comparable and target easy to hit

-Dominant inductance is $L \approx 2 \mathrm{H}$ In $\left(\left(R_{\mathrm{p}}+D\right) / R_{p}\right)$ where $R_{p}=$ initial array radius

-Thus $L \approx 6 \mathrm{nH}$ for $R_{P}=1 \mathrm{~cm}, D=0.2 \mathrm{~cm}$

$\Rightarrow \mathrm{Ldl} / \mathrm{dT}=6 \mathrm{MV}$ 


\section{Point Design (cont)}

Source divergence and transport

-For ballistic transport

-Assume source focuses onto target $5 \mathrm{~m}$ away

-Since target height is $\pm 16 \mathrm{~cm}$, allowed source divergence is $\theta= \pm 32 \mathrm{mrad}$

- Ballistic focusing followed by self-pinch transport would allow even larger source divergence

-For self-pinch or guided transport

$I_{c h}=I_{A} \theta^{2}$ and

$I_{A}=31.3 \beta_{1 y_{1}}(A / Z)=4.57 \mathrm{MA}$ for $10 \mathrm{MV}$ protons

-Thus, $I_{c h}<200 \mathrm{kA}$ for $\theta<200 \mathrm{mrad}$

\section{Ion Source Options}

- Self-magnetically insulated ion diodes

- self-pinched electron beam diode enhances electron lifetime by R/D

- pinch-reflex diode enhances electron lifetime via magnetic reflexing

- "backless" or low-mass-anode diode enhances electron lifetime via electrostatic reflexing from virtual cathode

- for all of above, magnetic bending or defocusing due to AK gap closure is partially compensated for during pulse by decreased bending distance

- Applied-B extractor ion diode

- MAP Applied-B extractor ion diode with ionized gas anode

- Higher-Z ions from two-stage applied-B extractor ion diode with stripping to enhance current in inverse diode - suggested by Slutz

- All of above need preformed active anode plasma through plasma injection or gas ionization

- All of above could possibly benefit by active plasma fill with AK gap naturally forming in plasma sheaf 


\section{Ion Transport Options}

- Ballistic transport

- plasma created by beam-induced gas breakdown provides charge and current neutralization

- beam can be ballistically transported and focused with solenoidal lens

- Z-discharge transport in preformed plasma channel

- beam charge and current neutralized with confinement provided by discharge current

- laser initiated channels as considered in HIF schemes

- low-mass wall-confined channels

- Self-pinched transport

- requires plasma created by beam-induced gas breakdown to provide total charge and partial current neutralization

- net beam current provides beam confinement

- Wire guided transport

- beam charge and current neutralized with confinement provided by wire current

- Ballistic transport and focusing can be followed by guided transport

\section{Inverse lon Diode Options}

NRL

- Self-magnetically insulated barrel diode

- because of complex electron orbits, it might be possible to exceed critical current leading to smaller diodes

" need PIC simulation

- may require seed applied-B field to initially insulate low voltage electrons n could be supplied by small precharged capacitor attached to diode

- Applied-B magnetically insulated barrel ion diode

- magnetic field could be supplied by larger precharged capacitor

- virtual anode can be neutralized by electrons leaking across magnetic field lines as suggested by Slutz et al

- ion current density limitations might make diode dimensions unreasonable

- Both diode options could be powered by large number of ion beams transported to diode along equator

- Coaxial versions of both diodes could easily be tested on present facilities 
Issues

NRL

- To numerous to elaborate

- Major issue is survivable rep-rated ion diode

- need proof-of-principle experiments coupled with numerical simulation for scaling 


\section{Development of a \\ Low-Technology Fusion Reactor}

(or a zero miracle fusion reactor)

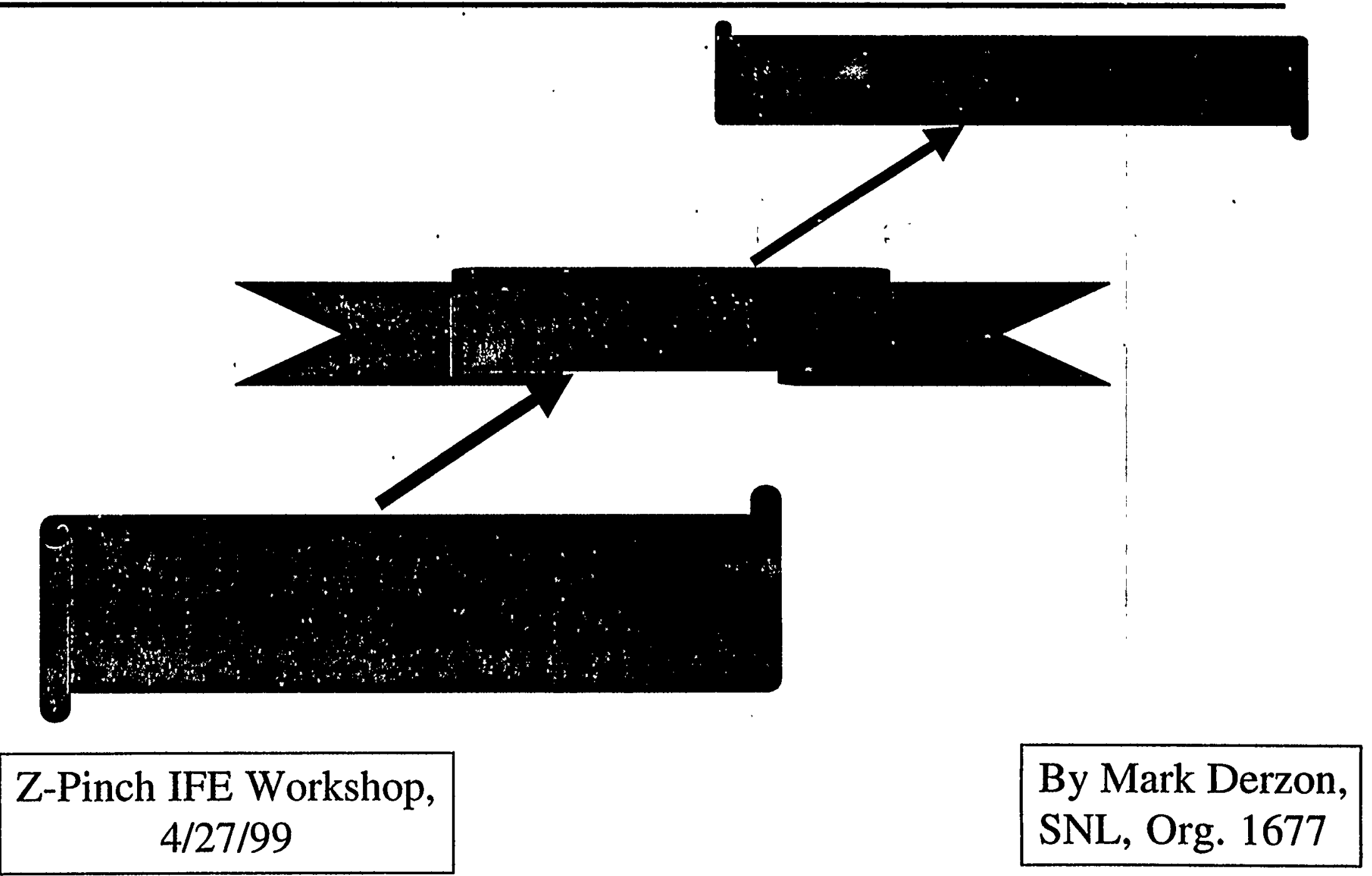




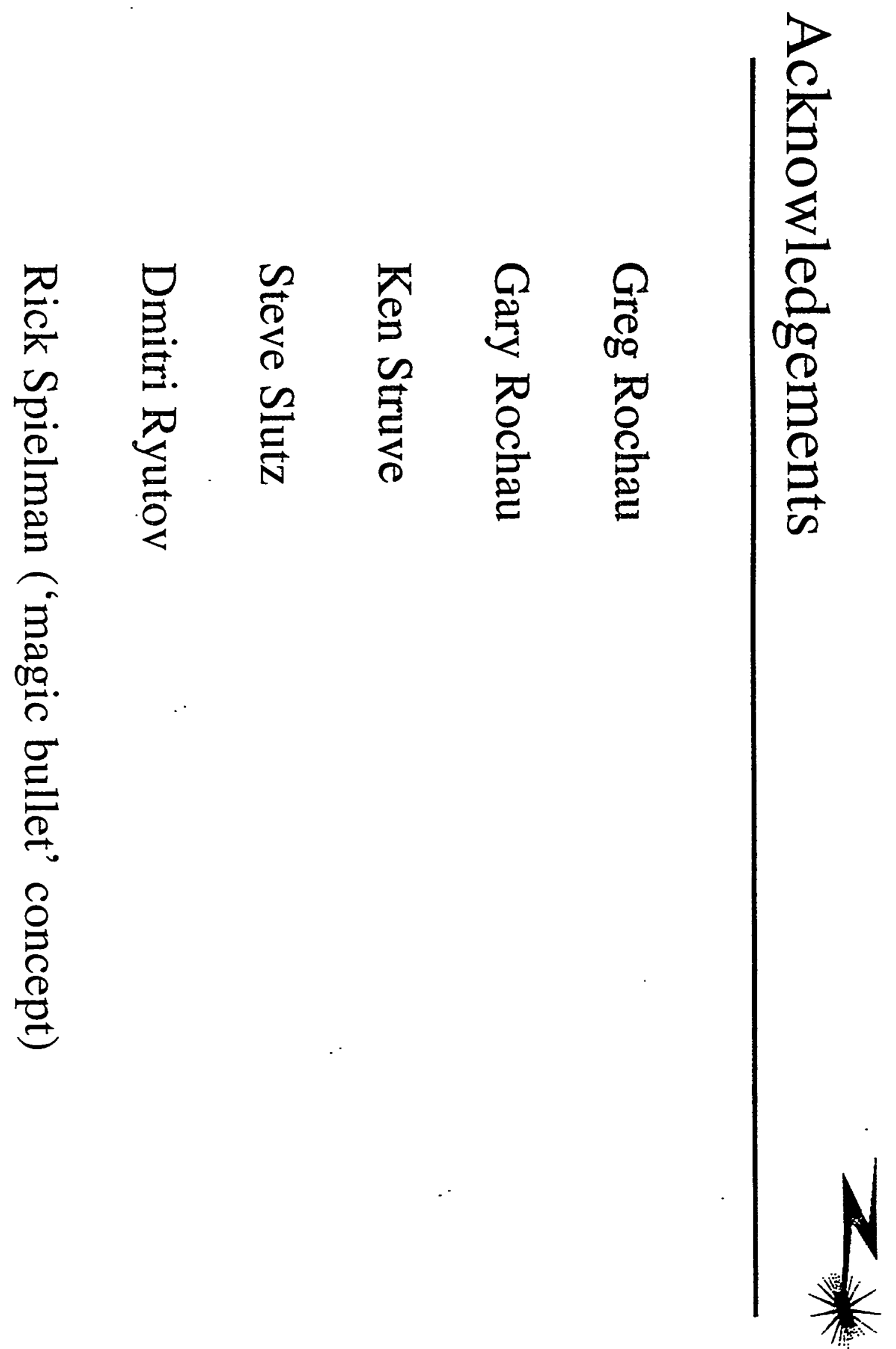




\section{Agenda}

\section{Basic considerations}

Tritium Breeding

Wall Survivability

Optimal Yield

Current Source 


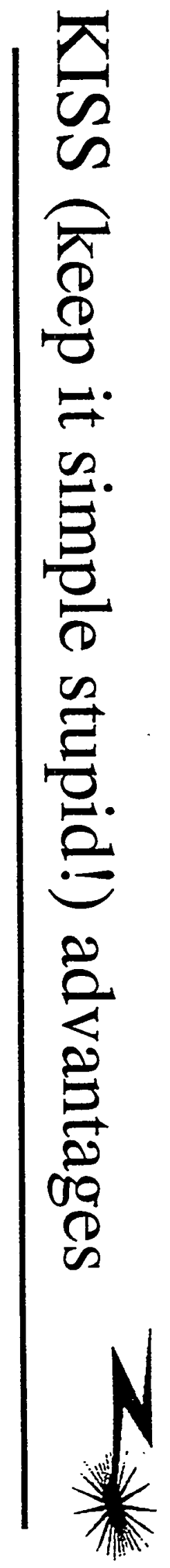




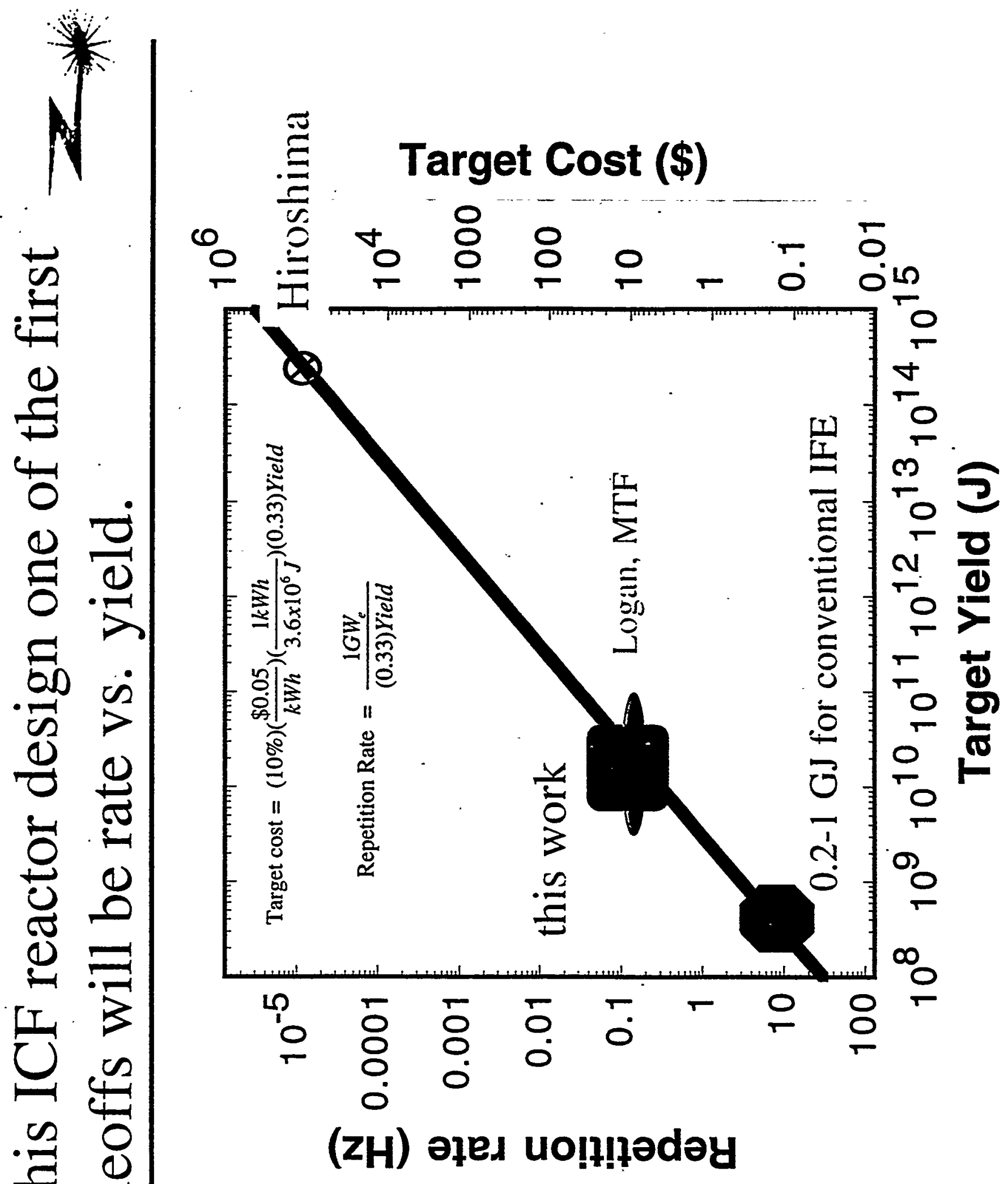




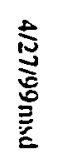
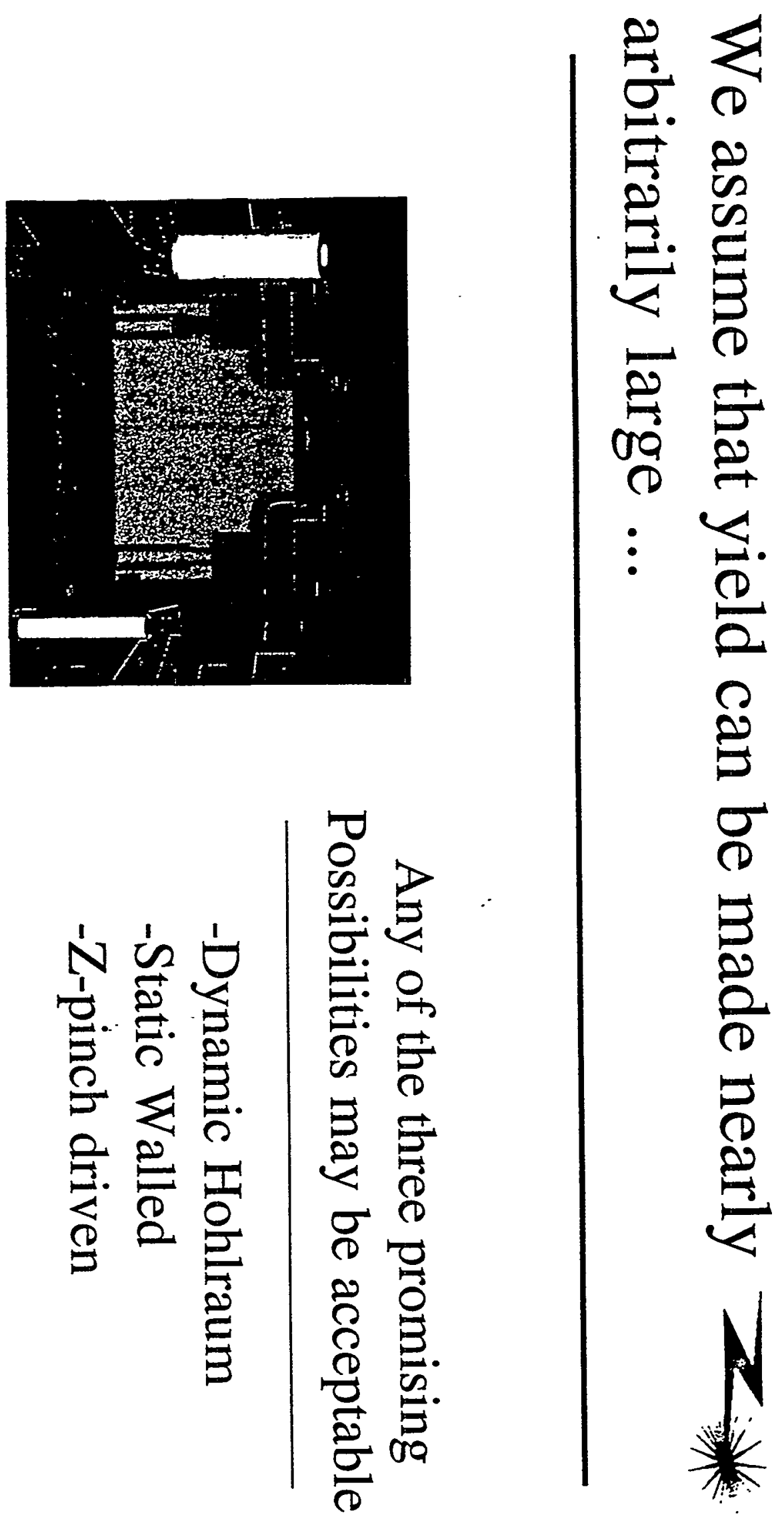


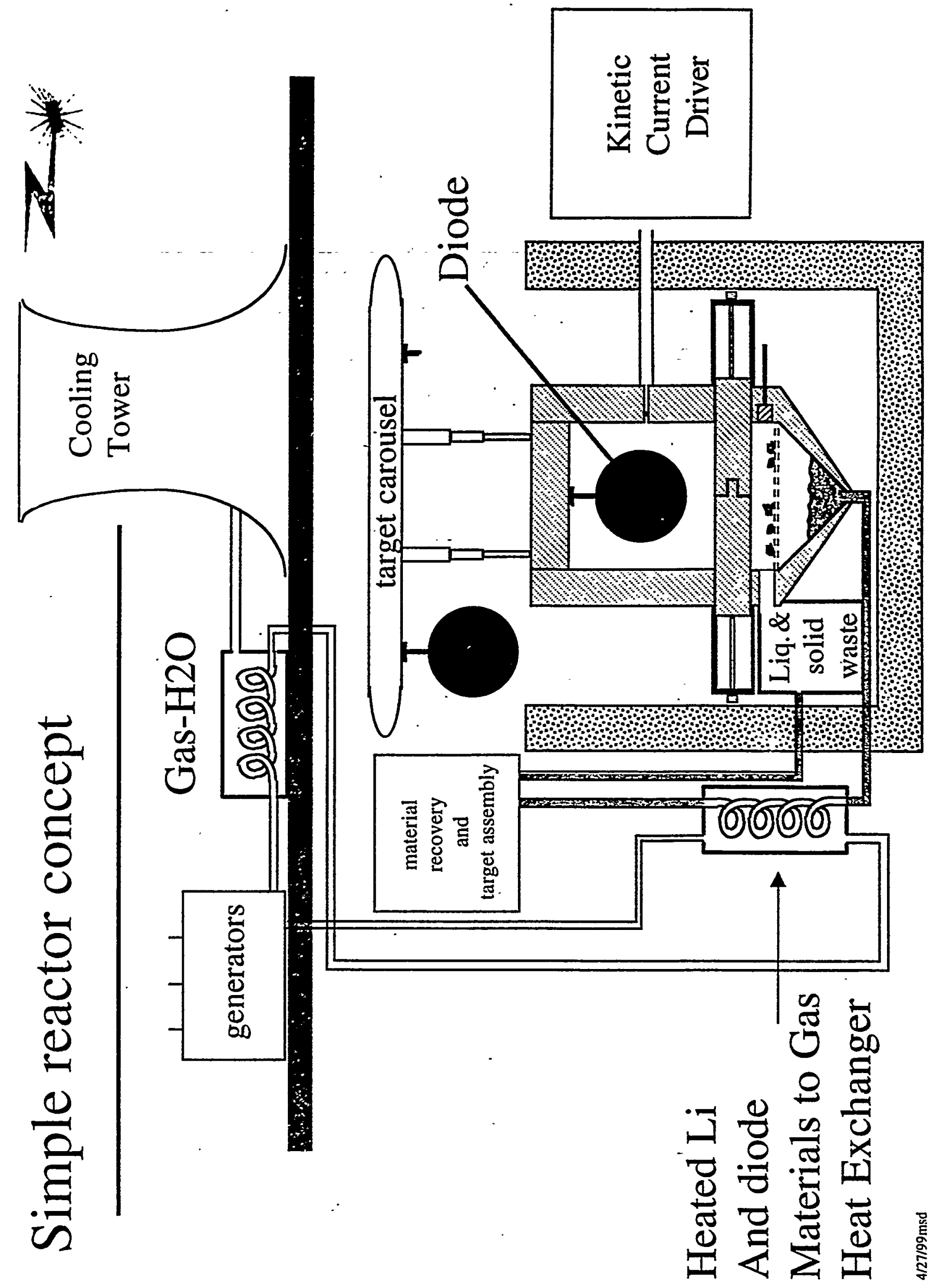


节

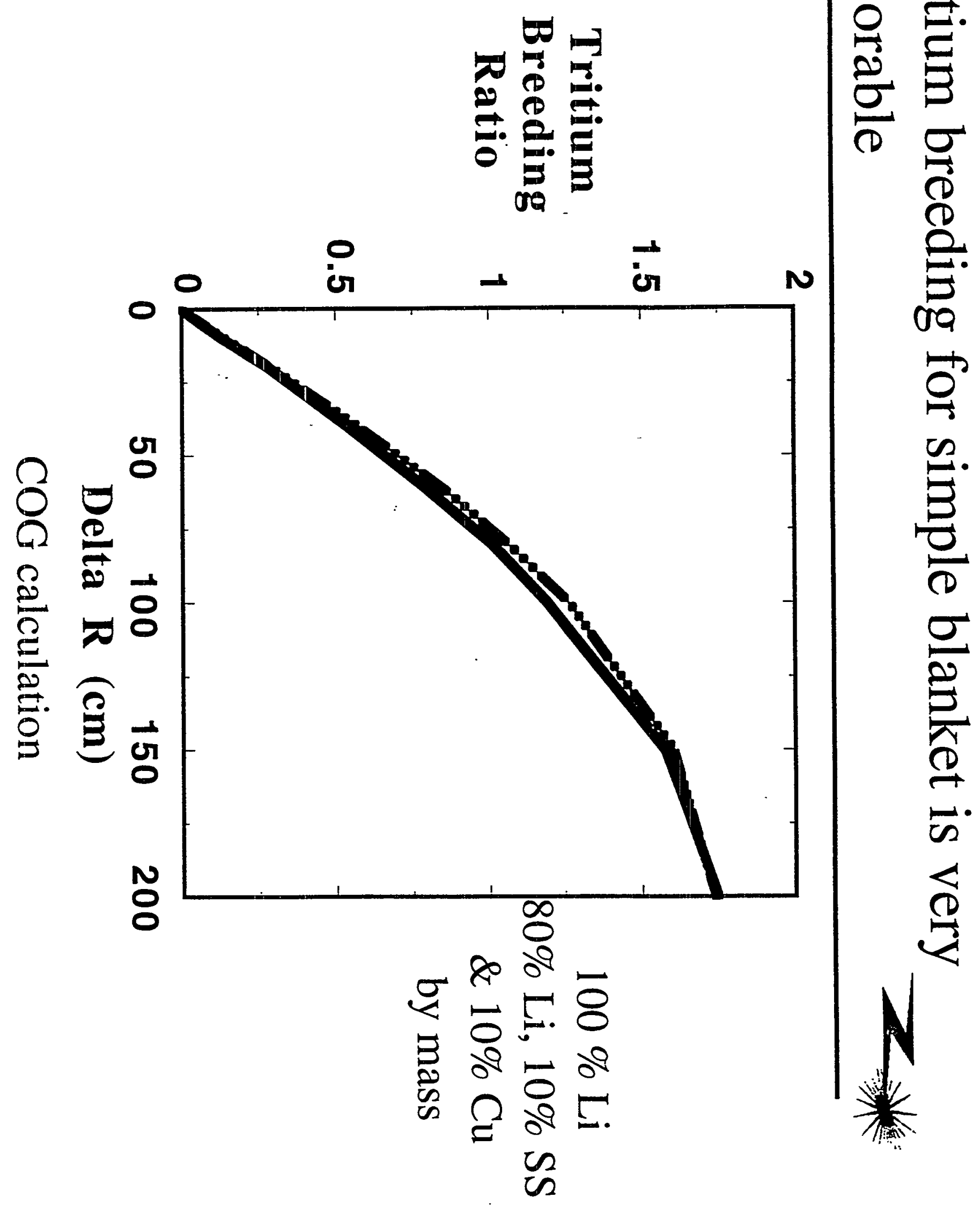




\section{Wall flux sets minimum of confinement vessel size}

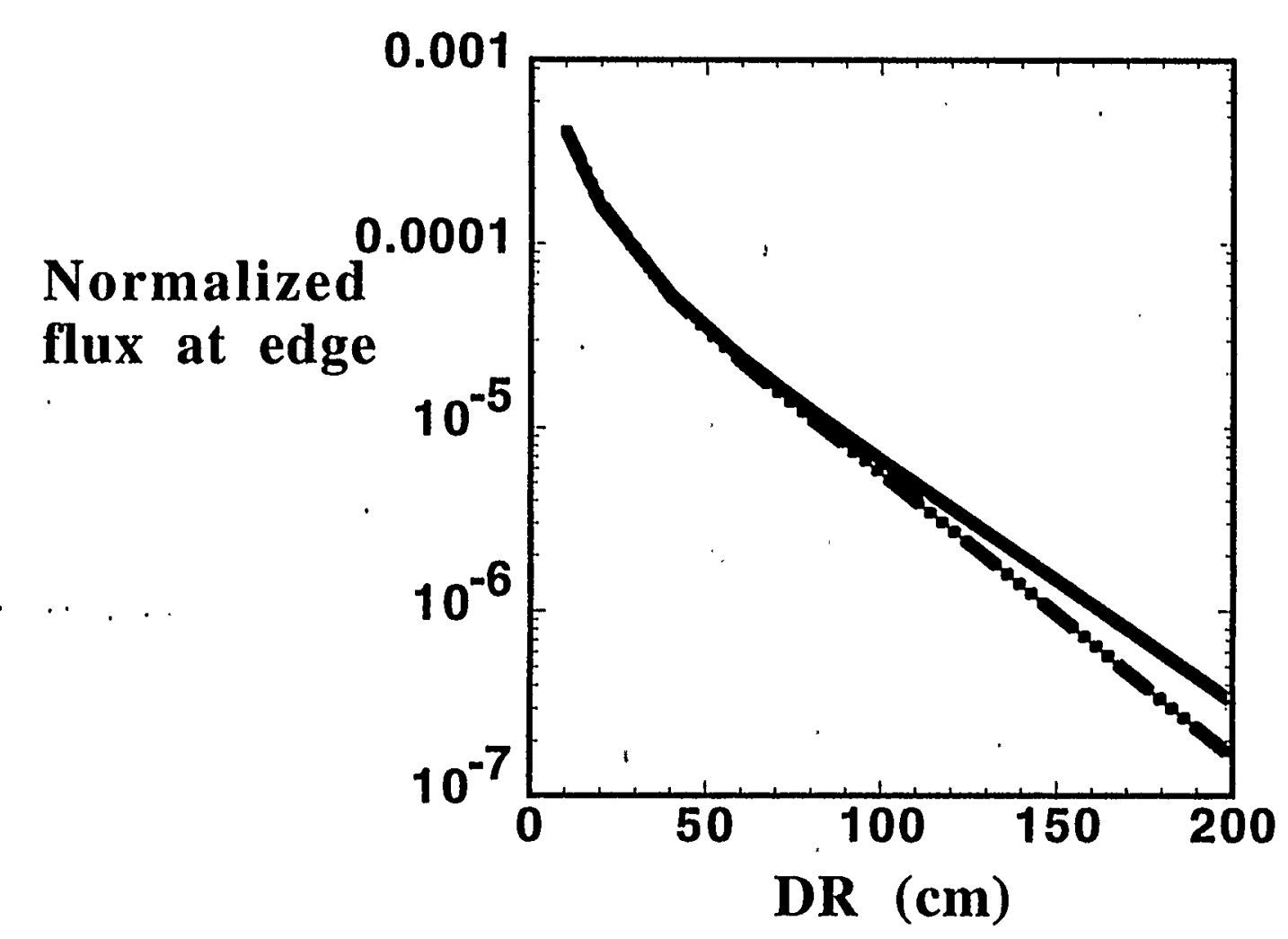

$$
\begin{gathered}
3 \times 10^{22} \mathrm{n} / \mathrm{cm}^{2} \\
\text { limiting fast-neutron } \\
\text { wall flux sets } \\
\text { survivability, } \\
\mathrm{F}\left(\mathrm{cm}^{-2}\right)=\frac{S\left(y r^{-1}\right)}{4 \mathrm{p} R_{c}(\mathrm{~cm})^{2}} f_{\mathrm{F}} t_{w}(y r) \\
\frac{f_{\mathrm{F}} t_{w}}{R_{c}^{2}}<2 \times 10^{-9} \mathrm{yrcm}^{-2}
\end{gathered}
$$

With $\mathrm{DR}=150 \mathrm{~cm}$, and chamber radius, $\mathrm{R}_{\mathrm{c}}=2 \mathrm{~m}$ the wall survives $\sim 50$ years 

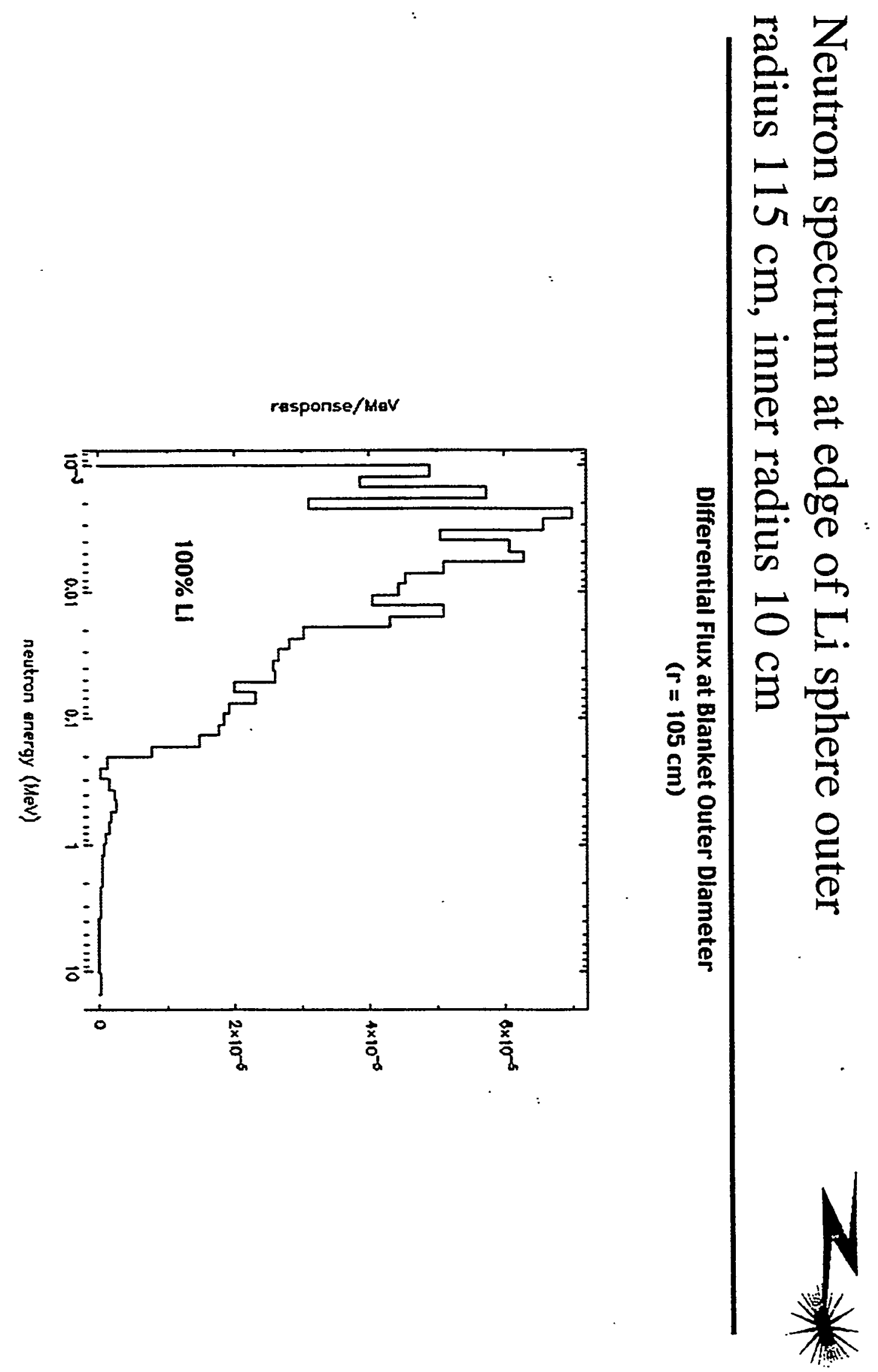

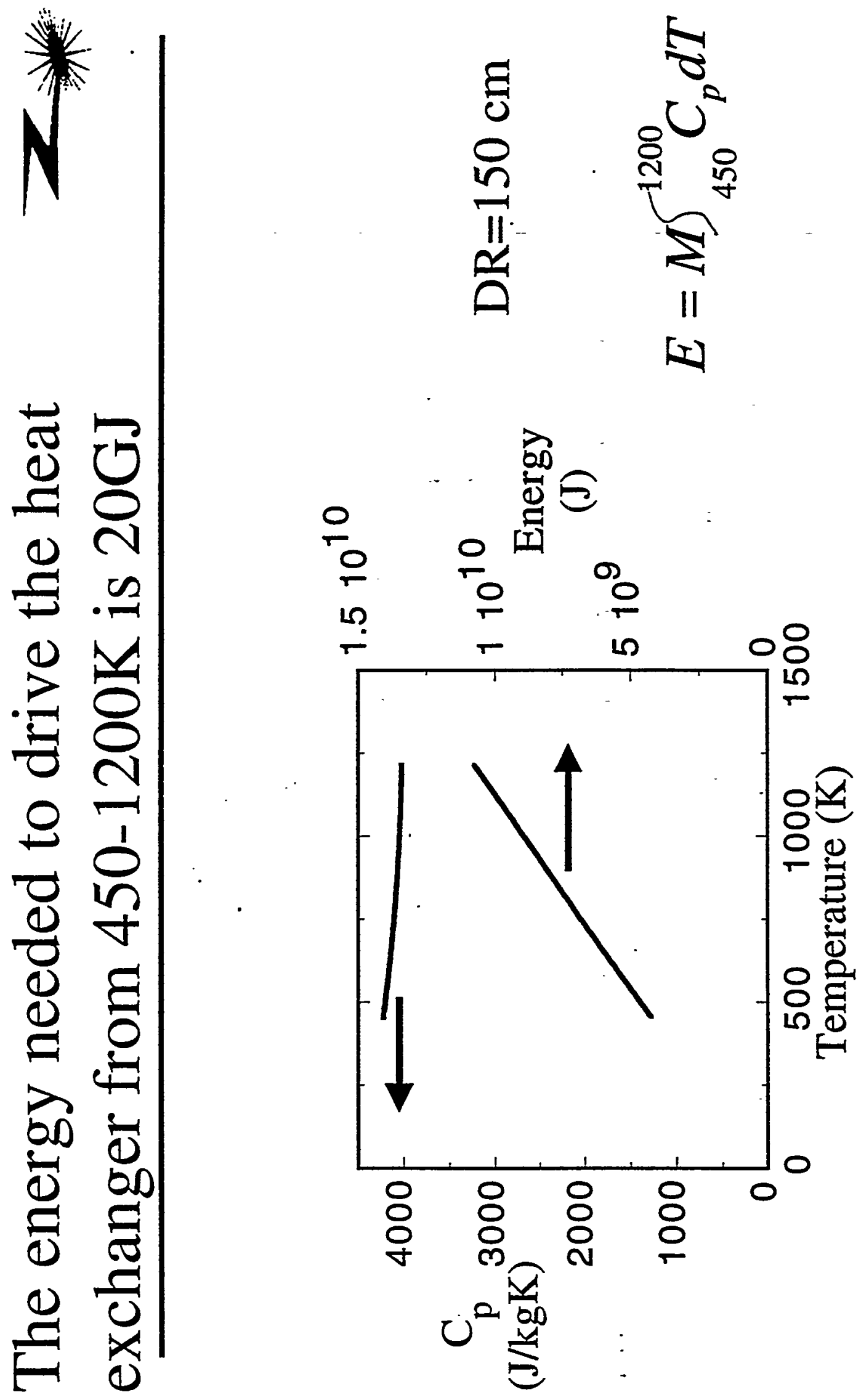
I
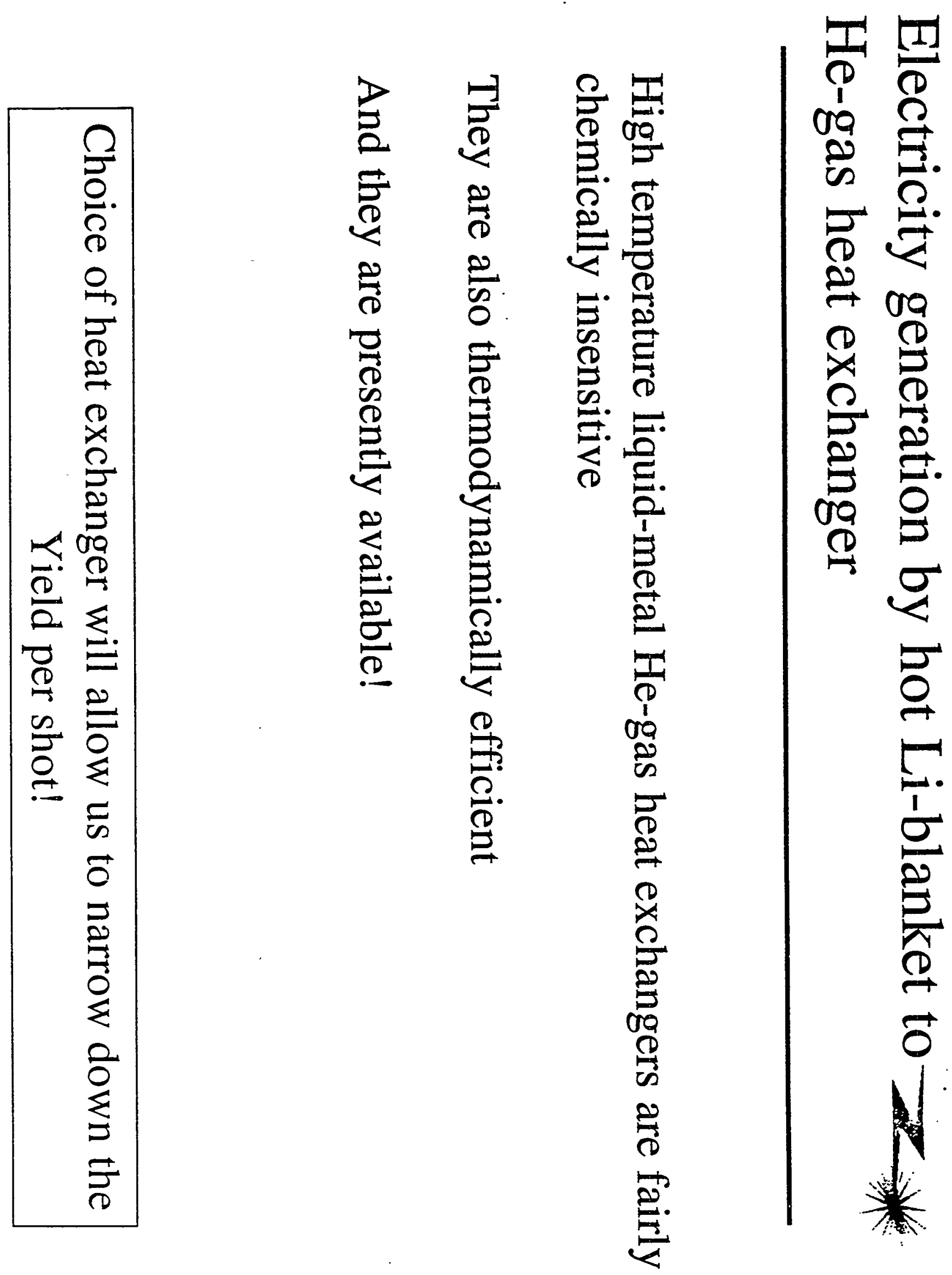


\section{How do you confine a 20 GJ (5 Ton TNT) explosion?}

Presently $1000 \mathrm{~kg}$ TNT confined in $16 \mathrm{~m}$ diameter vessels with $20 \mathrm{~cm}$ thick shells at Russian Academy of Sciences in Moscow, Institute for High Temperature.

DAHRT technology has been demonstrated for total containment At $45 \mathrm{~g}$ of TNT in a 18 " diameter $3 / 8$ " wall chamber.

Both technologies scale to $10-20 \mathrm{GJ}$ : $60-90 \mathrm{ft}$ diameter, 16 " wall chamber.

- Without mitigation, conservative design.

Use compressible material between explosion and vessel to mitigate Blast. (Li foam?) 


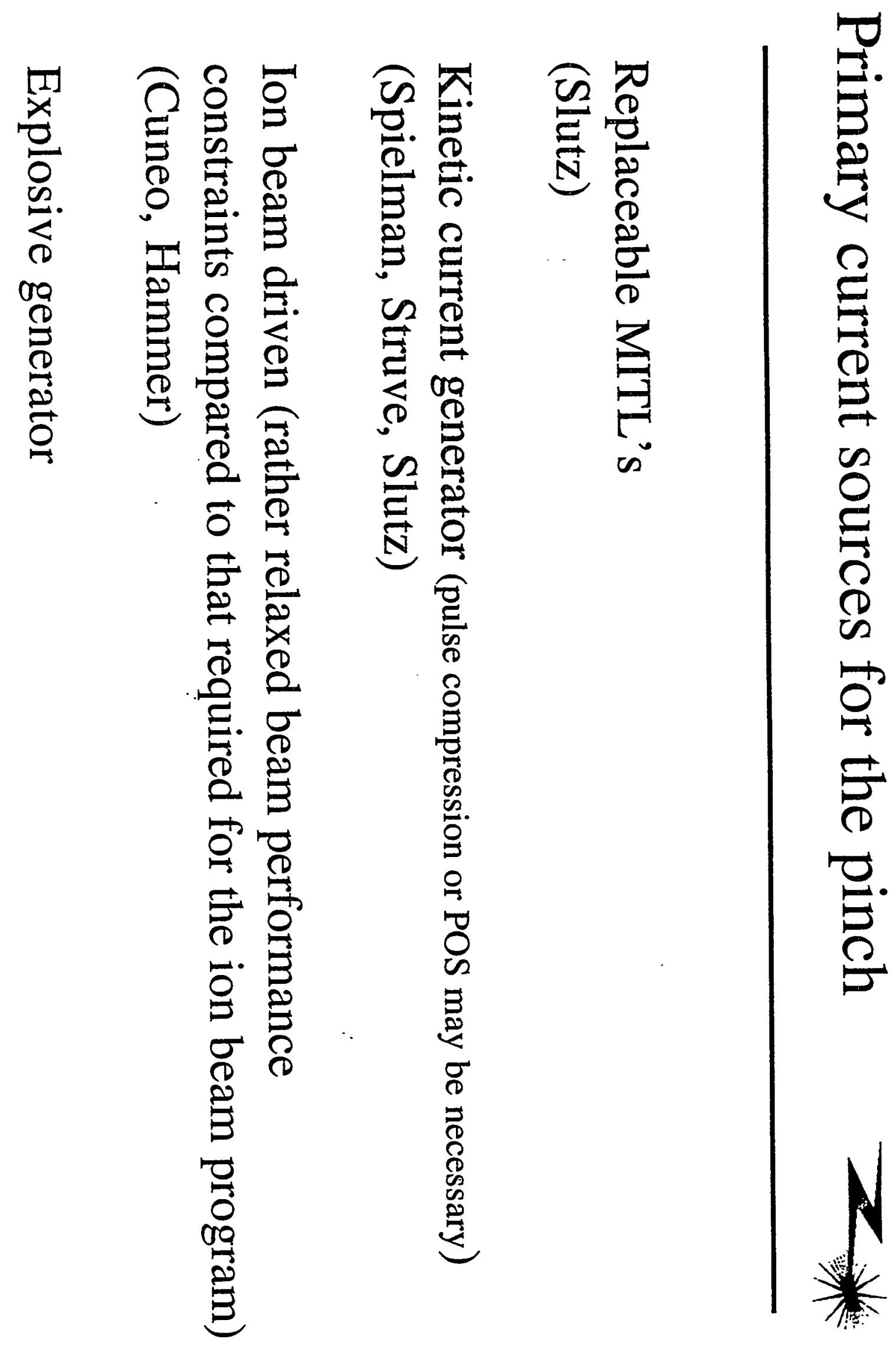


Kinetic current generator passes a 'bullet' into a magnetic field, current is created when field is excluded
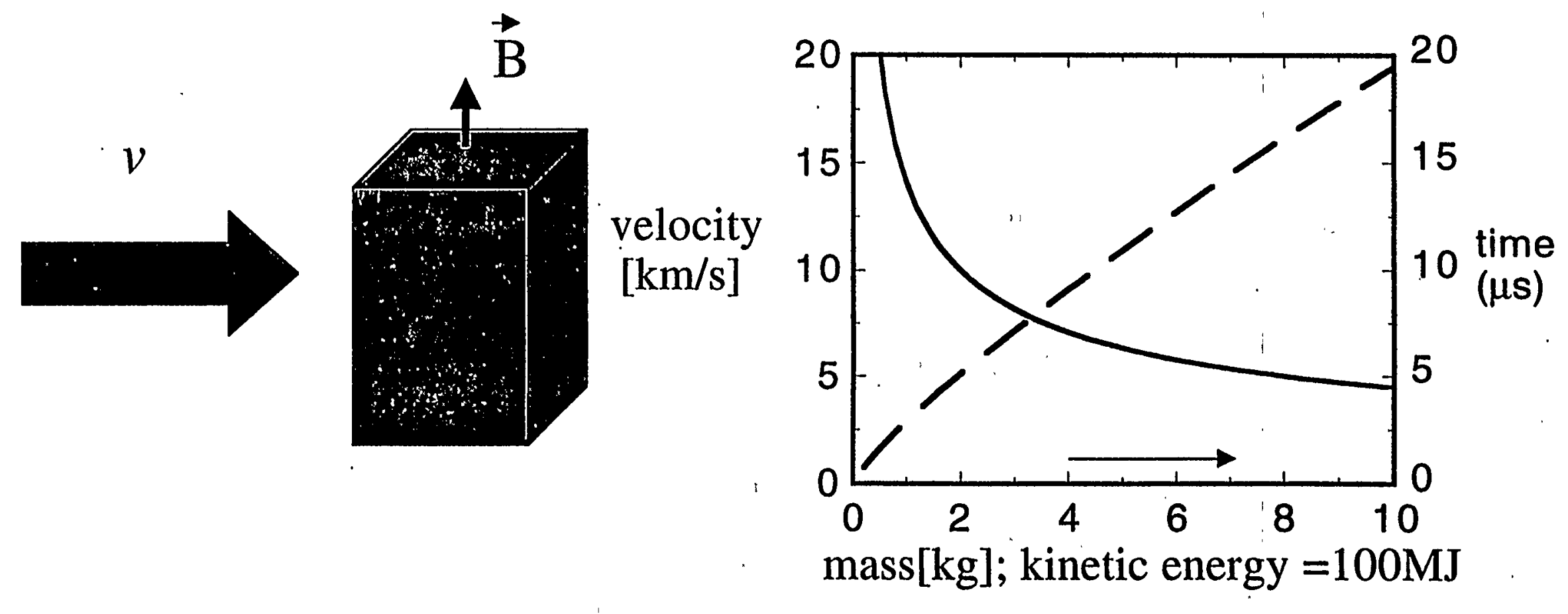

right-circular cylinder bullet

Pulse risetime and energy transfer efficiency may be limiting factors. POS or pulse compression may be required. 

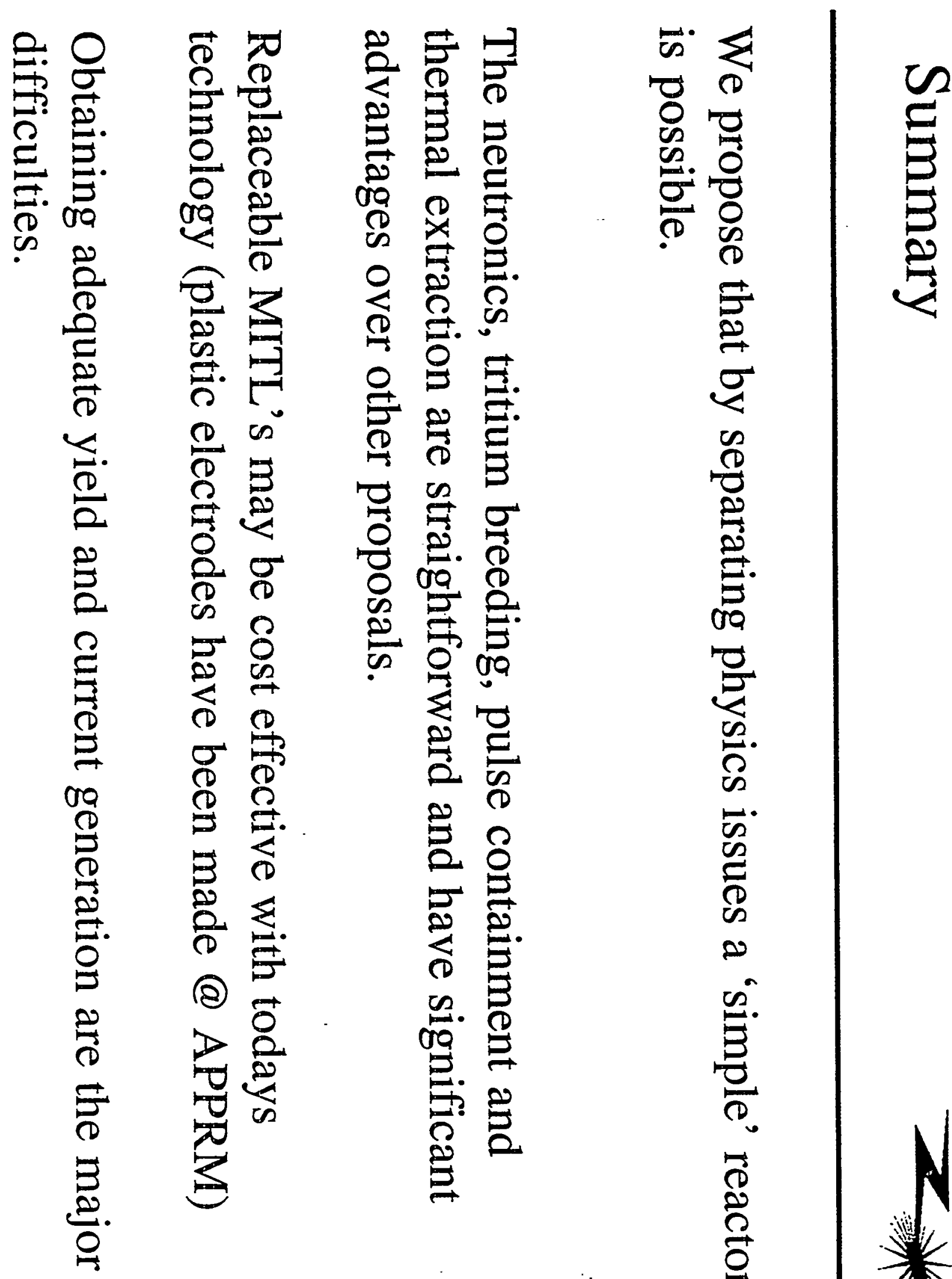


\section{Target Chamber Options for Z-Pinch IFE}

G.L. Kulcinski, R.R. Peterson, D.C. Kammer, H.Y Khater, E.A. Mogahed, G. Rochau, J.F. Santarius, M.E. Sawan, I.N. Sviatoslavsky, T. Utschig Z-Pinch IFE Workshop

Sandia National Laboratories Albuquerque, NM April 27 - 28, 1999 
Many Options for Target Chambers to Contain High Debris

\section{Mass Blasts}

Several options considered

-Dry Wall Gas-Protected

•HIBALL Wetted Wall

-HYLIFE-II Liquid Wall

-Swirling Liquid Vortex around Target

-Thick Liquid Absorber on Target
Target Chamber for Z-Pinch

IFE needs to:

-Maintain vapor/gas density consistent with power flow.

-Protect permanent structures from target $\mathrm{x}$-rays and debris ions and shrapnel from power feed system.

- Recover energy.

-Manage radioactivity production and containment. 


\section{Dry Wall Target Chambers With Gas Protection are}

Compatible with High Chamber Rep-rate.

-SOMBRERO concept has been applied to direct drive laser fusion.

-Gas stops target X-rays and debris ions and forms a fireball.

-Best suited to small yields or large target chambers.

-Not compatible with large shrapnel mass.

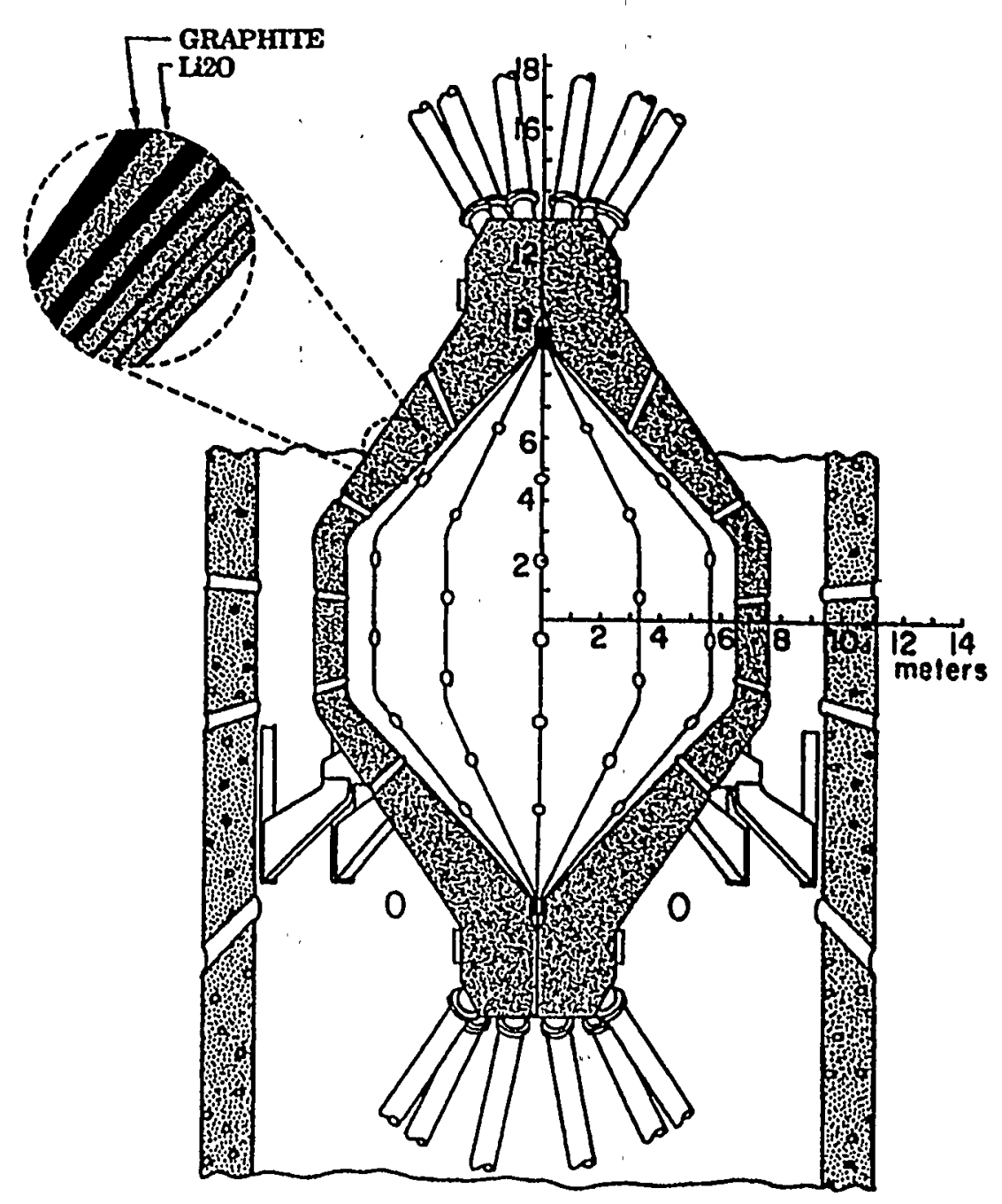

Fusion Technology Institute

University of Wisconsin - Madison

\section{Z-Pinch IFE}




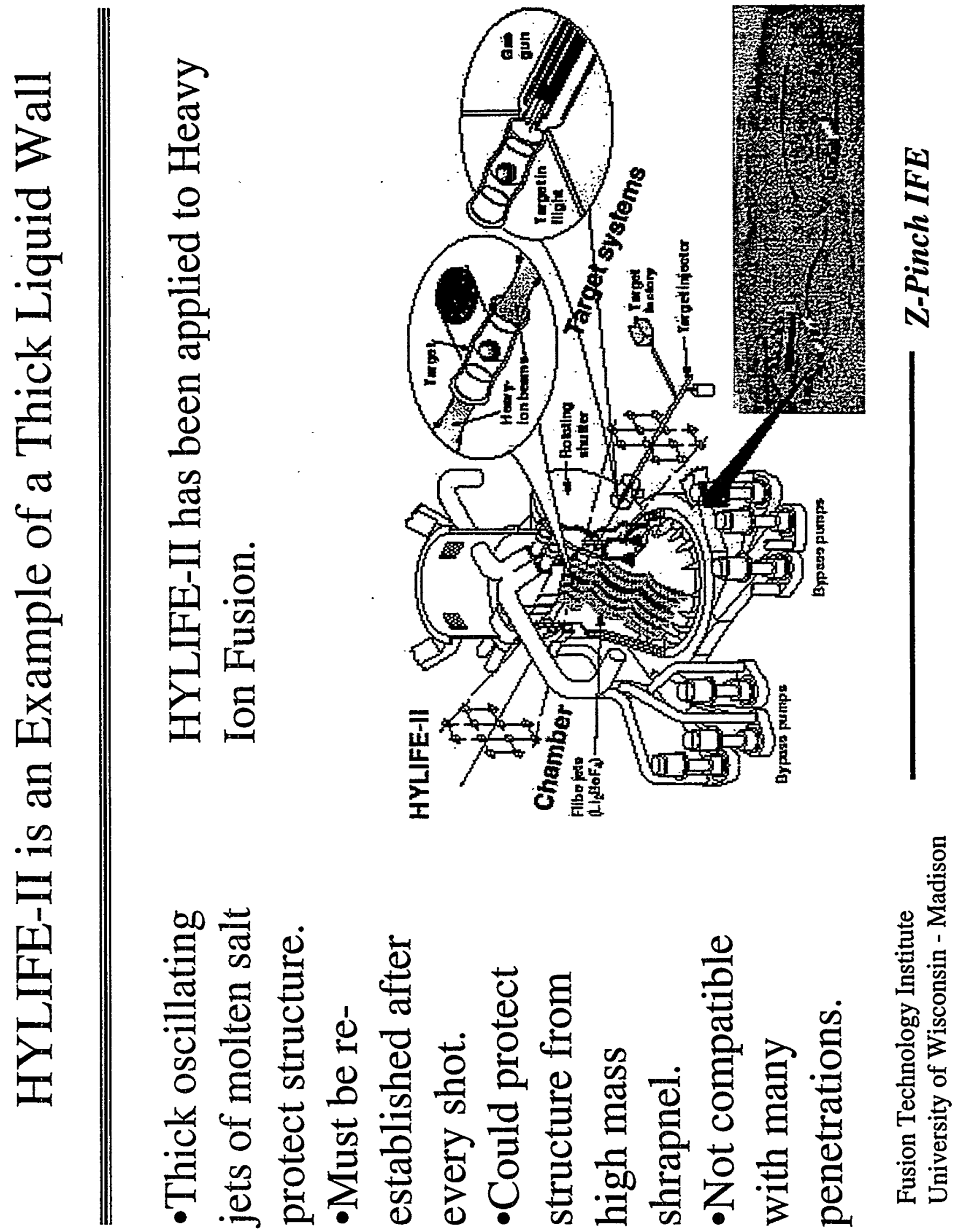




\section{Wetted-Wall Target Chambers, such as HIBALL, Allow High Yield and Several Penetrations}

-HIBALL uses liquid flowing in continuously wetted tubes to protect the first wall from neutron damage.

- Wetting protects the tubes from large $\mathrm{x}$-ray and debris fluences.

-Moderately compatible with large mass shrapnel.

-Rep-rate limited by vaporization and condensation.

Fusion Technology Institute University of Wisconsin - Madison
The HIBALL target chamber concept has been applied to Heavy Ion Fusion. The Light Ion LIBRA concepts also use liquid confined to wetted tubes.

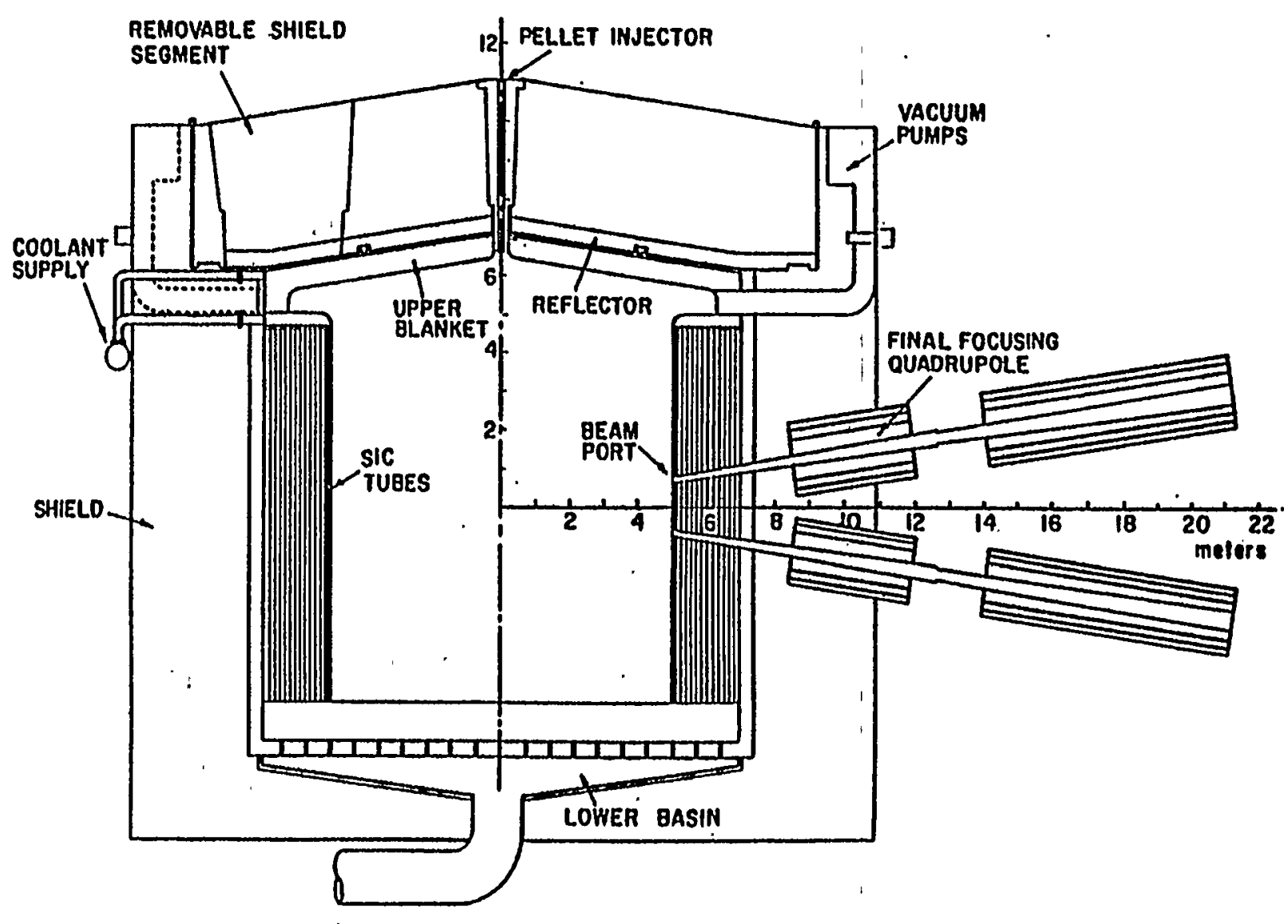




\section{Swirling Vortex of Liquid Metal Captures Target X-ray, Debris and Neutrons in a Liquid Near the Target}

-Applied to MCF z-pinches.

-Current carried by liquid metal.

-Thick liquid metal absorbs fusion products.

-In z-pinch IFE, liquid metal would absorb target mass.

-Spinning liquid re-establishes geometry prior to each shot.

-Not compatible with ion beam electrical feeds.

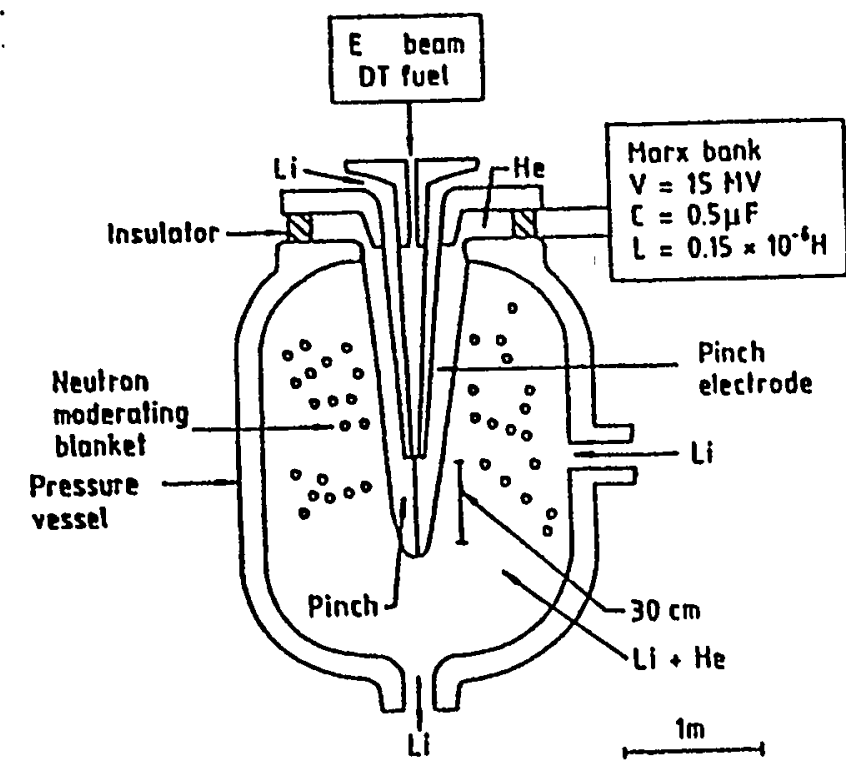

Hartman, et al., Nucl. Fusion 17,

(1977) 


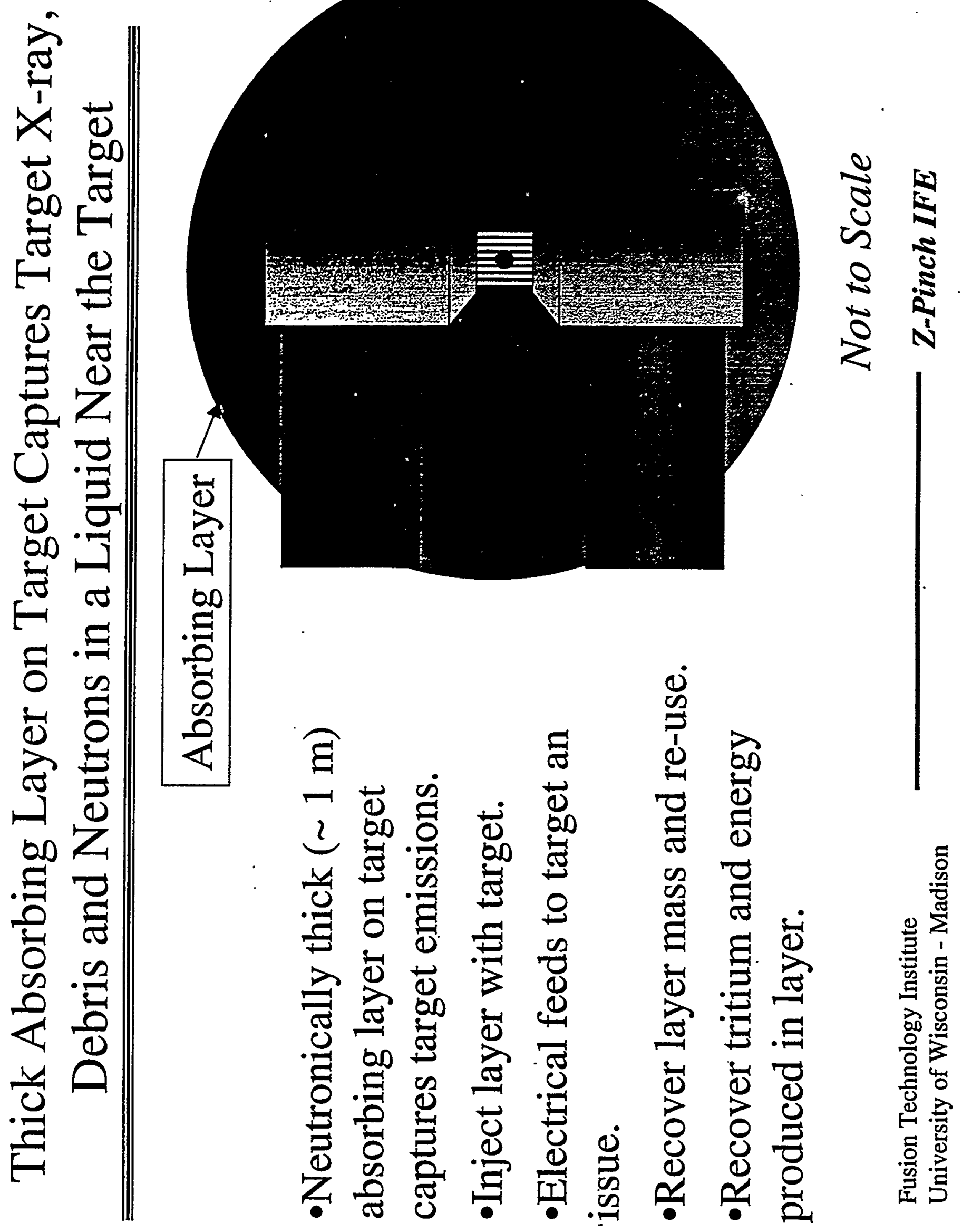




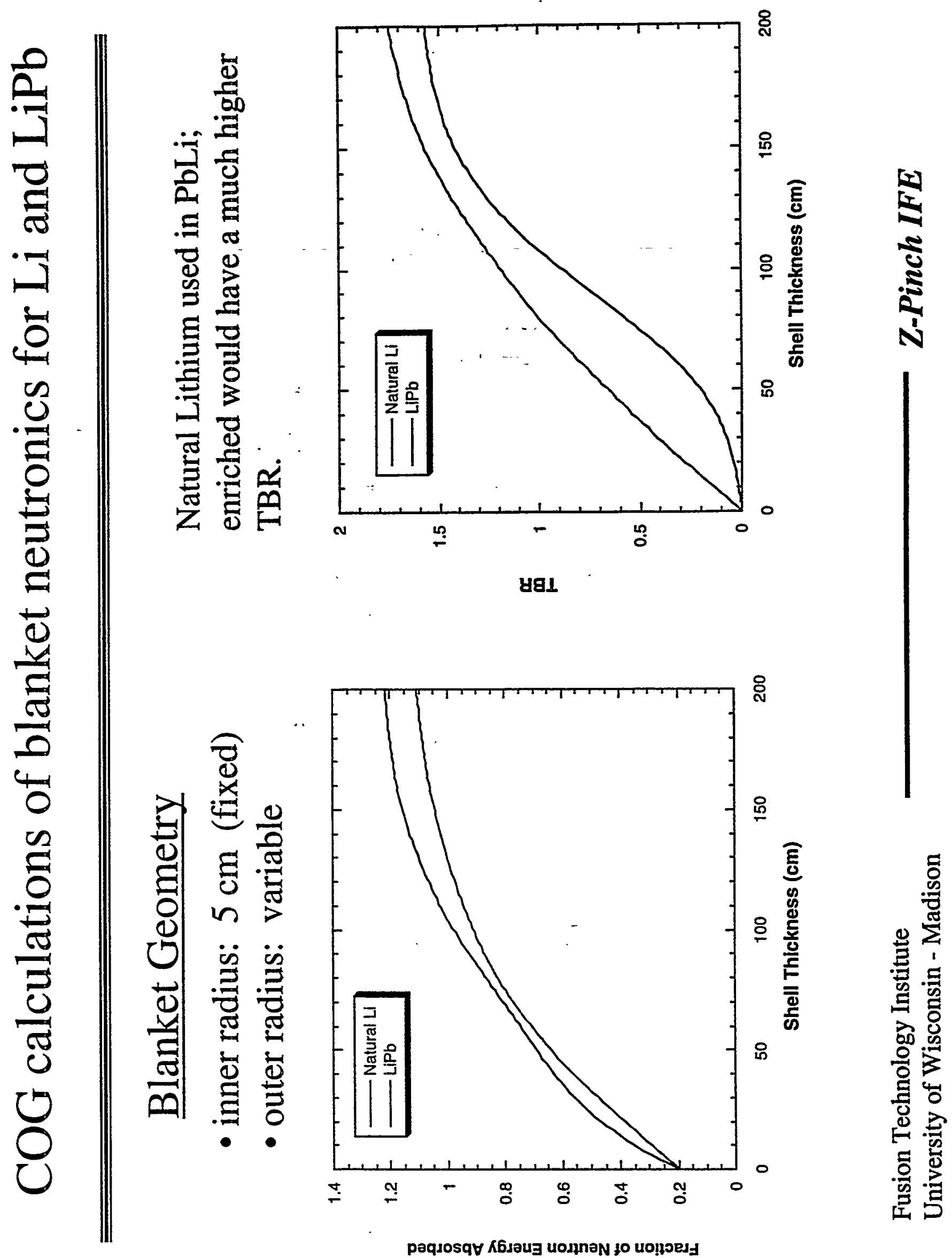




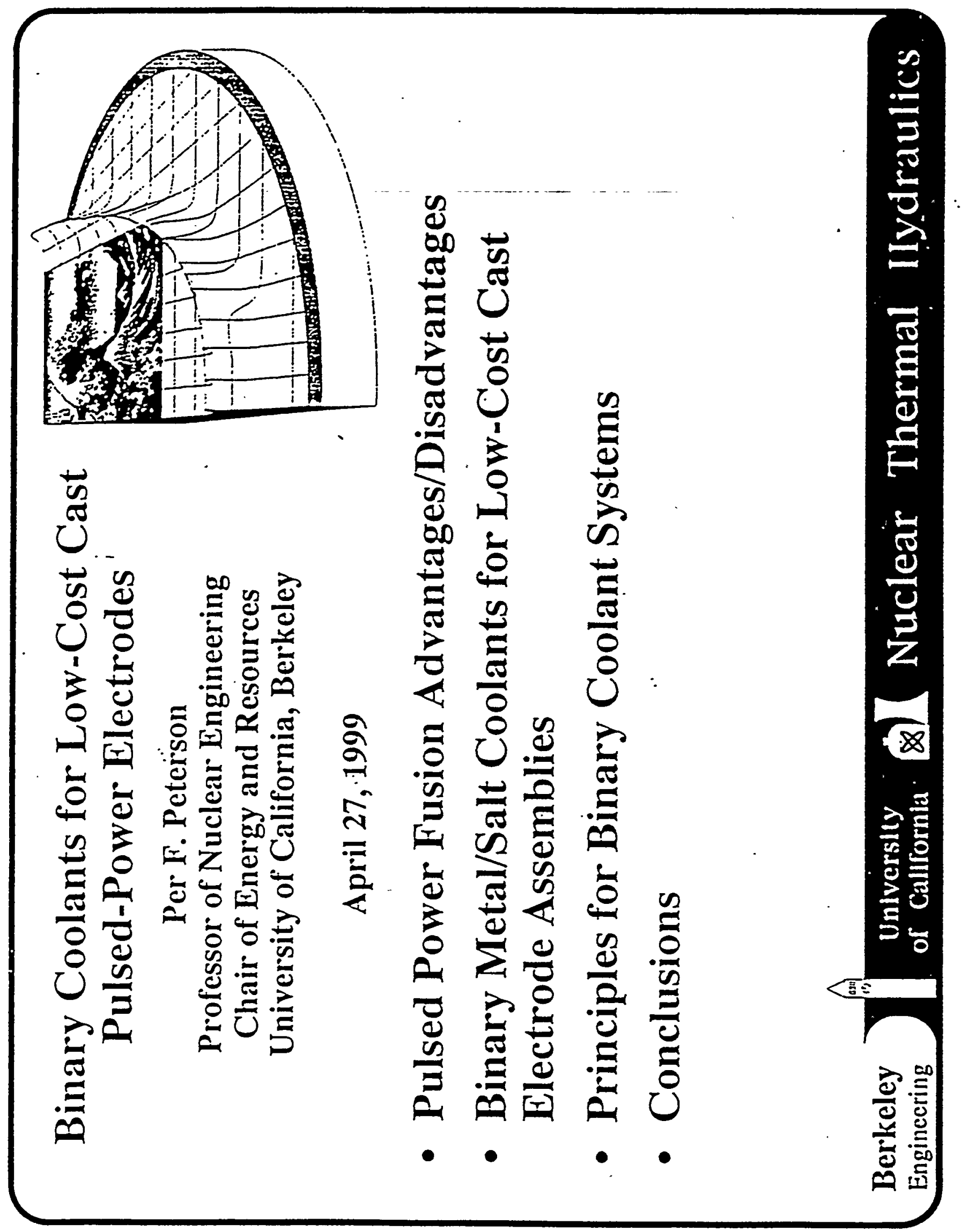




\section{Pulsed Power Fusion Advantages/Concerns}

- Advantages

- Relative simplicity of achieving standoff using electrodes compared to lasers/heavy-ion drivers

- Readily adapted to liquid protection and long structure life

- Potentially very low cost of pulsed power compared to tokamak superconducting magnets and laser/heavy-ion drivers

- Concerns

- Cost of electrode and target fabrication - The Kopeck Problem

- Rapid changeover $(>0.25 \mathrm{~Hz})$ of targets

- Viability of achieving sufficient gain for relatively low repetition rates $(0.25 \mathrm{~Hz},>1-\mathrm{GJ}$ yields) compared to $\mathrm{HI} /$ laser IFE

- Controlling blast effects from high yield targets 


\section{Binary Coolants for Pulsed Power: Principles}

- Draw on liquid-protection research for IFE: HYLIFE

- Neutron shielding, corrosion, tritium recovery, balance of plant issues studied extensively for Flibe molten salt coolant

- Search for salt-metal combinations with the following desirable properties

- Lithium bearing for tritium breeding (Flibe provides)

- Melt temperatures between $200<\mathrm{T}_{\text {melt }}<500{ }^{\circ} \mathrm{C}$

$»<500{ }^{\circ} \mathrm{C}$ for materials compatibility and freeze prevention

$»>200{ }^{\circ} \mathrm{C}$ for useful energy production from heat of fusion

- Chemically compatible and immiscible with molten salt

- Reasonable activation and waste disposal properties

- Reasonable casting and solid mechanical properties

- Reasonably low metal electrical resistivity 


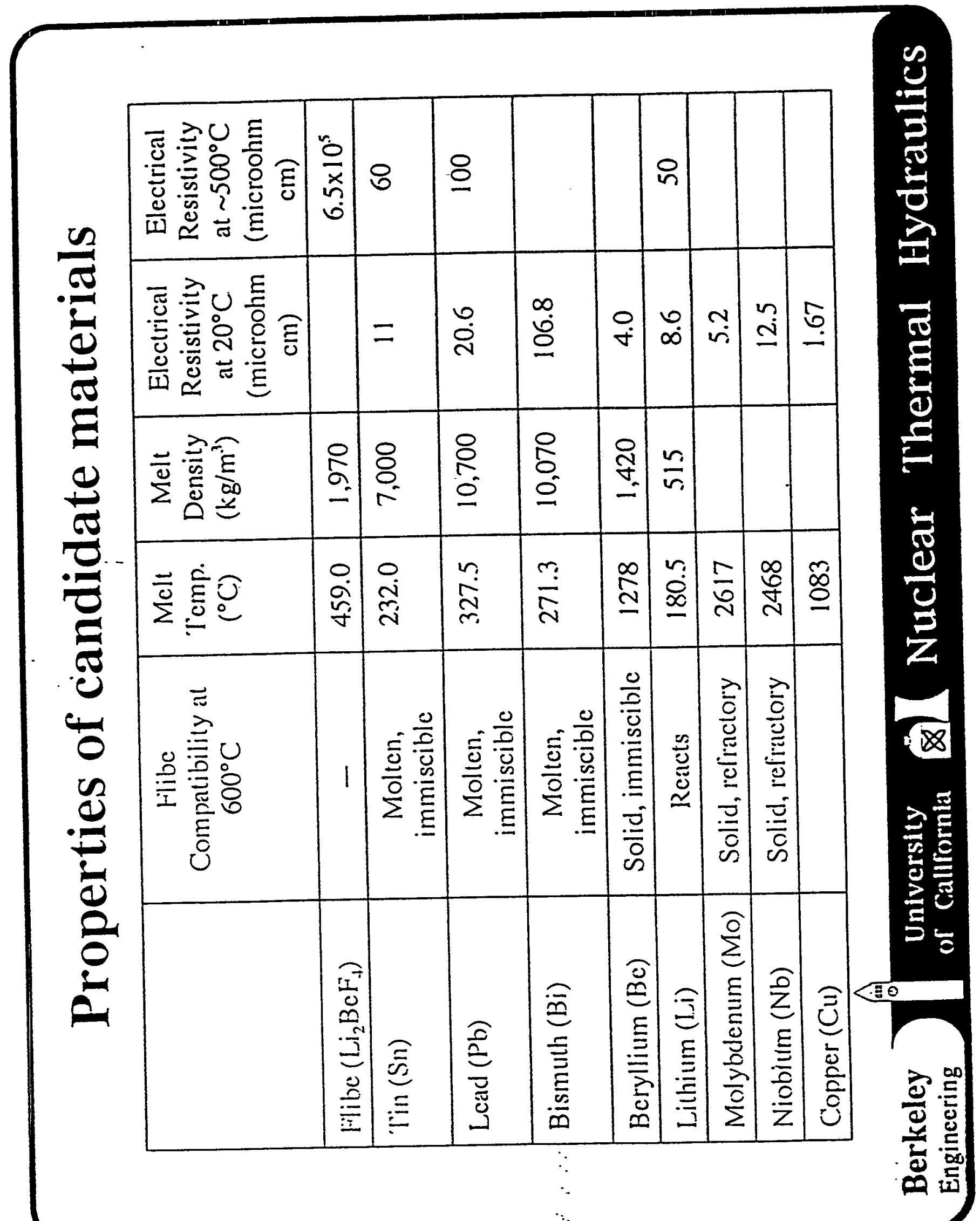




\section{Binary Coolant Fusion Plant Schematic}

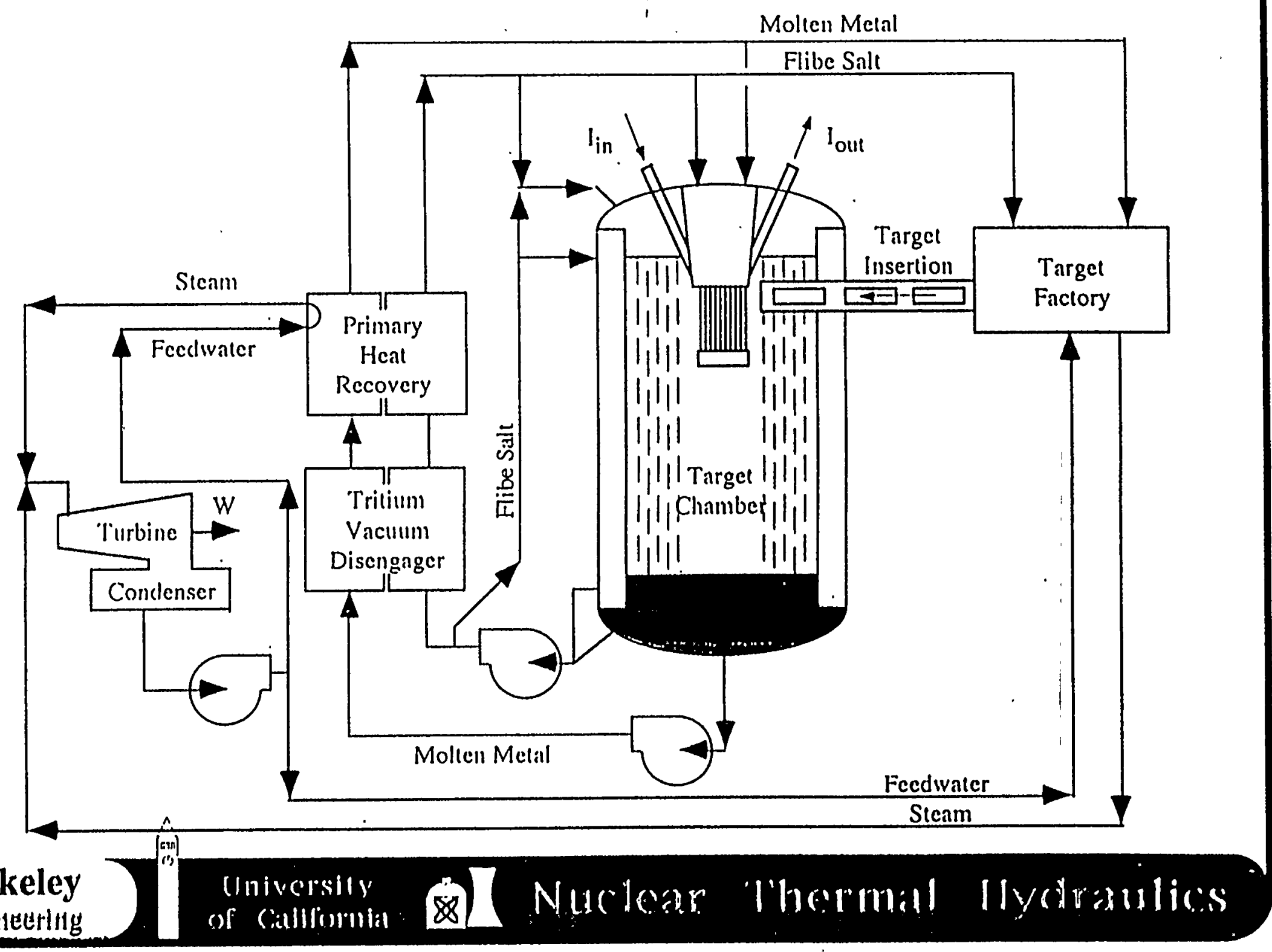




\section{Binary Coolant Summary}

- Tin, Lead and Bismuth are immiscible and liquid in contact with molten Flibe

- Tin appears a good candidate metal:

- Has lowest electrical resistivity

- Density substantially greater than Flibe, allowing easy mechanical separation and recovery

- If inventory can be kept low, isotopic tailoring may reduce activation potential, however remote electrode fabrication will certainly be required.

- Niobium and Molybdenum appear to be good candidate materials for solid electrode interface

- Refractory, low solubility in tin

- Electrical resistivity comparable to solid tin 


\section{Casting Binary Electrodes}

- Flibe has higher freezing temperature $459^{\circ} \mathrm{C}$ and thus is cast first.

- Tin $\left(230^{\circ} \mathrm{C}\right)$ (or lead/bismuth) are cast second

- Spray films onto Flibe substrates

- Cast in penetrations in Flibe

- Cast and machine separate metal components and weld together

- Cast Flibe mechanical properties are not yet know, likely to have good compressive strength, poor tensile strength.

- If tin is frozen as films or cast in penetrations in Flibe, it will solidify at a higher temperature than Flibe

- Upon reaching uniform temperature, tin goes into tension creating structures similar to prestressed reinforced concrete.

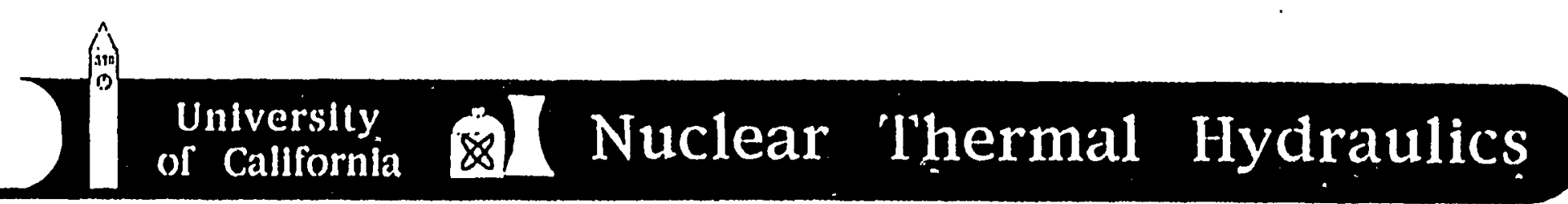




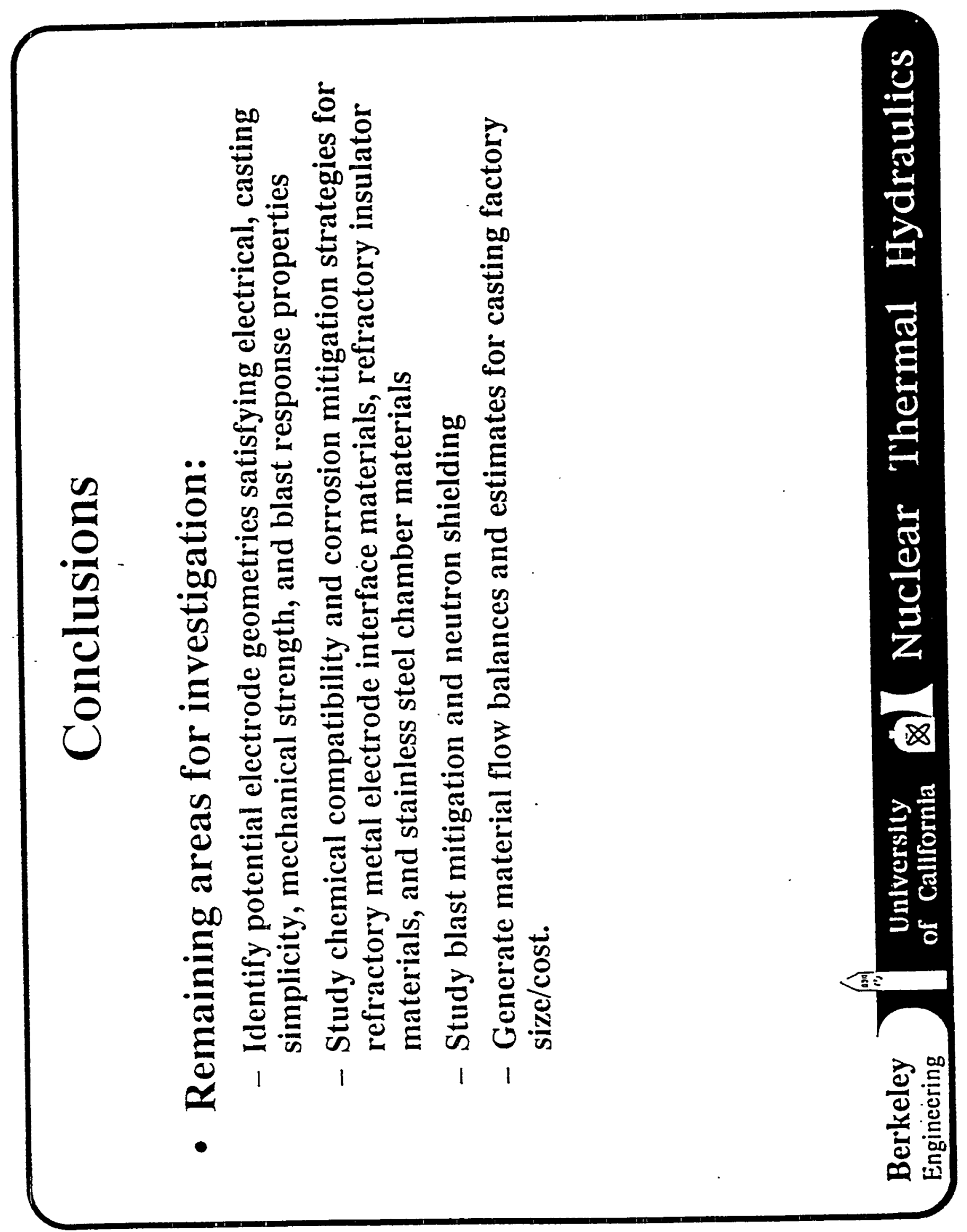




\section{Ion Beams to Repetitively Drive IFE Z-Pinch Targets}

R.R. Peterson, G.L. Kulcinski, D.C. Kammer,

H.Y Khater, E.A. Mogahed,

G. Rochau, J.F. Santarius, M.E. Sawan,

I.N. Sviatoslavsky, T. Utschig

Z-Pinch IFE Workshop

Sandia National Laboratories

Albuquerque, NM

April 27 - 28, 1999 


\section{Many Options for Powering Z-pinches for IFE; Mass Added to Chamber is One Metric}

Particle beams

Kinetic energy $=>$ induction

Replaceable wires

Pre-formed B / Inverse MHD

Liquid Feeds / Tea Cup

Compact Toroid Injection

Laser-induced Currents

Replaceable Solid Feeds
Low mass

Large mass, momentum

Medium mass

Low mass

Large mass

Low mass

Low mass

Large mass 


\section{Intense Ion Beams Can Carry Mega-Amps of Electrical Current to a Target}

-Ions formed with rep-rated extraction applied-B diodes.

-Large radius channels (laserguided pre-formed discharge) transport ions.

-Electrons either injected or pulled from heated gas.

\section{Pulsed Power}

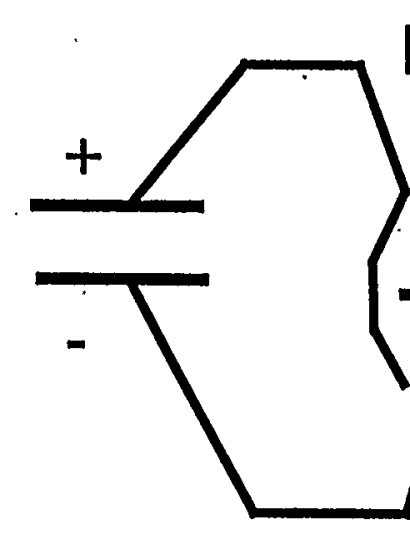

Electrode or Inverse
Diode Diode
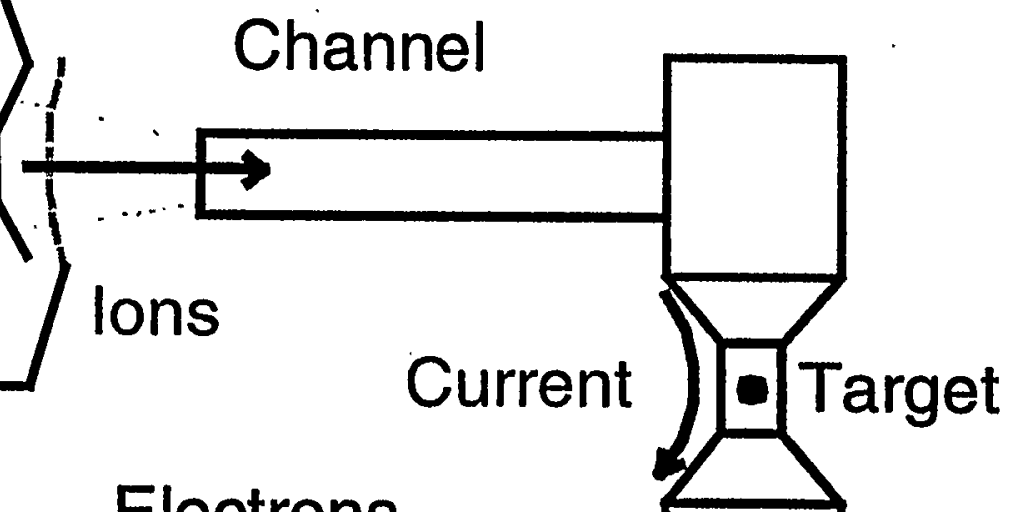

Electrons

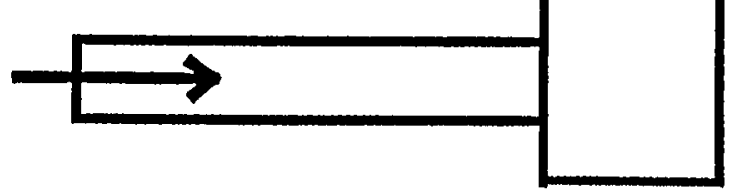

Not to Scale 
Intense Ion Beams for Supplying Current to a Z-Pinch Target Avoid Some of the Issues Faced in Light Ion Fusion

\section{Advantages}

-Larger spots relax the micro-divergence constraints.

-Using Deuterons to carry high currents avoids beam cleanliness and parasitic ion issues.

-Larger spots avoid transport channel expansion and stability issues.

\section{Issues}

-System Efficiency.

-Diode and Pulsed Power Re-rate (Scale up RHEPP)

-Target Coupling and Power Flow in Target 


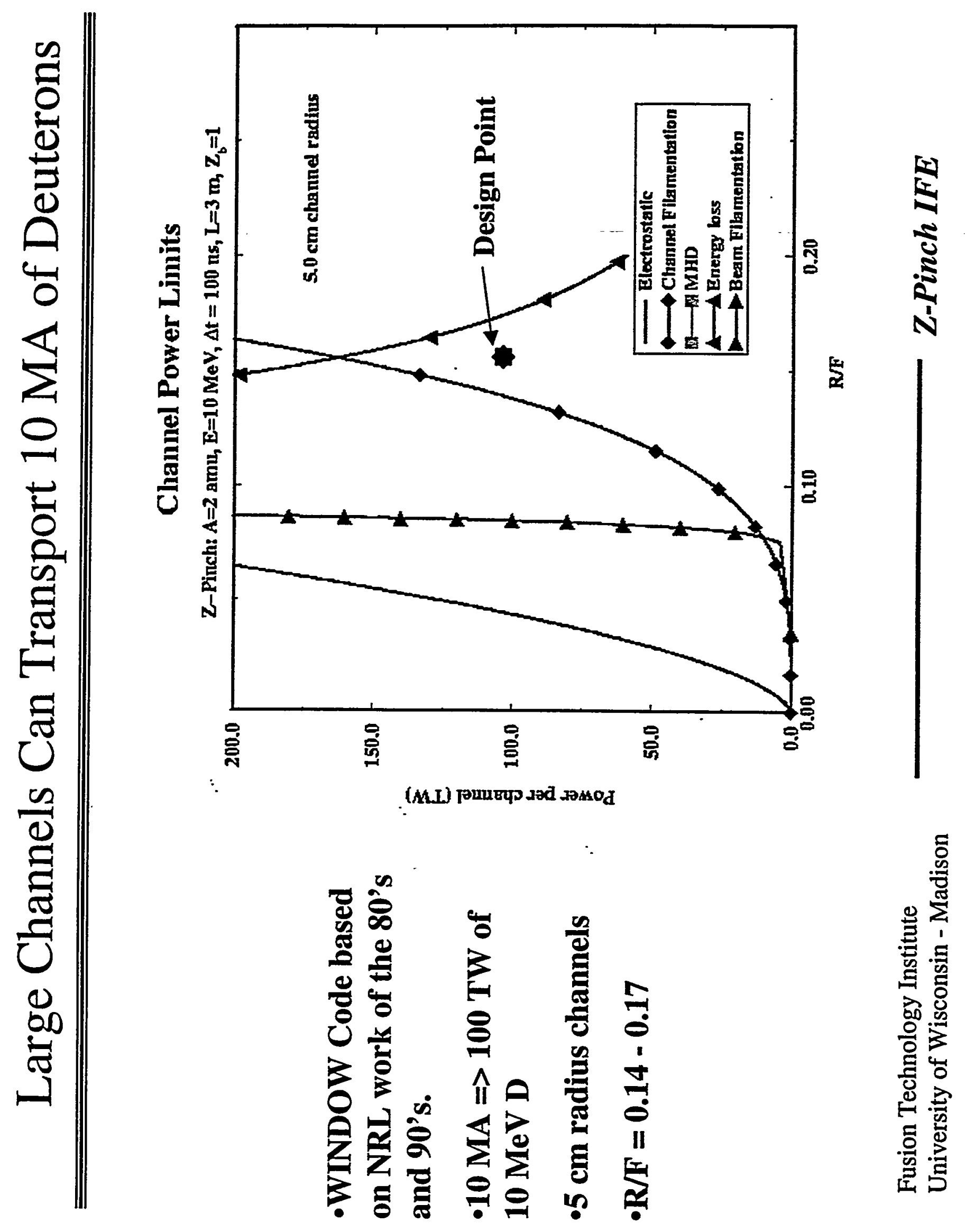




\section{Deuterium Beam Parameters for Z-Pinch IFE}

- MATHCAD Program

Calculates lon Beam

Parameters as functions of Diode Voltage.

- $10 \mathrm{MA}$ of Deutrium ions per Beam (60 MA Total)

- Anode Current Density is Space-Charge Limited with a factor of 5 enhancement.

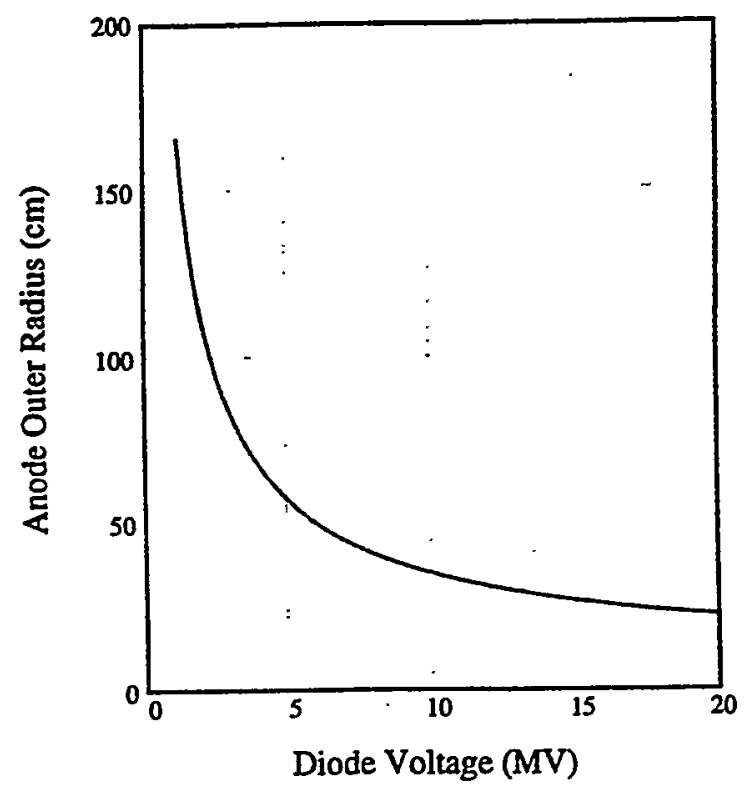

- Hollow Anode with $10 \mathrm{~cm}$ Inner Radius.

- 20 mrad Microdivergence.

- $5 \mathrm{~cm}$ Spot Radius.

- At $10 \mathrm{MV}$, Anode Outer Radius $=35 \mathrm{~cm}$ and $\mathrm{R} / \mathrm{F}=0.14$.

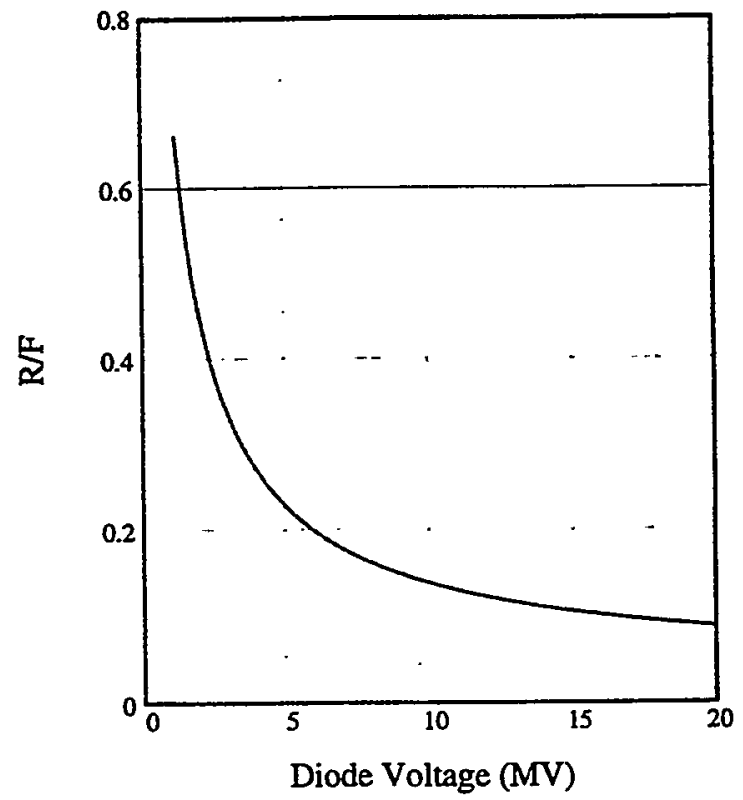

Fusion Technology Institute 


\section{Electrical Parameters are Within Reach of Light Ion Technology}

\begin{tabular}{|c|c|c|c|}
\hline & LIBRA & LIBRA-SP & Z-Pinch IFE \\
\hline Ion Species & Lithium & Lithium & Deute \\
\hline Pulse Width & $9 \mathrm{~ns}$ & $20 \mathrm{~ns}$ & $x_{1}$ \\
\hline $\begin{array}{l}\text { Transport } \\
\text { Length }\end{array}$ & $6 \mathrm{~m}$ & $8 \mathrm{~m}$ & \\
\hline Ion Energy & $22.5 \mathrm{MeV}$ & $30 \mathrm{MeV}$ & $10 \mathrm{NeV}$ \\
\hline $\begin{array}{l}\text { Peak } \\
\text { Current }\end{array}$ & $\begin{array}{c}1.1 \mathrm{MA} \text { (per } \\
\text { beam) }\end{array}$ & $\begin{array}{c}0.46 \mathrm{MA} \text { (per } \\
\text { beam) }\end{array}$ & 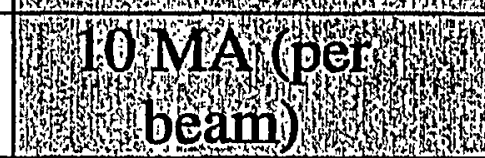 \\
\hline $\begin{array}{l}\text { Micro- } \\
\text { Divergence }\end{array}$ & $5 \mathrm{mrad}$ & $4 \mathrm{mrad}$ & \\
\hline Spot Radius & $0.35 \mathrm{~cm}$ & $0.6 \mathrm{~cm}$ & \\
\hline
\end{tabular}

Fusion Technology Institute

University of Wisconsin - Madison 


\section{Z-Pinch IFE Target Structural and Electrical Requirements}

\section{Target options}

-Dynamic Hohlraum ( capsule inside a wire array)

-Z-Pinch Driven Hohlraum (wire arrays on either side of a Hohlraum)

-Static Hohlraum (wire arrays on either side of a Hohlraum)

\section{Z-Pinch IFE target needs to:}

-Couple to power source with the proper electrical impedance.

-Survive target injection (structurally and thermally).

-Create a minimum mass of radioactive debris.

-Provide a yield $/ \$$ of fabricated cost $>3000 \mathrm{MJ} / \$(\sim 3 \mathrm{mils} / \mathrm{kWhr}$ to $\mathrm{COE}$ ). 


\section{A Dynamic Hohlraum Target with Low Mass Electrodes to Collect Charge is One Option}

A static Hohlraum design should also be considered.

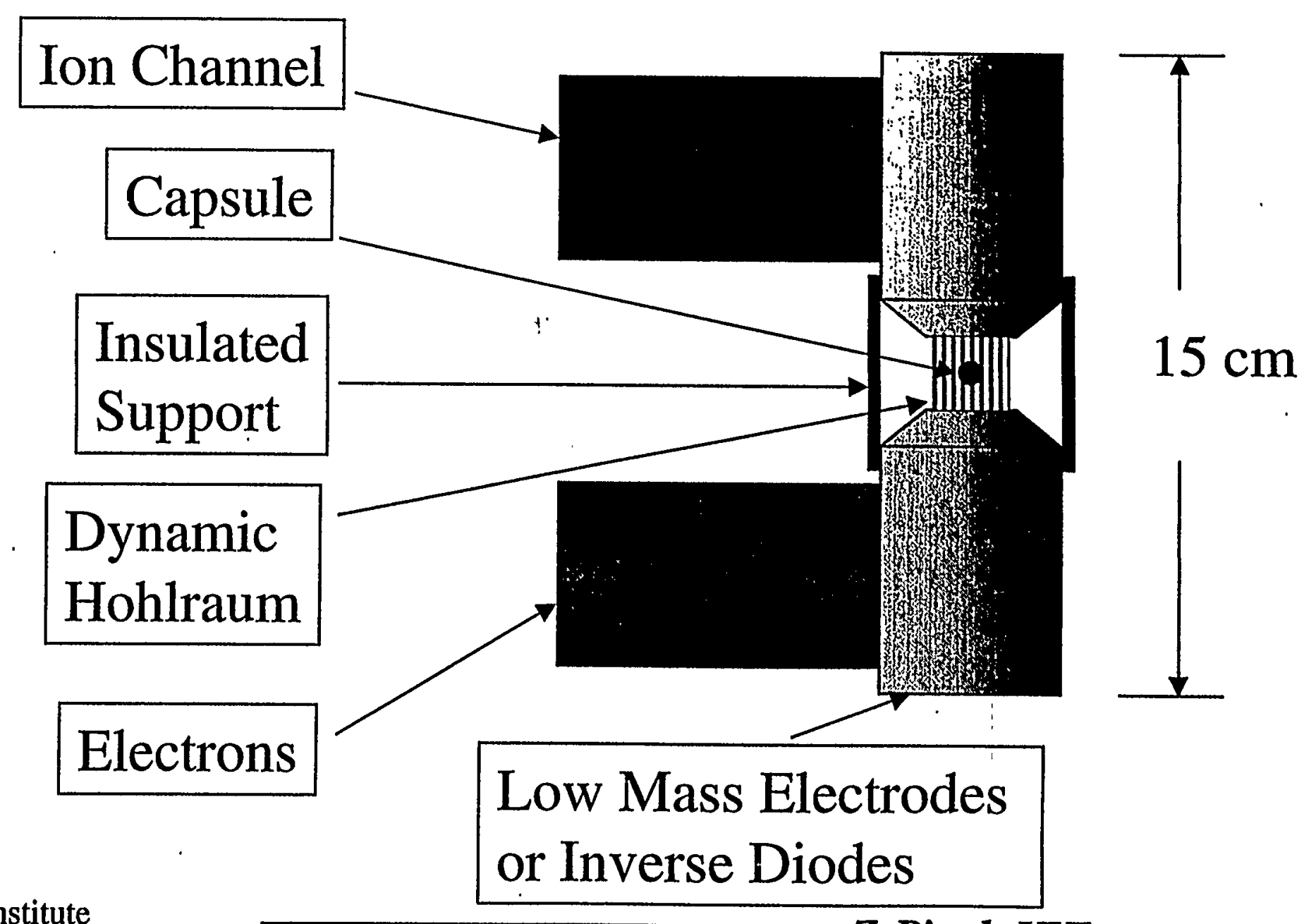

Fusion Technology Institute

University of Wisconsin - Madison

Z-Pinch IFE 


\section{Z-pinch-driven hohlraum future plans}

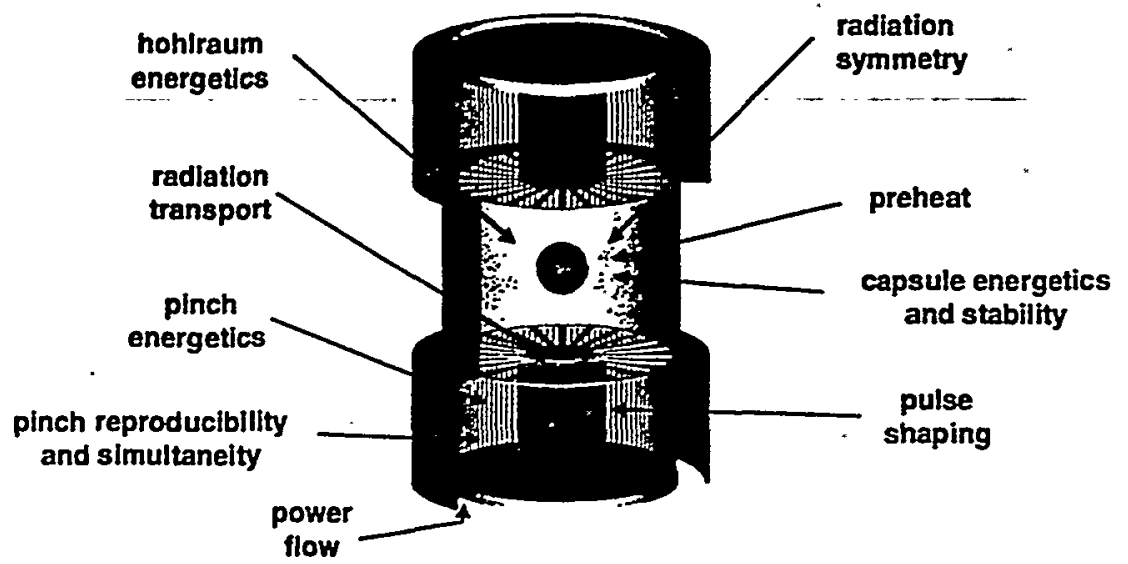

fi. J. Ponter, M. Cuneo, D. L. Hanson, A. Spielman,

(17) R. Vosey, $L$ Ruggles W. Simpson

ICF Target Design Workshop

J. Hammer. N. Landen, P. Rambo

May 5-6, 1999 -

\section{There is much work to do to qualify}

this approach for high-yield

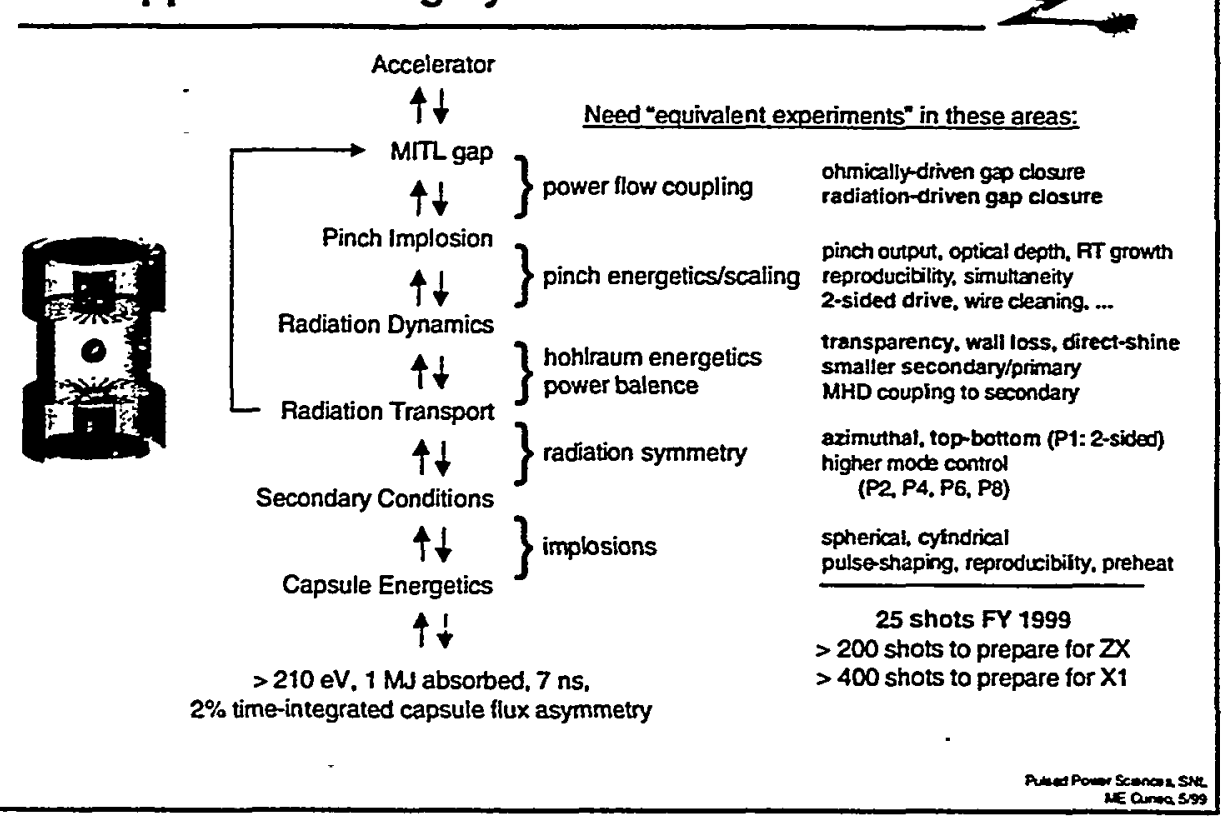




\section{Future $Z$ experiments will baseline critical scaling issues, study symmetry and implosions}

Power flow, pinch coupling experiments, pinch energetics (1st shots June)

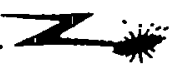

- measure B-tamped radiation-driven-gap closure at several B's, gaps, and Ts. Dower coupling to pinch as a function of AK gap gap closuze tor energy baknce assessment

1-d geometry - physes at cross freld transport

- multiple plasma densities and techniques:

$1013 \mathrm{~cm}-3$

plasma that carnes electric field and shunts aurrent emussion and absorption spectroscopy w/seeding? (Bailey)

1015 - $1018 \mathrm{~cm} 3$ plasma that carties shurts currem

spectroscopy. Laser dagnosics

$>1 e 21 \mathrm{~cm} 3$ materal that corfined radiation fietd in hohraum

wall ro-emission backightng or laser backighting

- power flow and pinch repeatibility issues

use hohlraum as an iregrating sphere to most acourately measure pinch power unproved current measuremems (power coupling through comvolute and to pinch) plasma formation in convolute and teed gap
$E \propto t^{2} \propto T^{36}$
$P_{\text {mag }} \propto B^{2} \propto F^{2} / R_{m}^{2}$
$P_{\text {abi_ }} A_{u} \propto T^{2.6}$
$P_{\text {col_lowz }}=T^{3.5}$
$\frac{P_{\text {mag }}}{P_{\text {sbi_Au }}} \propto T / R_{\text {mino }}^{2}$
$\frac{P_{\operatorname{mog}}}{P_{\text {mod }} \times T^{0.1} / R_{\text {mre }}^{2}}$
B-tamping improves with increasing temperaturel!
$T 100-150 \mathrm{eV}, R_{m}=1$ to $2 \mathrm{~cm}$
Ratio of T/F? can vary by a factor of 10

Are $Z$ expenments (20 MA. $150 \mathrm{eV}$ ) an oventest of this criterion? or do we need to test this at full $50 \mathrm{MA}$. an $0210-240 \mathrm{eV}$ levels in a primary?

\section{Future $Z$ experiments will baseline critical scaling issues, study symmetry and implosions}
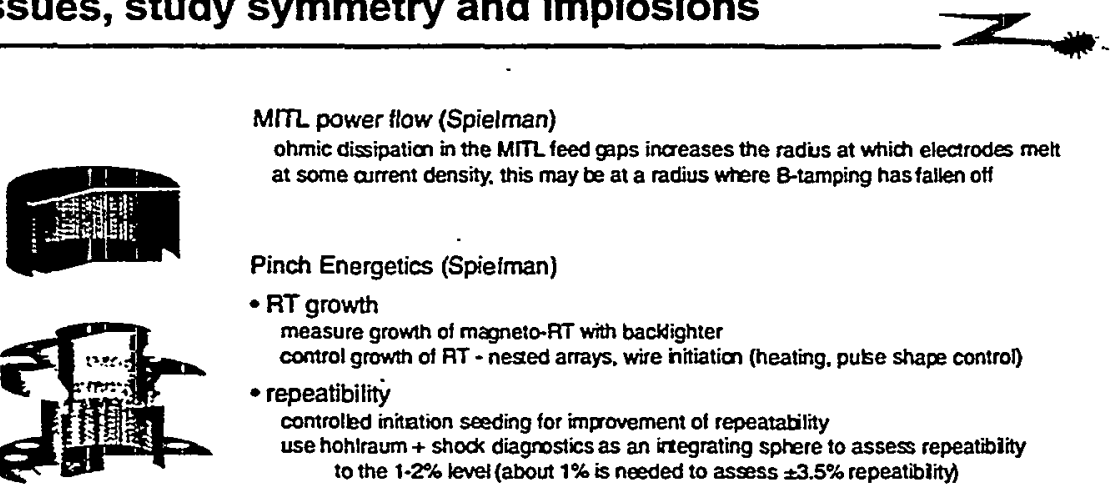

MIn power flow (Spielman)

ohmic dissipation in the MITL feed gaps increases the radius at which electrodes meth

at some current density, this may be at a radius wtere 8-tamping has fallen off

Pinch Energetics (Spieiman)

- RT growth

measure growth of magneto-fT with backlighter

control growth of RT - nesed arrays, wire intiation (heating, putse shape controi)

- repeatibility

controled intiation seeding for inprovement of repeatability

use hohlraum + shook diagnostics as an irtegrating sphere to assess repeatibitity

to the $1.2 \%$ level (about $1 \%$ is needed to assess $\pm 3.5 \%$ repeatibility)

- pinch opacity experiments (scalability of $x$-ray power $\left.\alpha\right|^{2}$ )

scale pinch opacny at constast implosion time by decreasng r. opacity $\alpha$ mass $\alpha 1 / r^{2}$

or increase imploson time (opacity $\alpha$ mass $\alpha t^{2}$ )

different punch materials to decrease opaciy

Can $Z$ experiments with smaller radus and longer pulselength test opactly mitugation techniques that scale to a 55 MA pinch' Do we need another facility to test at the full tevel? 


\section{Pinch reproducibility and simultaneity are critical to control P1 in two-sided systems}

10 independent shots, nominally identical setups, data courtesy WAS/ABS
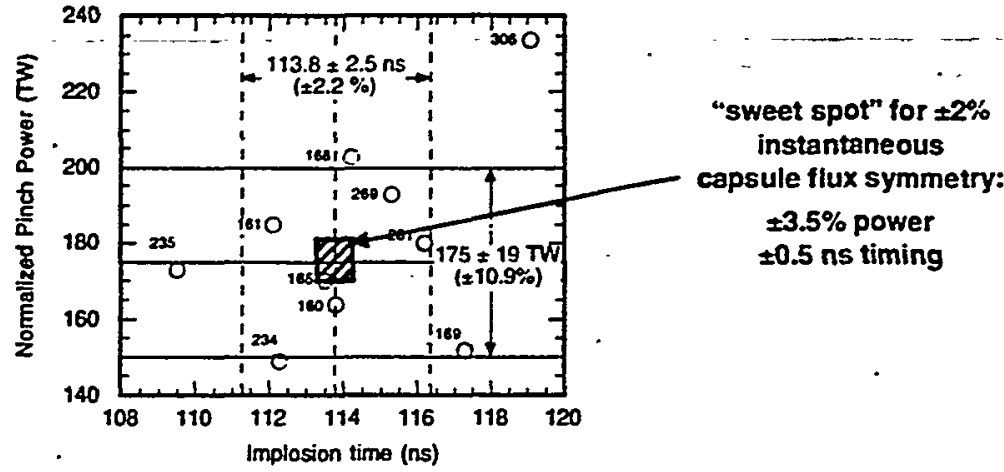

- Shot-10-shot spread in power $( \pm 11 \%)$ is less than error in power measurement $( \pm 15 \%)$

- Improved instruments, data acquisitlon, experimental method and variable control is necessary

- Wall motion, time-averaging, and optimization techniques may increase size of sweet spot

- Other techniques may decrease sensitivity to offsets

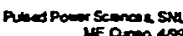

\section{Future $Z$ experiments will baseline critical scaling issues, study symmetry and implosions}

Develop 2-sided drive for $Z$ (shots in June and 4th quarter)

- single feed, 2-sided feasibility.

power flow (nductance problems), pinch dyramics, secondary diagnosiblity, seconctary flexbility, energetics penalty

- assess reproducibility, uniformity

there is only 1 comoute and 1 radial gap - improves repeatibliy?

there is an extended, radiation driven gap between 1st and and pinch

apertures could perturb upper pinch

ditferent pobsty of pinch to secondary side

wre array construction techniques are difficat

- if possible

we improve P1 for implosion experiments.

then use capulses to monito few percent timing and power imbalences
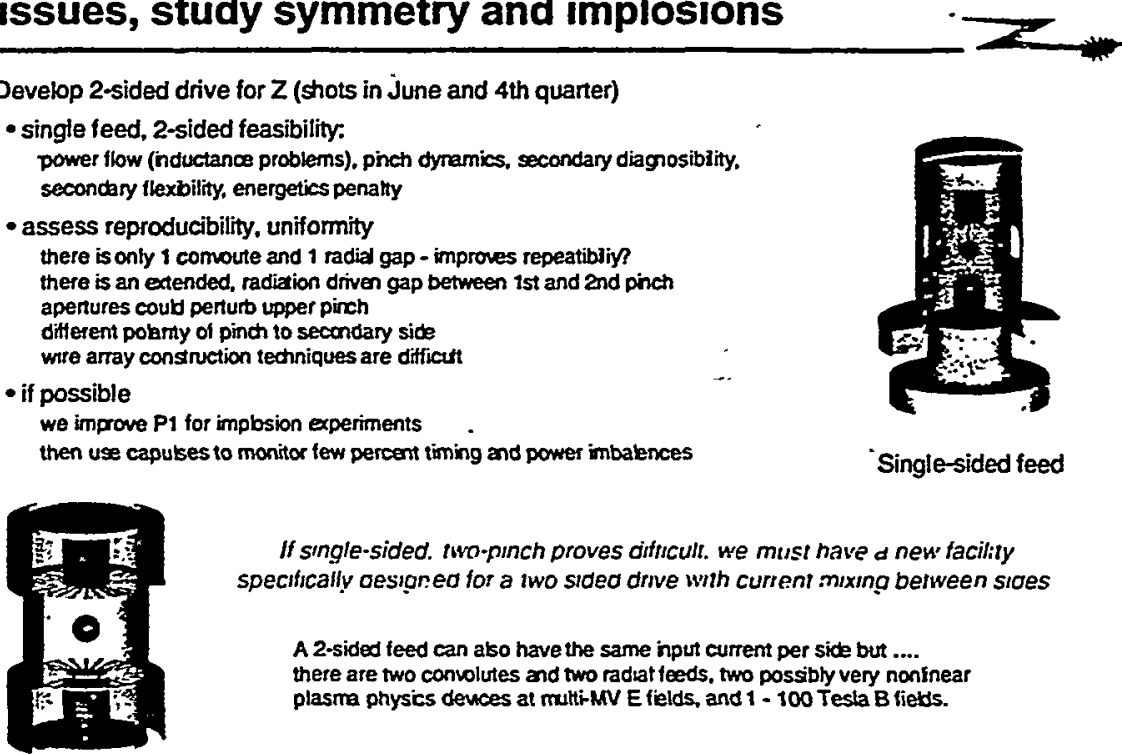

If single-sided. two-punch proves diffucult. we must have d new facility specifically oesioned for a two sideo drive with current mixing between siges

A 2-sided feed can also have the same input current per side but ... there are two convolutes and two radiat feeds, two possibly very noninear plasma physics dences at multi-MV E fields, and $1-100$ Testa B fiebs. 

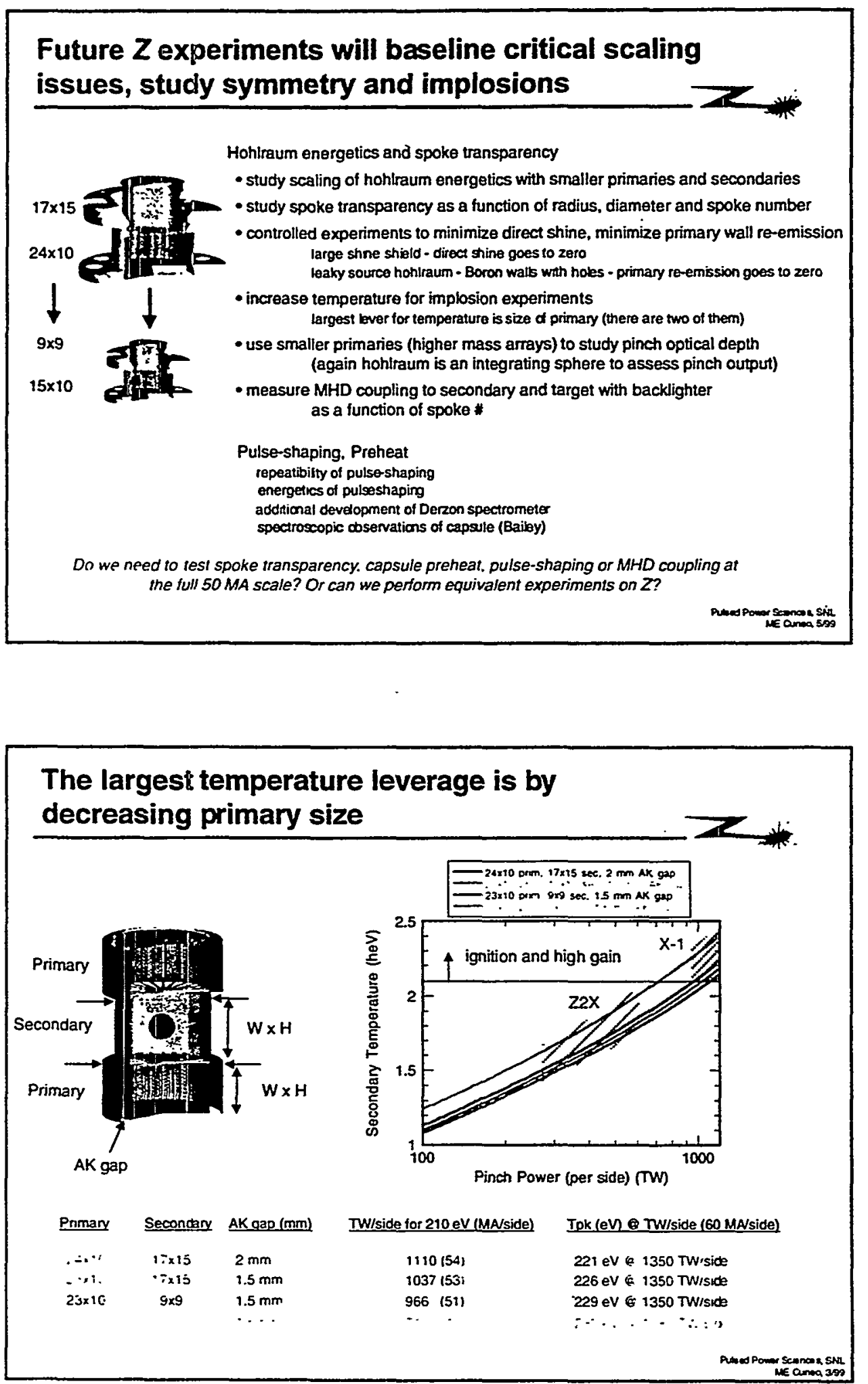

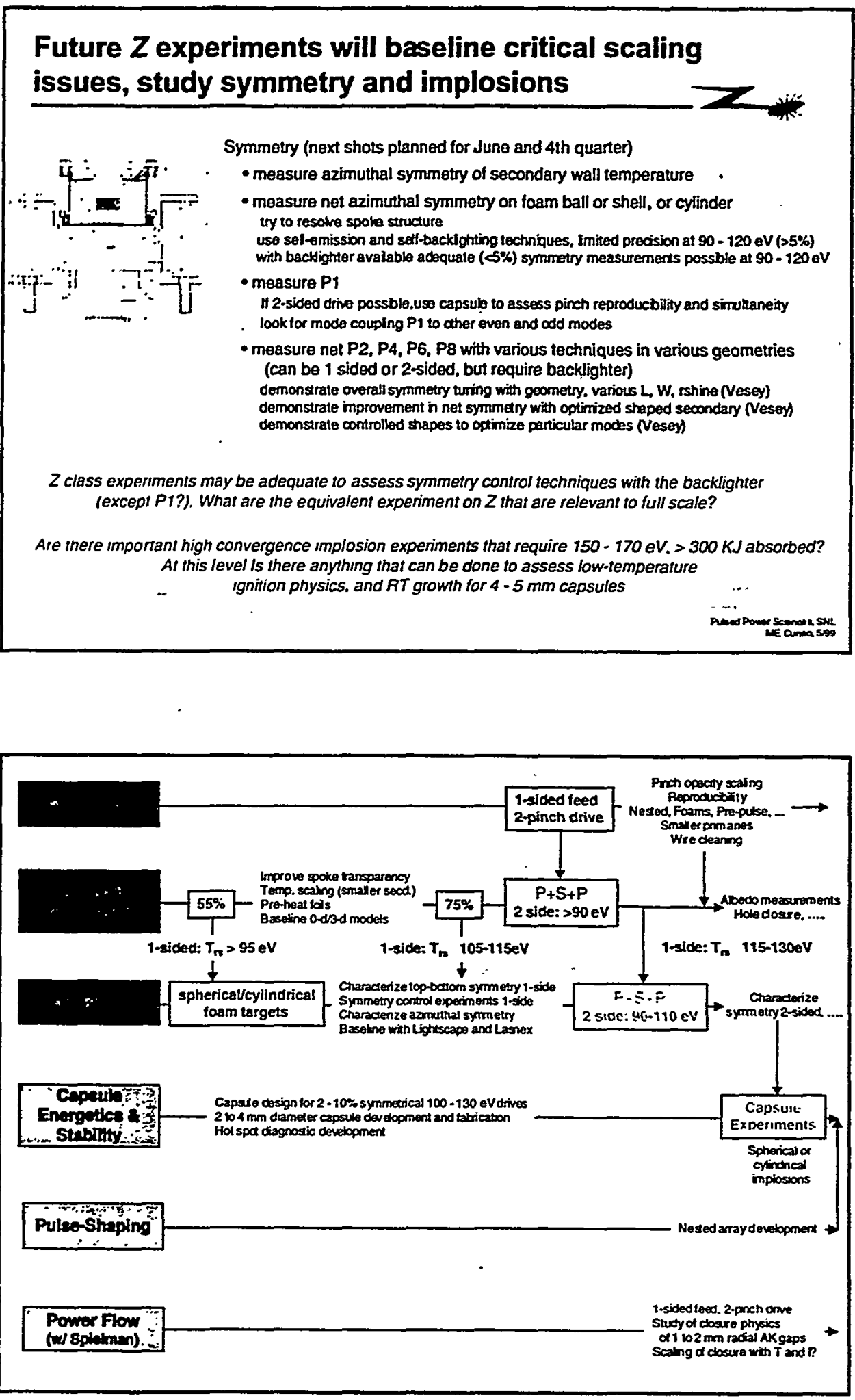


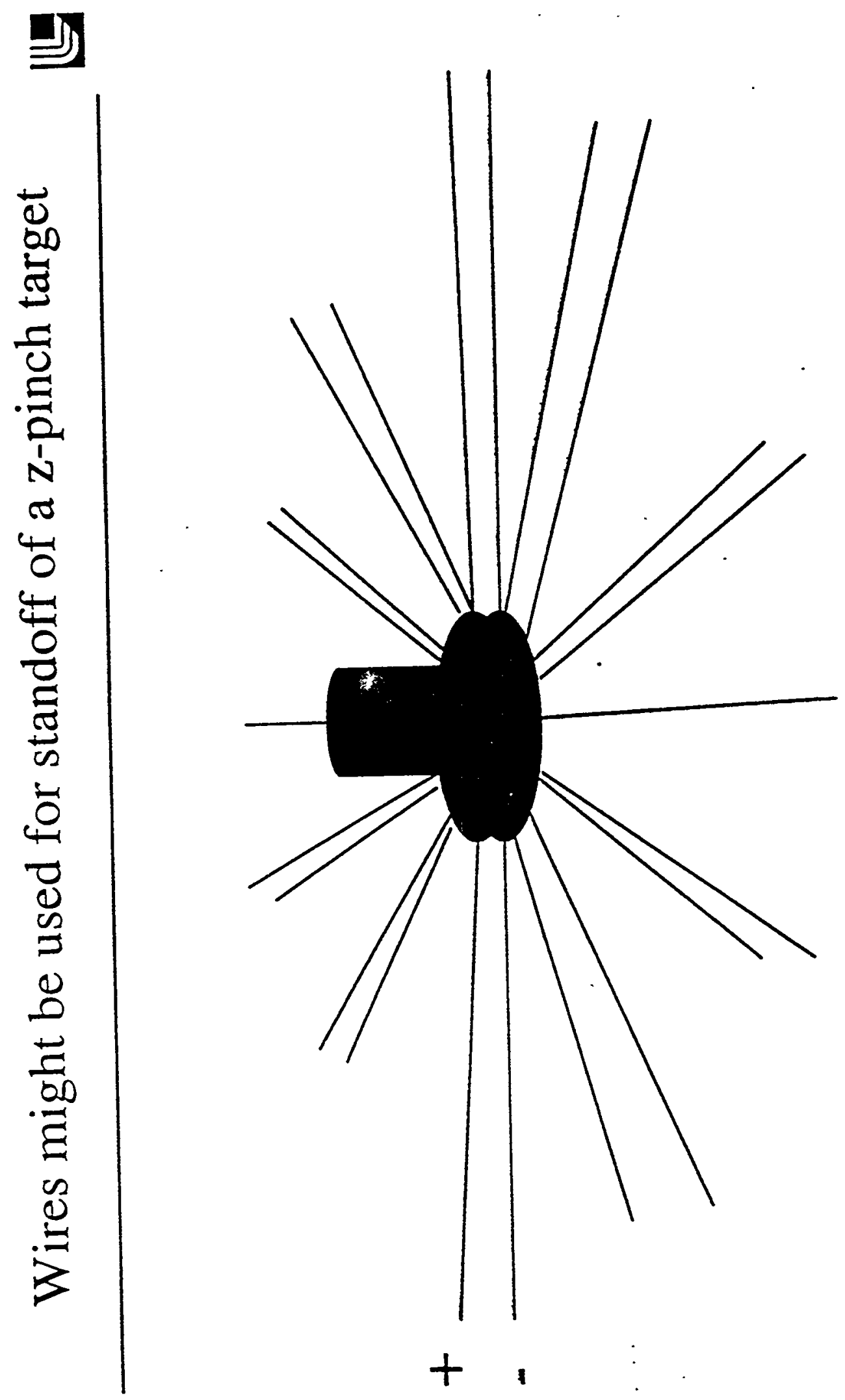


At $<1 \mathrm{hz}$, actuators could connect wires to the target

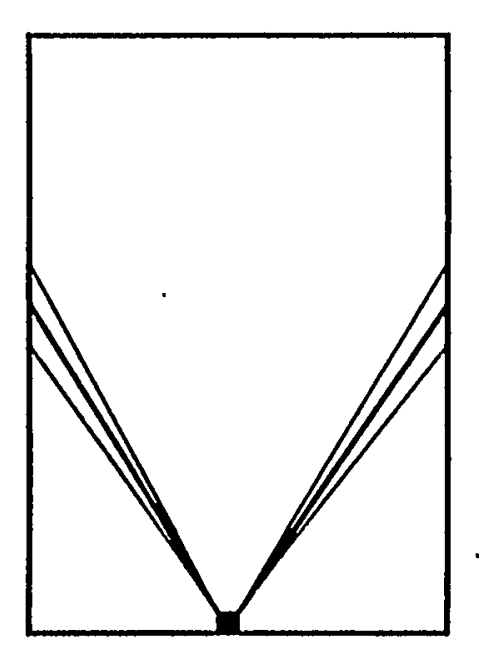

Target

inserted and wires

pulled

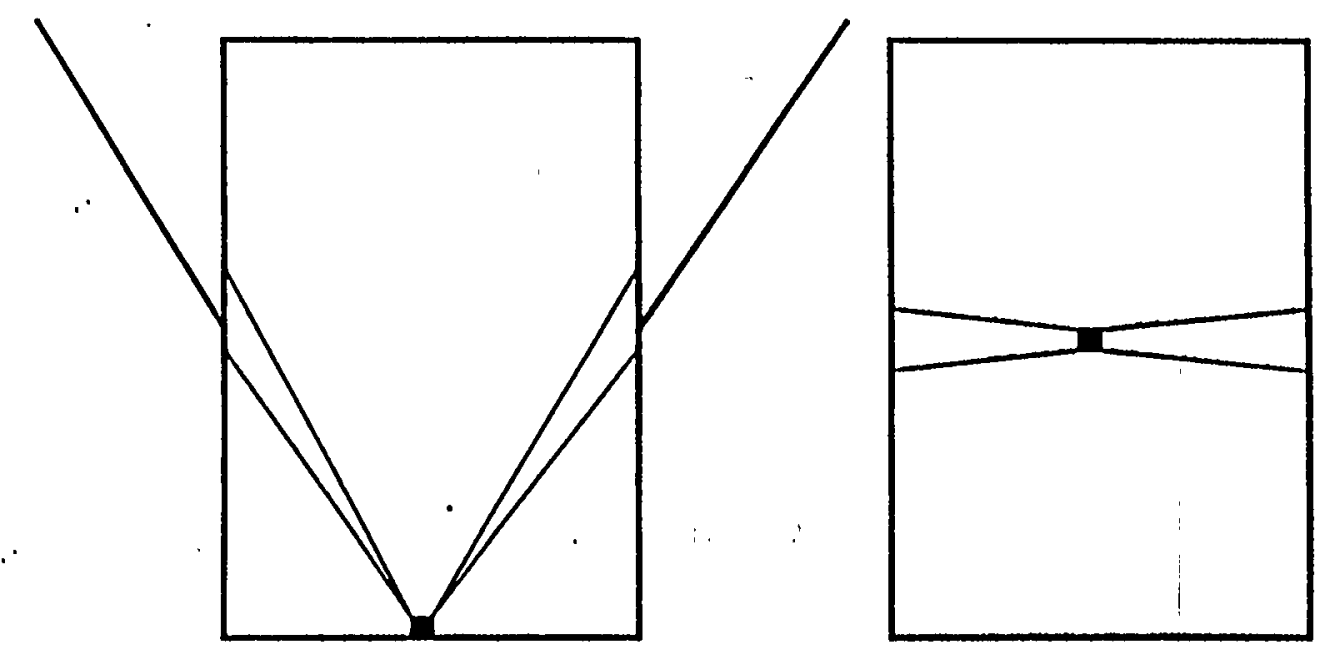

- actuators

withdrawn
Wires

tightened

to position

target 


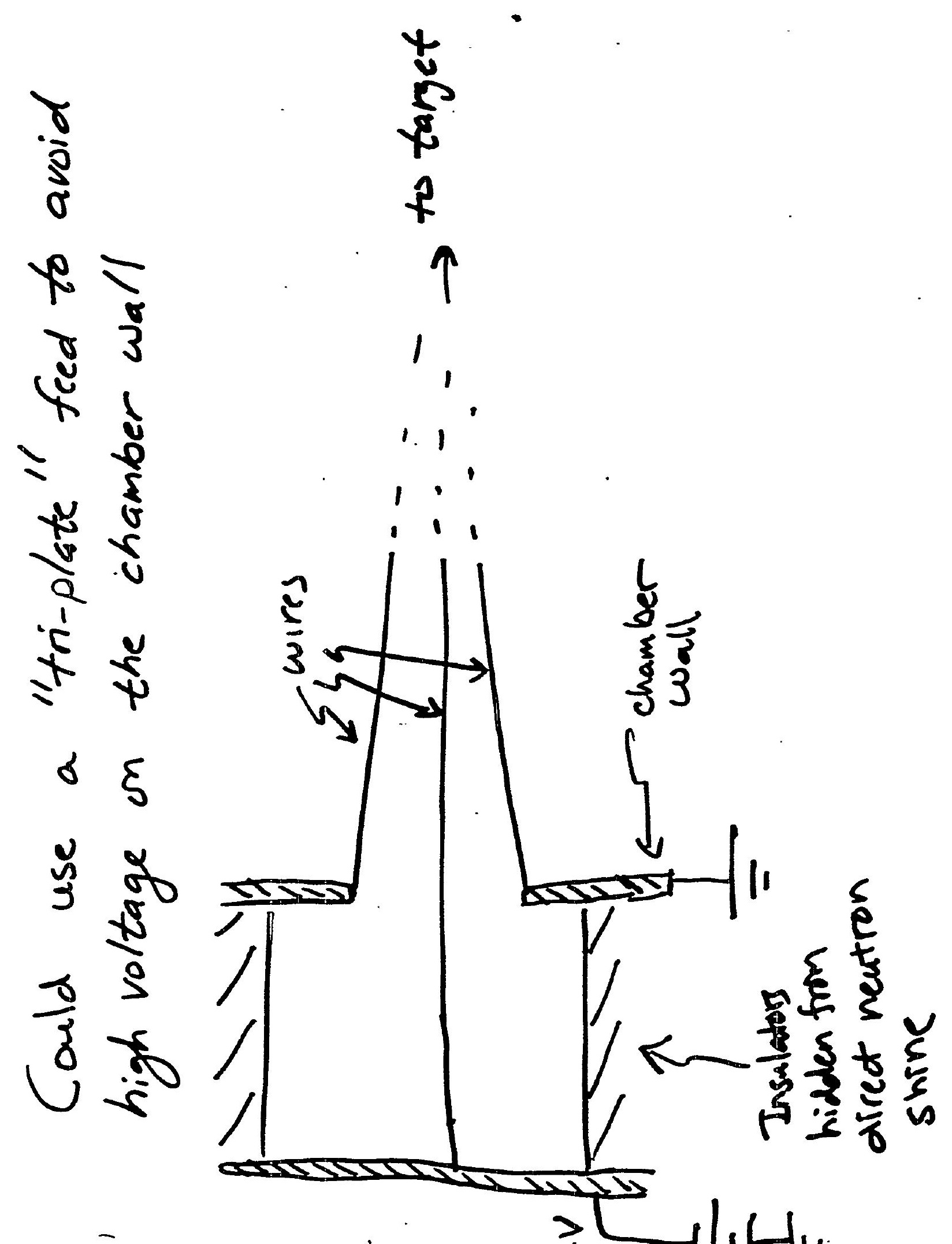




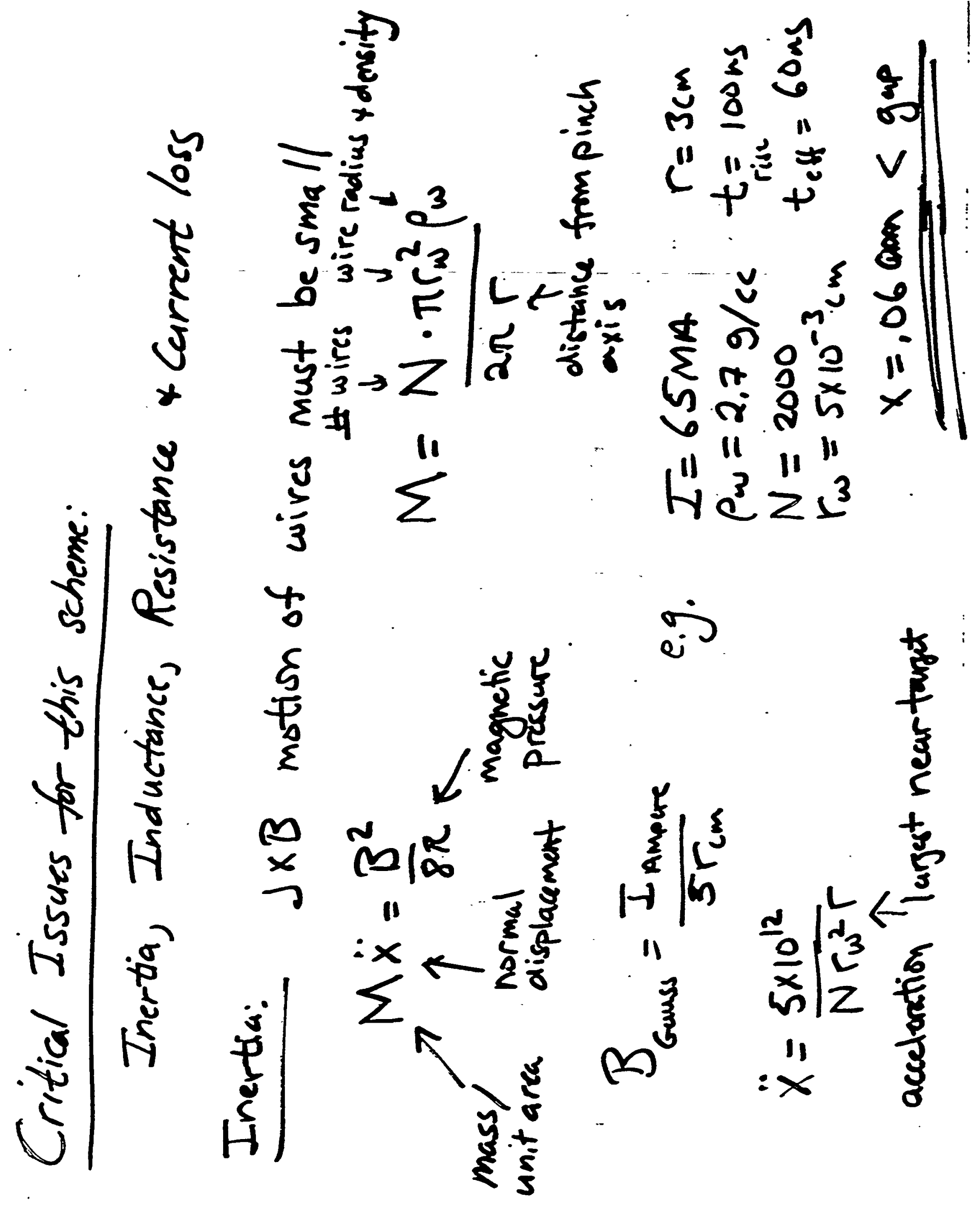




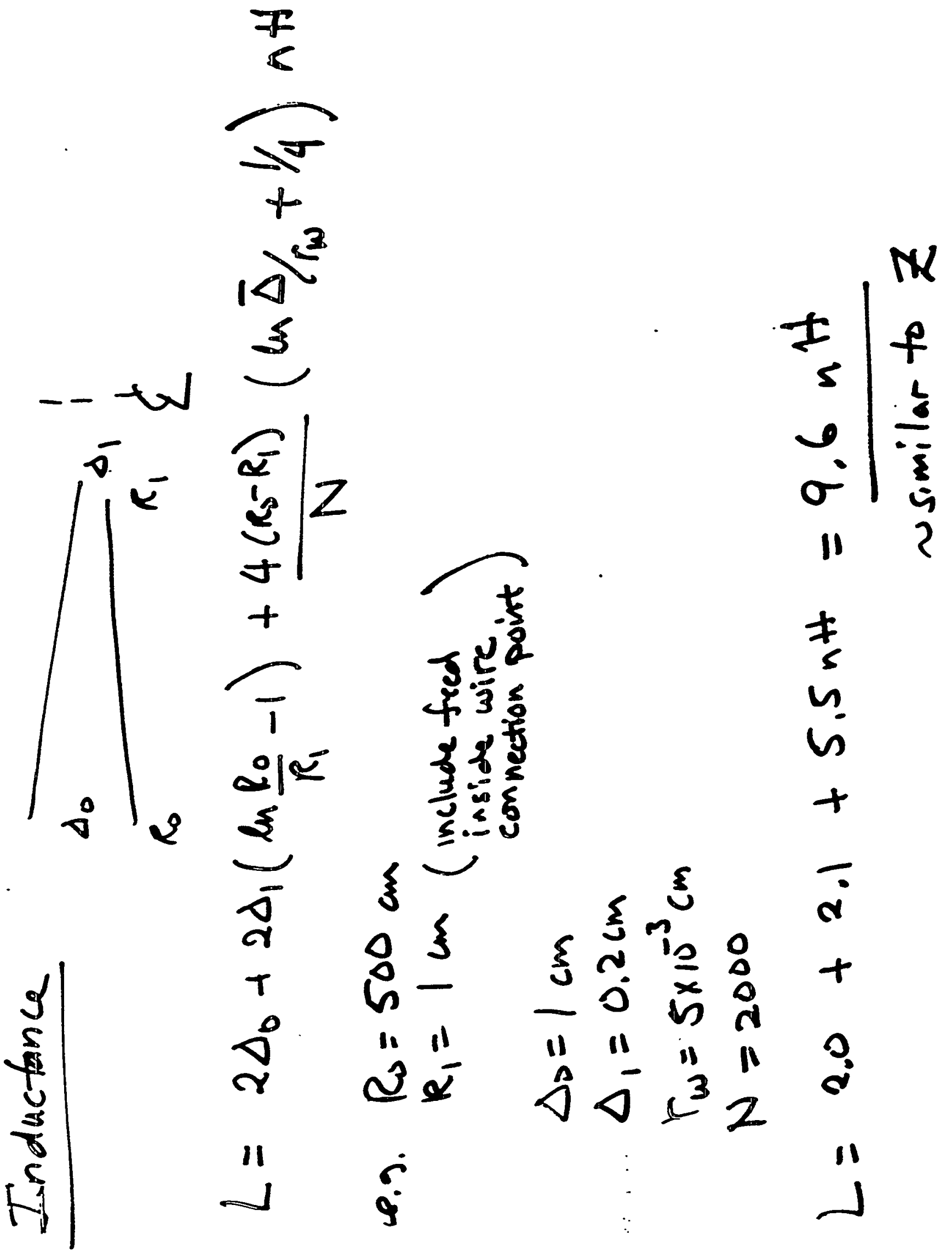




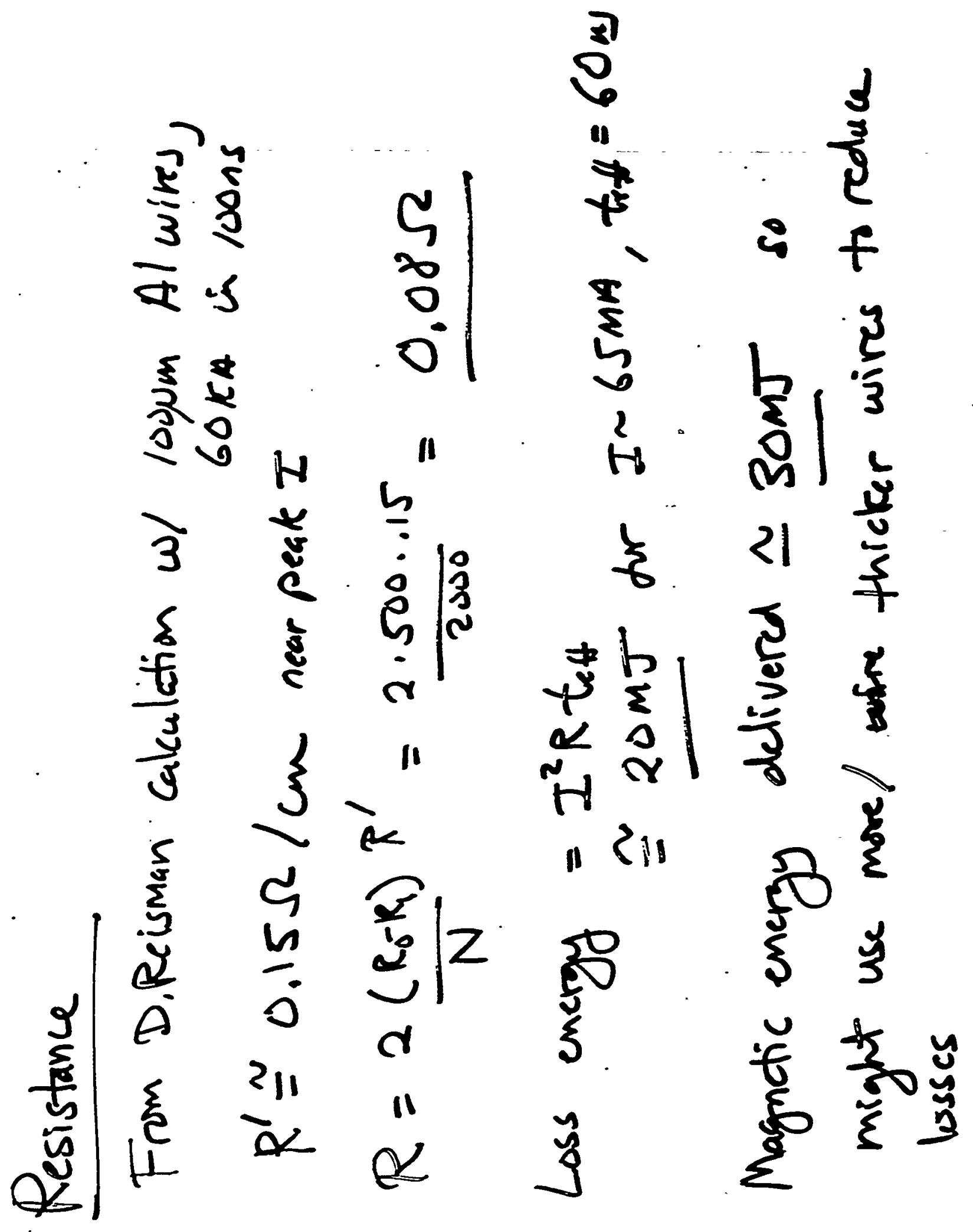




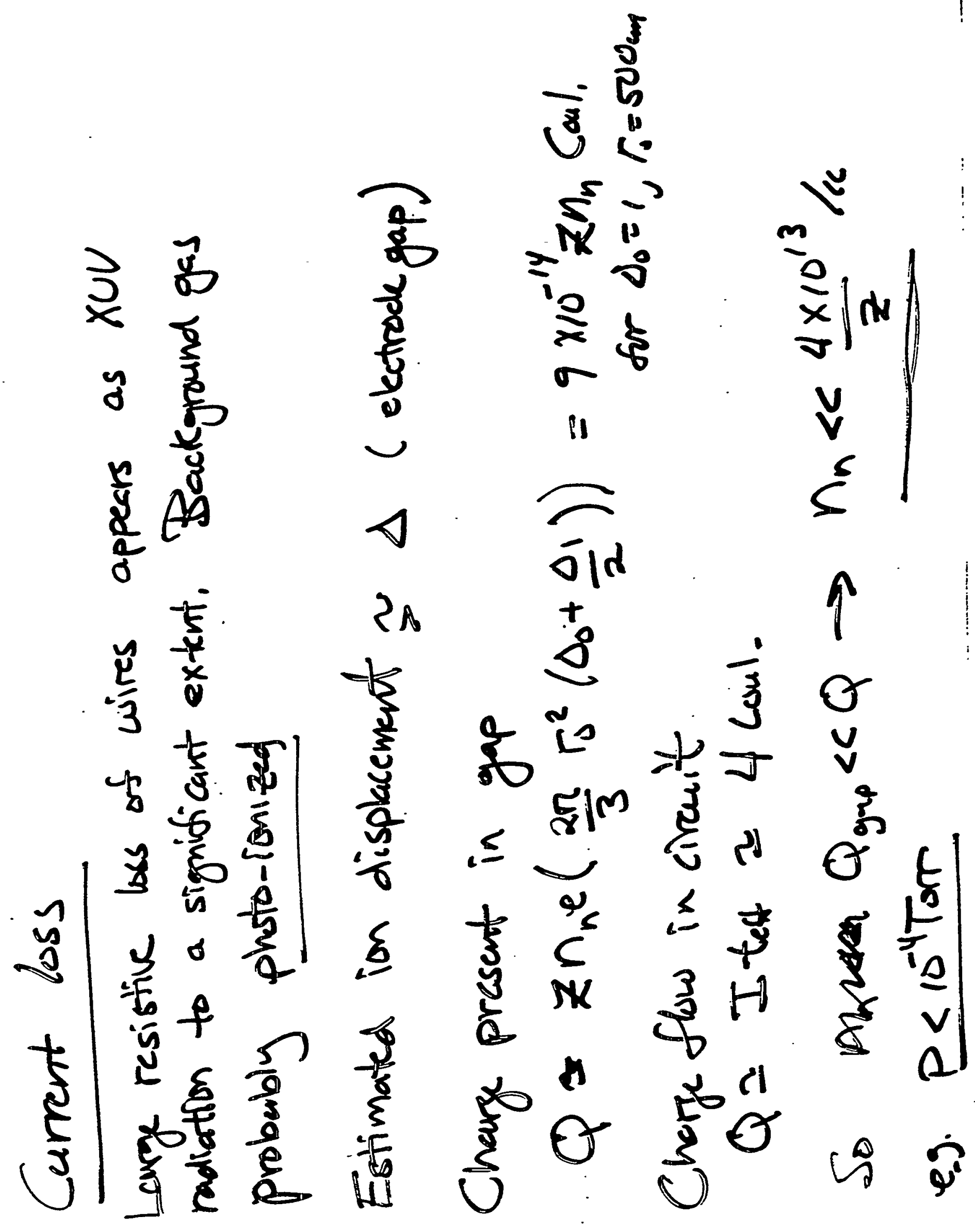




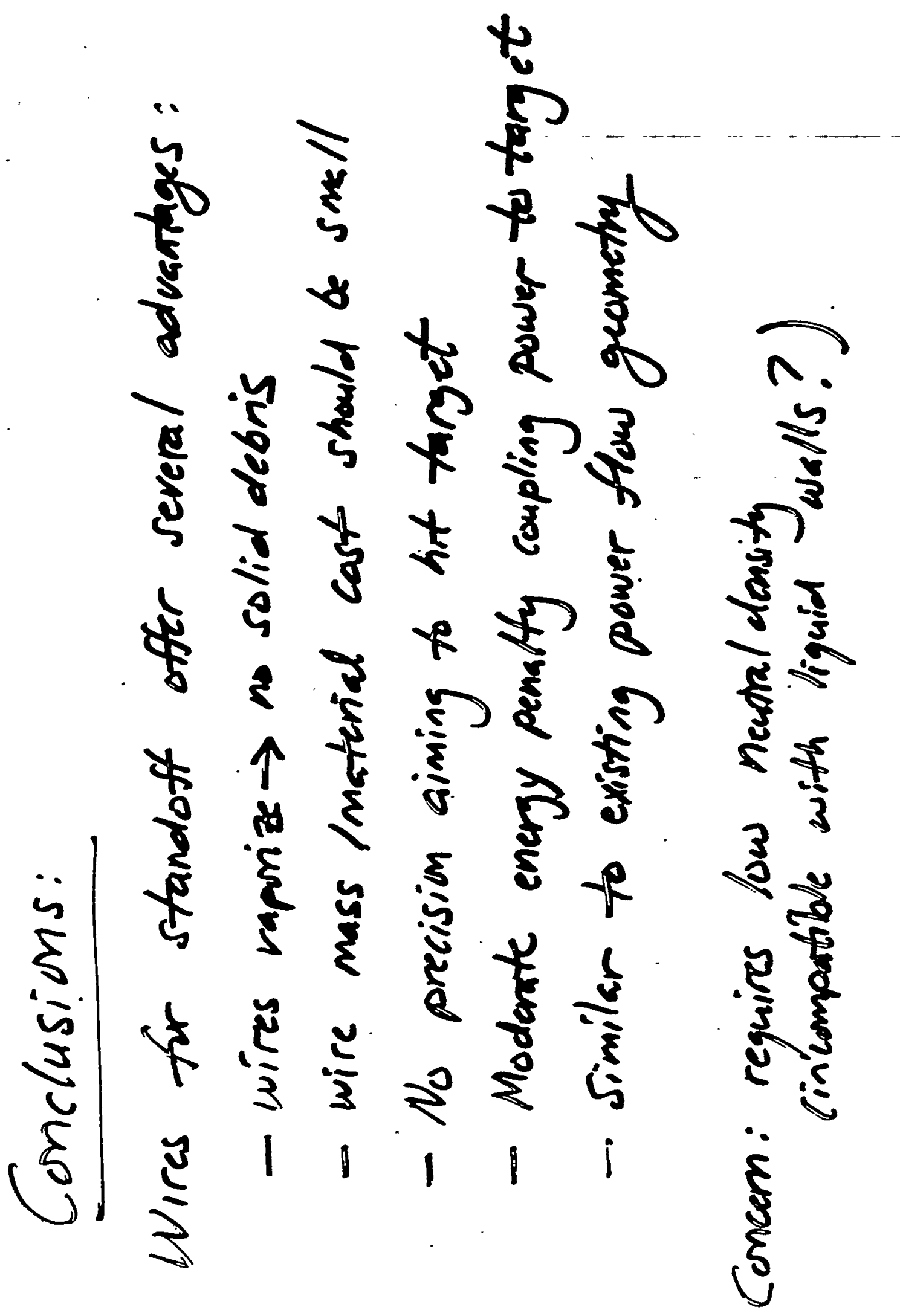


INVERSE ION DIODES FOR DRIVING Z-PINCH IFE

G. Cooperstein, P.F. Ottinger, and D. Mosher

\author{
Pulsed Power Physics Branch \\ Plasma Physies Division \\ Naval Research Laboratory \\ Washington, DC \\ Presented to \\ Z-Pinch IFE Workshop
}

April 27-28, 1999

\title{
Outline
}

- Brief history

- Conceptual overview

- Inverse electron beam diodes

- Inverse ion diodes

- Point design

- Ion source options

- Ion transport options

- Inverse diode options

- Issues 


\section{Brief History}

- SNL electron beam fusion research with wire initiated z-discharge NRL transport (EBFA) - early 70 s

- Rudakov proposed capturing transported electron beams with an inverse electon beam diode and driving a z-pinch - mid 70s

- massive disposable transport channels

- Cornell applied-B ion diode research - early 70 s.

- NRL pinch-reflex ion diode research with protons- mid $70 \mathrm{~s}$ to early $80 \mathrm{~s}$

- focusing, z-discharge transport and final focusing

- SNL LIICF program - mid 70s to mid 90s

- applied-B diodes with heavier ions

- NRL, Cornell and other collaborators

- Recent NRL ion transport research

- low-mass z-discharge, wire-guided, ballistic, and self-pinch transport

- all of above part of SNL's LIICF : or LBNL's HIF program

- Cooperstein suggested ion beam power convergence and inverse ion diodes for PRS loads at DNASNL Full Threat Simulator meeting in 10/92

- Slutz suggested ion beam driven z-pinches for IFE in $2 / 97$ memo

- Melhorn reiterated suggestion at BEAMS'98 (6/98)

\section{Conceptual Overview}

- Produce protons with ten or more self-magnetically insulated ion diodes with pulsed plasma anodes

- also consider applied-B or MAP ion diodes or

- higher- $Z$ ions from two-stage applied-B ion diode with stripping

- Transport ions to large inverse ion diode using ballistic transport

- also consider self-pinch transport or

- transport with laser-initiated Z-discharge channels or

- low-mass wall-confined z-discharge channels and/or

- bunching by factor of 2 or. 3

- Capture ion beams in inverse barrel ion diode with electrons inhibited through self-magnetic insulation, and drive two annular z-pinches surrounding pellet in heylraum

- also consider applied-B barrel ion diode with precharged capacitors 


\section{Advantages}

NRL

- Builds on over 20 years of Light lon Inertial Confinement Fusion

(LIICF) production, focusing and transport research

- Inverse ion diode z-pinch concept has > 10X larger target than LIICF

- relieves divergence constraint

- simplifies ion transport

- allows use of protons and simpler, self-magnetically insulated ion diodes both at source and at target

- Ion beam cool relative to electron beam and can be recaptured with higher efficiency

- Ion bunching could provide current multiplication during transport

- Charge- and current-neutral ion transport may provide easier path to energy convergence than self- magnetically insulated transmission lines (MITLS)

Simple 1-D Child Langmuir Inverse Electron Diode lllustrates Concept (no ions or self-magnetic field effects are included)

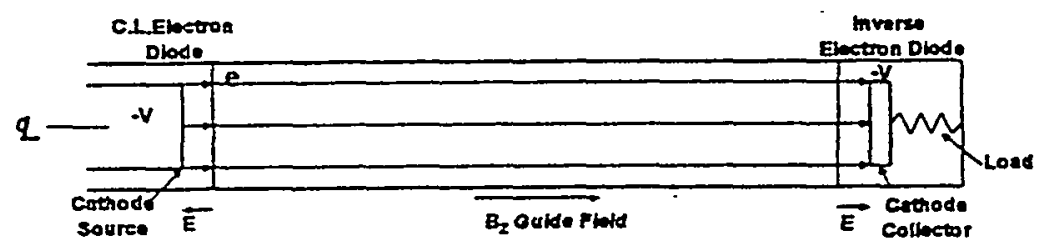

- Electrons accelerate to voltage

- Electrons decelerate from $V$ to $V$ against electric field from zero along electric field and cathode to anode

- $Z_{\text {dode }}=V / I$ soft-land on cathode collector

- $Z_{\text {foed }}=V I I$

- Depressed collector schemes for Gyrotrons are example of successful application of this technique with cool laminar electron beams

- High temperature electron beam has low recapture efficiency

- "heat" cannot be recaptured

- also, losses to ions could be large 


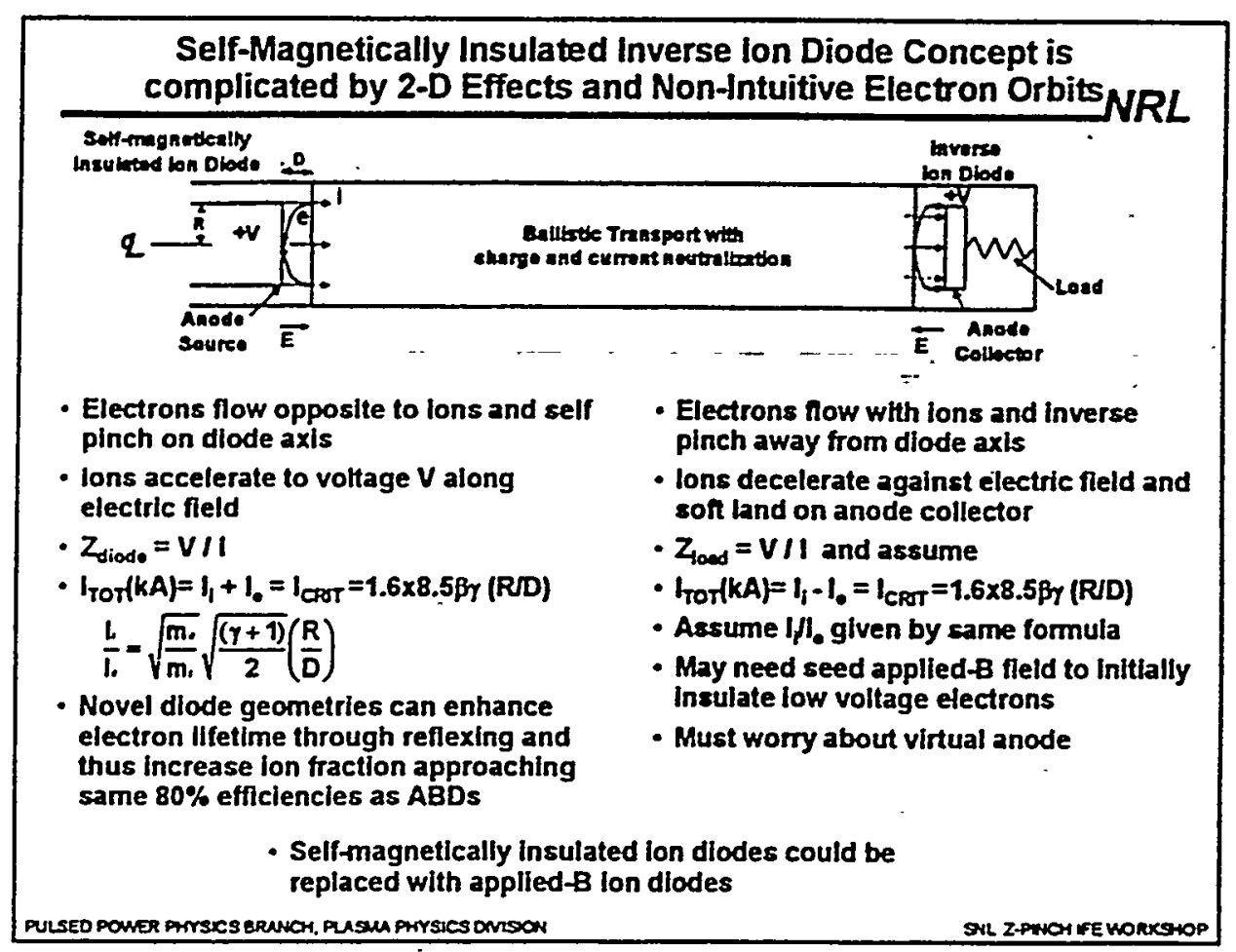

$-$

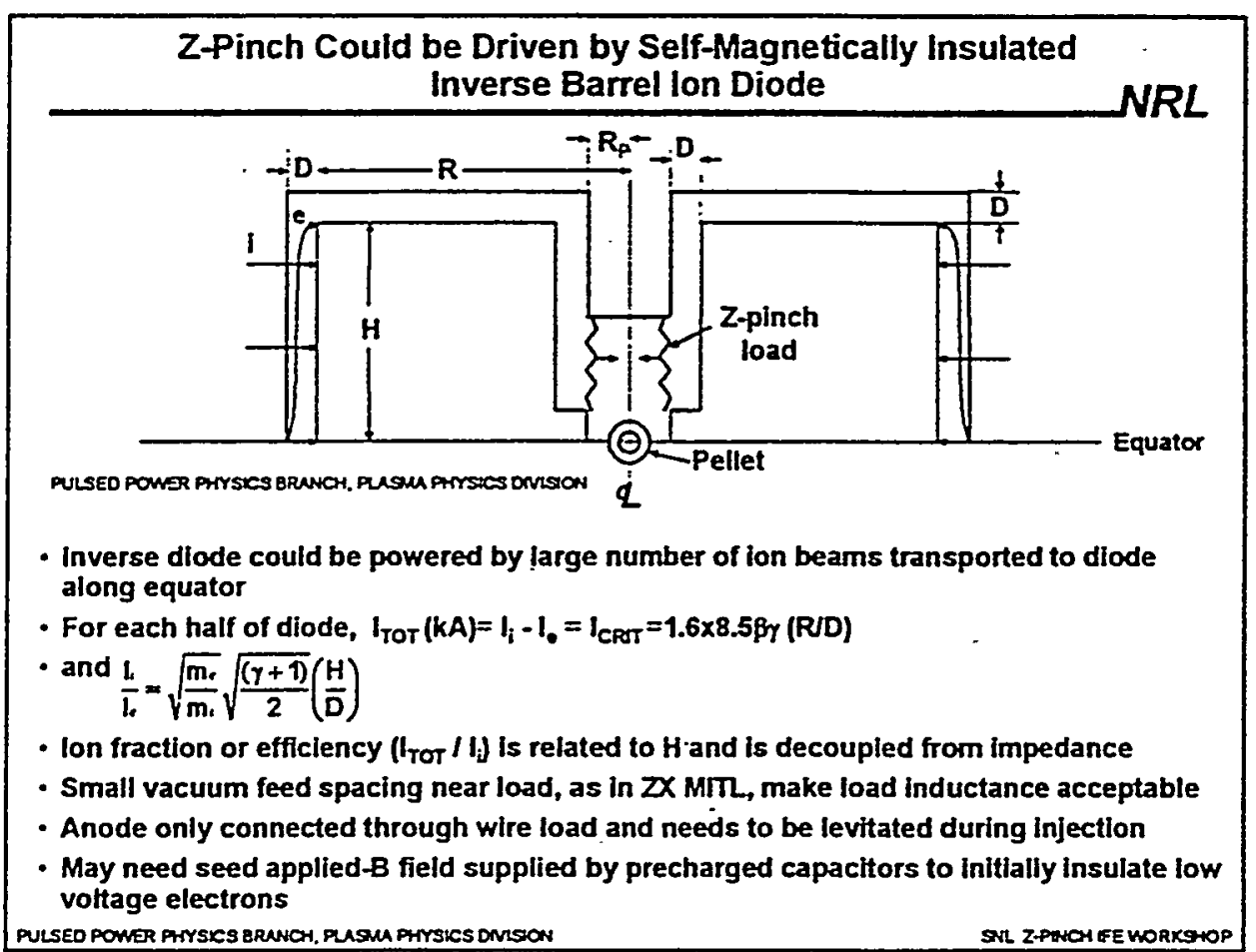

Page 4 


\section{Point Design}

$N R L$

lon Source

Assume $10 \mathrm{MV}, 100 \mathrm{MA}, 50$ ns unbunched proton beam produced by ten self-magnetically insulated $10 \mathrm{MV}, 10 \mathrm{MA}$, $50 \mathrm{~ns}$ ion diodes.

For $I_{\text {Tor }}(k A)=I_{1}+I_{e}=I_{C R r}=1.6 \times 8.5 \beta y(R / D)$ and $y=1+2^{\star} M V=21$

$\Rightarrow R / D=35$

Thus, for $D=0.5 \mathrm{~cm}, R=17.5 \mathrm{~cm}$

Ion fraction given by $\frac{l}{l}=\sqrt{\frac{m_{l}}{m_{1}}} \sqrt{\frac{(y+1)}{2}}\left(\frac{R}{D}\right)$

Assume additional factor of 2 for reflexing.

$\Rightarrow I_{1} I_{T}=l_{1} /\left(l_{1}+l_{Q}\right)=0.84$

Inverse diode

-Assume self-magnetically insulated barrel diode with radius $=R$, height $=H$, and $A K$ gap $=D$

-Each half of diode must operate at $10 \mathrm{MV}$ and $50 \mathrm{MA}$

-Assume $I_{\text {ror }}(K A)=I_{i}-I_{e}=I_{C R T}=1.6 \times 8.5 \beta \gamma(R / D)$

$\Rightarrow R / D=175$

-Thus, for $D=0.2 \mathrm{~cm}$ (as in MITL feeds), $R=35 \mathrm{~cm}$

-Ion fraction given by

$$
\frac{l}{l}=\sqrt{\frac{m}{m_{1}}} \sqrt{\frac{(\gamma+1)}{2}}\left(\frac{H}{D}\right)
$$

-For efficient capture, ássume $l_{T} h_{1}^{2}=\left(l_{1}-1_{e}\right) n_{1}=0.84$

$\Rightarrow I, \Omega_{e}=6.25$ and $H / D=81$

-Thus, for $D=0.2 \mathrm{~cm}, H=16 \mathrm{~cm}$

-Target and source size comparable and target easy to hit

-Dominant inductance is $L \approx 2 H$ in $\left(\left(R_{p}+D\right) / R_{p}\right)$ where $R_{p}=$ initial array radius

-Thus $L \approx 6 \mathrm{nH}$ for $R_{p}=1 \mathrm{~cm}, D=0.2 \mathrm{~cm}$

$\Rightarrow \mathrm{L} \mathrm{dl} / \mathrm{dT}=6 \mathrm{MV}$ 


\begin{tabular}{l} 
Point Design (cont.) \\
\hline Source divergence and transport \\
-For ballistic transport \\
-Assume source focuses onto target $5 \mathrm{~m}$ away \\
-Since target height is $\pm 16 \mathrm{~cm}$, allowed source divergence is \\
$\theta= \pm 32$ mrad \\
-Ballistic focusing followed by self-pinch transport would allow \\
even larger source divergence \\
-For self-pinch or guided transport \\
$I_{c h}=I_{A} \theta^{2}$ and \\
$I_{A}=31.3 \beta_{1} r_{1}(A / Z)=4.57$ MA for $10 \mathrm{MV}$ protons \\
-Thus, $I_{c h}<200 \mathrm{kA}$ for $\theta<200 \mathrm{mrad}$ \\
.
\end{tabular}

\begin{tabular}{l} 
Ion Source Options \\
\hline - Self-magnetically insulated ion diodes \\
- self-pinched electron beam diode enhances electron lifetime by RID \\
- pinch-reflex diode enhances electron lifetime via magnetic reflexing \\
- "backless" or low-mass-anode diode enhances electron lifetime via \\
electrostatic reflexing from virtual cathode \\
- for all of above, magnetic bending or defocusing due to AK gap closure \\
is partially compensated for during pulse by decreased bending distance \\
- Applied-B extractor ion diode \\
- MAP Applied-B extractor ion diode with ionized gas anode \\
- Higher-Z ions from two-stage applied-B extractor ion diode with \\
stripping to enhance current in inverse diode - suggested by Slutz \\
- All of above need preformed active anode plasma through plasma \\
injection or gas ionization \\
- All of above could possibly benefit by active plasma fill with AK gap \\
naturally forming in plasma sheaf
\end{tabular} 


\section{Ion Transport Options}

- Ballistic transport

- plasma created by beam-induced gas breakdown provides charge and current neutralization

- beam can be ballistically transported and focused with solenoidal lens

- Z-discharge transport in preformed plasma channel

- beam charge and current neutralized with confinement provided by discharge current

- laser initiated channels as considered in HIF schemes

- low-mass wall-confined channels

- Self-pinched transport

- requires plasma created by beam-induced gas breakdown to provide total charge and partial current neutralization

- net beam current provides beam confinement

- Wire guided transport

- beam charge and current neutralized with confinement provided by wire current

- Ballistic transport and focusing can be followed by guided transport

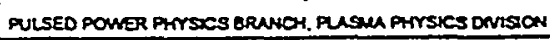
SNL Z-PACH gFE WORKSHOP

\section{Inverse Ion Diode Options}

$N R L$

- Self-magnetically insulated barrel diode

- because of complex electron orbits, it might be possible to exceed critical current leading to smaller diodes

" need PIC simulation

- may require seed applied-B field to initially insulate low voltage electrons $x$ could be supplied by small precharged capacitor attached to diode

- Applied-B magnetically insulated bartel ion diode

- magnetic field could be supplied by larger precharged capacitor

- virtual anode can be neutralized by electrons leaking across magnetic field lines as suggested by Slutz et al

- ion current density limitations might make diode dimensions unreasonable

- Both diode options could be powered by large number of ion beams transported to diode along equator

- Coaxial versions of both diodes could easily be tested on present facilities 


\section{Issues}

NRL

- To numerous to elaborate

- Major issue is survivable rep-rated ion diode

- need proof-of-principle experiments coupled with numerical simulation for scaling 


\title{
SYSTEM ISSUES FOR GENERATING AND DELIVERING PULSES IN Z-PINCH FUSION REACTORS
}

\author{
P.J. Turchi \\ The Ohio State University \\ Columbus, Ohio, USA
}

Z-Pinch IFE Workshop, 27 - 28 April 1999

Sandia National Laboratories, Albuquerque, NM 


\section{SYSTEMISSUES FOR Z-PINCH REACTORS}

WE MUST CONSIDER SYSTEM ISSUES FOR FUSION POWER REACTORS BEFORE HEADING DOWN TECHNICAL PATHS THAT WILL NOT LEAD TO ACCEPTABLE CONCEPTS.

PRINCIPAL COMPLAINTS OF POWER COMPANIES REGARDING FUSION

--- Power level for the initial installation is too high.

For acceptable circulating power-fraction $(\leq 15 \%)$, conventional fusion concepts must operate at power levels of several gigawatts ( large city).

--- Complexity of systems increases costs relative to competing techniques (e.g., coal, natural gas), both capital and operating.

Plurality of coils, beam- and EM- injectors; vacuum systems; chemical processing for tritium extraction.

-- Induced radioactivity of first-wall leads to waste problems again. 


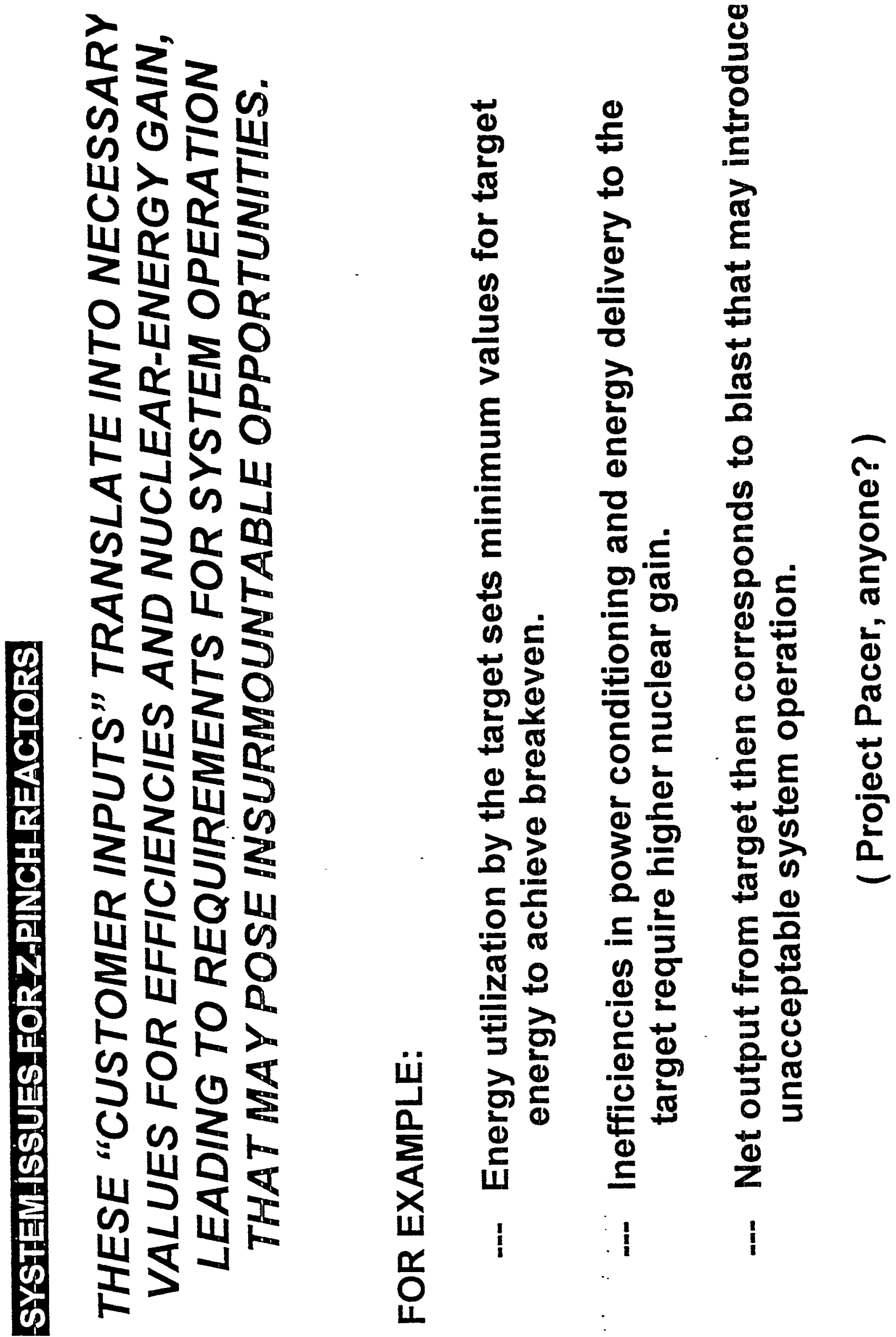



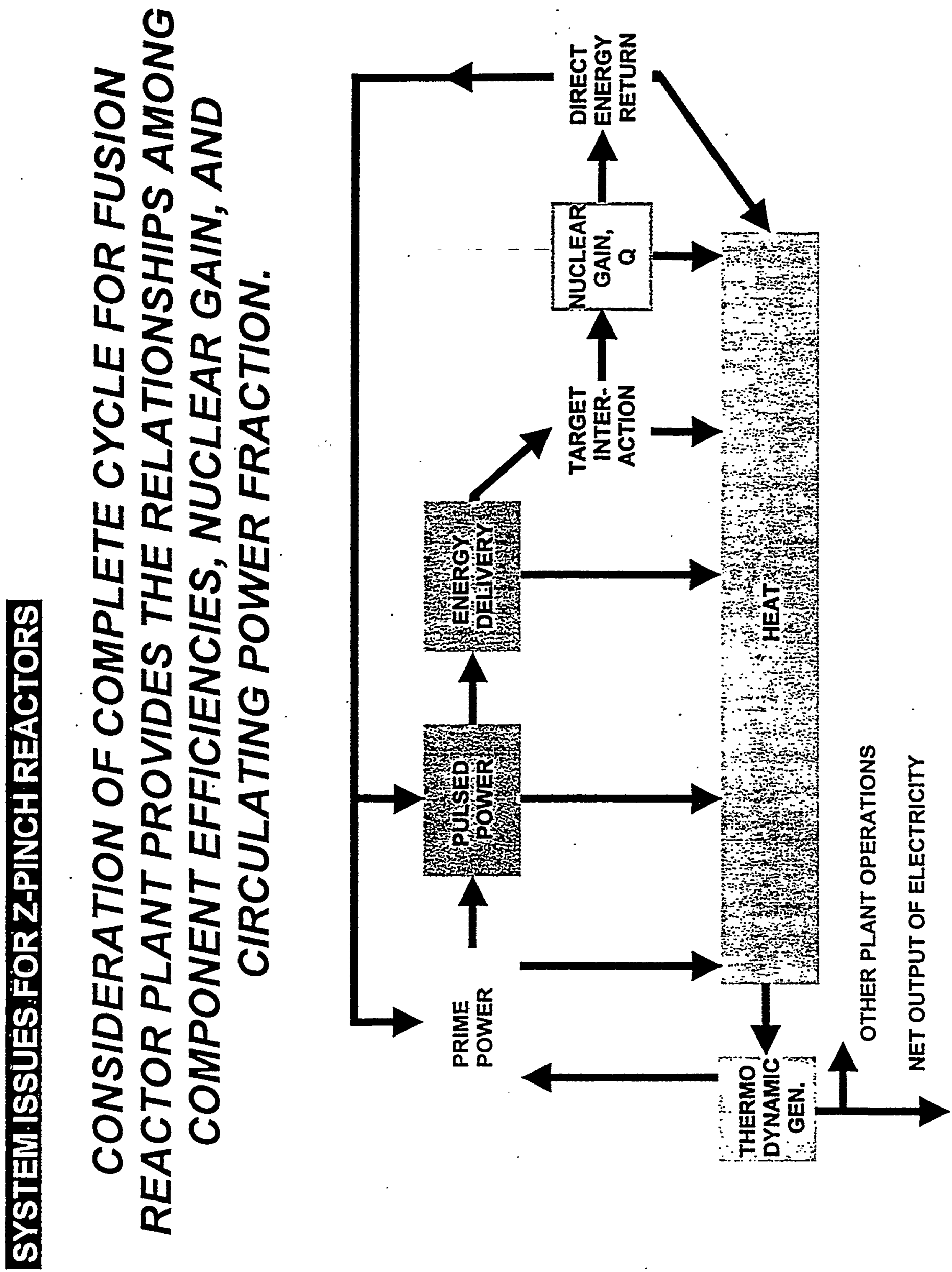


\section{SYSTEM ISSUES FOR Z-PINCH REACTORS}

CALCULATION OF THE NECESSARY NUCLEAR GAIN, $Q$, SHOULD INCLUDE ALL THE WAYS OF USING ENERGY.

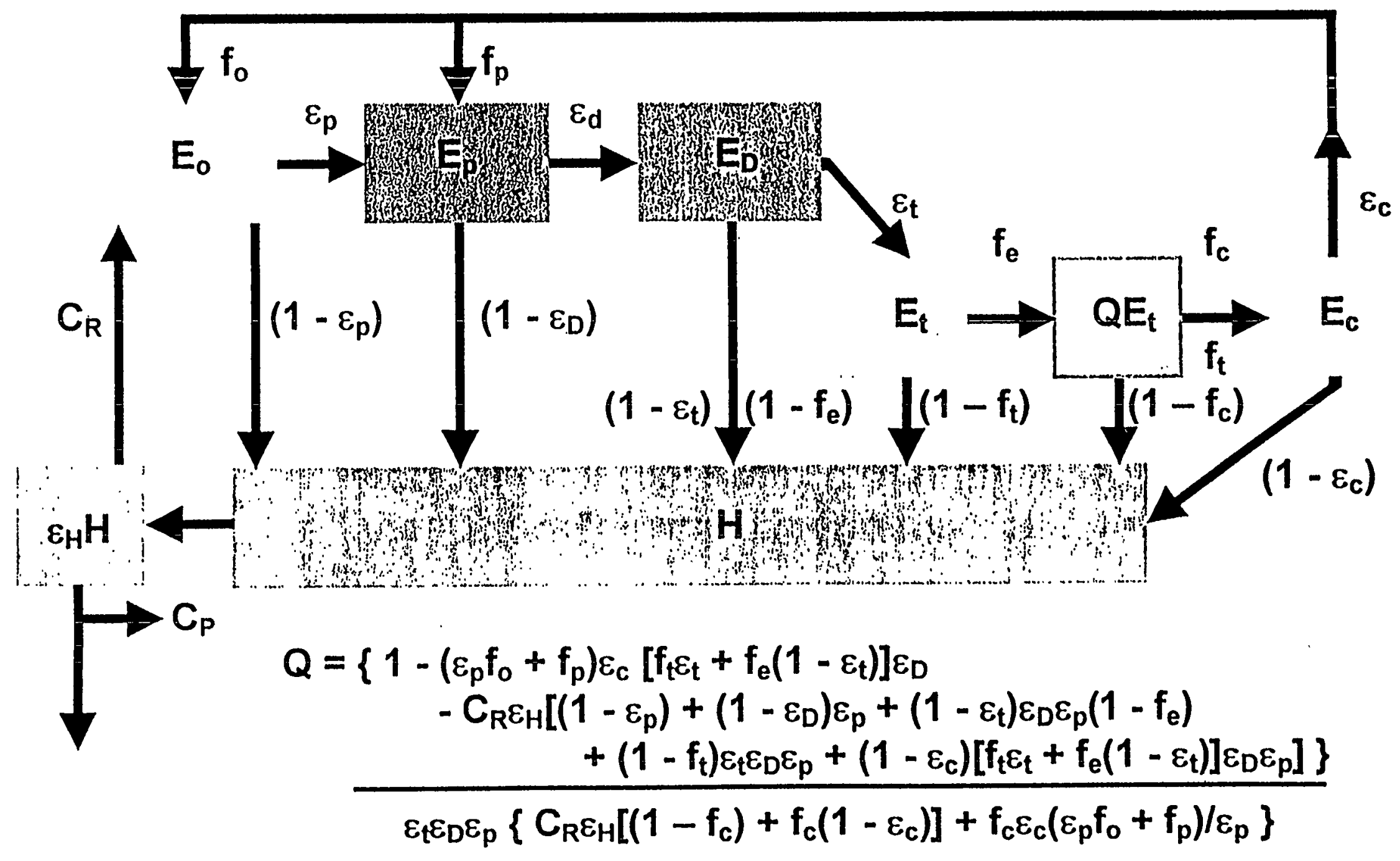




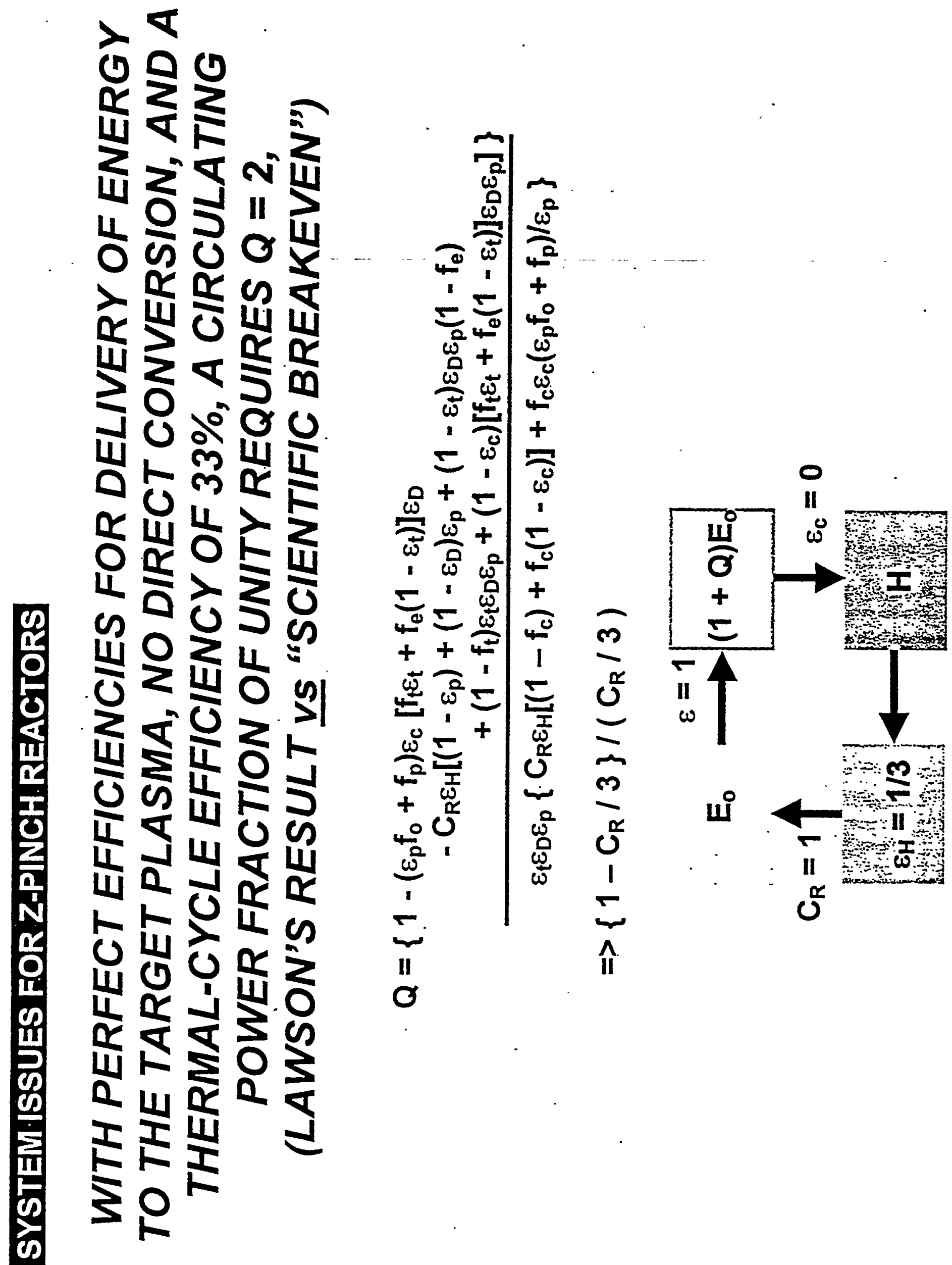




\section{SYSTEMMISSUES-EORZZ:PINCHAREACTORS}

RECOVERY OF PORTION OF TARGET ENERGY (INCLUDING NUCLEAR ENERGY) DIRECTLY TO THE DRIVER SYSTEM CAN SUBSTANTIALLY REDUCE THE NECESSARY GAIN NEEDED TO OBTAIN A DESIRED CIRCULATING POWER FRACTION.

Sample values for efficiencies and other factors:

Prime power to pulsed power system, $\varepsilon_{p}=1.0$, Pulsed power delivered, $\varepsilon_{D}=0.75$ Energy absorbed by target, $\varepsilon_{t}=1.0$, Efficiency of thermodynamic cycle, $\varepsilon_{H}=0.33$ Fraction of nuclear energy deposited in plasma, $f_{c}=0.15$

Fraction of target energy available for direct conversion, $f_{t}=1.0$ Fraction of unabsorbed energy available for direct conversion, $f_{e}=0.5$ Fraction of direct conversion returned to prime-power, $f_{0}=0.5$ Fraction of direct conversion returned to pulsed-power, $f_{p}=0.5$ Circulating Power Fraction for Reactor, $C_{R}=0.1$

Without Direct Conversion $\left(\varepsilon_{c}=0\right): Q=38.7$

With Direct Conversion $\left(\varepsilon_{c}=0.8\right): Q=3.45$ 


\section{SYSTEMIISSUES.FOR Z-PINCH REACTORS}

RECOVERY OF PORTION OF TARGET ENERGY (INCLUDING NUCLEAR ENERGY) DIRECTLY TO THE DRIVER SYSTEM CAN COMPENSATE FOR MORE REALISTIC VALUES OF EFFICIENCIES OF ENERGY DELIVERY.

Sample values for efficiencies and other factors:

Prime power to pulsed power system, $\varepsilon_{p}=0.9$, Pulsed power delivered, $\varepsilon_{D}=0.5$ Energy absorbed by target, $\varepsilon_{t}=0.3$, Efficiency of thermodynamic cycle, $\varepsilon_{H}=0.33$ Fraction of nuclear energy deposited in plasma, $f_{c}=0.15$ Fraction of target energy available for direct conversion, $f_{t}=1.0$ Fraction of unabsorbed energy available for direct conversion, $f_{e}=0.5$ Fraction of direct conversion returned to prime-power, $f_{0}=0.5$. Fraction of direct conversion returned to pulsed-power, $f_{p}=0.5$ Circulating Power Fraction for Reactor, $C_{R}=0.1$

Without Direct Conversion $\left(\varepsilon_{c}=0\right): Q=217$

With Direct Conversion $\left(\varepsilon_{c}=0.8\right): Q=34.6$ 


\section{SYSTEMISSUES-FORZZPINGH-REACTORS}

EMPHASIS ON EFFICIENCY OF ENERGY DELIVERY MAY PRECLUDE MORE INTERESTING SCHEMES FOR POWERING

A Z-PINCH LOAD, SUCH AS REPEATED EXCHANGES OF ELECTROMAGNETIC ENERGY AND KINETIC ENERGY.

--- Use of Z-pinch already depends on efficient transfer of electromagnetic energy to kinetic energy and then to radiation.

--- Magnetic-flux compression techniques can concentrate energy and power, but at the expense of resistive losses.

MCGs count on initial source of high energy in form of "cheap" explosives. Economics of a power reactor not important.

Virtues of magnetic-flux compression include relatively low voltages, and ability to transfer energy without high current connections.

--- Inverse-diode needs proper "target" region during bombardment by high energy beam, but could revive beam technology. 


\section{SYSTEM ISSUES FOR Z-PINCH REACTORS}

\section{MAGNETIC FLUX COMPRESSION TECHNIQUES MIGHT INCORPORATE LIQUID-METAL LINERS TO ACT AS FUSION REACTOR BLANKET AND ALSO MECHANISM FOR DIRECT CONVERSION OF PORTION OF NUCLEAR ENERGY.}

LINER-DRIVEN GENERATOR

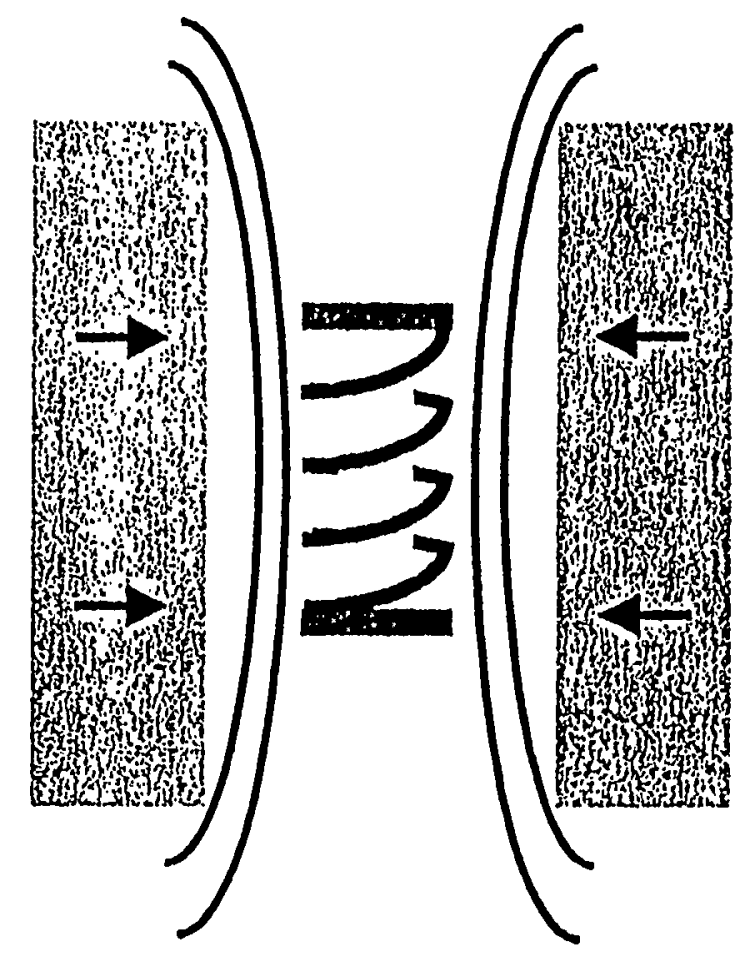

\section{LINER WITH PLASMA FLOW SWITCH}

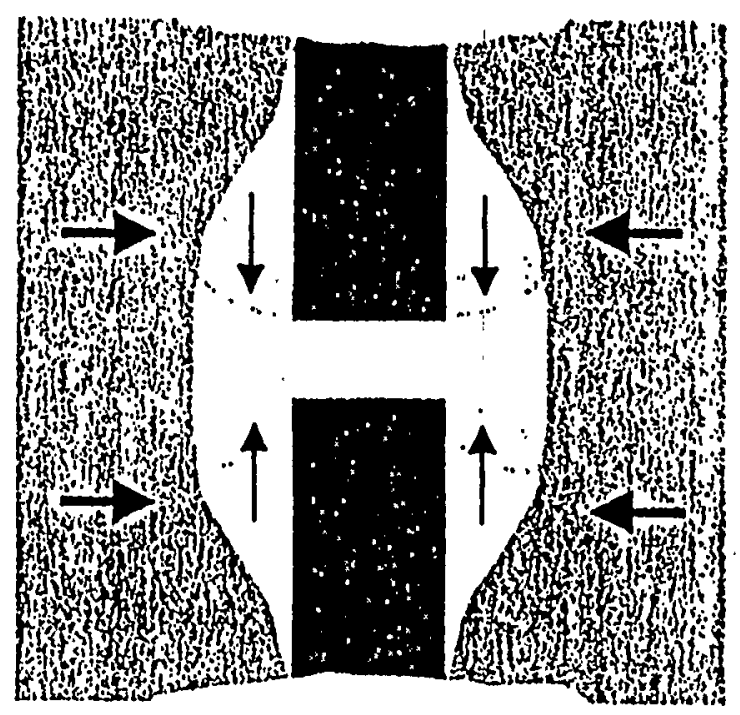




\section{SYSTEMISSUES FOR ZEPINCH REACTORS}

\section{SUMMARY REMARKS}

--- Prospects for fusion power reactors based on Z-pinch technology depend critically on the efficiencies of delivering energy to the target plasma. Reliability over 100 million shots is also key.

--. Direct conversion of a portion of nuclear energy and recovery of plasma and other energy without requiring circulation of power through the thermodynamic generator substantially reduces the necessary nuclear gain. This greatly improves the reactor acceptability.

-- Use of a stabilized liquid-metal implosion (as in LINUS reactor design) as a pulsed power element may provide an approach that permits repetitive operation with large amounts of energy per pulse. It also offers attractive reactor features, such as energy recovery, blast mitigation and tritium breeding without a first-wall. 


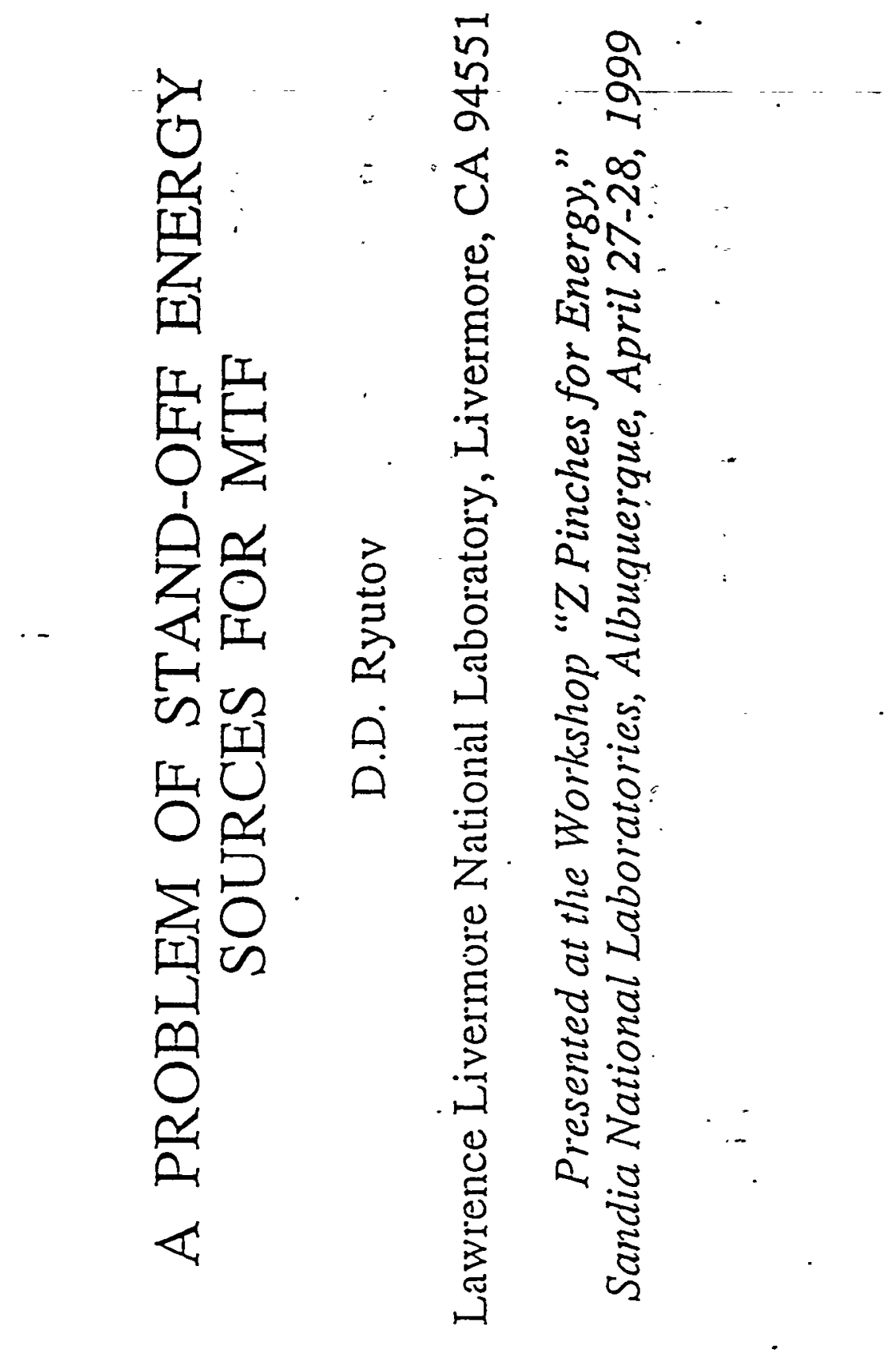


MTF concept encompasses a broad range of fusion systems, with the yields from many gigajoules per pulse to a few megajoules per pulse, and with rep-rates from $\sim 0.1 \mathrm{~Hz}$ to $>10 \mathrm{~Hz}$.

We discuss here energy sources for the fusion systems of the type of cmsize imploding liners, with a total energy input 1-10 MJ and a pulsewidth $\sim$ a few microseconds

The current should be in the range of $5-10 \mathrm{MA}$.

The expected fusion gain is $\sim 10-30$, meaning that the energy release per pulse is $\sim 100 \mathrm{MJ}(30 \mathrm{kWh}$ only!) 


\section{A CONCEPT OF AN "ASSEMBLY"}

The "assembly" is an object containing a plasma creation system and a circuitry that allows conversion of the energy delivered to the assembly into the liner energy. Such "assemblies" would be dropped (or inserted into reaction chamber) at a desired rate and energized by a stand-off energy source. 


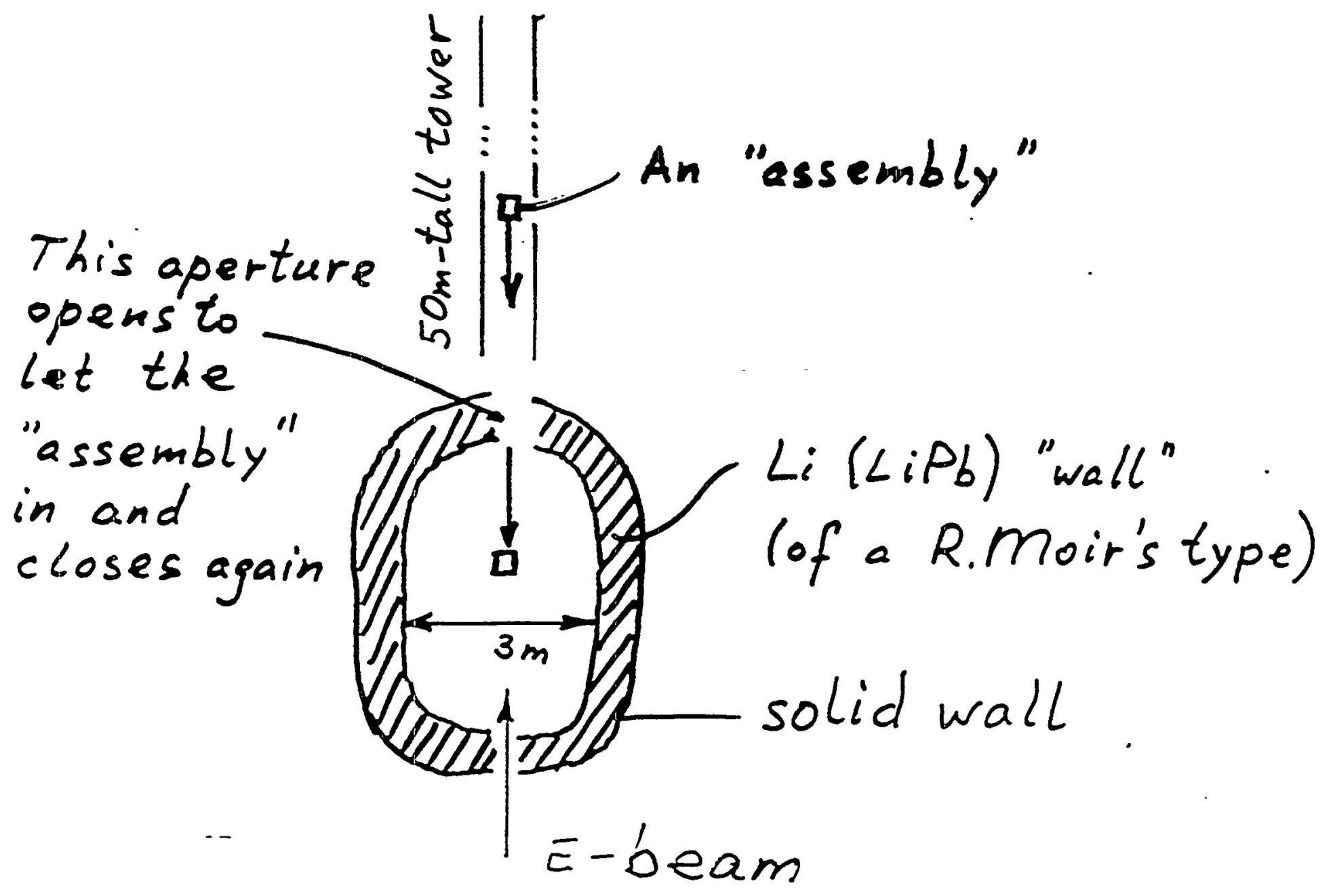

$a$ cost of each shot should be less than 0.5 cent per $1 \mathrm{MJ}$ of the energy released! 
How does the energy conversion work?

$E$-beam

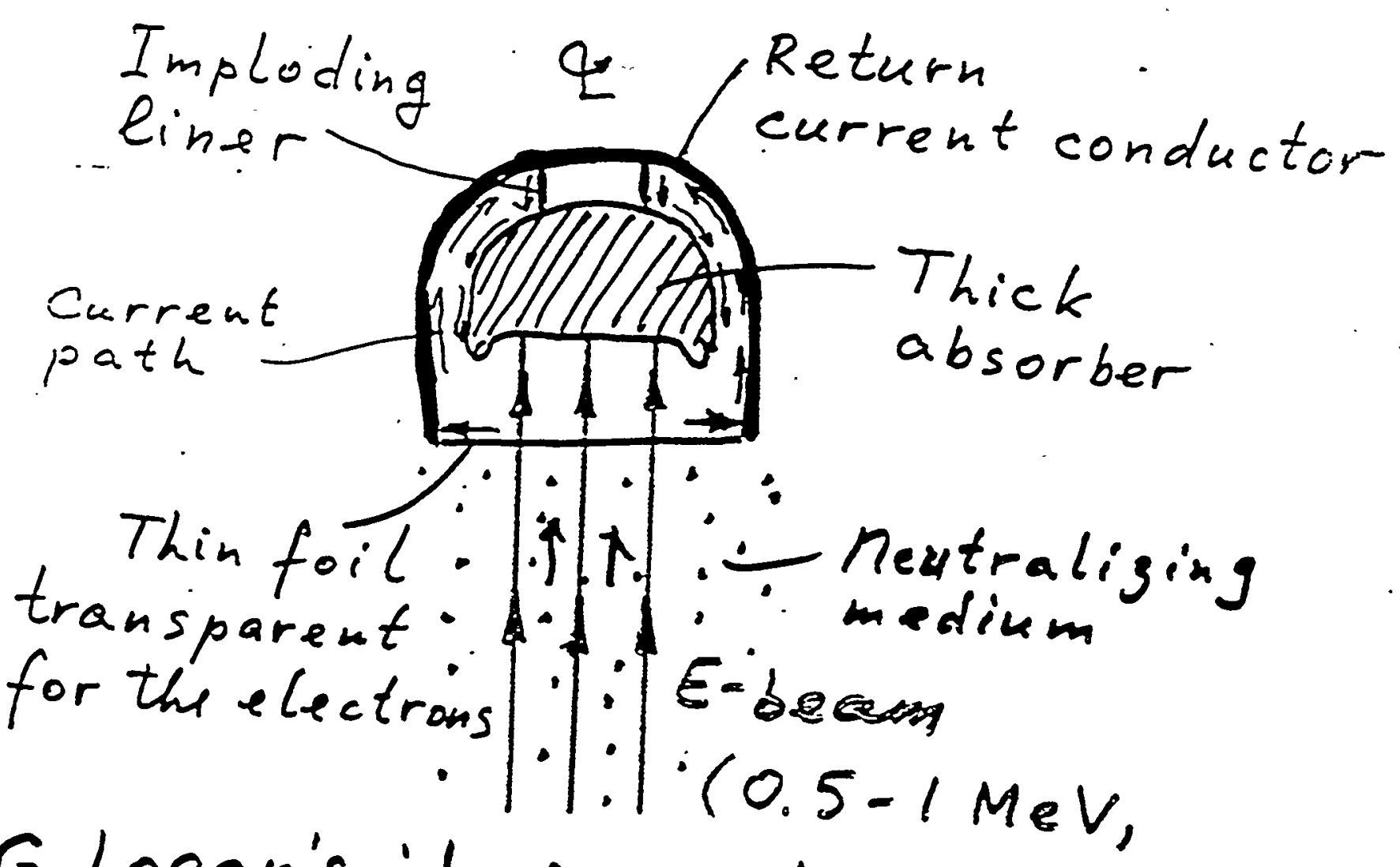

G. Logan's idea: $\quad M A, 3=3 \mu s)$

all the elements can be made of $L_{i}$ (except the liner).

The prototype beam sources do exist (Novosibirsk). 
- Distance to the target $\sim$ a few meters

- Compatibility with a liquid (say, LiPb) protective wall

- Sufficient rep-rate 


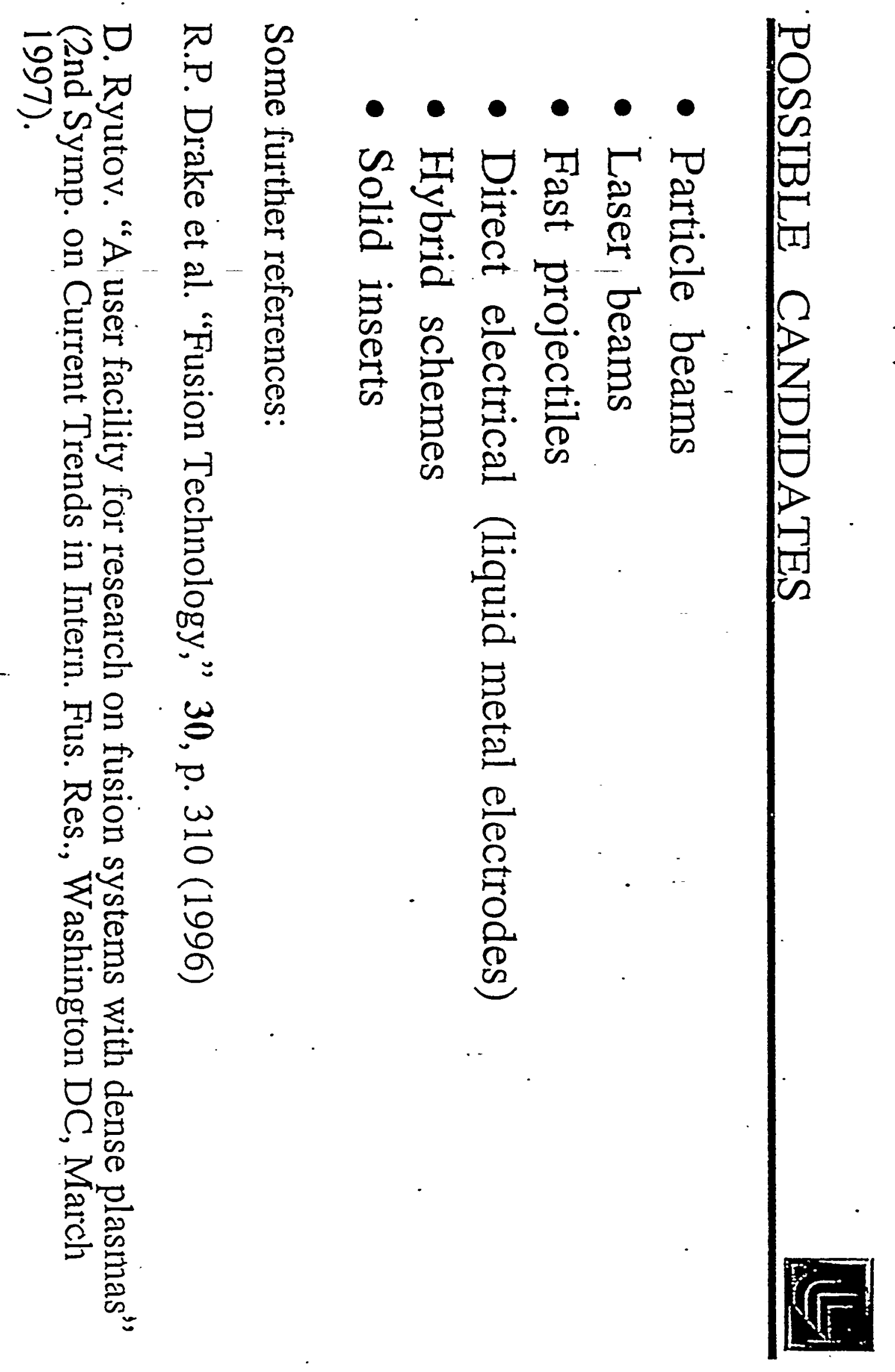


Lasers

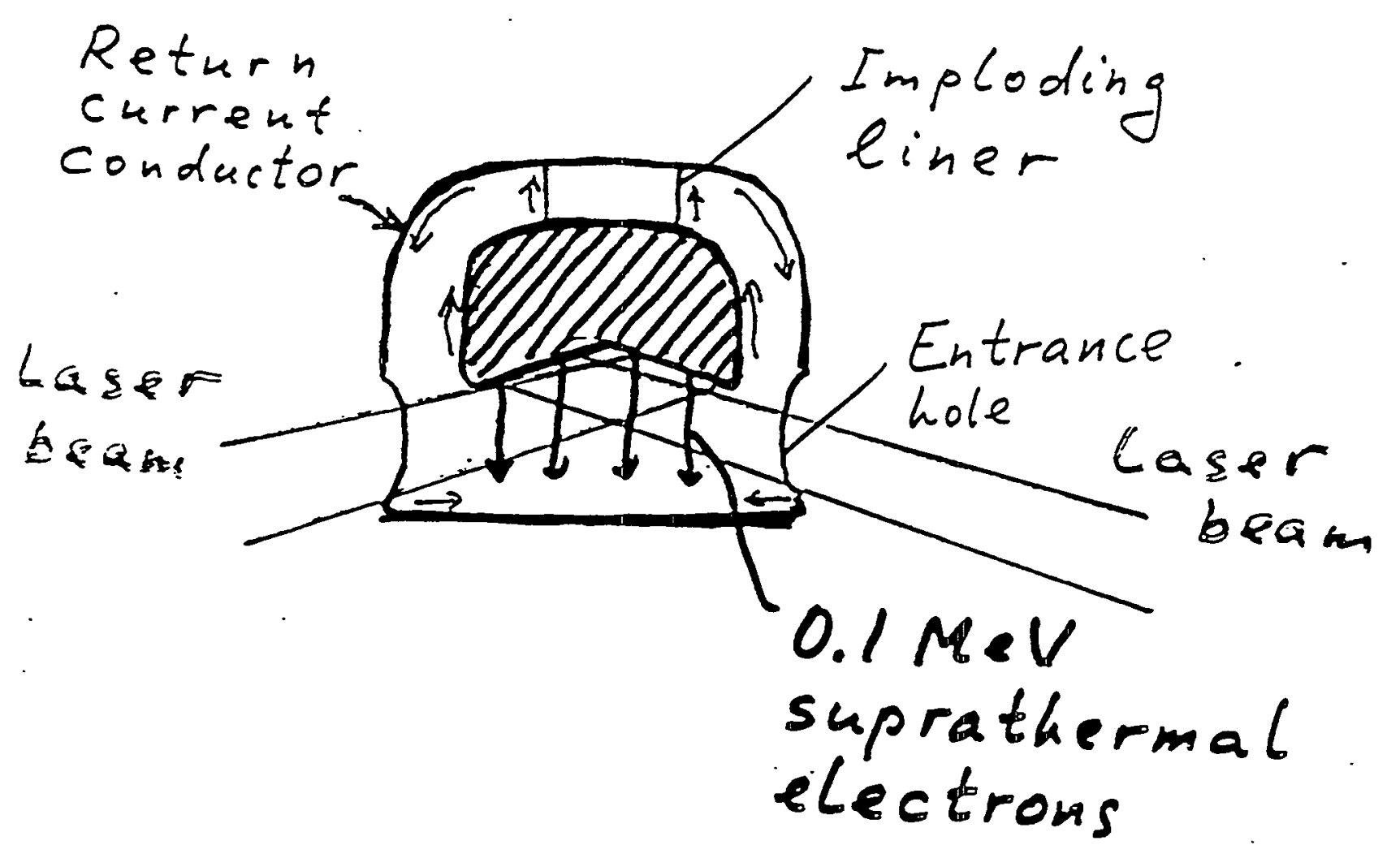

The actual shape can be different, with a Larger area allowed for the surface. illuminated by the lasers 
Fast projectiles

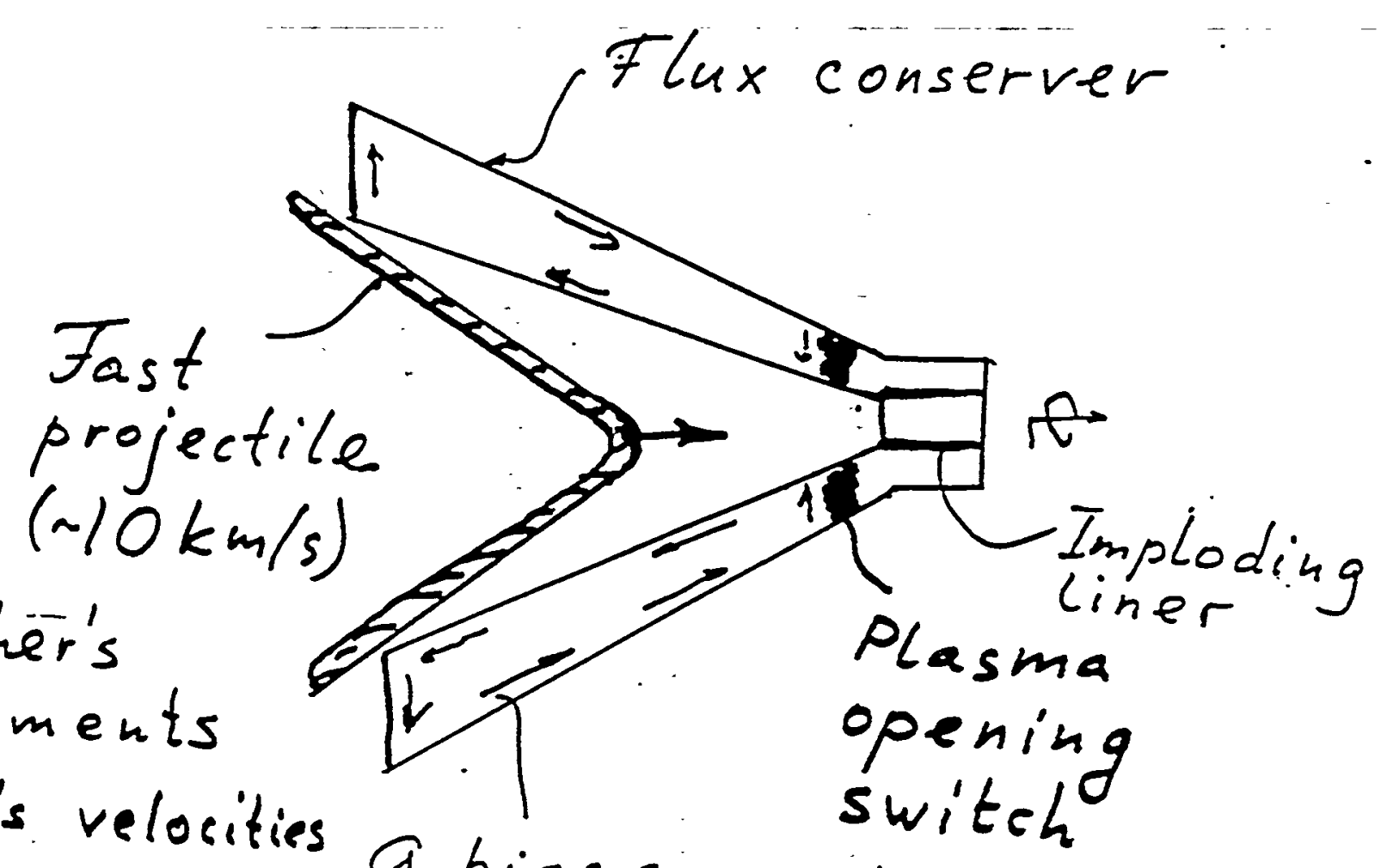

In J. Asher's

experiments

$10 \mathrm{~km} / \mathrm{s}$ velocities

have been reached $a$ bias current (1980's) (generated, egg., by the E-beam)

- Under the action of the

1007

$=4 \mathrm{~kJ} / \mathrm{cm}^{3}$ fast projectile, the inner wall of the flux conserved sticks to the outer wall, compressing magnetic flux Mechanical perturbations do not reach the liner before its implosion is over. 
Liquid Li electrodes

(A .Robson, 1980's)

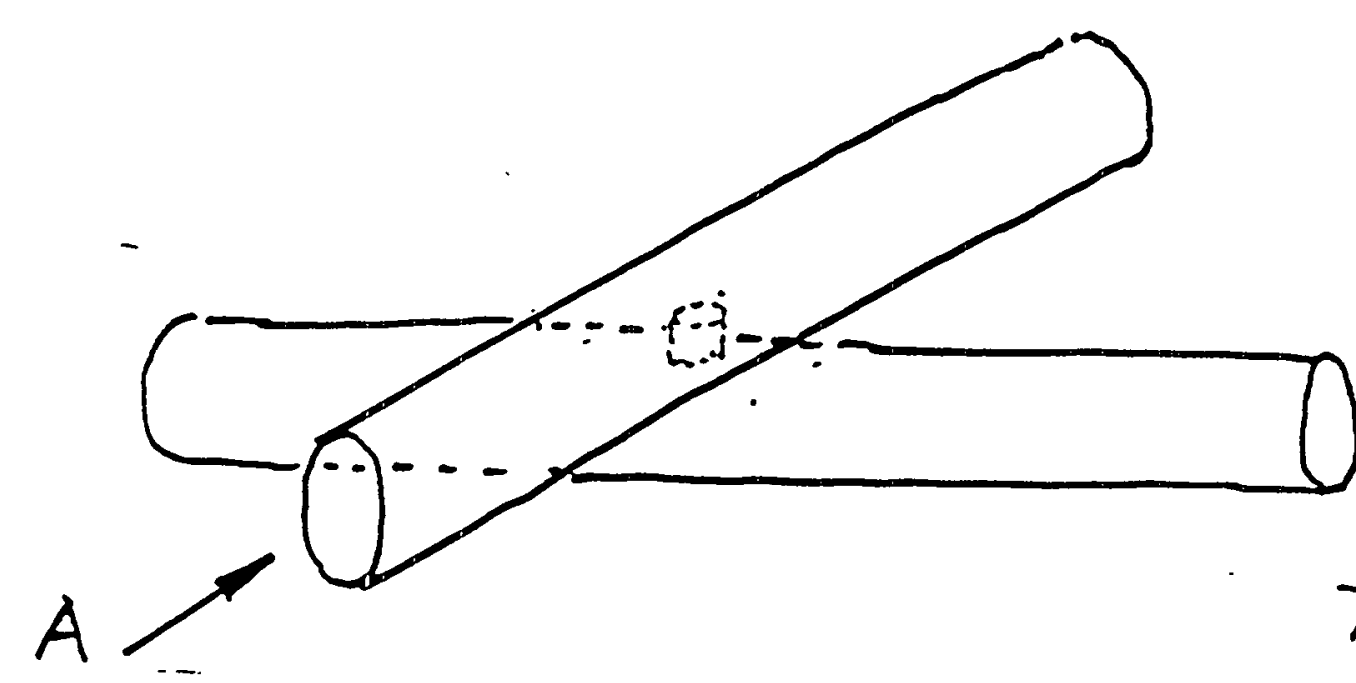

The lithium View along arrow $A$ : vapor is an insulator!

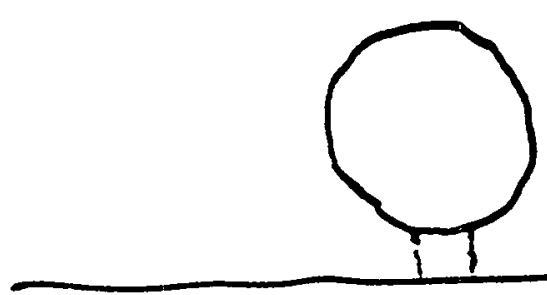

With the length of liquid electrode - a few meters, this system is too inductive to drive a current "I OMA. The use of an "assembly" with a builtin transformer is requires 
To avoid the need in chemical separation of the assembly material and the material of a protective layer, it is desirable to make the assembly of the same materials, say; Li (J. Hammer, B.G. Logan, private communication) 


\section{FURTHER DETAILS}

The assemblies may also have a more sophisticated on-board circuitry (transformers, switches)

Perhaps, $\mathrm{Li}$, or $\mathrm{LiPb}$, or $\mathrm{Pb}$ wires encapsulated into a properly chosen insulator can be developed

To intprove mechanical and electrical properties of the constrituction materials, one could cool the assemblies down to $-20 \mathrm{C}$ or lower temperatures 


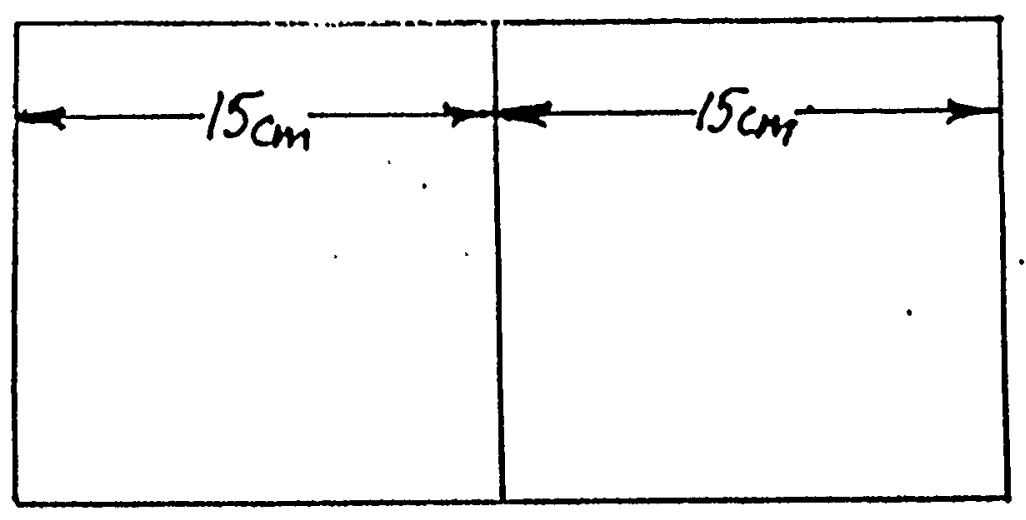

\section{Total mass $\sim 200 \mathrm{~g}$}

\section{FRC FORMATION SECTION}

- Bias magnetic field (a few $\mu \mathrm{s}, 10 \mathrm{~T}$, total energy $10-20 \mathrm{~kJ}$, required current $0.5 \mathrm{MA}$ )

- Field-reversal solenoid ( $1 \mu \mathrm{s}, 15 \mathrm{~T}$, total energy $10-20 \mathrm{~kJ}$, required current $0.7 \mathrm{MA}$ )

- Pre-ionization system (a hole for a laser pre-ionization?)

Possible power supplies: laser;

E-beam + transformer

\section{IMPLOSION SECTION}

- Intplosion chamber with a liner $\left(\mathrm{L}=5 \mathrm{~cm}, \mathrm{r}_{0}=1 \mathrm{~cm}\right.$, gold $+\mathrm{alu}-$ minum (?), $I_{\operatorname{mix}}=10 \mathrm{MA}$, current pulse-width 2-3 $\mu \mathrm{s}$ )

Possible power supply:

Flux-compression system driven by a fast projectile $(10 \mathrm{~g}, 20 \mathrm{~km} / \mathrm{s})$ $\mathrm{W}(\mathrm{MJ})=0.05 \cdot \mathrm{m}(\mathrm{g}) \cdot[\mathrm{v}(10 \mathrm{~km} / \mathrm{s})]^{2}$ 


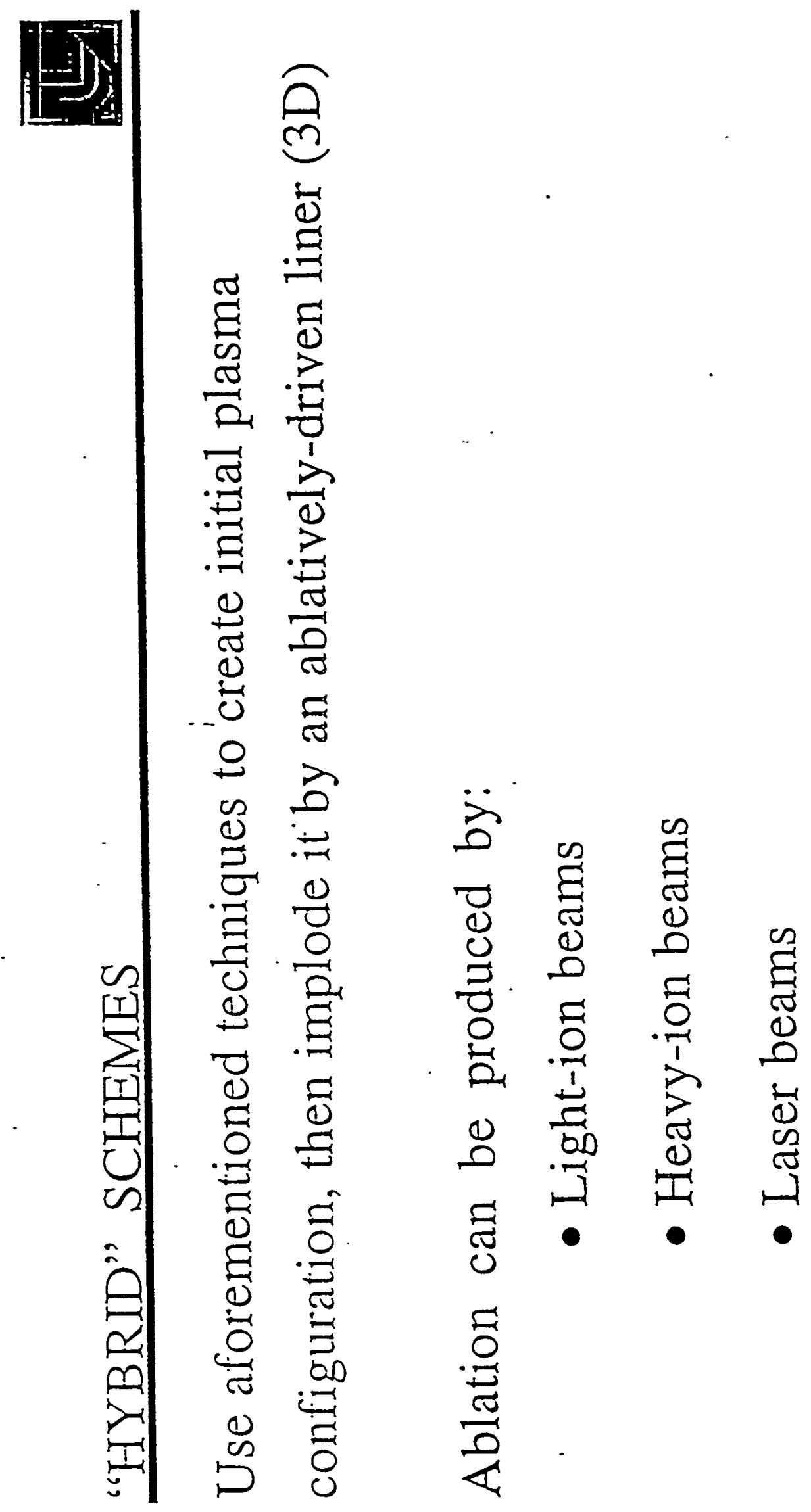




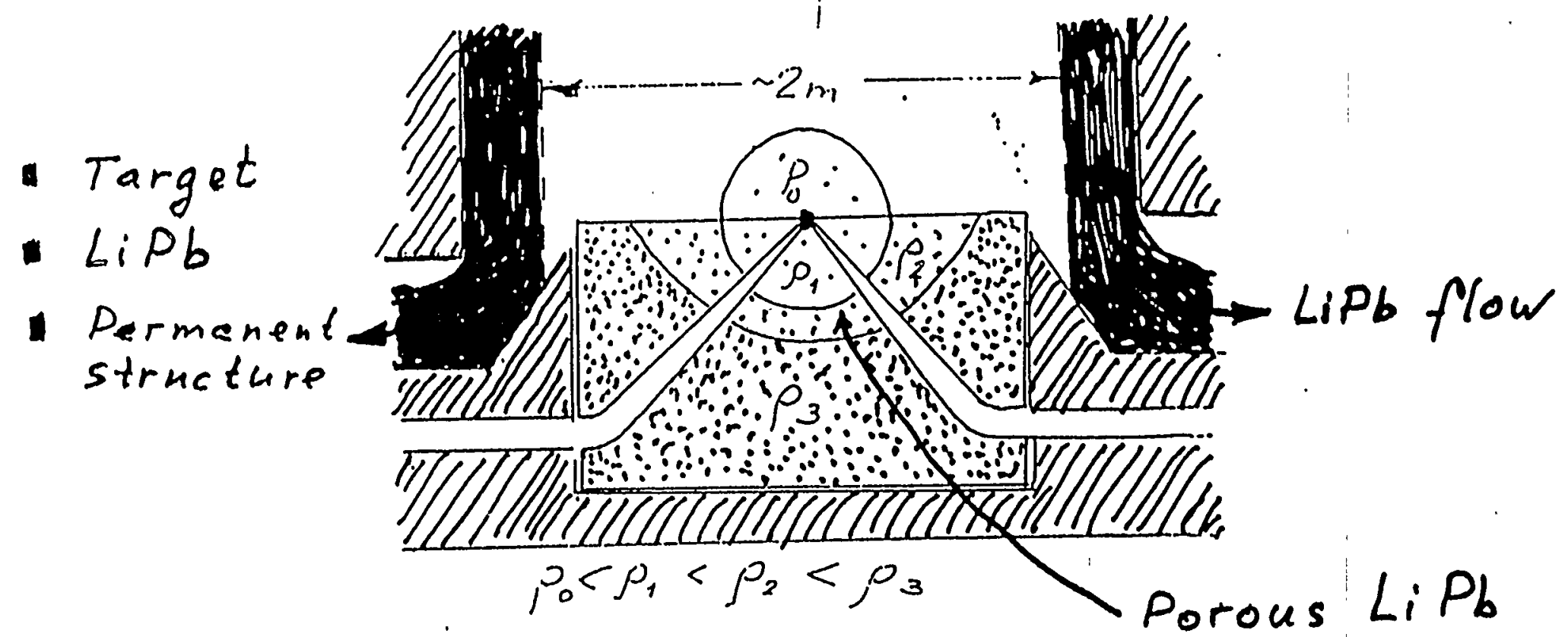

- The material: a porous $\mathrm{LiPb}$ (or $\mathrm{Li}$, or $\mathrm{Pb}$ ) at $\mathrm{T}<-20 \mathrm{C}$

- Density distribution in the vicinity the target chosen in such a way as to exploit the effect of hydrodynamical lensing and direct the ejected material away from the most vulnerable elements of the reaction chamber

- Replacement rate $\sim 1 \mathrm{~Hz}$ 


\section{SUMMARY}

- The problem of stand-off energy sources for MTF is in its infancy and it is unfair to ask for too much now

- On the other hand, there is (almost) no doubt that at least one (or more) of the aforementioned concepts will work in single-shot experiments

- A much more formidable task is to demonstrate a feasibility of mass production of disposable elements at low cost 


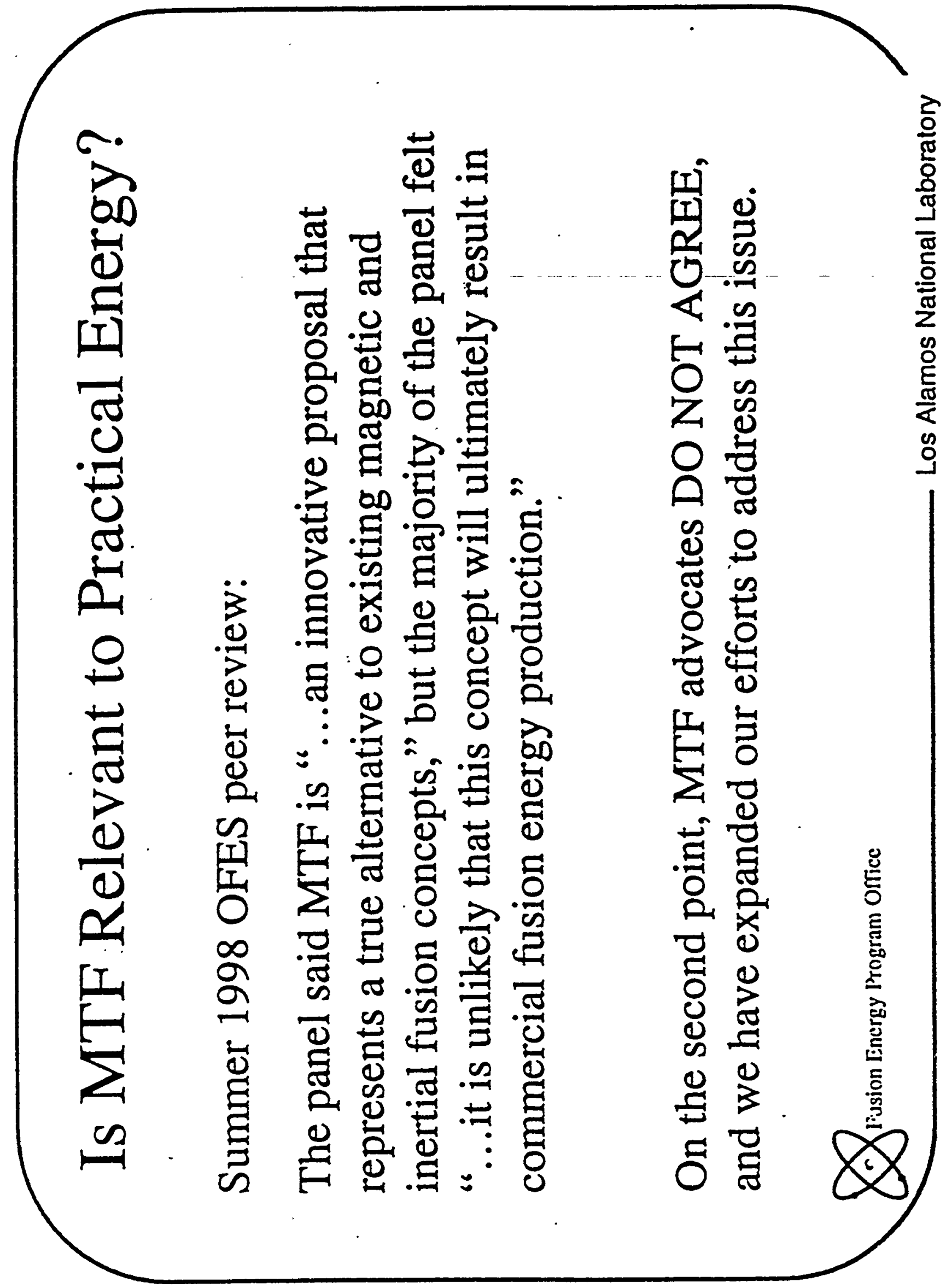




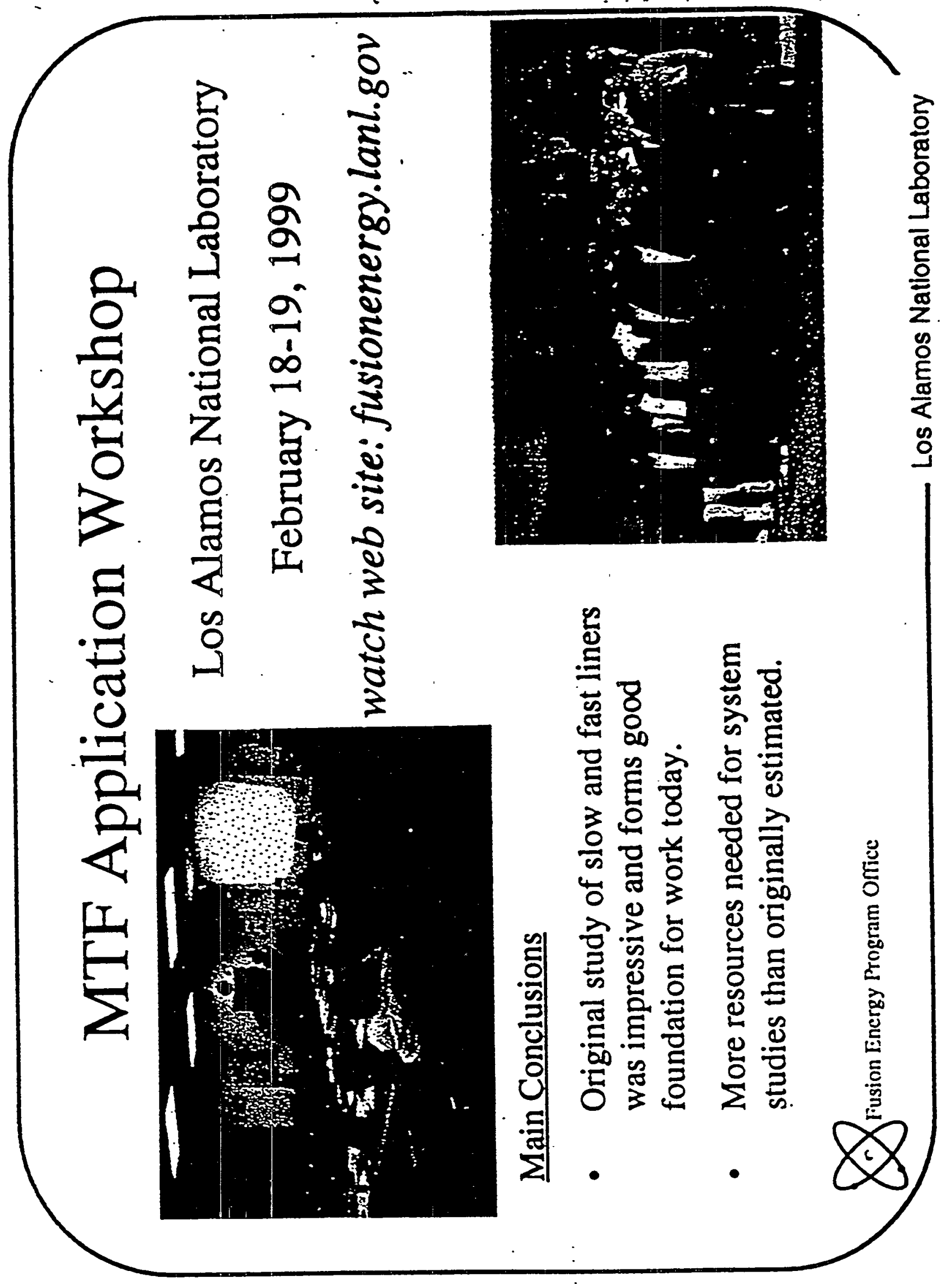




\section{LINUS: The survivable imploding liner}

Pioneers: R. Robson, P. Turchi at NRL see P. Turchi, "A Compact-Toroid Fusion Reactor Design at 0.5 Megagauss, Based on Stabilized Liner Implosion Techniques," in Ultrahigh Magnetic Fields: Physics, Techniques, Applications, V.M. Titov and G.A. Shvetsov, eds., Nauka, Moscow (1984).

Recent thinking: K. Fowler, (presented by D. Ryutov), proceedings of MTF Applications Workshop, Los Alamos, 1999. 


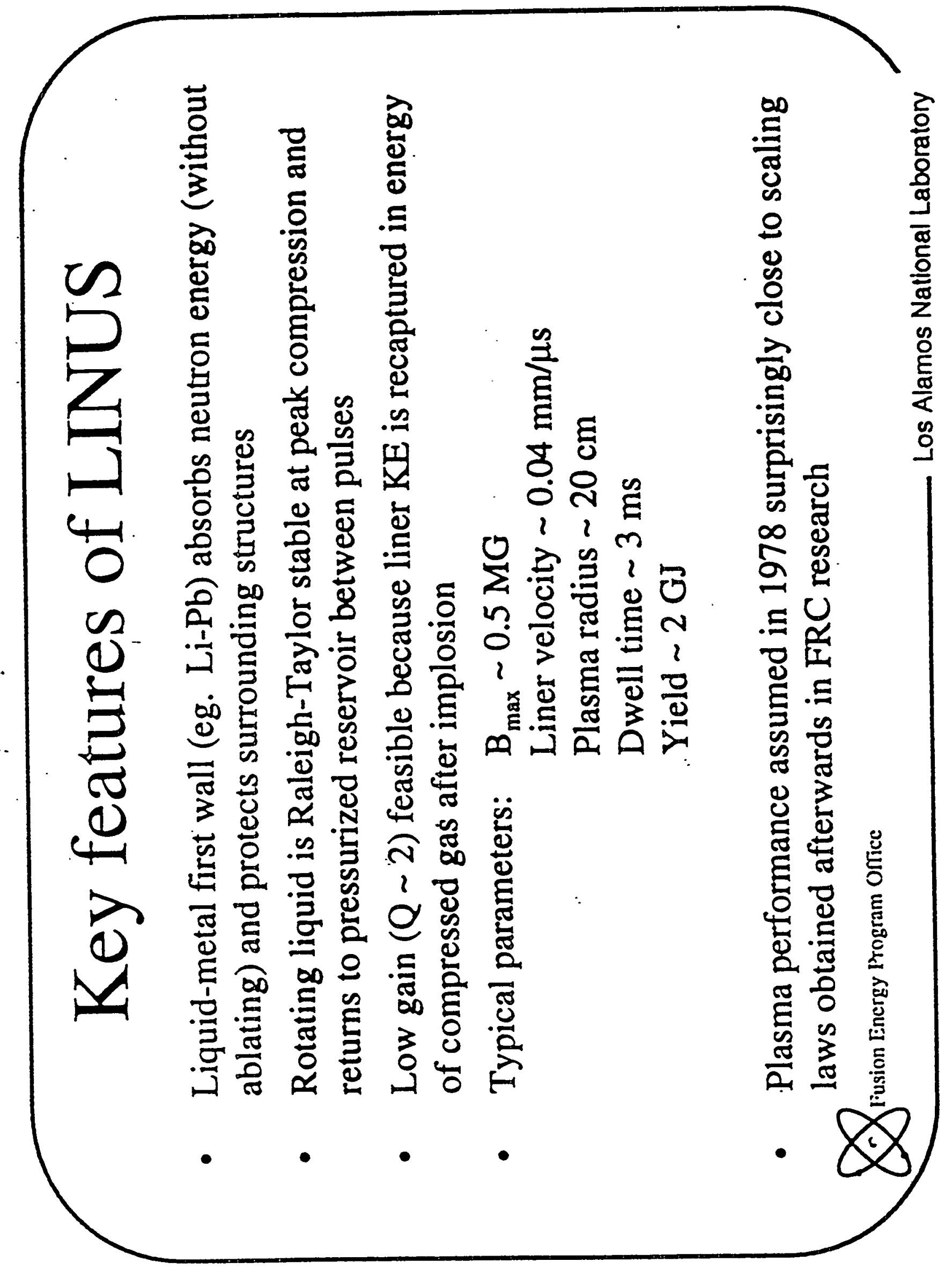




\section{Fast Liner Reactor}

A CONCLPTUNe OLSIGN OF THE FAST-LINEA

Rucion (FLR) FOR FUSIOH PONER

$$
\text { by } \text { tht }^{\text {th }}
$$

R. H. Moses, R. A. Krakowskl, and R. L. HIller

ASSTRNCT

The ganeration of pusion powar pron the falt-linar Resctor (PL) concept envisuges the inploslon of a $\operatorname{thln}$ (J. milallic cyllndor $(0.2-0$ redlus by $0.2-m$ length) onto proinfected plases. Mls plasene would be mated to therwonucles tomeratures oy adiabetle compresilon. pressure confinomit noold on provlated by the pliner

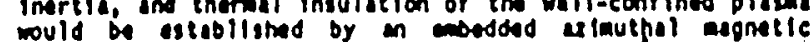

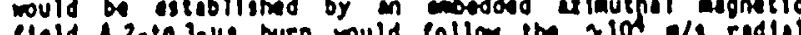

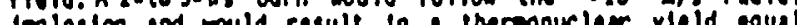

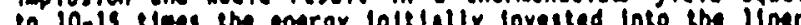
klnetle enerey for toplostone oceurring once avery 10 :

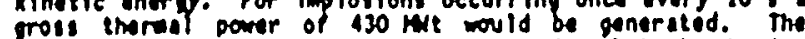
results of acoprenensive systems study of both physles and technoloay (econonies) opthe are presented. Dasples eechnology of the PLR. : conceptual power plent design is presented.

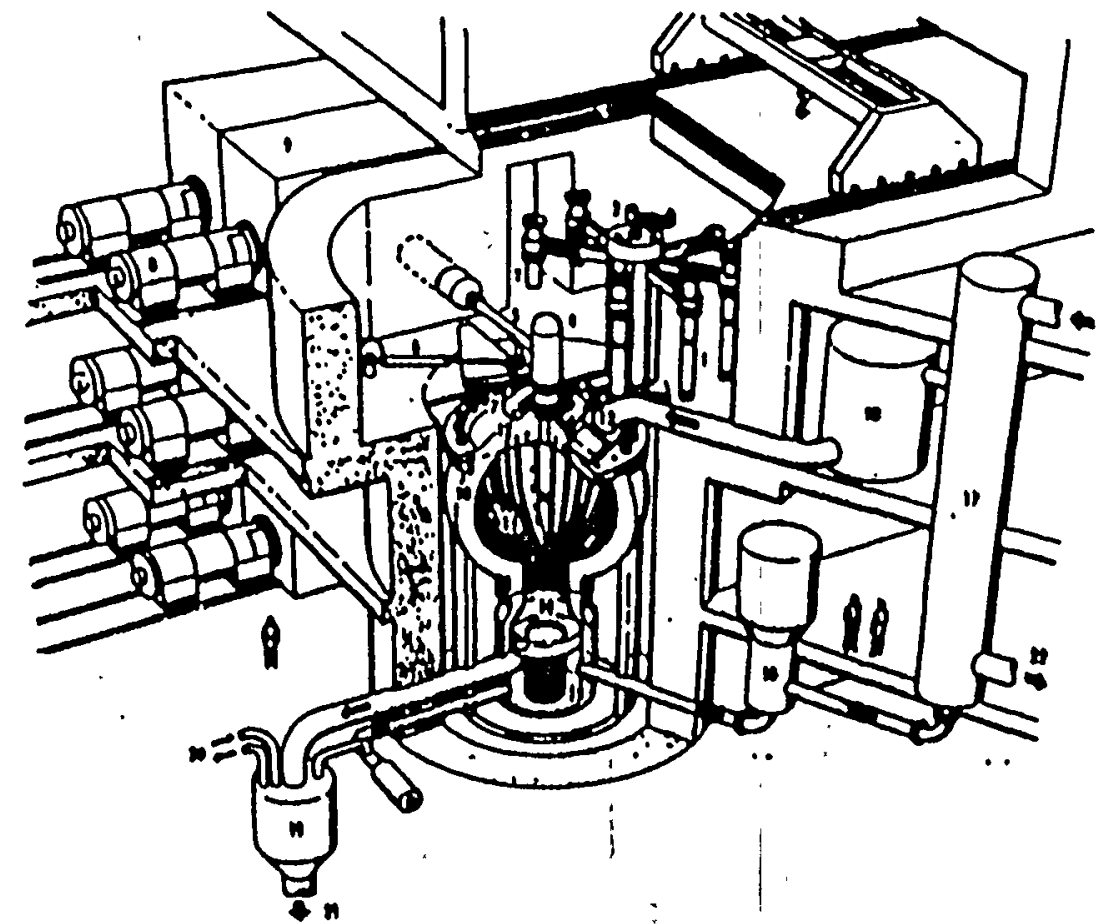

i

F19. 1l-1. Isouttrle ofutho of rast-LIndr Recetor nuclear Island for the

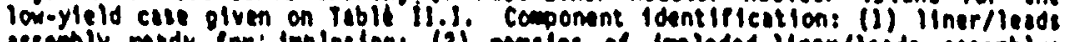

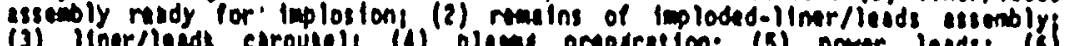
(3) Iinorlleeds caroulell (1) pleame pmparetion: (3) power leods: (6)

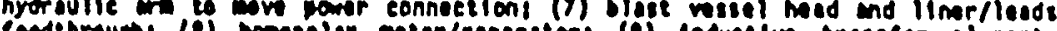

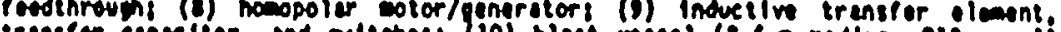

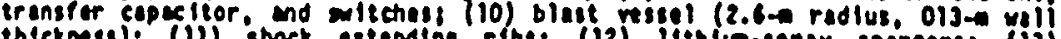
lientue iniet ond control velve: (14)

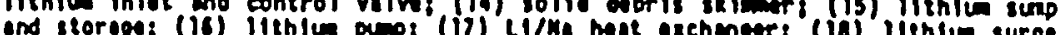

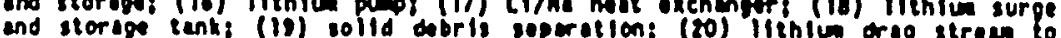
tritlue recovery: (21) sollds dobrls to rocovery and refabricationi (22) stecondary codiun coolent. 


\title{
Electrical power delivery needs no line-of-sight
}

\author{
Quartz \\ vacuum \\ envelope \\ filled with \\ DT gas
}

Precision liner Al or Be or?

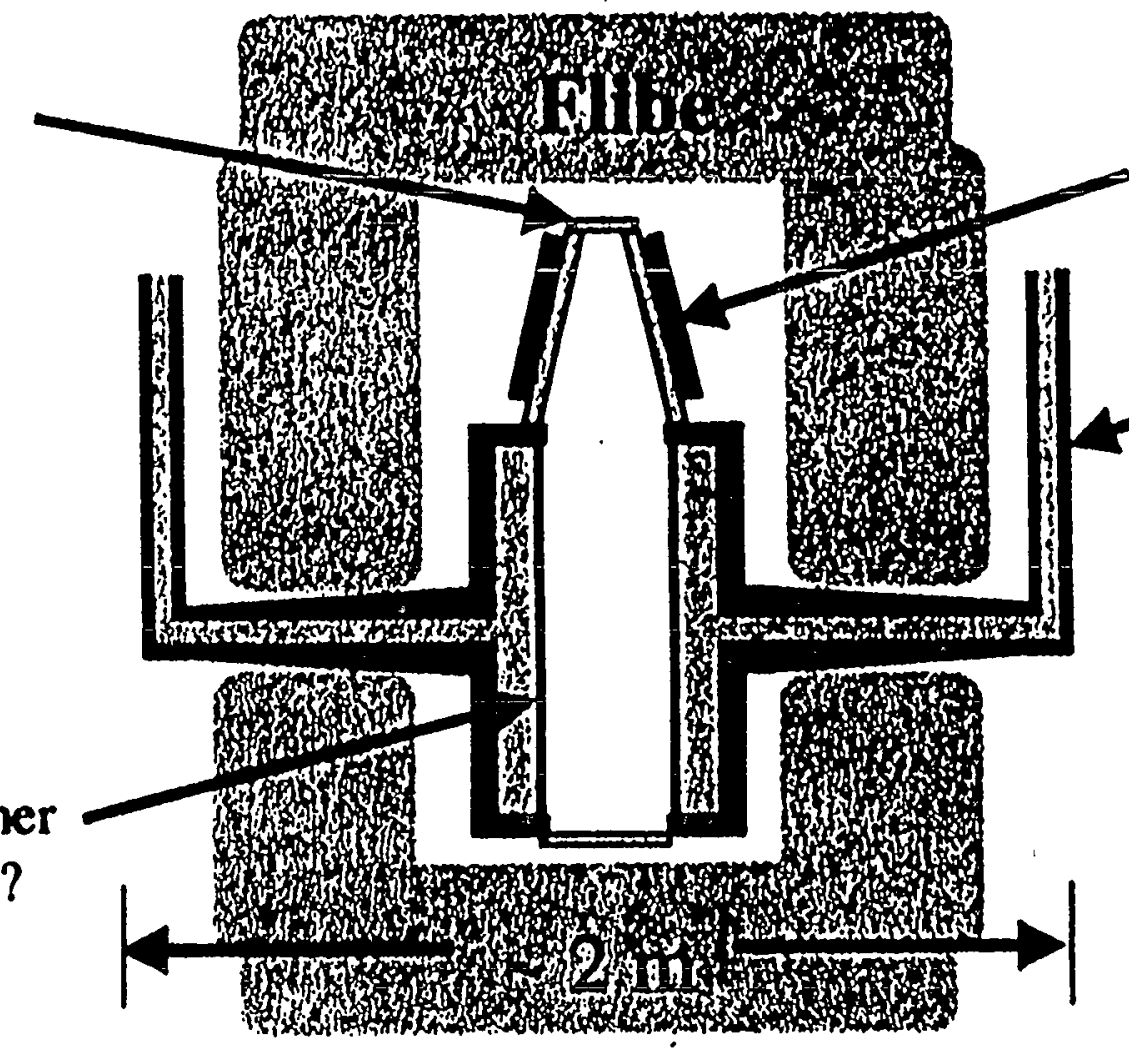

Conical theta pinch Sn motal

Low-precision
current feeds
Sn metal

Thermal hydraulics: Per Peterson, UC Berkeley Advanced manufacturing for low cost: Ron Miller, UCSD 


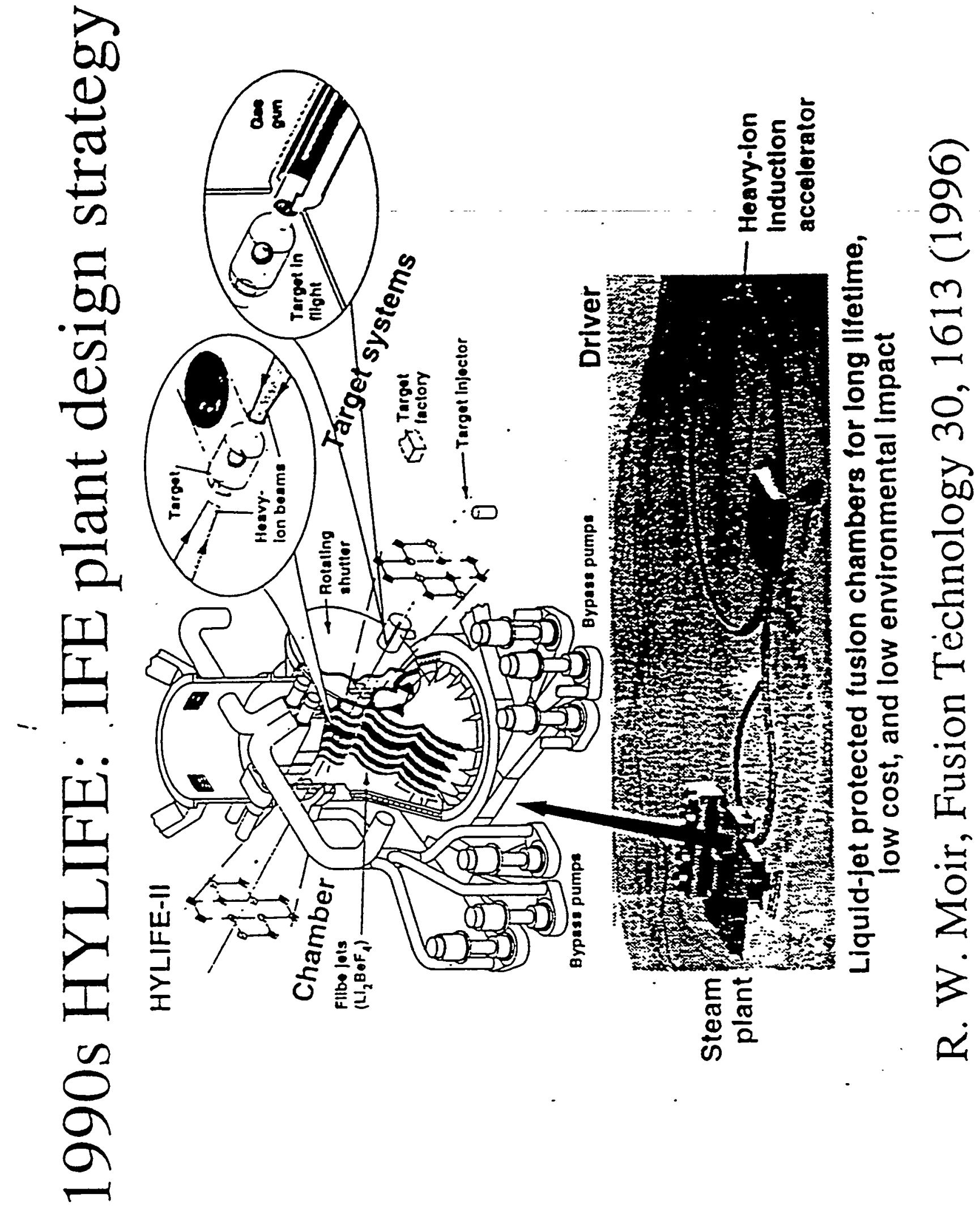




\section{Attractive features of HYLIFE}

- Very low activation achieved using non-flammable, renewable liquid $\mathrm{FLiBe}\left(\mathrm{Li}_{2} \mathrm{BeF}_{4}\right)$ as neutron breeding blanket and coolant

- Upon decommissioning reactor materials qualify for shallow burial disposal

- Neutron sources to develop first wall materials are no longer necessary

- Stainless steel containment vessel predicted to last for plant life of 30 years with less than $100 \mathrm{dpa}$. 


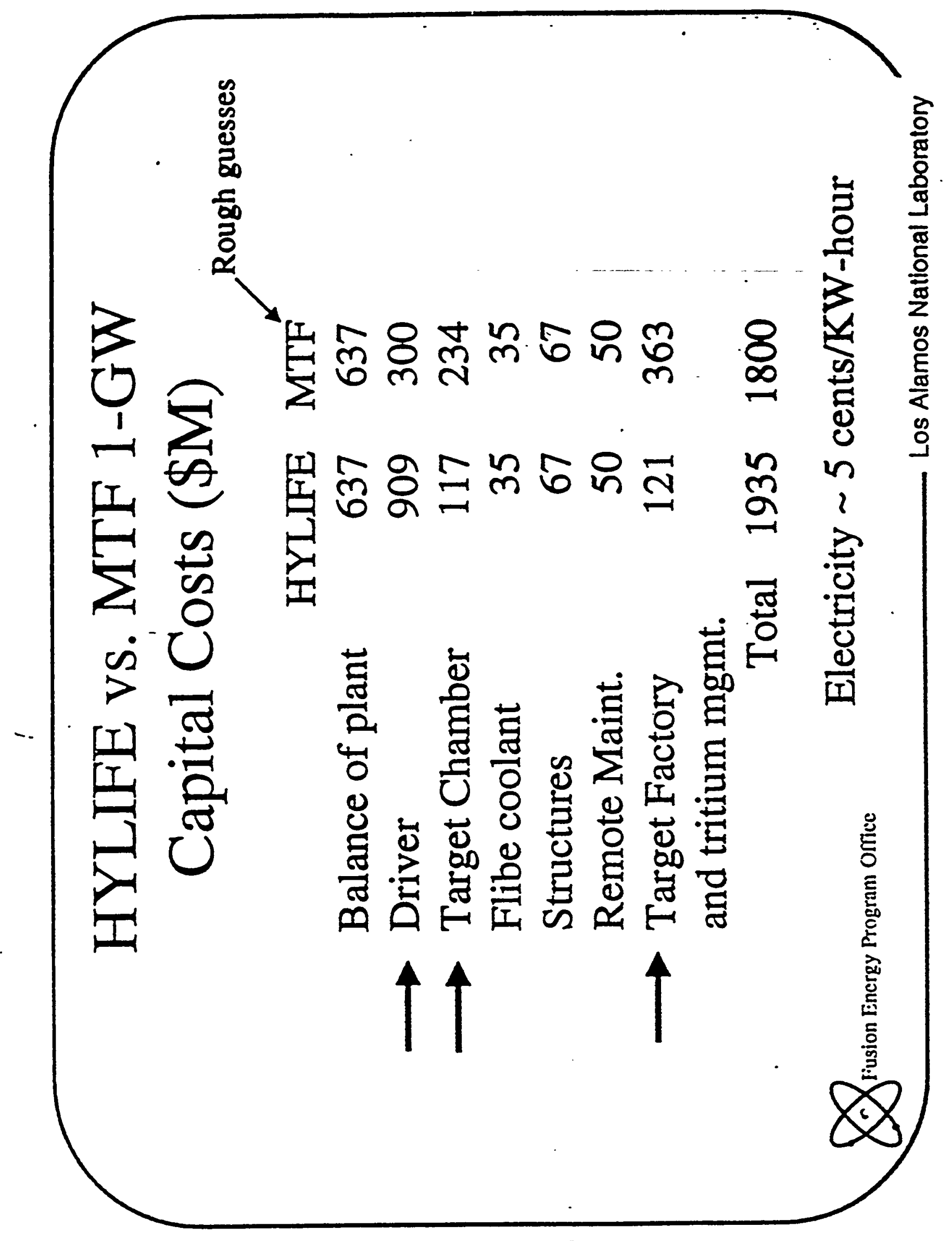




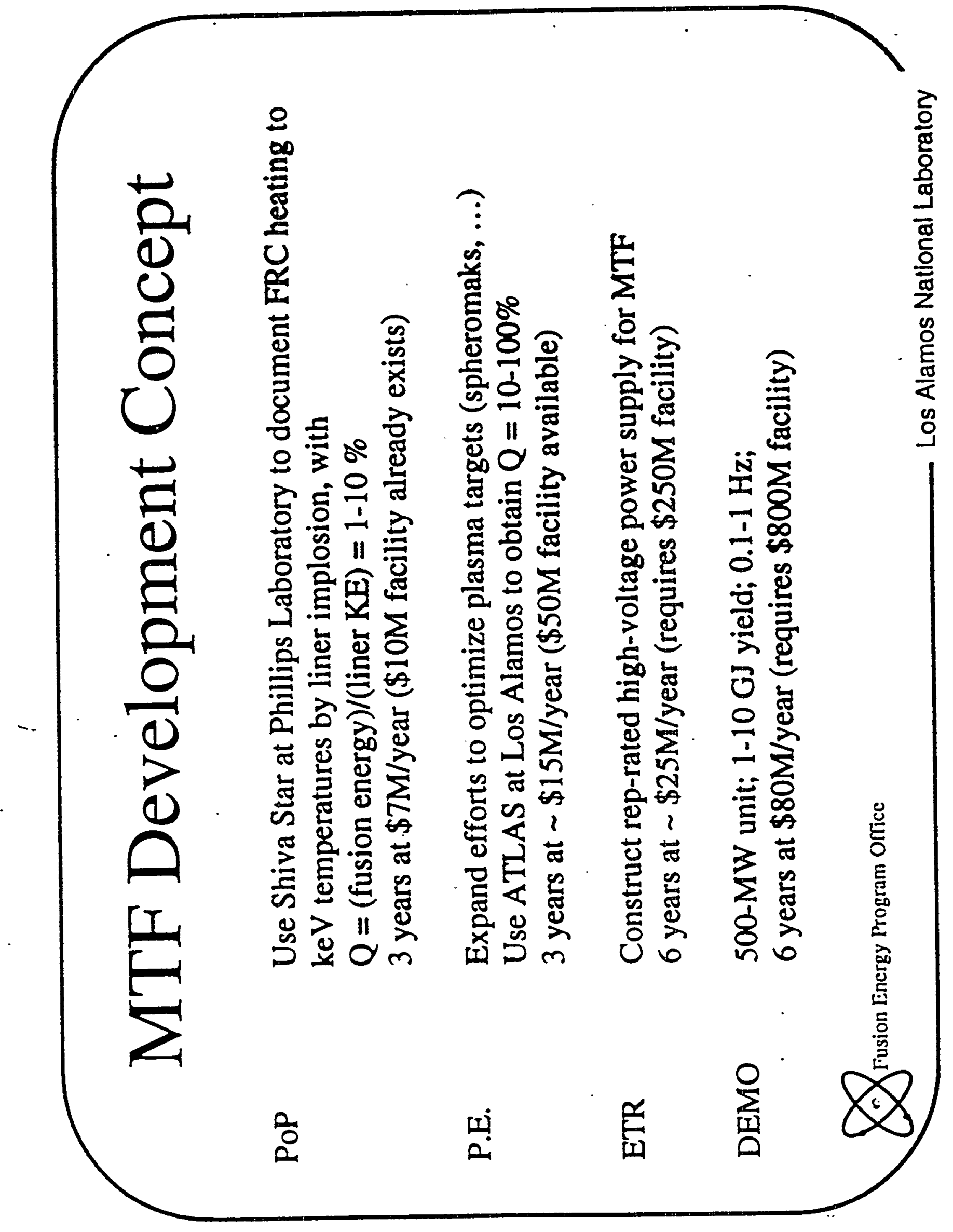




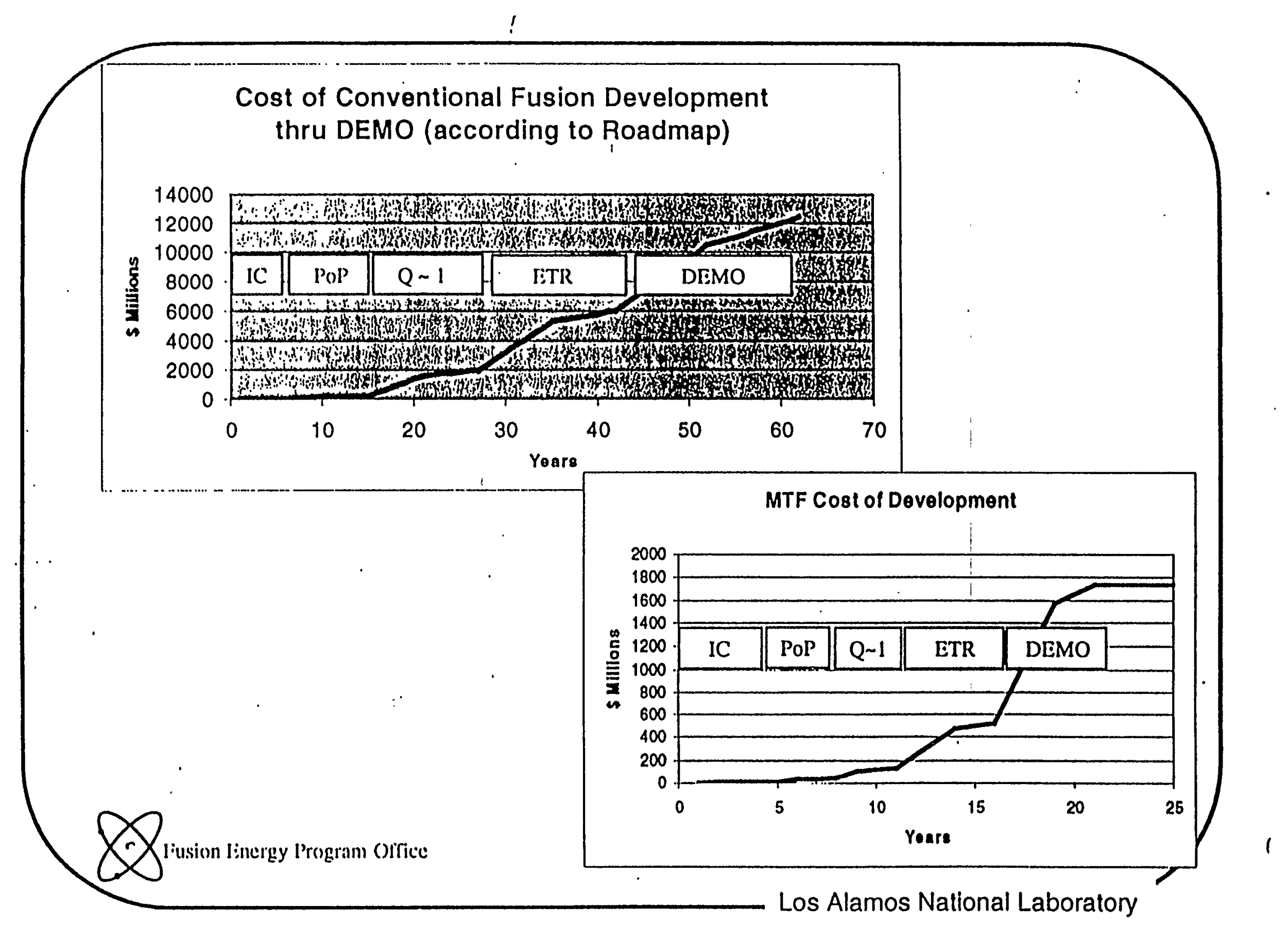




\section{Roadmap to Attractive Fusion Power - A Portiolio Approach -}

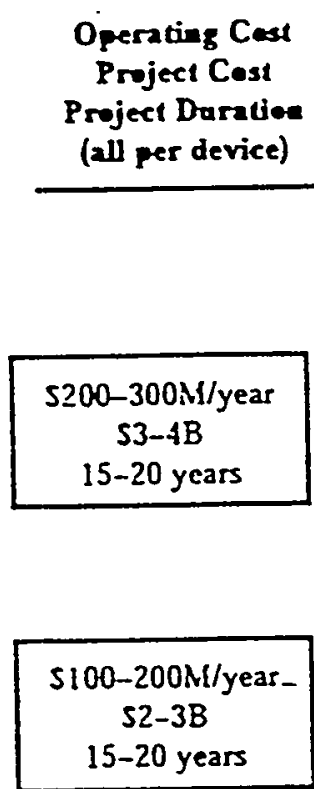

$560-100 \mathrm{M} /$ yeas

50.2-2B

$10-15$ years

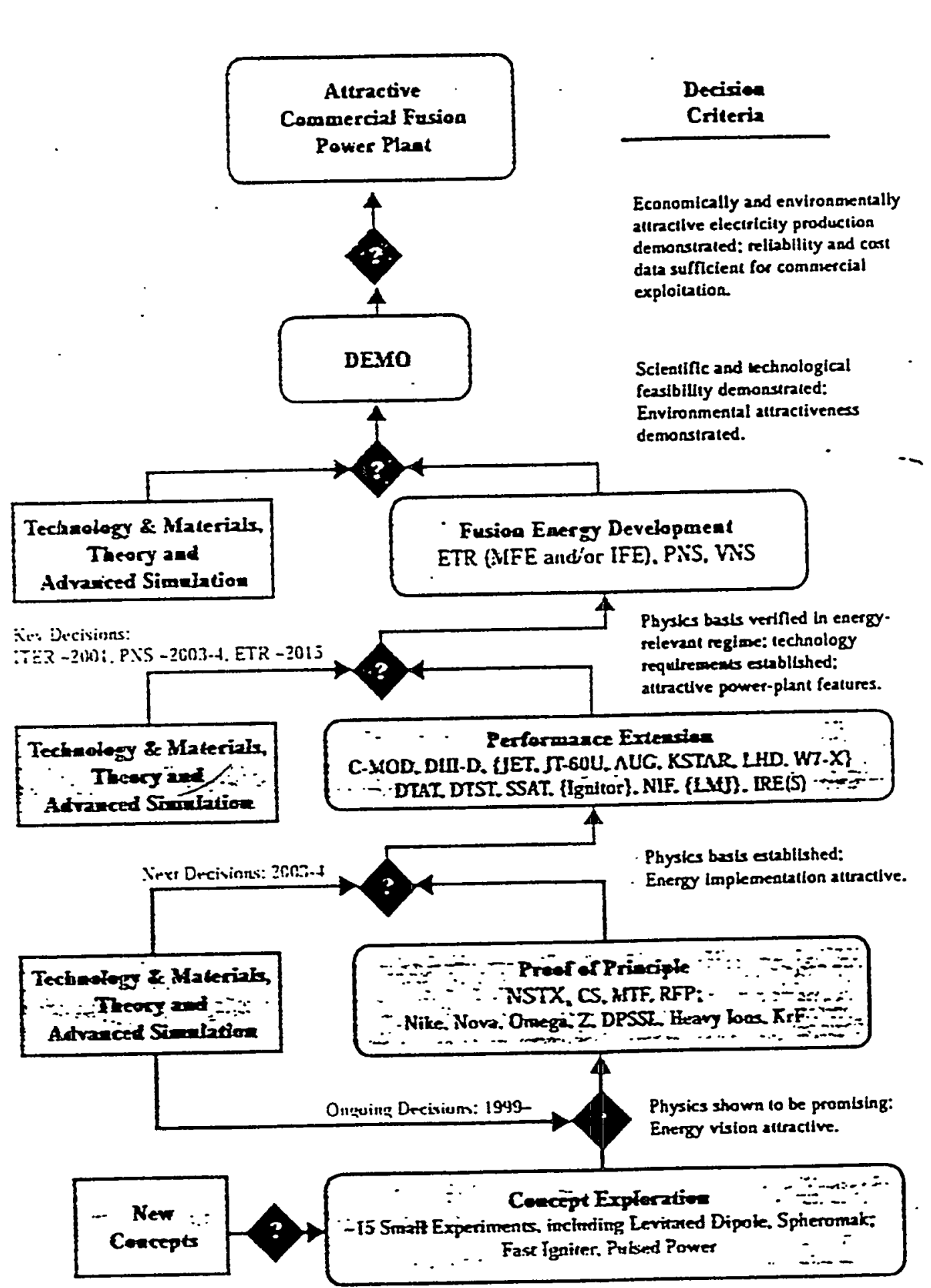

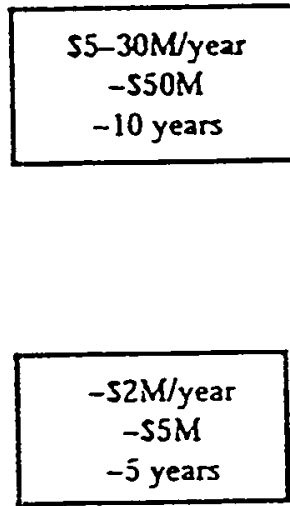

BLLE = Operating or unicr construction. RED TT:ILIC = Examples to be decided. \{Curly brackets $\}=$ Major forelgn facility 


\section{$\underline{\text { Z-pinch }}$}

Advantages

- Compact

- High power density

Applications

- Magnetic fusion $\left(\mathrm{n} \approx 10^{21} \mathrm{~cm}^{-3}, \tau \approx 100 \mathrm{~ns}\right)$

- Radiative Collapse to high density at I $>\mathrm{I}_{\text {Pease-Braginskii }}$

- Intense X-ray source for ICF hohlraum using wire array implosion

Problem

- Stability - MHD unstable

- But Large ion Larmor radius, sheared axial flow or axial magnetic can reduce growth or saturate modes.

- Better approach - implosion of wire arrays 


\section{Past Programme}

- Bare carbon and cryogenic deuterium fibres; found to be unstable in ionising resistive phase

\section{$\underline{\text { Present Programme }}$}

- Physics of wire array implosions

Explosion of single wires $\bullet$ merger $\bullet$ implosion $\bullet$ Rayleigh-Taylor instabilities

- stagnation $\bullet$ X-radiation $\bullet$ nested wires

- Collapse of wire array on to a central fibre $\left(\mathrm{CD}_{2}\right)$

Transfer of current $\bullet$ radiative preheat $\bullet$ stability $\bullet$ tamping $\bullet$ mixing

- Additional axial magnetic liceld

Trapping after wire merger $\bullet$ compression $\bullet$ stability and separation of core fibr

- Cryogenic $\mathrm{D}_{2}$ (fusion and $\mathrm{H}_{2}$ (radiative collapse) central fibre 
$Z$-pinch reactor studies

1. Hartman et al, Nucl. Fusion 17 (1977) 909

2. Hagenson etal, Nucl. Fusion 21 (1981) 1351

3. Robsen, $1^{\text {s5 }}$ Conf. on DZP (1988)

4. Imperial College DZP. reactor seoping study

- Bolton etal, $11^{\text {th }}$ Int. conf. (IAEA, 1986)3,367

- Halies + Walker, Nael. Energy (1987) ख6,361

- Marines ef 1 , $11^{*}$ Int.cont. (14teA, 1986) 3, 573

- Power + Boltox, 6 theEe Pulsed Power (1987)

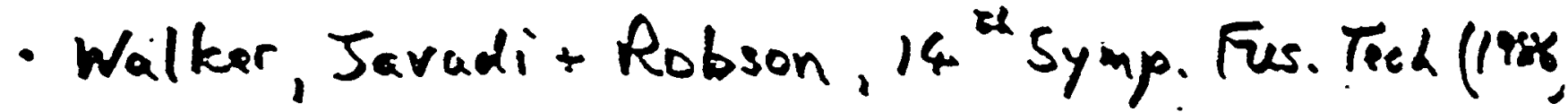

- Walker + Javadi , Nucl. Fusion Eas +D. 5 (1987)261

- Walker, Nuel. Energy

- Bulton et al, Fusion Eng.+ Design 10 (1989) 9 


\section{Deuterium Fibre Results}

14 shots have been fired into $D_{2}$ fibres with diameters around $80 \mu \mathrm{m}$ using $50-60 \mathrm{kV}$ charge $\left(I_{\max } \sim 0.85-1.05 \mathrm{M \Lambda}\right)$ with an average neutron yicld of $5 \times 10^{9}$, mostly beam-target in origin.
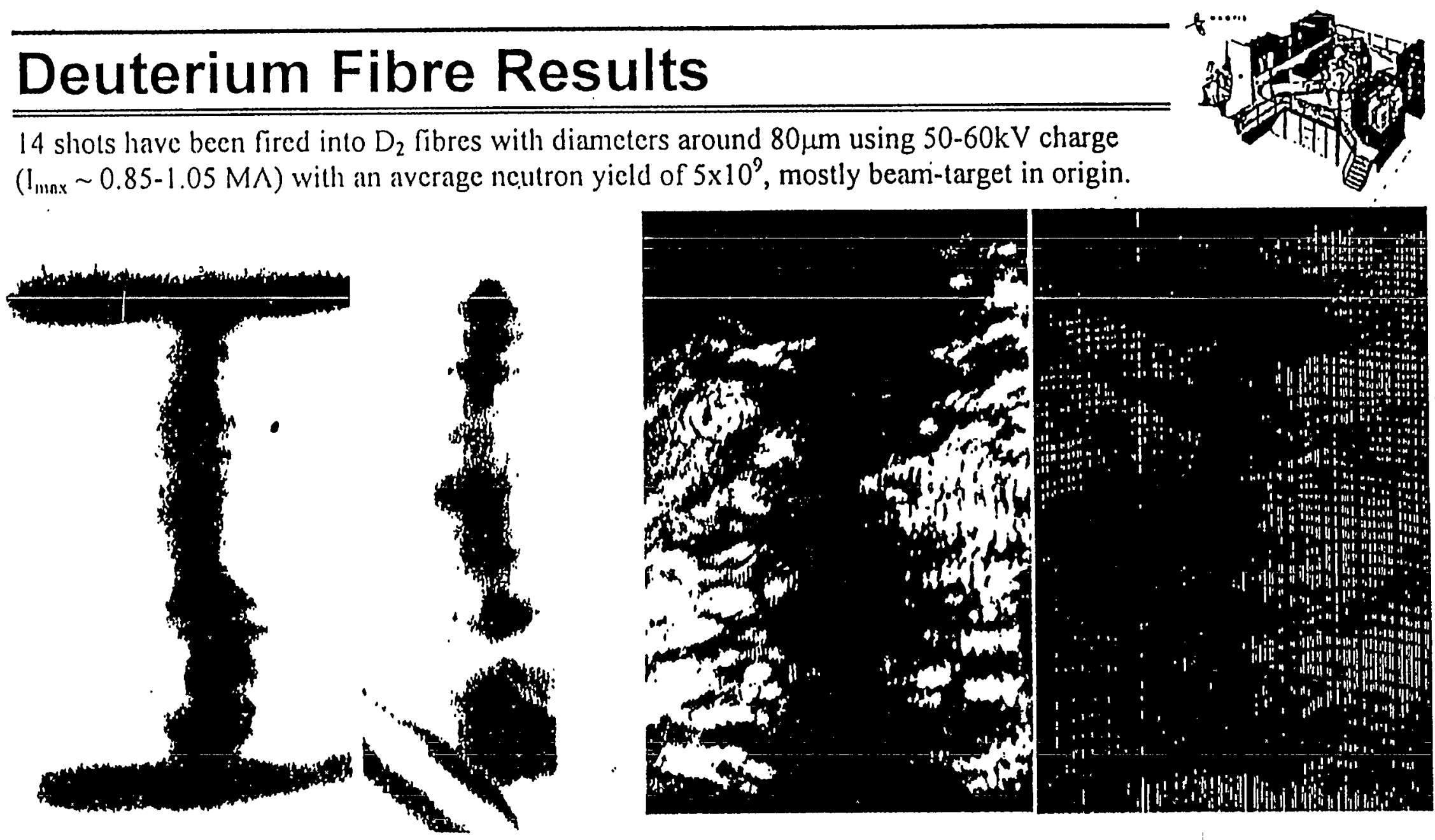

Ins Gated optical image 2ns Gated X-ray image 300 ps Schlieren 300 psp Interferogram

IOP Conference on Plasma Physics, Leeds, 25 ${ }^{\text {th }}$ March 1997. 
$\because$ Reactor advantages

1. Small and scalable.

2. Lithium acts as $1^{\text {st wall }}$, return conductor, energy transfer, :.

3. No impurities. ( $\left.c<\tau_{\text {impurities }}\right)$

4. No anomalous thermal insulation required

5. No coil or superconducting material

6. High $\beta$. $B \sim 50 M G$.

7. No secondary heating. (laser is small)

8. Stability (a) $a_{i} \sim a$

(b) helical minimum energy state

a. Matrix array of $Z$-pinches in moderator - iefficient use of blanket

10. Range of $\alpha$-particle $\simeq$ Length of pinch - can choose to have some burn

11. In absence of $\alpha$-particle heating, $Q$ $\sim 100$ possible

12. Highly compressed state possible by radiative collapse 


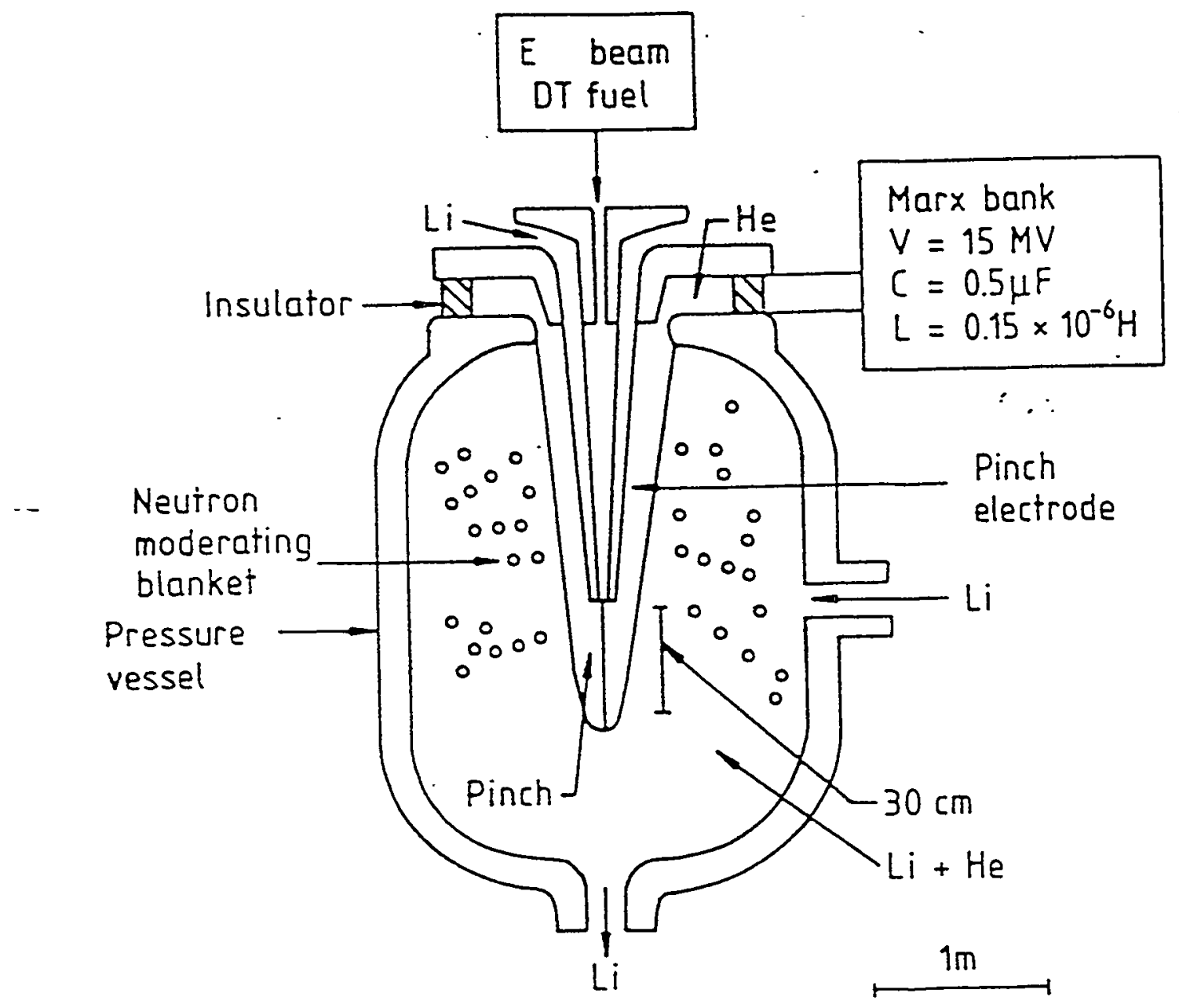

Fig.5.1.1 The conceptua! schematic reactor design of Hartman e: al 


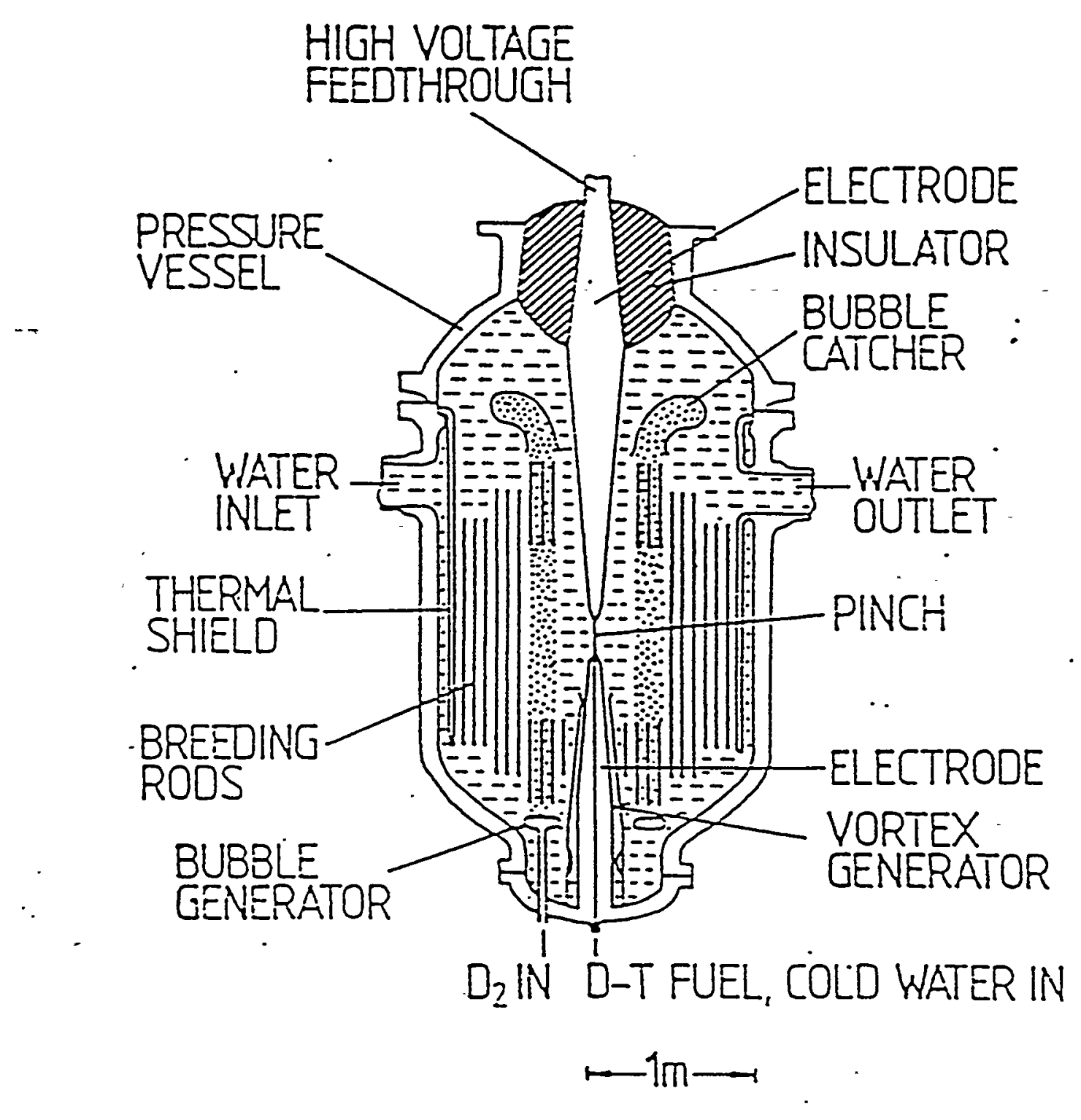

Fig 5a. Waier-cosled DZP recctor proposed by Robson ${ }^{1}$ 


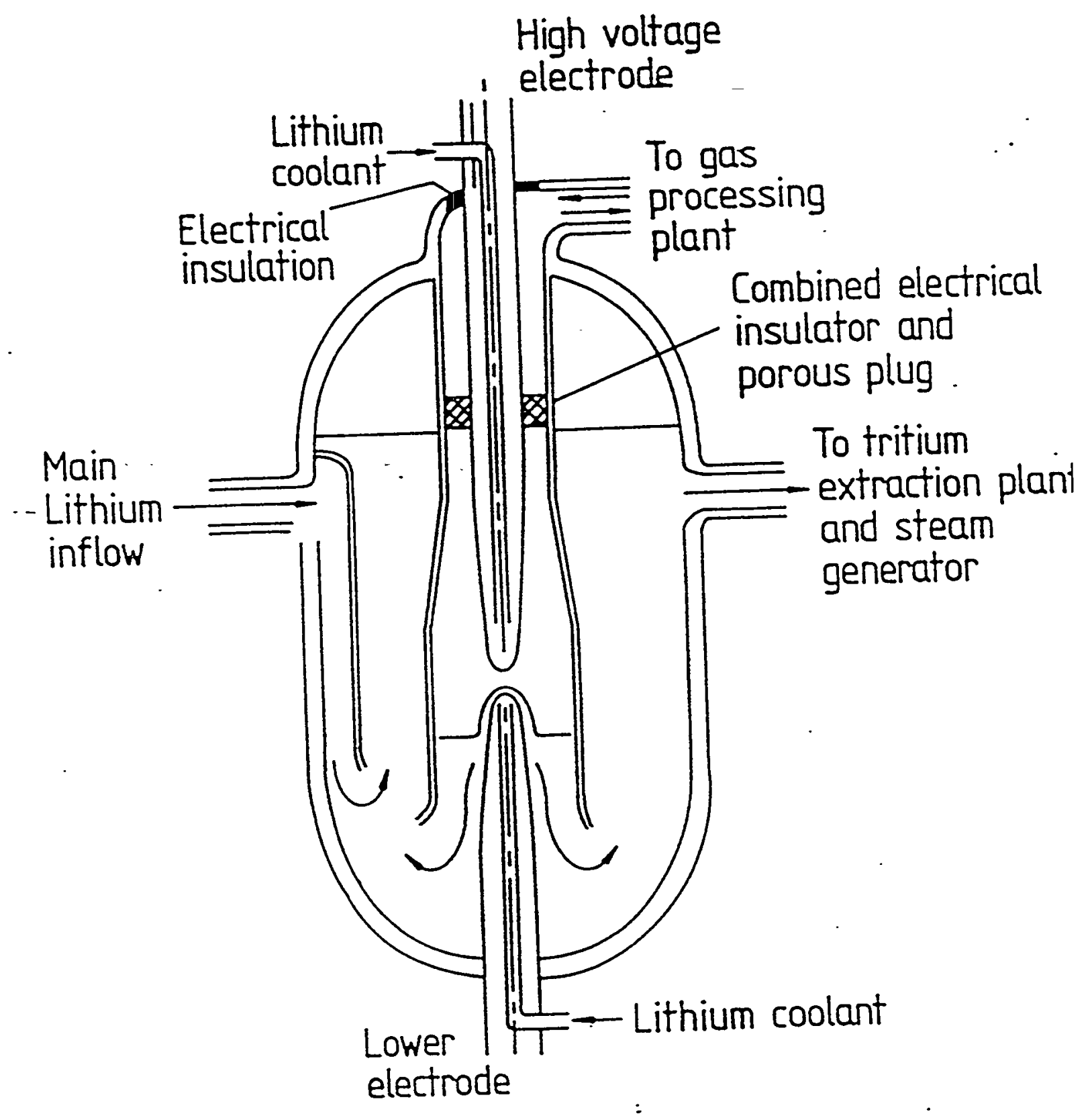

Fig 1b. DZP reactor concept with expendable electrodes and gas/liquid separator. 


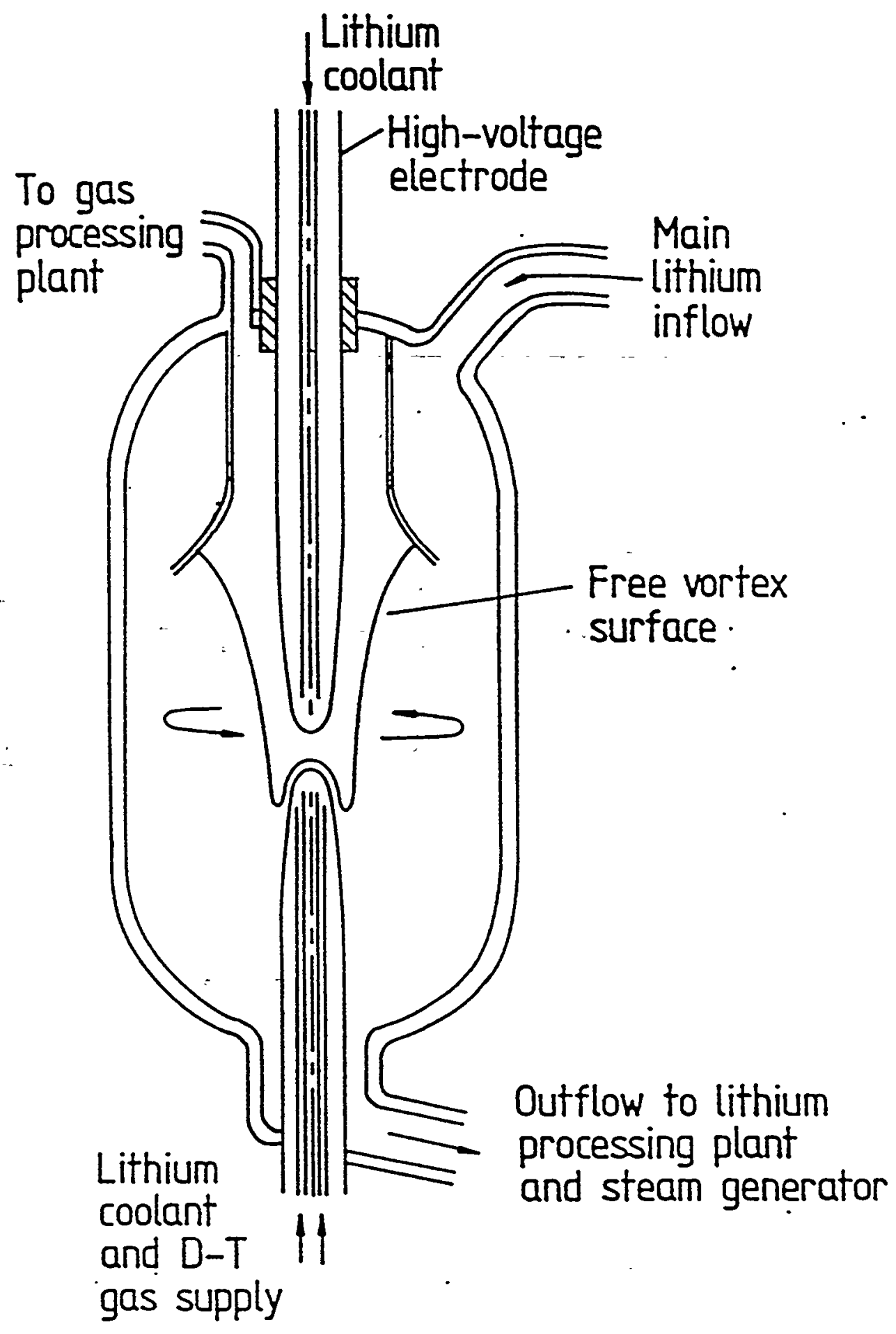

Fig 1c. DZP reactor concept with expendable electrodes and vortex surface gas/liquid interface. 


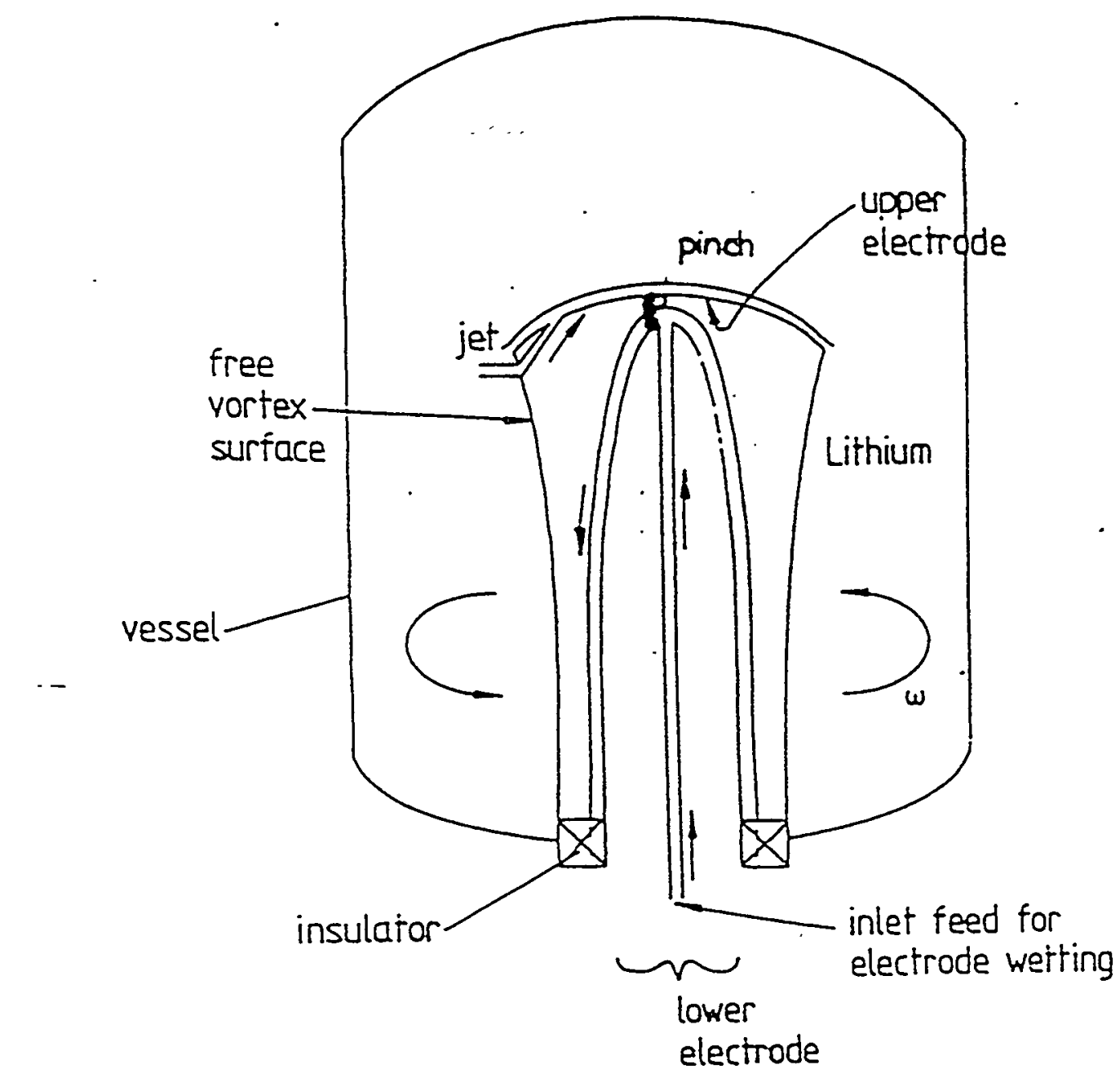

Fig. 1. Schematic outline design of conceptual DZP reactor. 
VOLTAGES

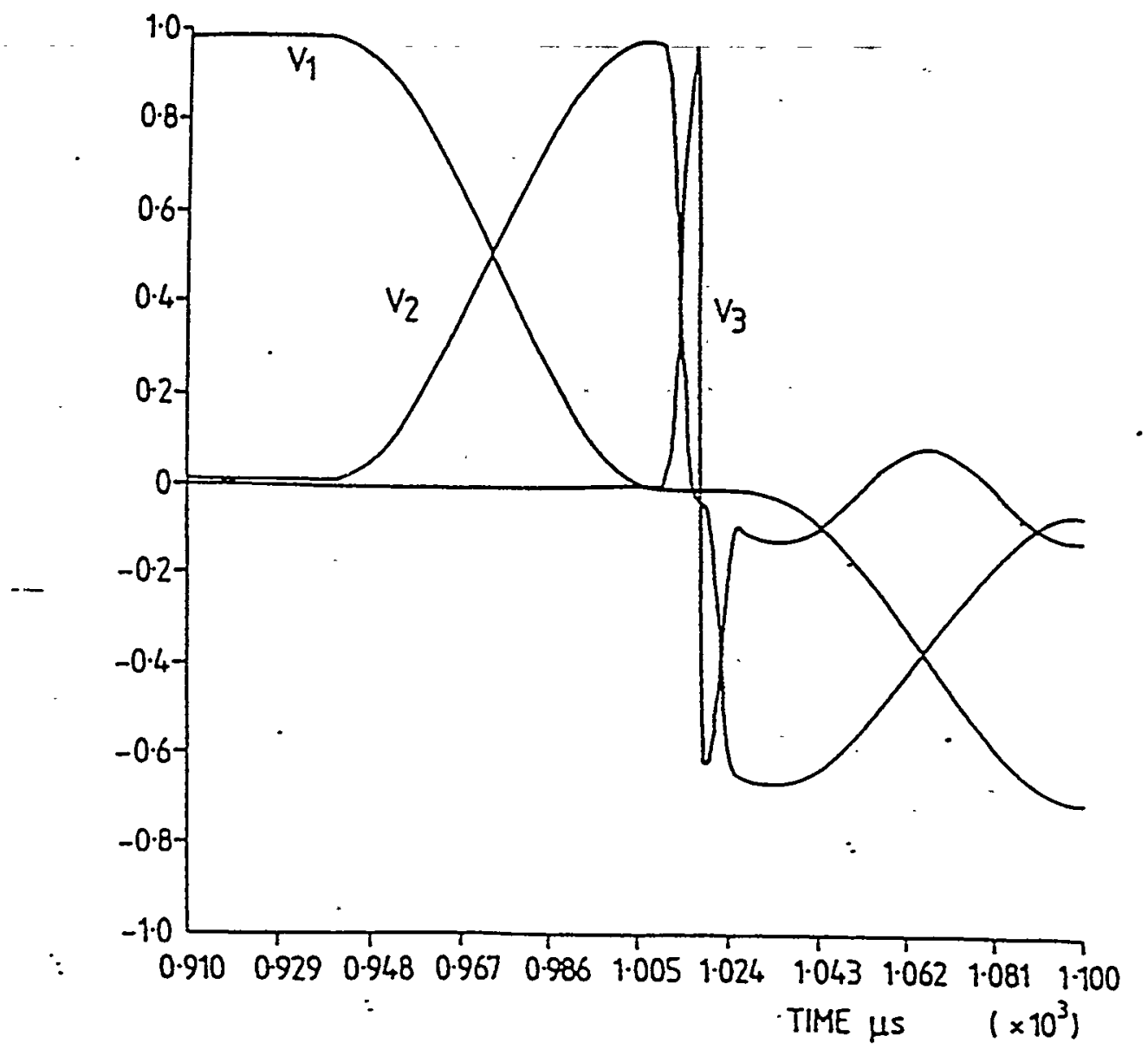

Fig. 3. Voltage waveforms for pulse compression simulation. Note voltage reversal at the load $\left(V_{3}\right)$. The maximum voltage $(=1)$ corresponds to $600 \mathrm{kV}$. 


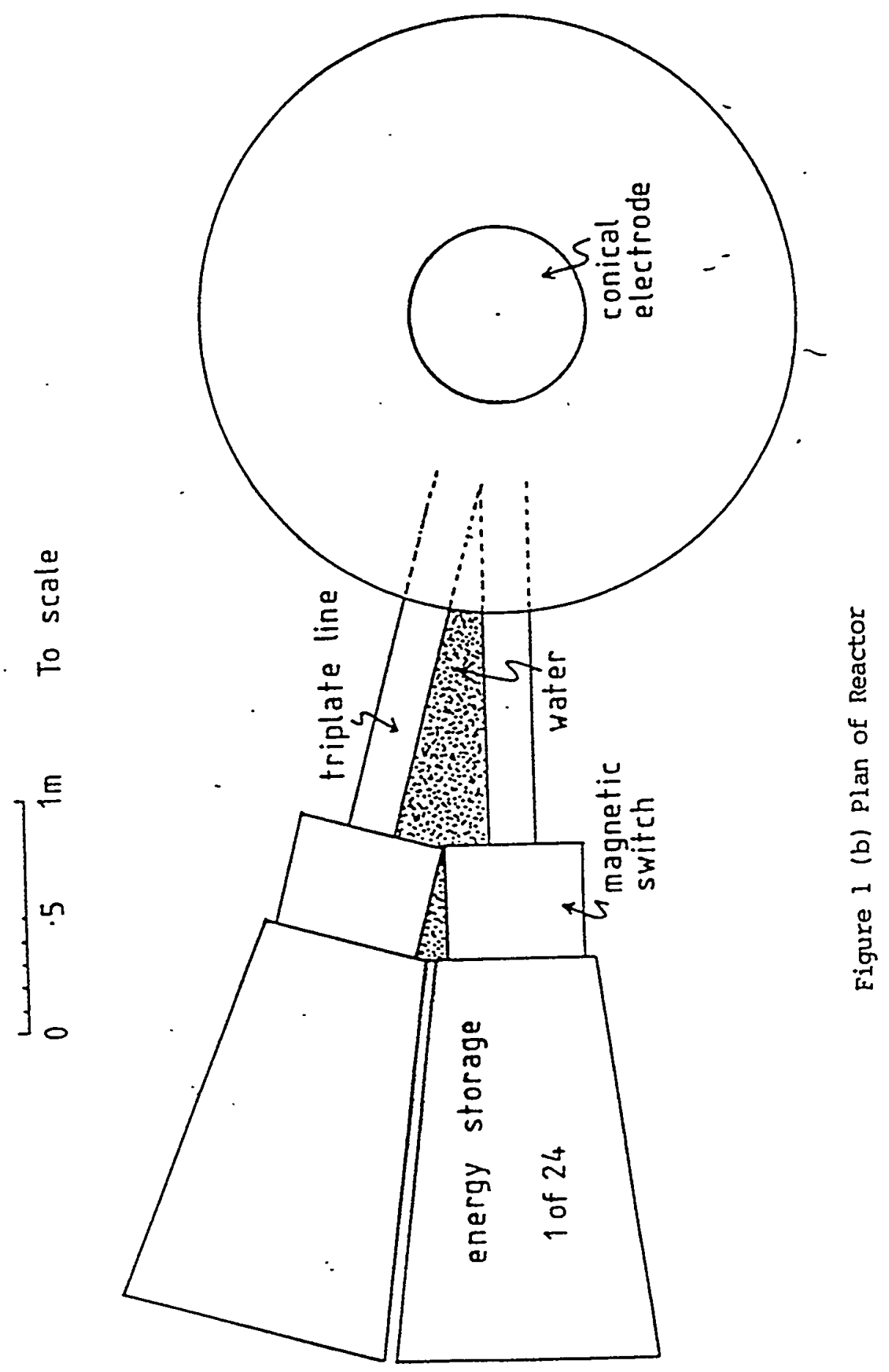




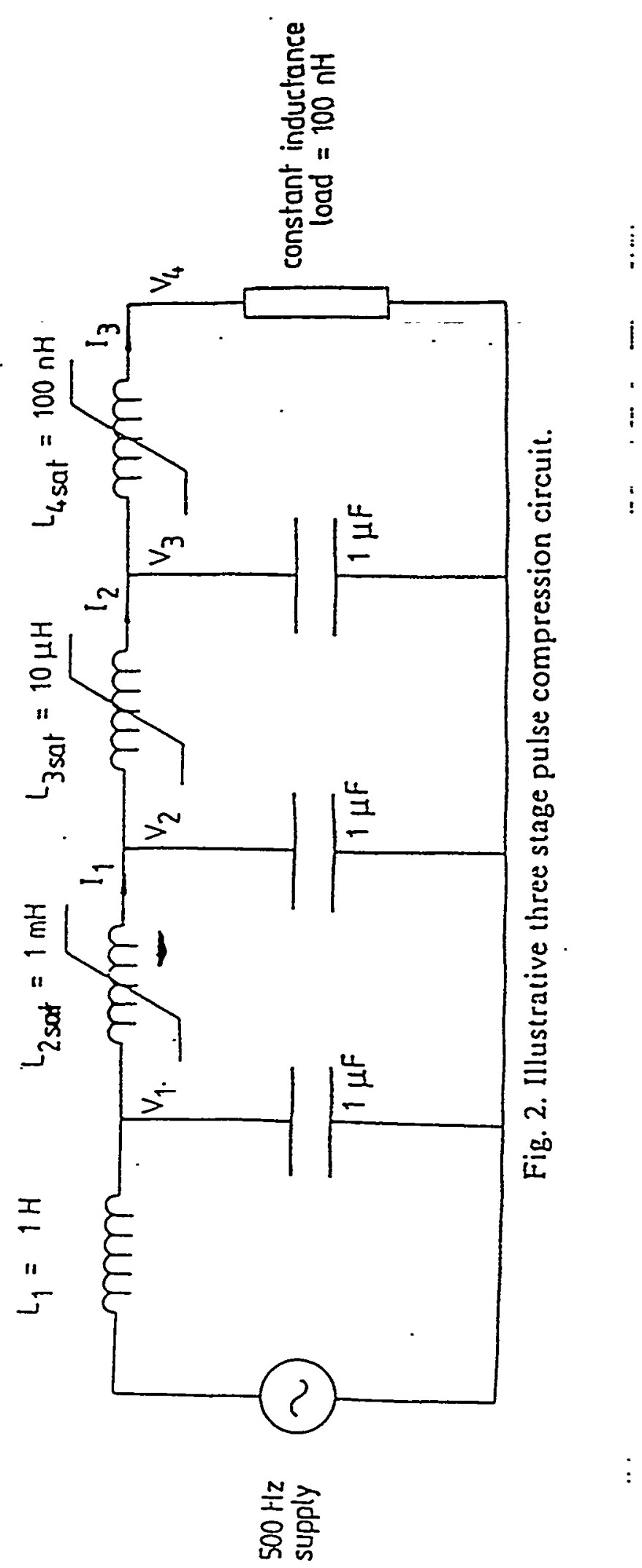




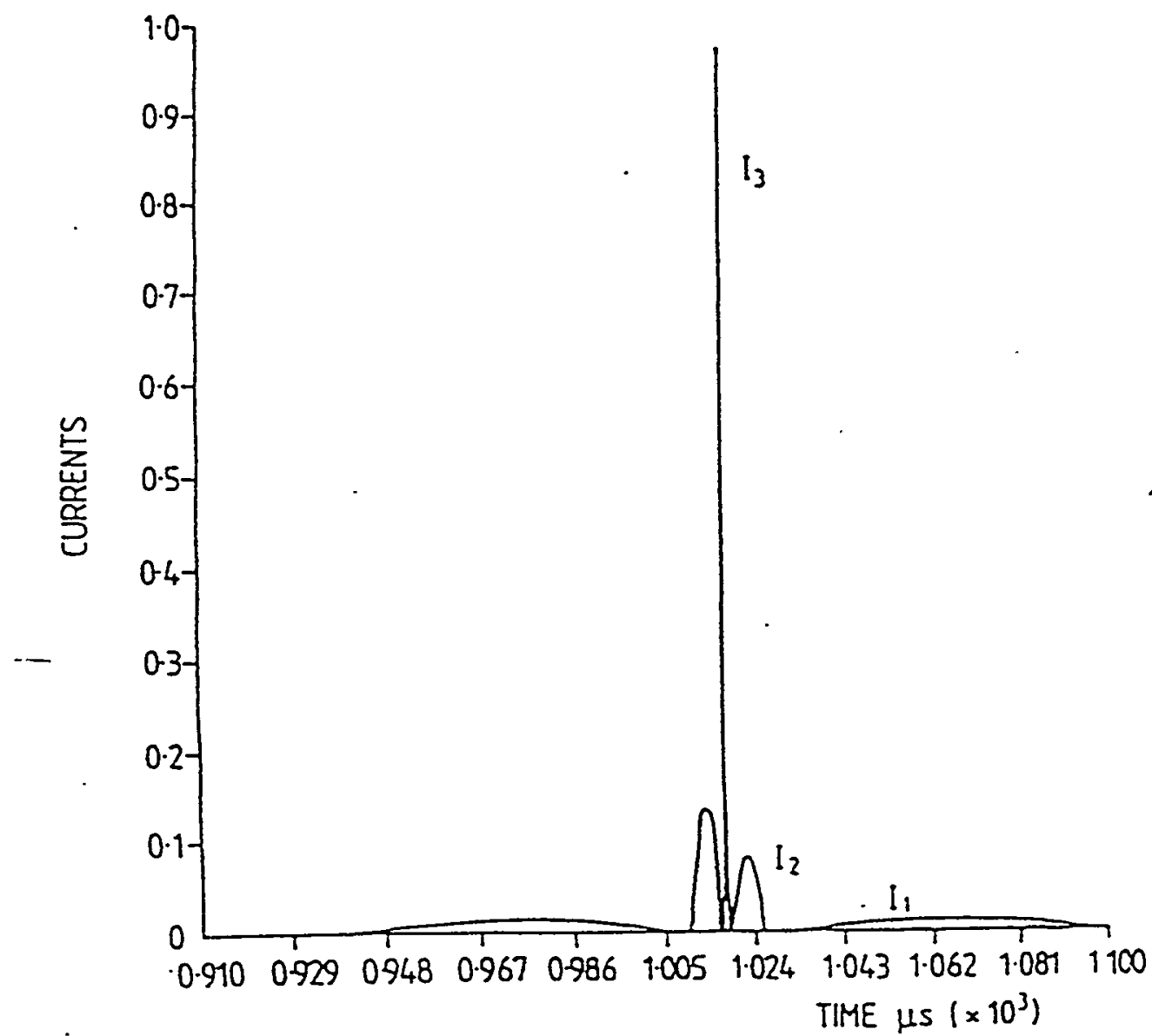

Fig. 4. Corresponding current waveform to fig. 3. Note the asymmetry in the incident and reflected current pulses due to energy dissipation in the load. The maximum current $(=1)$ corresponds tó 1.3 MA. Approximately half the energy is recovered into the first capacitor. 


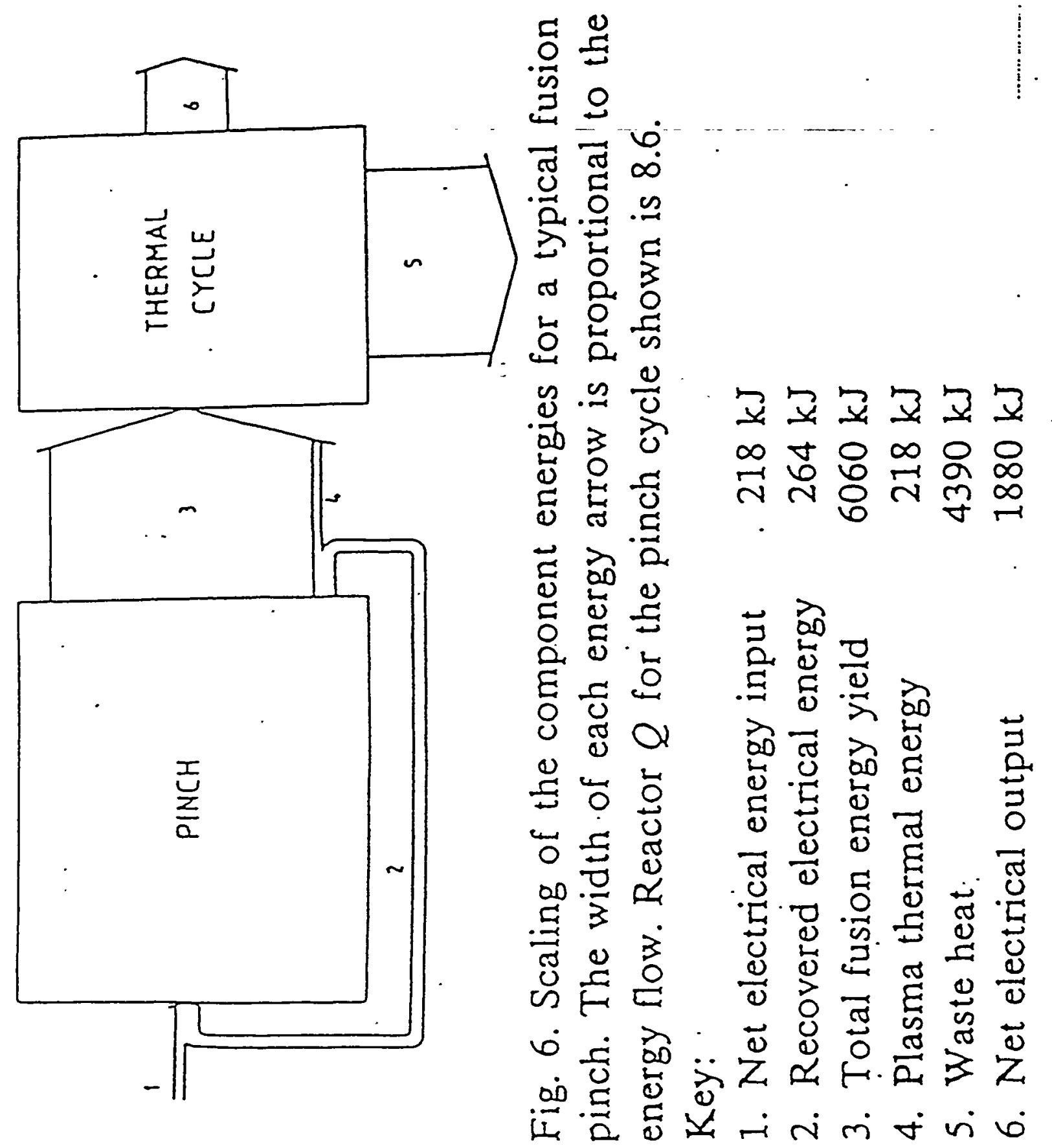



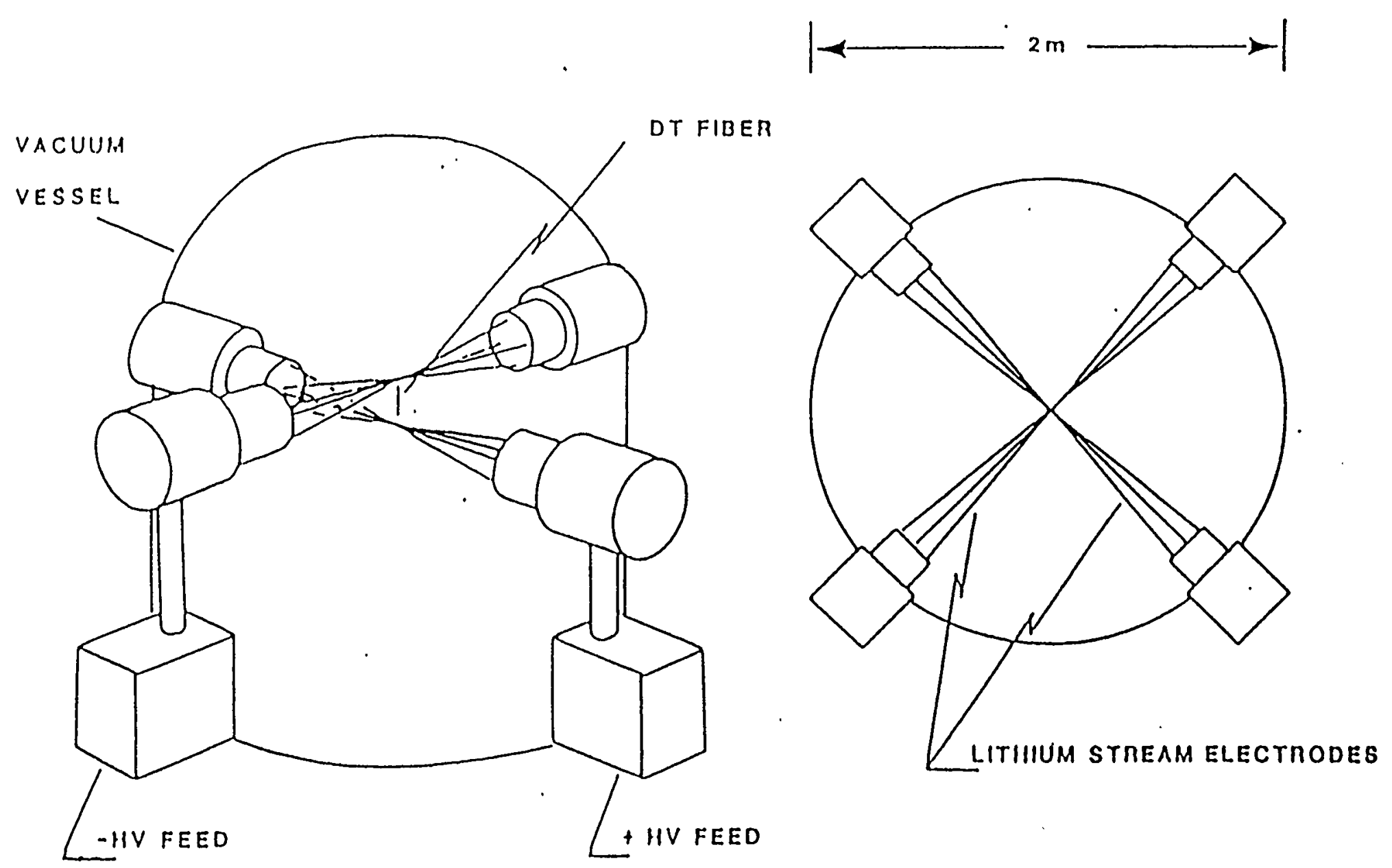

Fig. 6. Electrode arrangement in DZP reactor. 


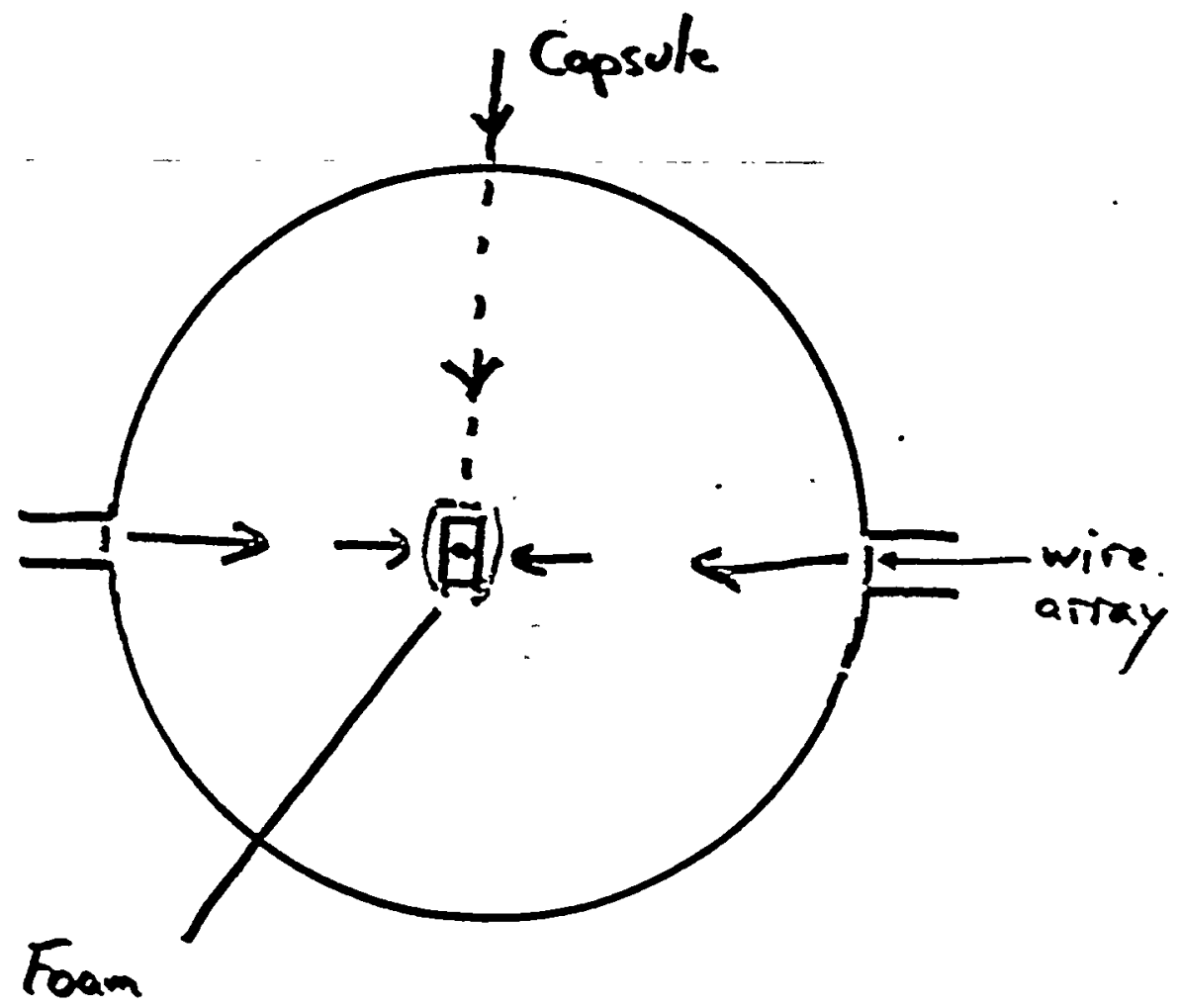

(Av costed)

+ capsule

Divergence of coasting wits plasma

$$
\begin{aligned}
2 \theta & \simeq \int\left(\frac{\text { Temperature }}{\text { Directed K.E. }}\right)=J\left(\frac{35 \mathrm{~V}}{70 \mathrm{keV}}\right) \\
& =\frac{J\left(\frac{1}{2000}\right)=0.02 .}{\mathrm{Im}} 1 \mathrm{~cm} \quad 0
\end{aligned}
$$




\section{PROPOSED DESIGNS FOR Z-PINCH FOR ENERGY MUST CONSIDER THE CRYOGENIC TARGET REQUIREMENTS}

- DT Ice

- Layer Thickness, Uniformity, Surface Roughness

- Temperature

- Capsule

- Material (Be, $\mathrm{CH}$, Polyimide)

- Wall Thickness, Uniformity, Sphericity, Surface Roughness

- Pulse Shaping Cylinder

- Foam

- Helium Gas and Capsule Support

- Hohlraum

- High Z Material (Gold)

- Structural Support (Plastic) 
- DT Filling

- Permeation Filling

- Bond Hemispheres in DT Environment

- Drill, Fill, and Plug

- Cooling, Layering, and Characterization

- Cool to 18K and Remove Excess DT

- Controlled Isothermal Environment

- Sampling and Verification Methods

- Injection into Target Chamber

- Acceleration Forces on Target Components

- Heating from Thermal Radiation

- Heating from Chamber Gases

- Chamber Clearing

- All This Has to be Done Repetitively and Economically!

- Shot Rate (Several per second?)

- Target Cost (<20 cents/target?) 


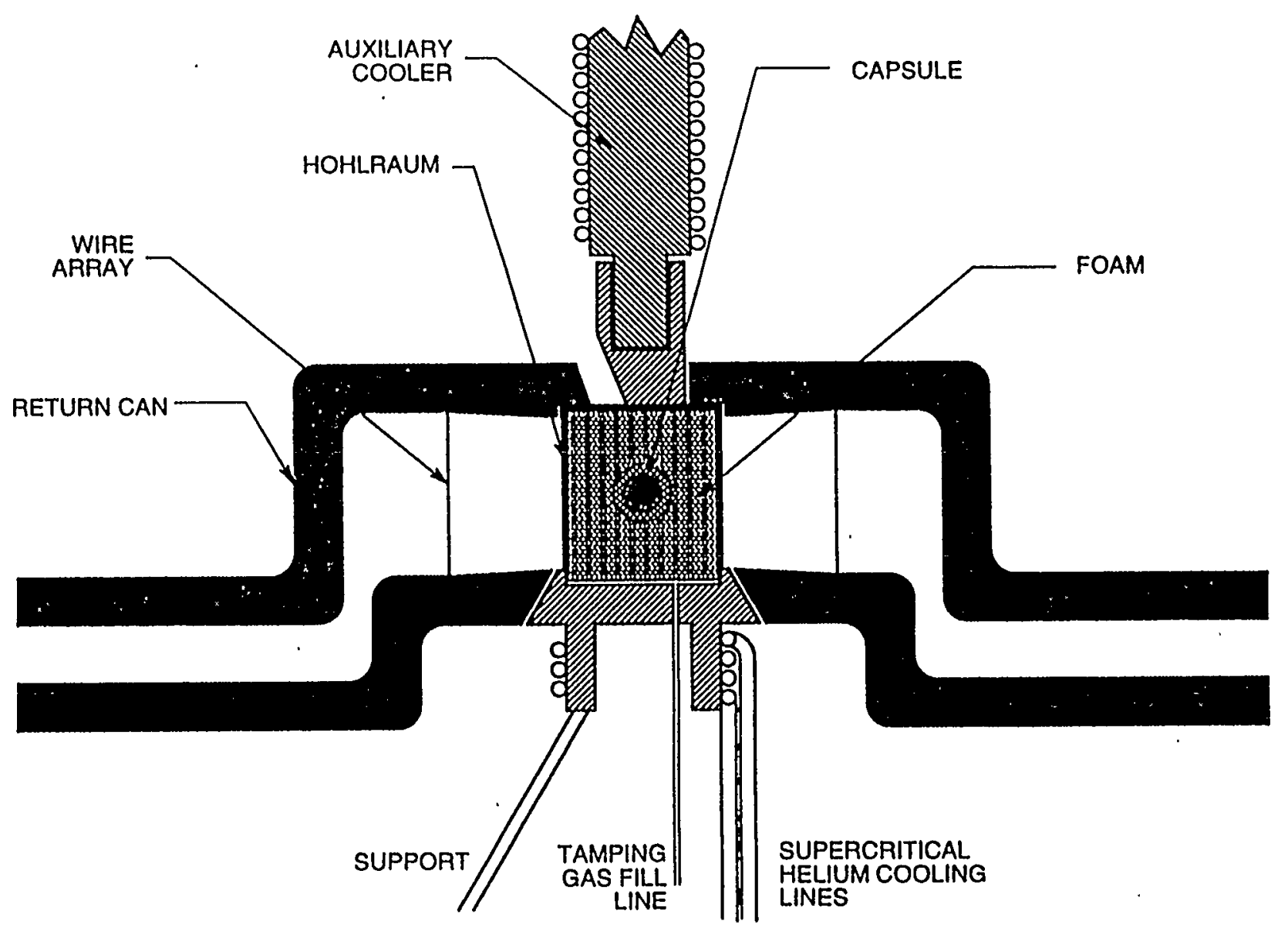

DYNAMIC HOHLRAUM

(BOTTOM INSERTION) 


\section{SOME COMMENTS ON TARGET PHYSICS}

I. R. Lindemuth, Project Leader for Pulsed Power Physics (presenter)

Richard E. Siemon, Program Manager for Fusion Energy

Los Alamos National Laboratory

Los Alamos NM USA

Presented at Z-Pinch IFE Workshop, Sandia National Laboratories, Albuquerque NM, April 27-28,1999. 


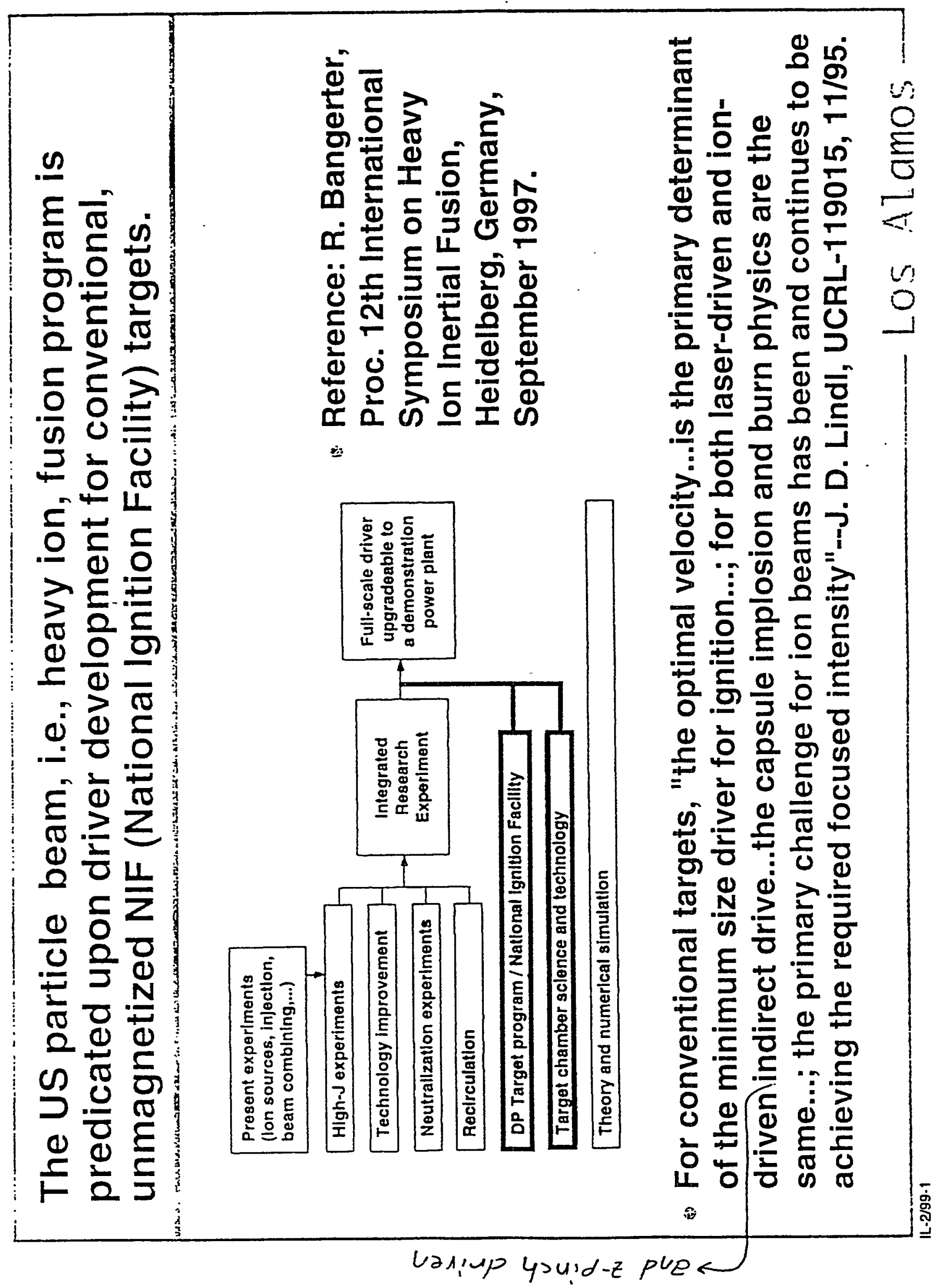


The minimum fusion system size, plasma energy, and heating requirements can be estimated by a transport diffusion coefficient [e.g., thermal conduction, $D=K /(3 n k)]$, the Lawson criterion, geometric factors, and the plasma density.

$$
\begin{aligned}
& \frac{\partial \mathrm{Q}}{\partial t}=-\nabla \bullet(D \nabla \mathrm{Q}) ; \quad \mathrm{Q}=T_{i}, n, B, \text { etc. } \\
& \oint_{\mathrm{V}} d V \Rightarrow \frac{\mathrm{Q} V}{\tau}=-S D \nabla \mathrm{Q} ; \quad V=\text { volume, } S=\text { surface area } \\
& \nabla \mathrm{Q}=-\frac{\mathrm{Q}}{\alpha a} ; \quad \quad V=\varepsilon a^{3} ; \quad \frac{V}{S}=\gamma a ; \quad M=n m_{i} \varepsilon a^{3}
\end{aligned}
$$

where $a=$ characteristic dimension; $\alpha, \varepsilon, \gamma$ geometry dependent

$$
\begin{array}{lll}
\tau \approx \tau_{\text {burn }} \approx \tau_{\text {heat }} ; & \tau_{\text {burn }}=\frac{L}{n} ; & L=(n \tau)_{\text {Lawson }} \\
a^{2}=\frac{D L}{\alpha m} ; & E_{\text {plas }}=3 n k T \varepsilon a^{3} ; & P_{\text {hear }}=\frac{E_{\text {plas }} n}{L} .
\end{array}
$$

Intensity $I_{\text {heat }}=P_{\text {heat }} / S \quad$ For pdV heating, Implosion velocity $=\frac{I_{\text {heat }}^{0}}{2 n k \mathrm{~T}}=\frac{3 \gamma a}{2 L} n$ 
Simple scaling relations are accurate over the full density range of controlled fusion research.

- At fixed T (e.g., $10 \mathrm{keV}$ ): $a=c_{1} K^{1 / 2} / n \quad p=c_{2} n \quad M=c_{3} K^{3 / 2} / n^{2}$

$$
E_{\text {plas }}=c_{4} K^{3 / 2} / n^{2} \quad P_{\text {heat }}=c_{5} K^{3 / 2} / n \quad I_{\text {heat }}=c_{6} K^{1 / 2} n \quad v=c_{7} K^{1 / 2}
$$

- The energy and power requirements for conventional, unmagnetized targets force ICF to operate at high density $\left(\right.$ e.g., $\left.>10^{25} / \mathrm{cm}^{3}\right)$, leading to extremely high intensity on target.

- Highly efficient, energy-rich z-pinch radiation generators substantially increase the chances that fusion can be obtained with conventional targets.

- A "steady-state" constraint forces MFE to operate at low density (e.g., $10^{14} / \mathrm{cm}^{3}$ ); system size, energy are practical only if $K$ is reduced orders of magnitude by a magnetic field.

- The reduction of $K$ by a magnetic field makes target fusion more feasible. 
Using $p d V$ compressional heating by an imploding wall, i.e., pusher, to access the intermediate density $\left(10^{20} / \mathrm{cm}^{3}\right)$ regime is now referred to as Magnetized Target Fusion (MTF).

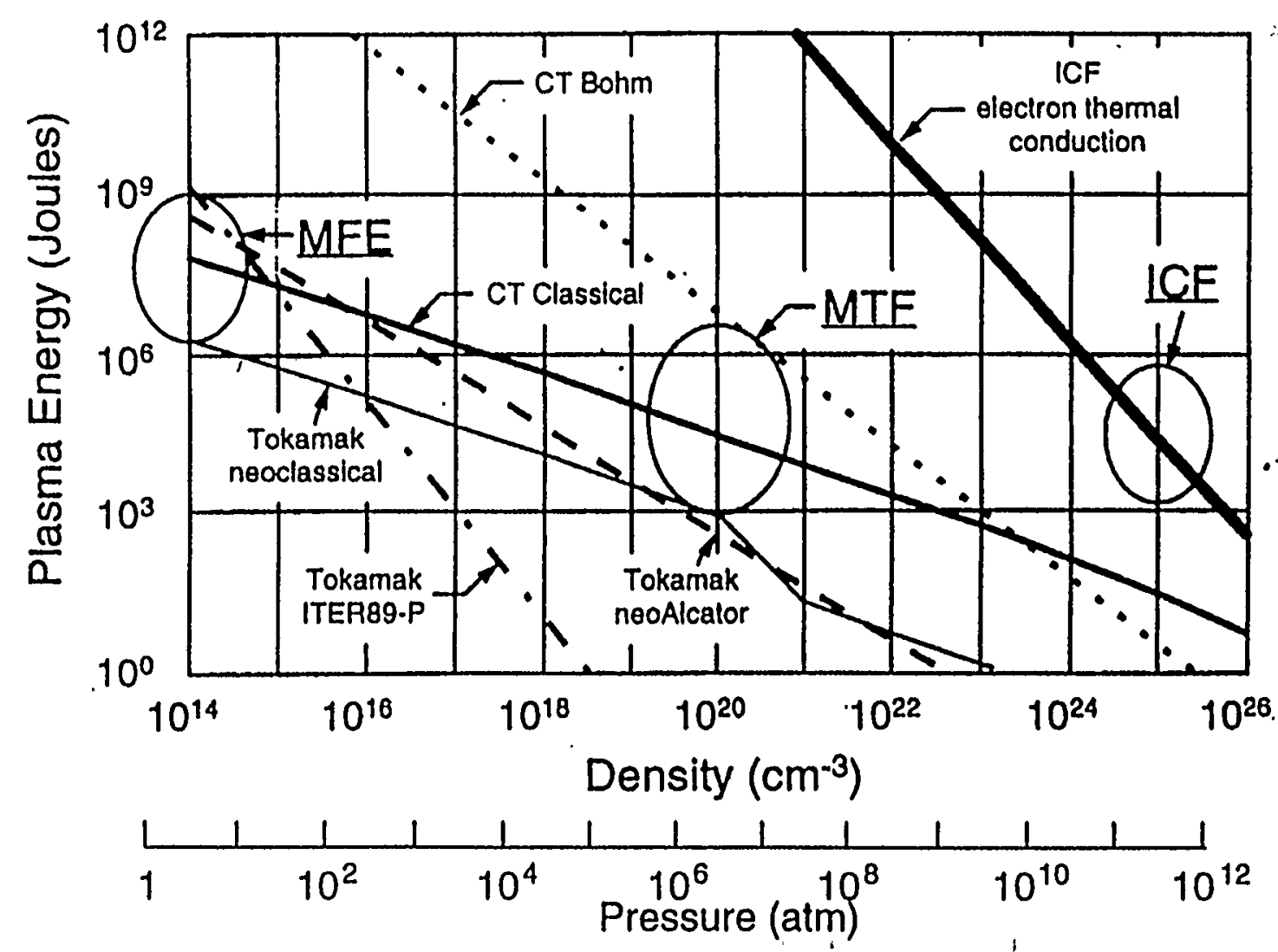

- By combining magnetization with a pulsed system, MTF leads to smaller size and lower energy than MFE and to lower heating and intensity than ICF. 
The reduced driver requirements of magnetized targets potentially can reduce the cost of heavy ion fusion.

(- If the focusing required for conventional targets cannot be obtained, heavy ion fusion may be feasible only with magnetized targets.

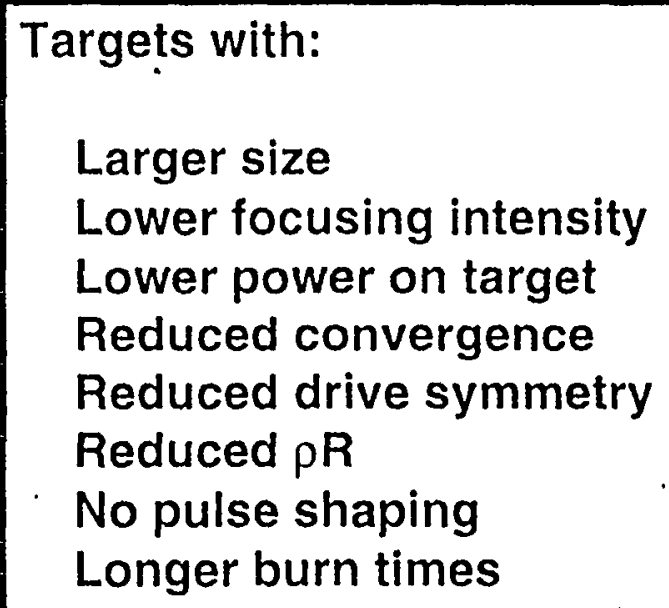

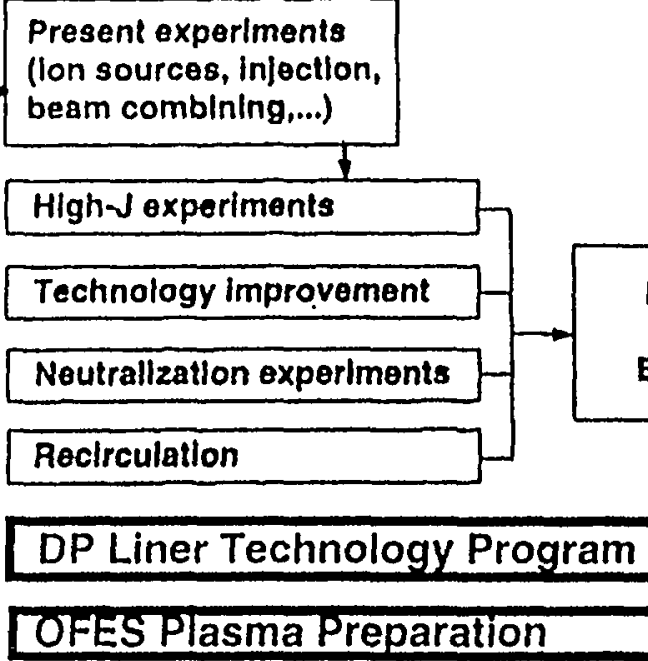

- The feasibility of power production based upon heavy ion drive and magnetized targets should be evaluated.

- Unfortunately, the cost of such a study usually exceeds MTF's projected "proof-of-principle" costs (e.g., HIDIF > 70 man-years). 


\section{The competition is tough!}

- Natural gas power plant (to be installed in Northern California)

- Very low cost of electricity, COE 3k/kWh!

- HYLIFE, COE $\sim 9 k / \mathrm{kWh}$ (1988)

- Low plant capital costs $\sim 600 \$ / \mathrm{kWh}$ !

- HYLIFE, 3,000\$/kWh (1988)

- System efficiency $\sim 60 \%$

- We can not count on the greenhouse argument

- The carbon dioxide emission from natural gas plants has been reduced!

- Wind generators are also in the running in windy areas

- COE 5 $\not / k W h$ 


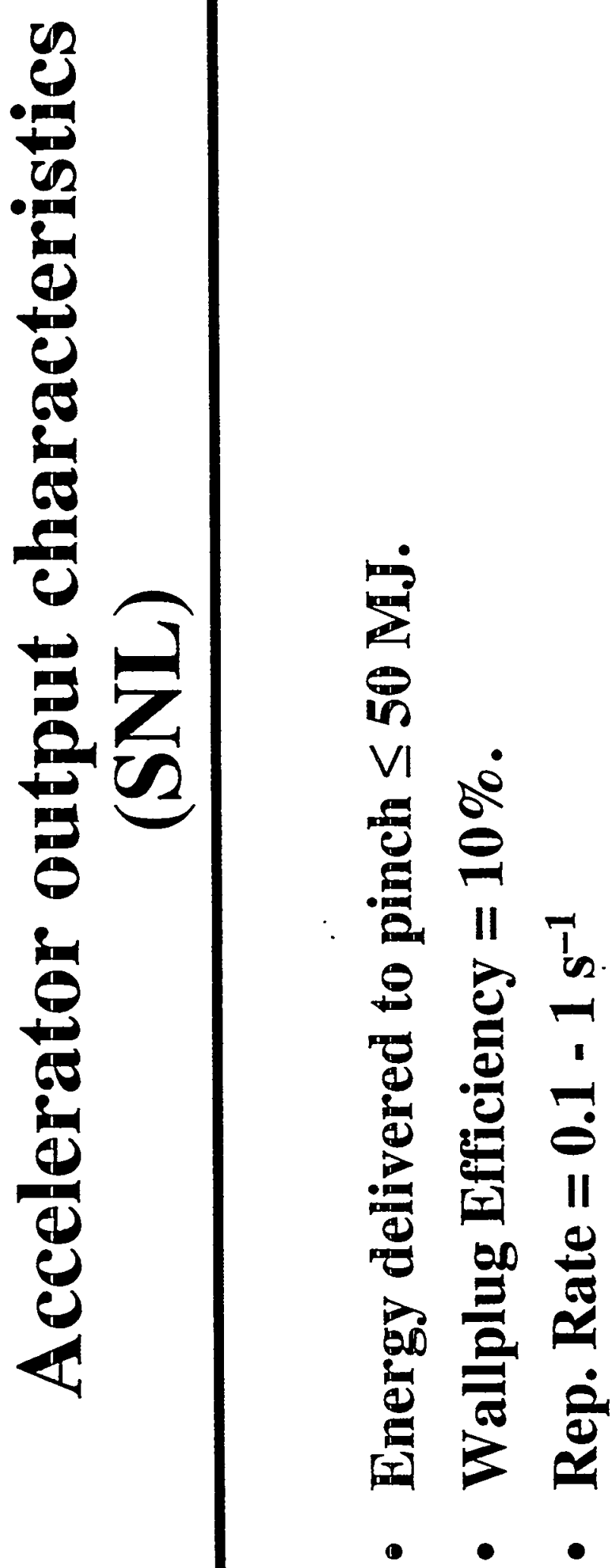




\section{The fusion gain must be high}

- The fusion gain must satisfy:

$\mathbf{f} \eta_{\mathrm{E}} \mathbf{G}_{\text {fusion }} \eta_{\mathbf{x}-\mathrm{ray}} \eta_{\mathrm{A}}=\mathbf{1}$

$\eta_{\mathrm{x}-\text { ray }}=0.8$

$\eta_{E}=0.4$, Electrical energy/thermal energy.

$\eta_{\mathrm{A}}=0.1$, Accelerator efficiency, energy to pinch/energy to accelerator.

$f=0.2$, Recirculating power fraction.

$\mathbf{G}_{\text {fusion }}>\mathbf{1 5 0}$

- High gain $(G>150)$ is required. 


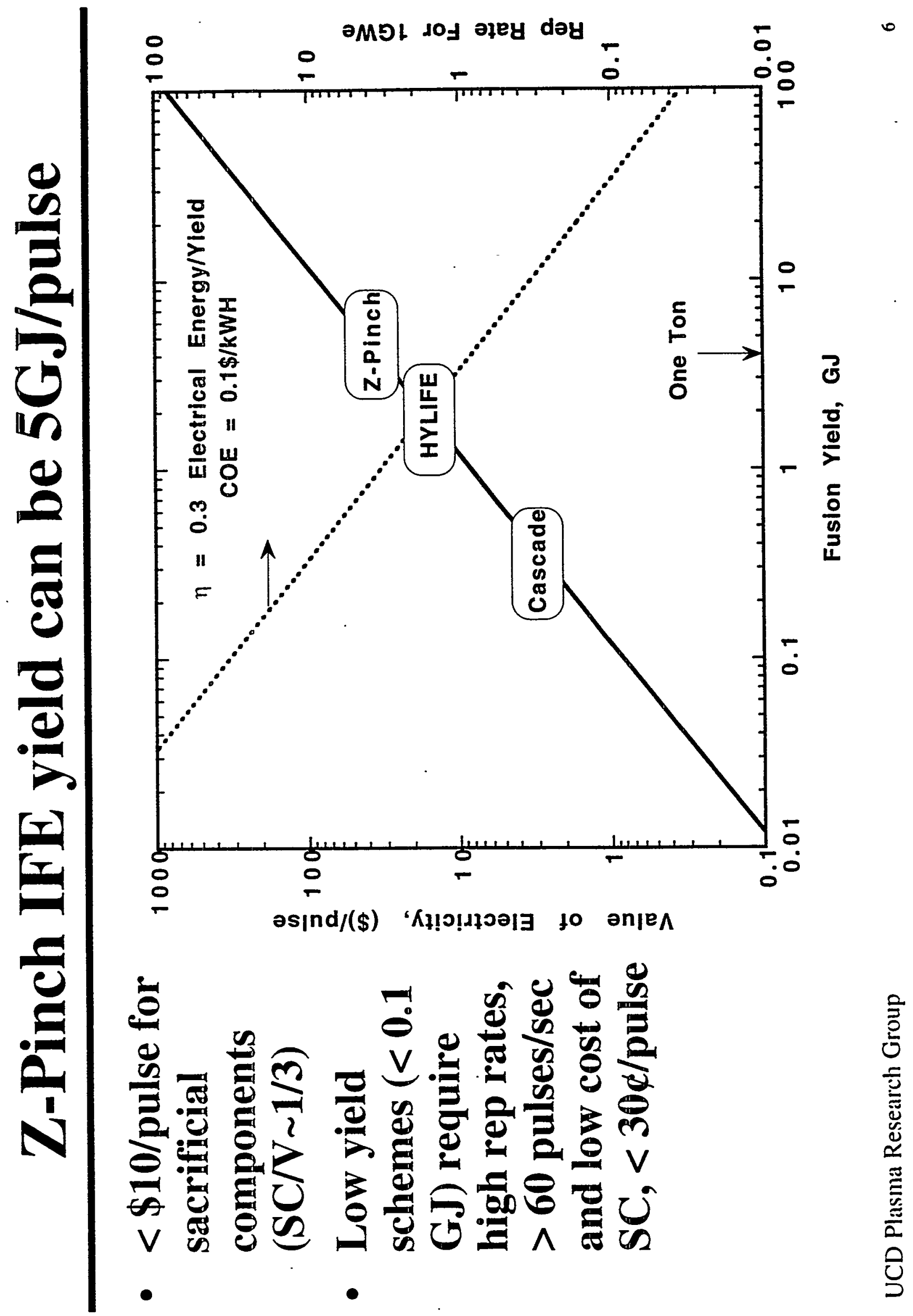




\section{Direct driven schemes}

- Wire array incident on target.

- Quasi-spherical

- DT fiber target

- Wire array incident onto a DT fiber.

- $\ldots$

- ...

- Gain must be high enough! 


\section{X-ray driven schemes}

- Z-pinch driven hohlraum design (J. H. Hammer et.al.) has a gain of 70.

- Accelerator requirements

- 60MA peak current

- 16MJ of $\mathrm{x}$-rays from pinch required

- 1.2 GJ Yield

- Higher gain is required

- Cost of the sacrificial module must be low enough.

- Cost of hohlraum +capsule?

- Cost of electrical connection to load?

- Will wires work?

- Need 1000 wires 


\section{A possible sacrificial module}

- Magnetic field to extend the life of the first wall and to directly convert some of the energy.

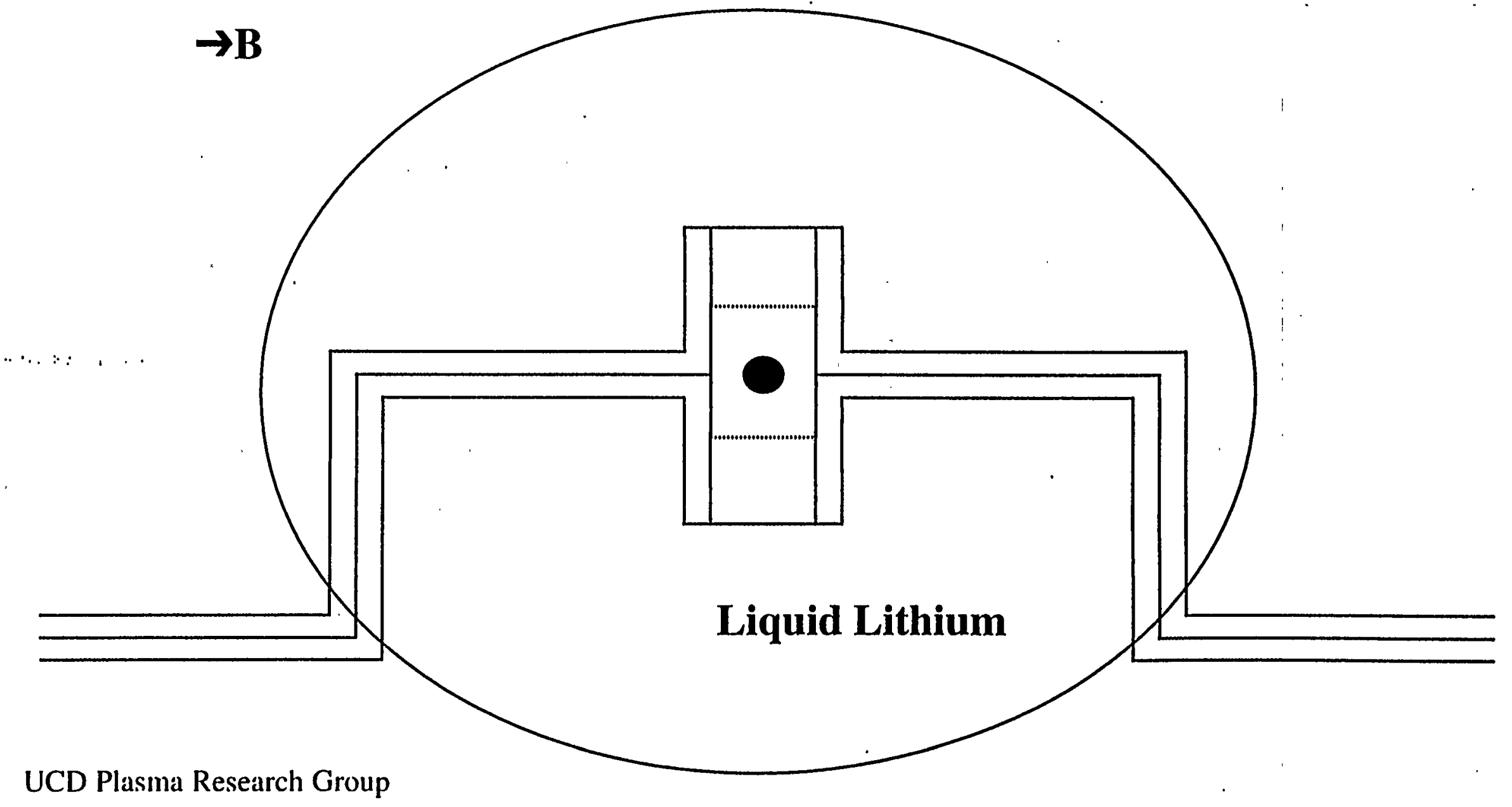




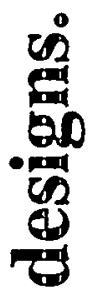

\section{(a)}

a

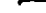

8

2

60

A

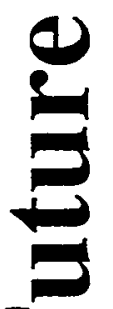

Trad

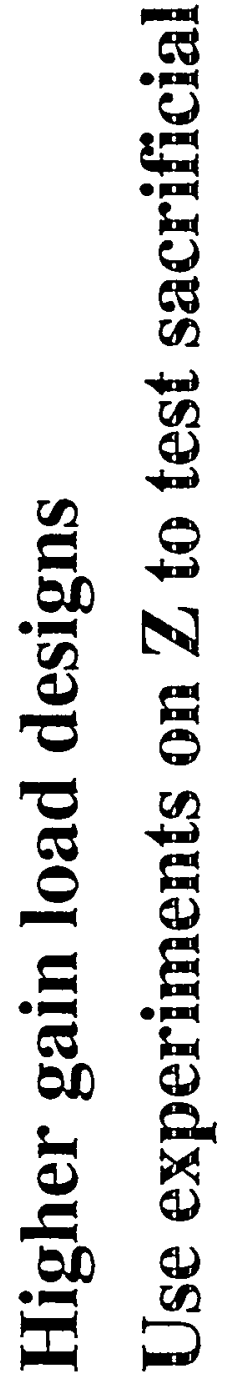




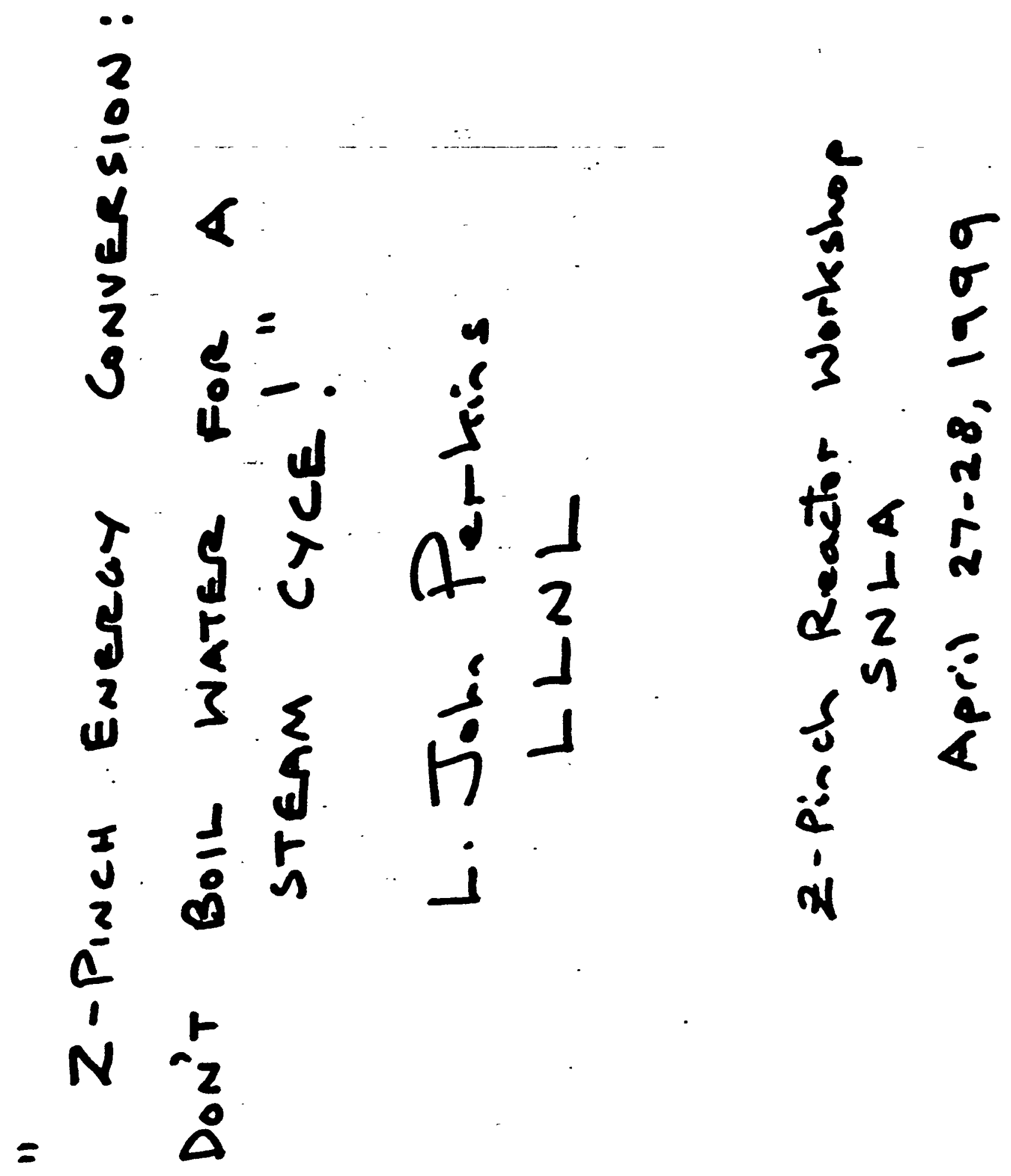




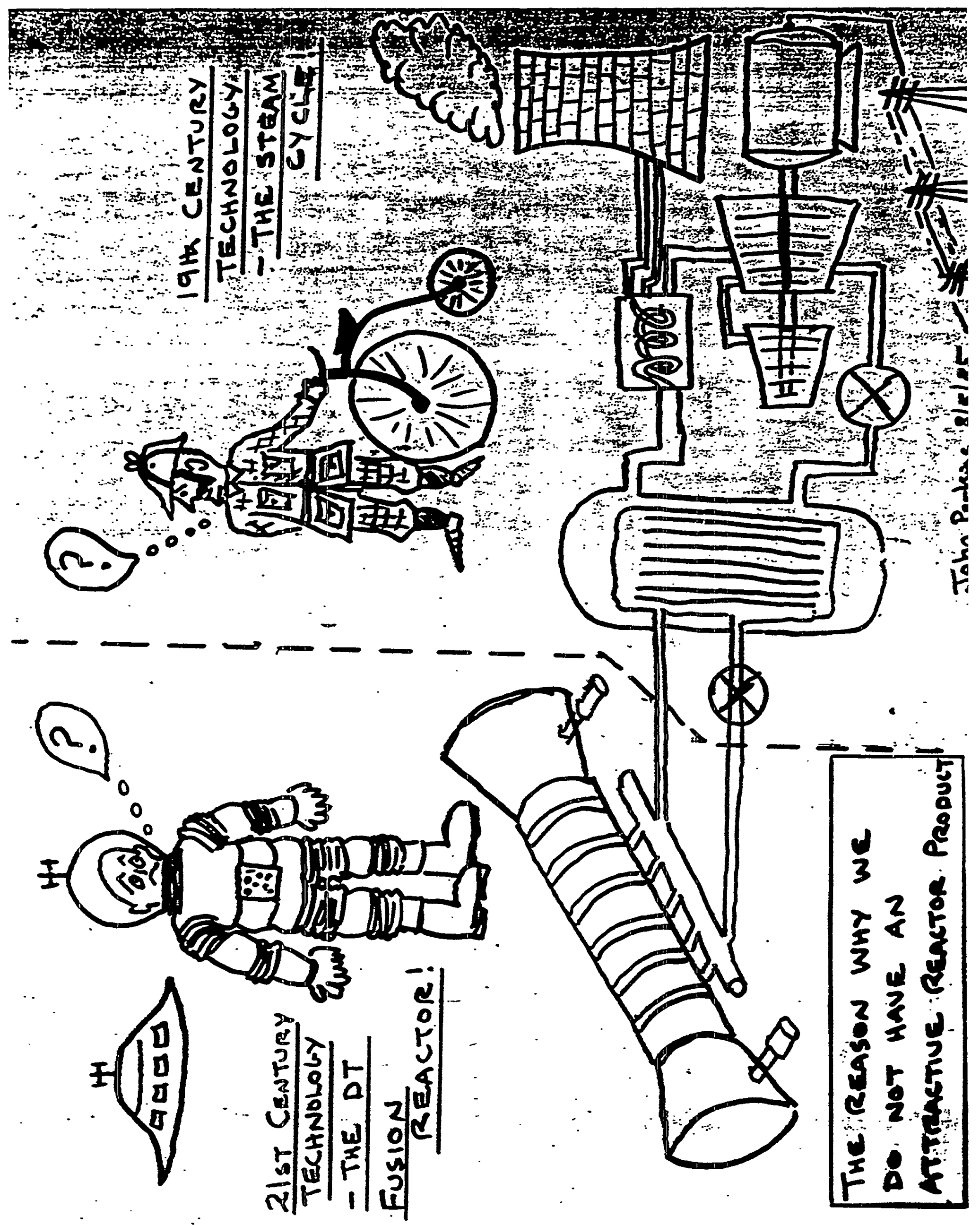




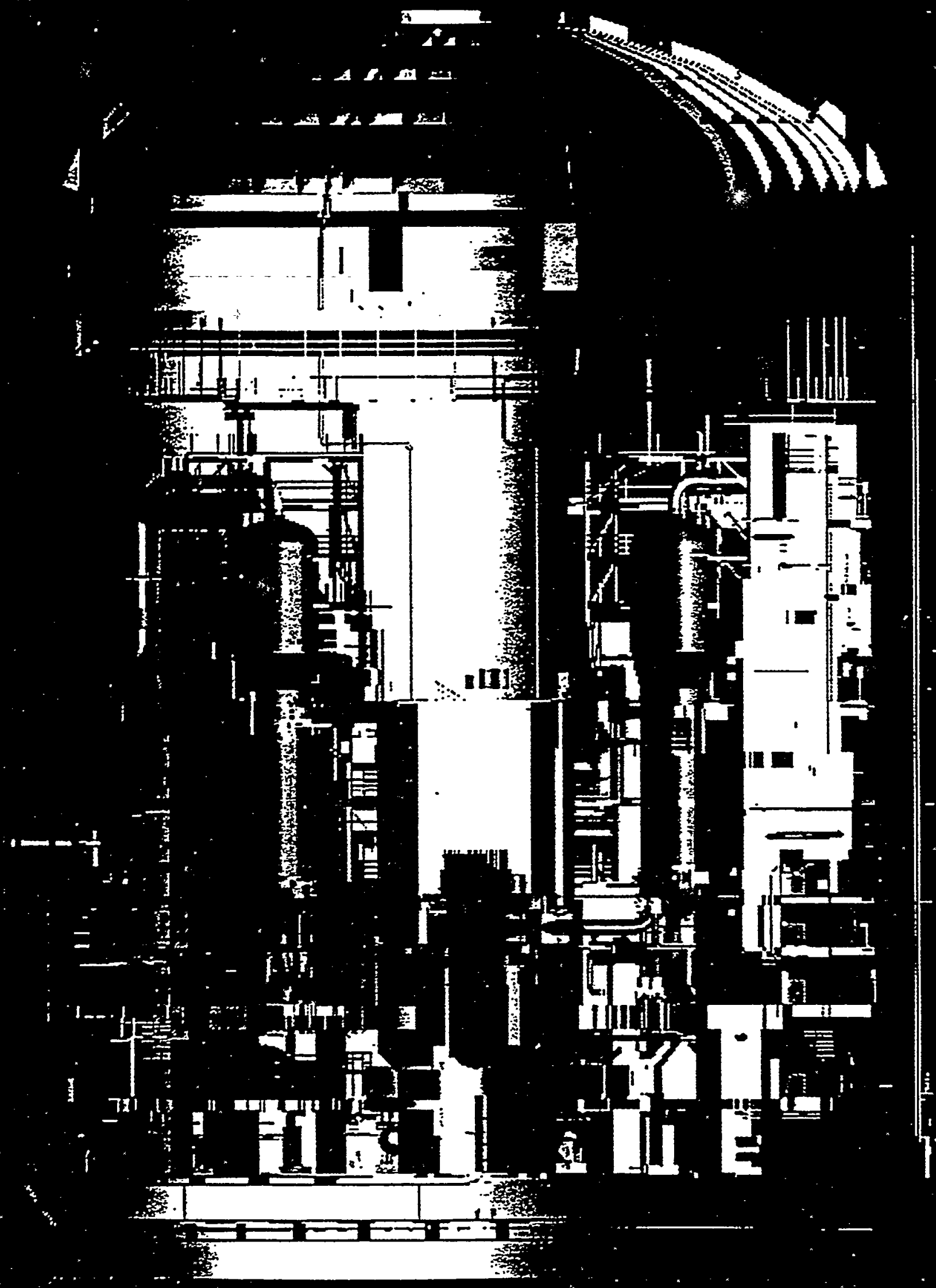

Advanced Fission - The Ultimate Competitor for the $21^{\text {st }}$ Century and Beyond 


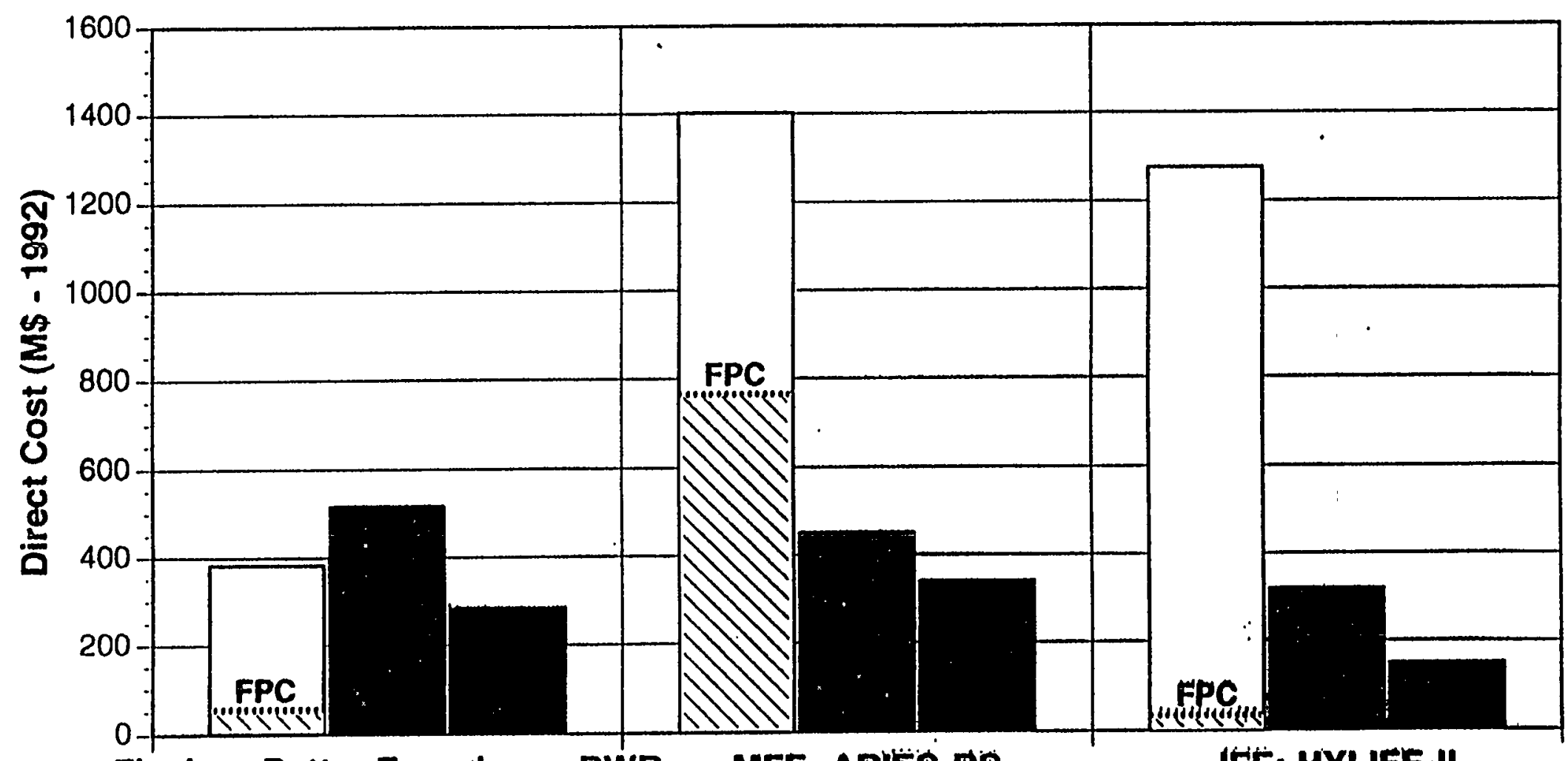

Fission: Better Experience PWR MFE: ARIES-RS . IFE: HYLIFE-II

\section{COMPARISON OF FUSION AND FISSIION CAPITAL COSTS}

(Pertions, Nucl. Trist. Meth, 

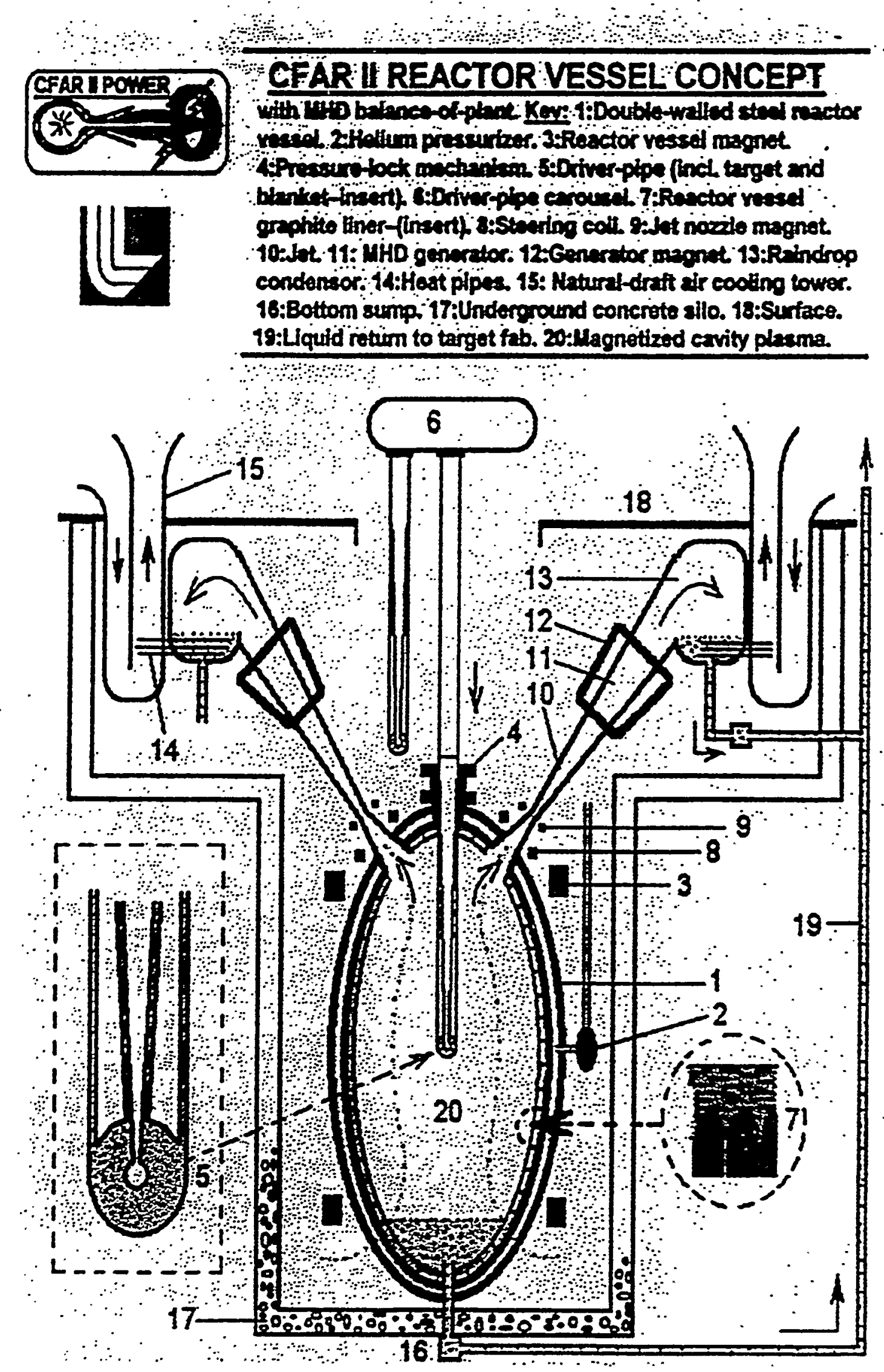

$\log _{a n}(1992)$ 


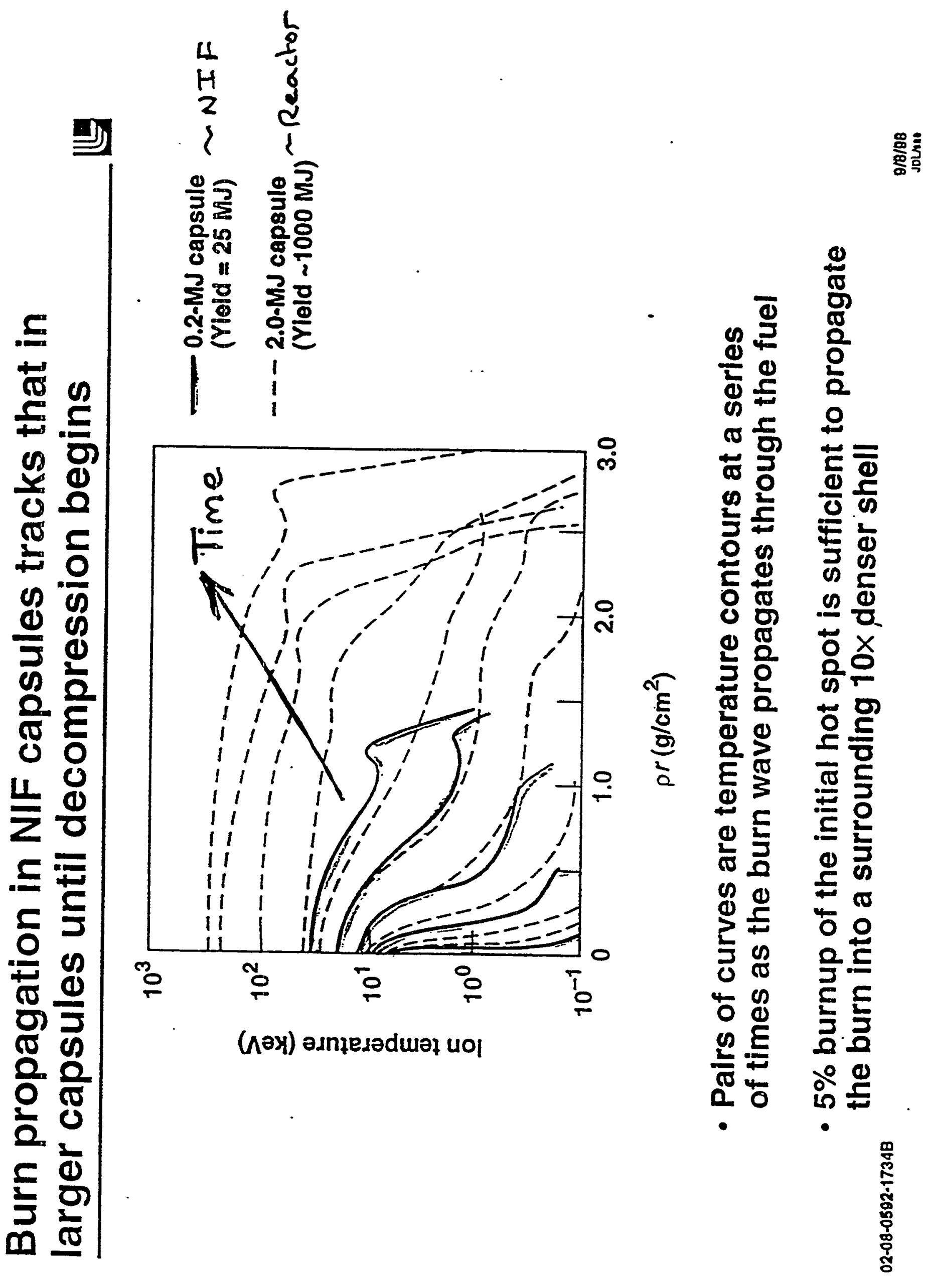




\section{DESCRIPTION OF THE REACTOR COMCEPT}

\subsection{The Energy Cycle}

An inertial-confinement fusion target is. located at the center of a large mass of liquid 1ithium, or "compact blanket" (see Fig. 2.1), of sufficient dimensions for neutron energy absorption and tritium breeding. With this configuration, the outward-directed kinetic energy

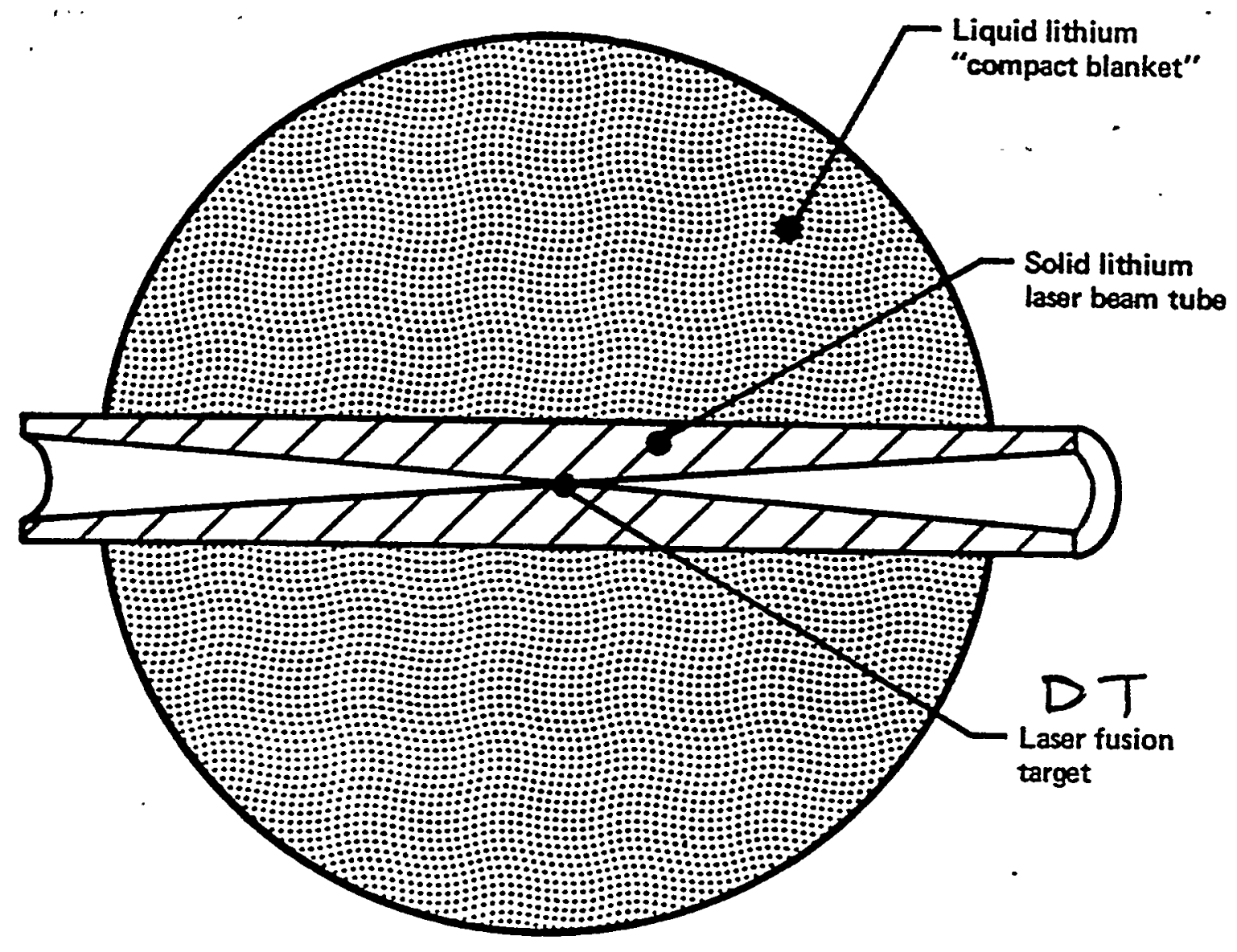

Figure 2.1. The configuration of the 1ithium blanket in a sphere contiguousiy surrounding the ICF fusion plasma offers the maximum possible attenuation of neutrons and the maximum possible shock-induced kinetic energy. 


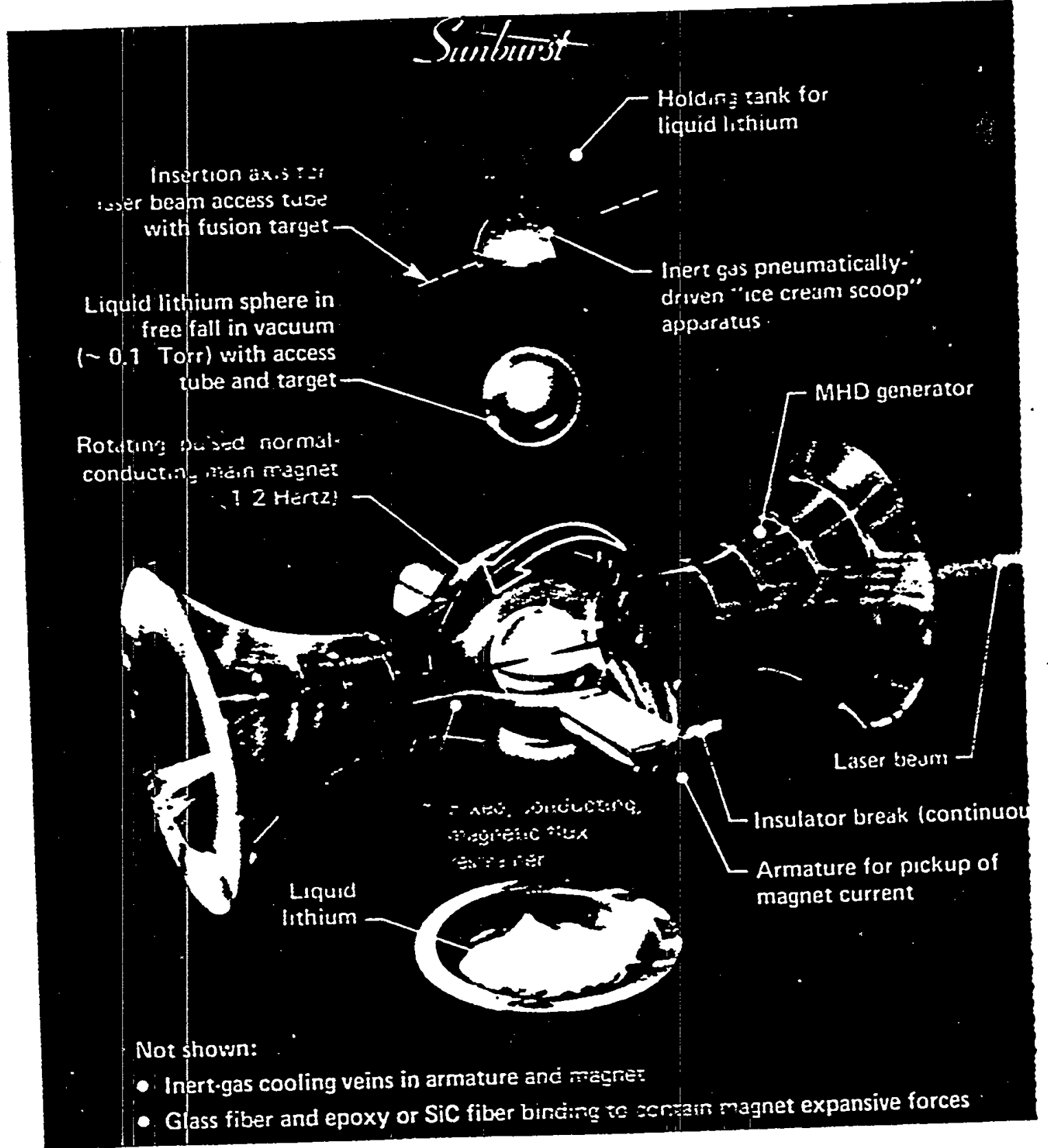

Figure 2.4. The compact blanket concept invites a wide range of imatnative configurations. In this conceptualization, each compac: blanket is captured by a rotating monolithic conducting chamber.

Just prior to the formation and release of the sphere, a tube mat of solid. 1ithium, with a fusion target at its center, is inserted aloi 


\section{Fast Ignition - v- Conventional Hot-Spot Ignition: Effect of Fast Ignitor Spot Diameter.}

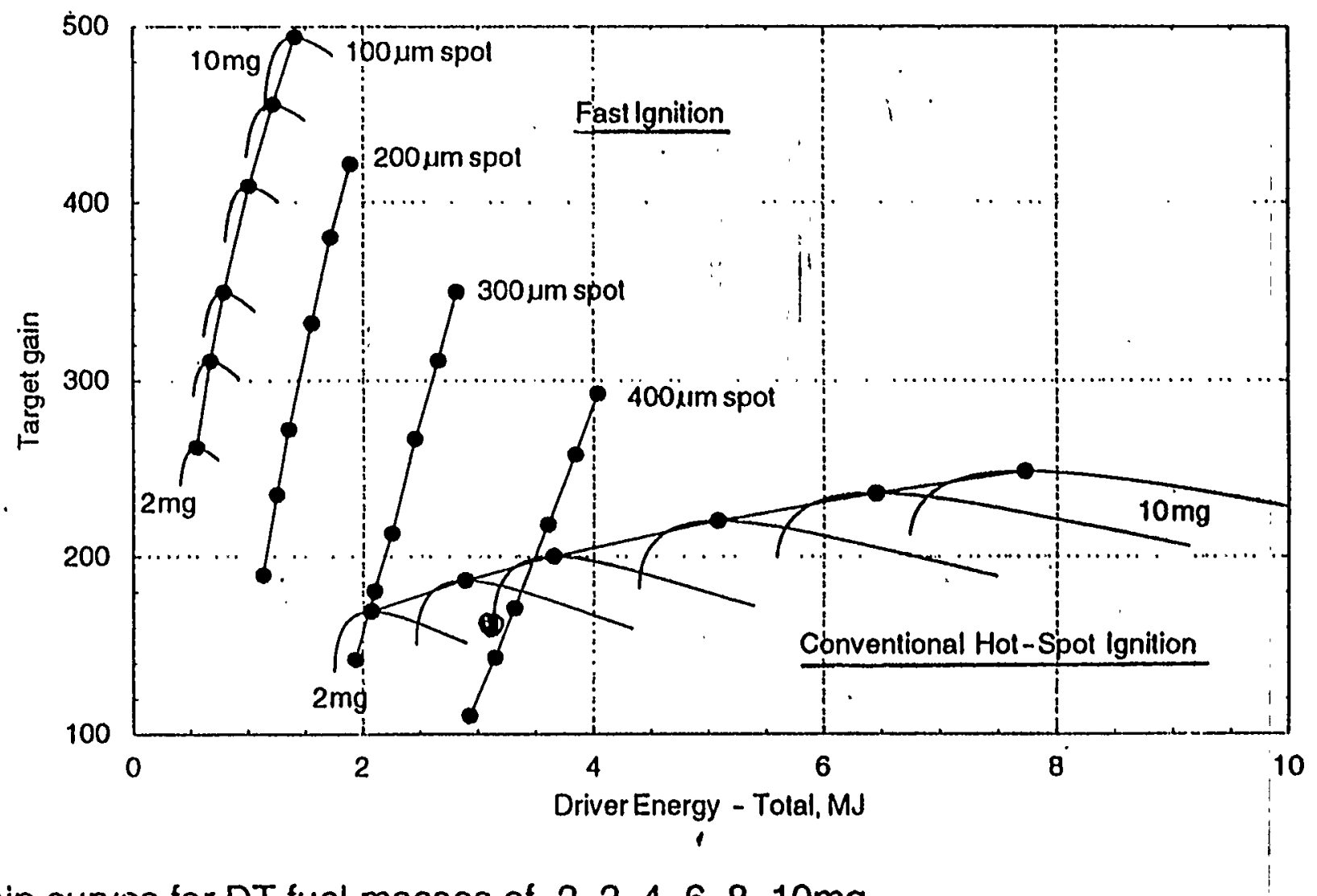

- Gain curves for DT fuel masses of $2,3,4,6,8,10 \mathrm{mg}$.

- Lines connect the locus of maximum gain points for each fuel mass

- Four FI gain curves shown for FI beam spots (FWHM) of 100-400 $\mu \mathrm{m}$ 

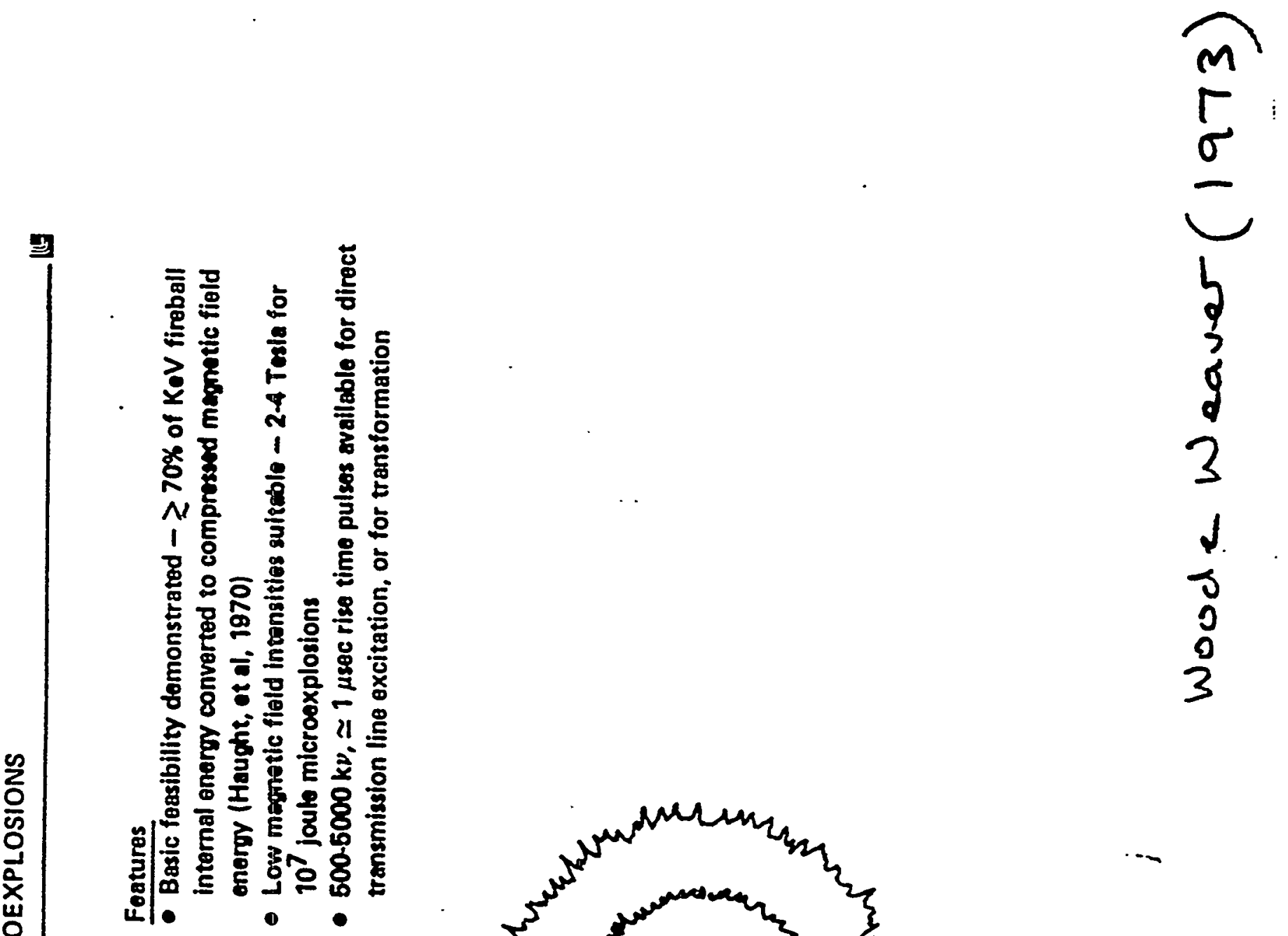

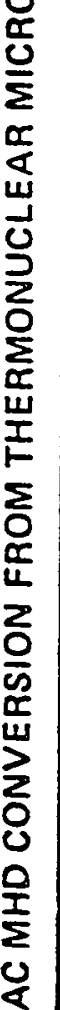

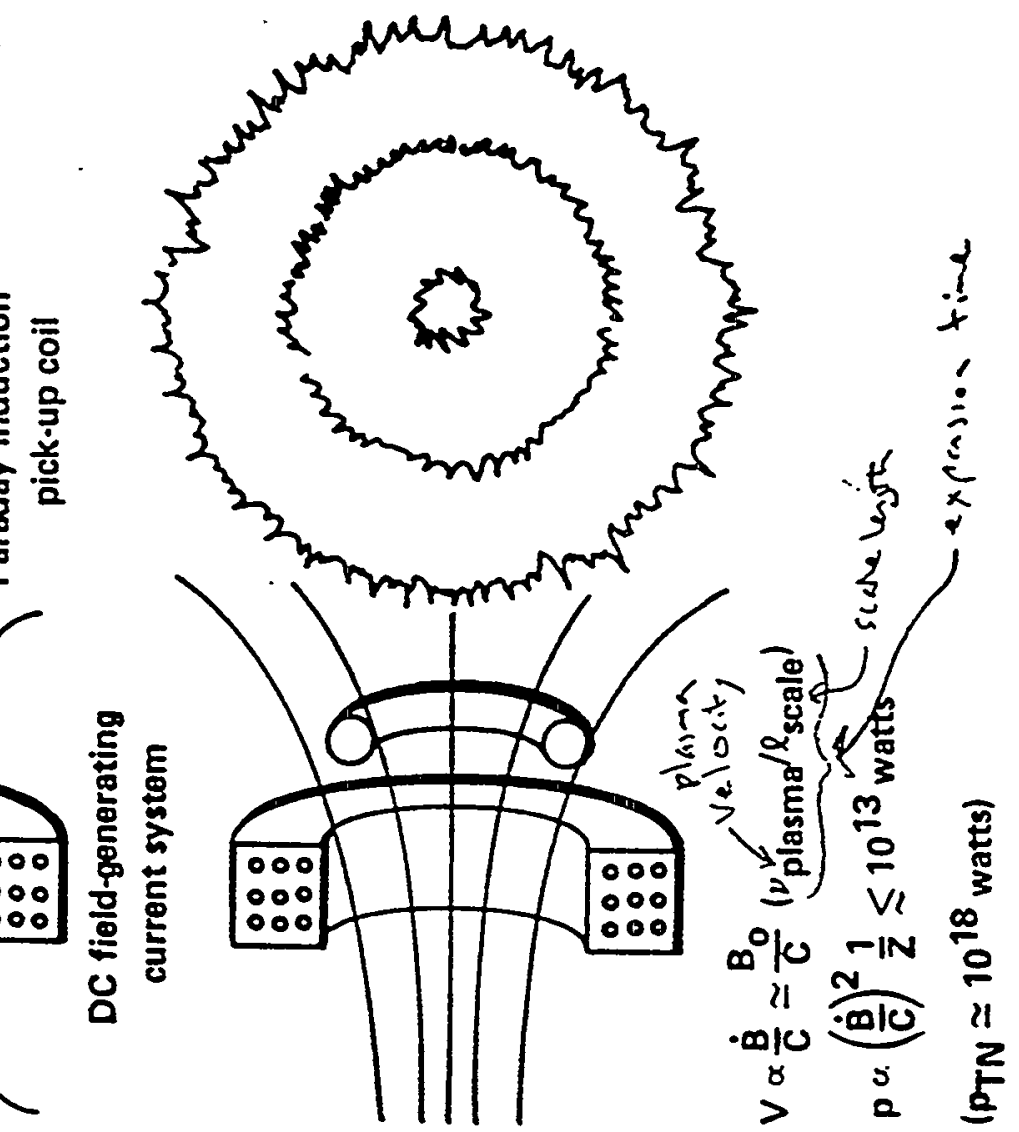



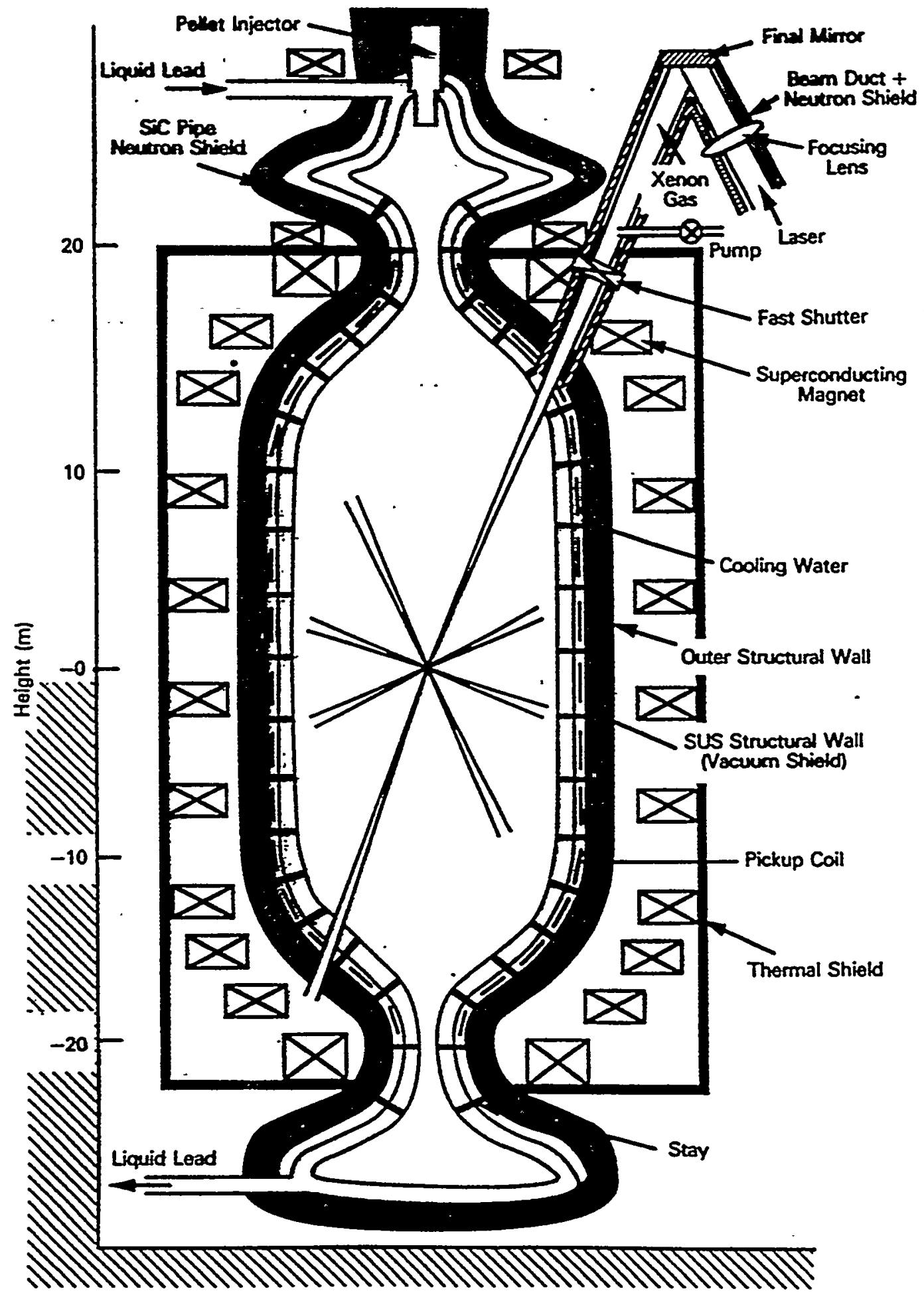

Fig. 1. Cut view of a $D^{3} \mathrm{He}$ ICF reactor with direct energy conversion:

4. Because of the vast differences in the characteristic times, the behavior of thelead vapor generated by bremsstrahlung is not considered.in the process of inductive direct energy recovery.

$$
\text { Mima (1992) }
$$

In the following, the model and analytical results for evaluation of the efficiency of the energy recovery system are presented, where the analysis is based on schemes 1 and 2 
Mima e a. .DIRECT ENERGY CONVERSION FOR D-3He ICF

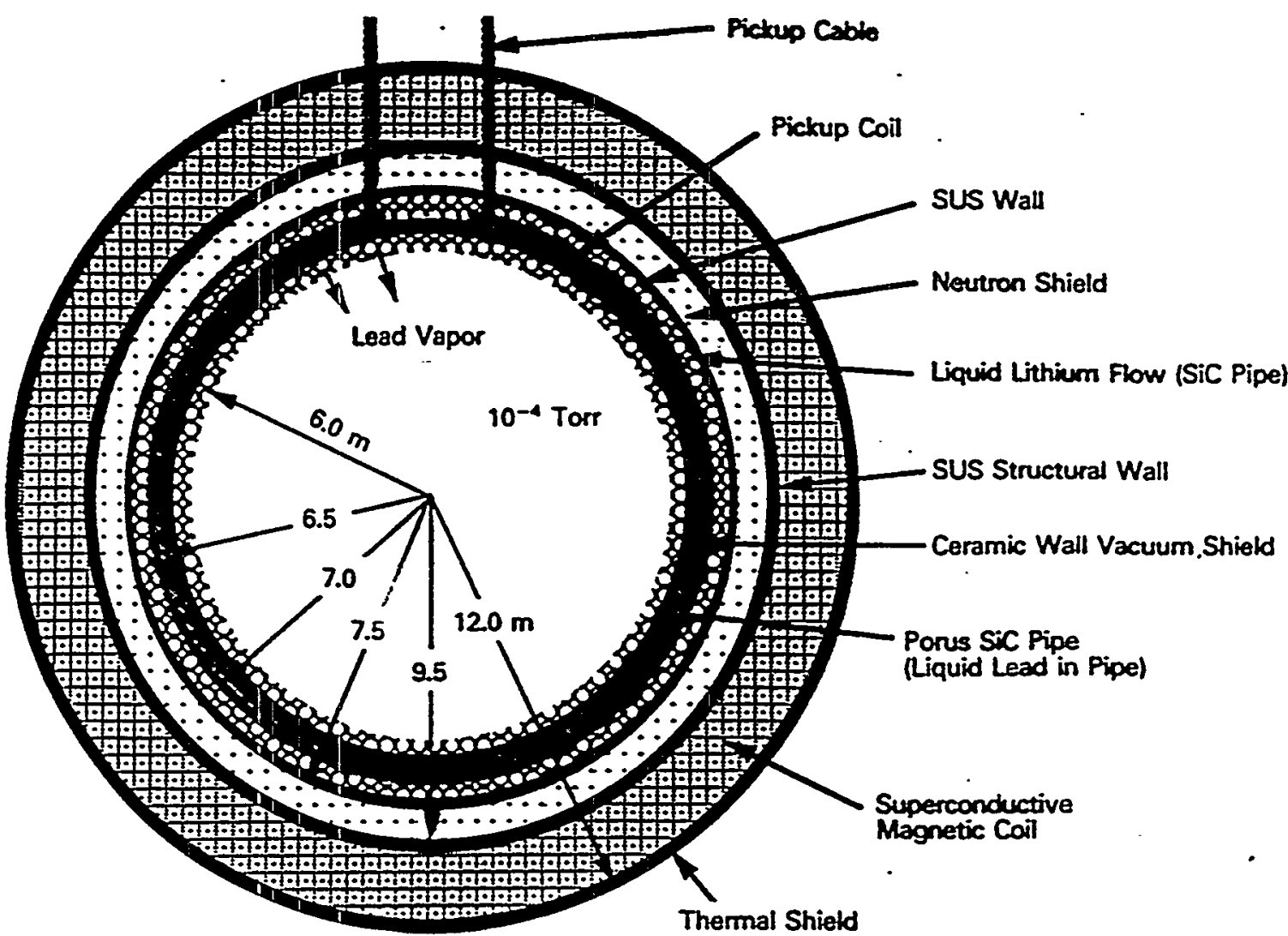

Fig. 2. Central cross section of a $D-{ }^{3} \mathrm{He} I C F$ reactor where a conventional one-turn pickup coil is schematically shown.

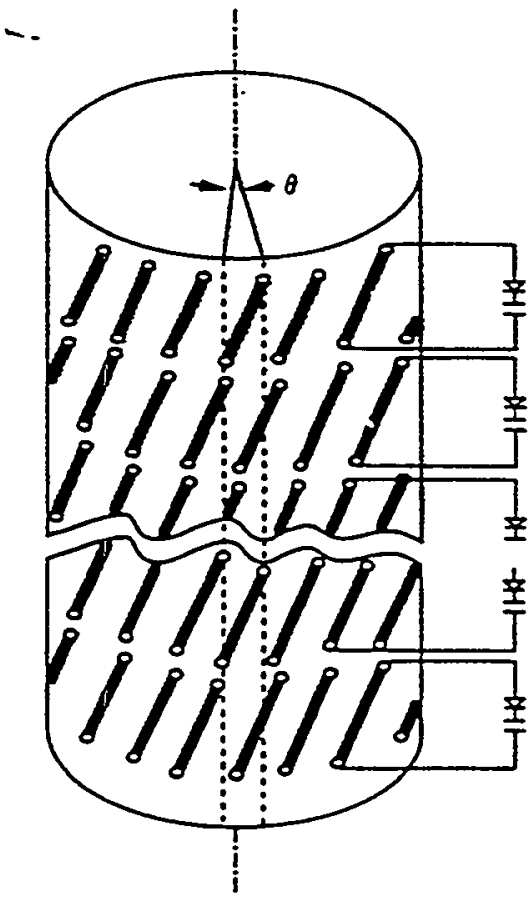

(a)

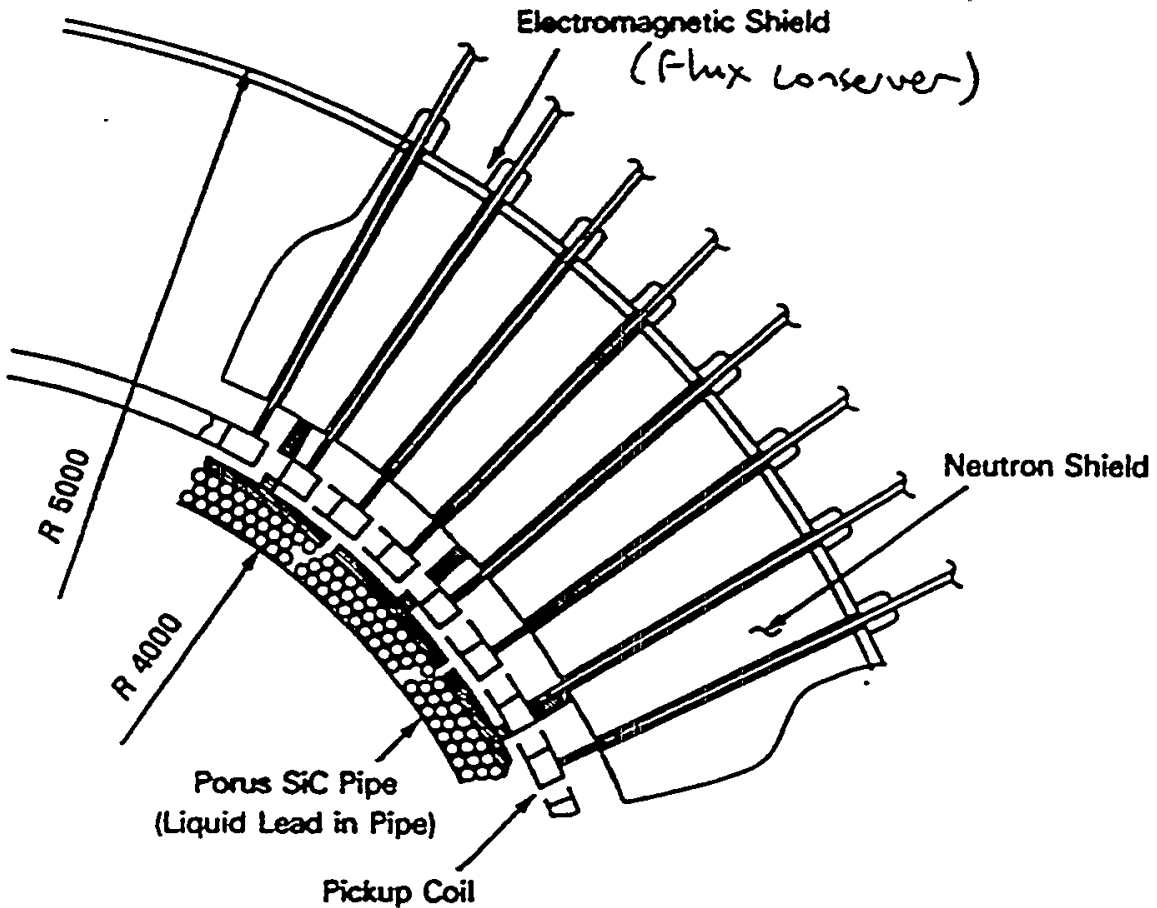

(b)

Fig. 3. (a) Location of pickup segments for alleviation of very high one-turn voltage and (b) cross-sectional view of the SiC pipes and pickup segments. 


\section{Whe Shoúld not be Scared of Large IFE Energy Reservations for the mid 21st Century}

\begin{tabular}{|l|l|l|}
\hline \multicolumn{2}{|c|}{ Hydroelectric } \\
\hline \multicolumn{1}{|c|}{ Country } & \multicolumn{1}{c|}{ Capacity $\left(\mathrm{GW}_{\mathrm{e}}\right)$} \\
\hline (Three Gorges) & (China) & $(18.2)$ \\
\hline Guri & Venezuela & 10.3 \\
\hline Itaipu & Brazil/Paraguay & 7.4 \\
\hline Grand Coulee & United States & 6.8 \\
\hline Sayano Shushensk & Russia & 6.4 \\
\hline Krasnoyarsk & Russia & 6.0 \\
\hline La Grande 2 & Canada & 5.3 \\
\hline
\end{tabular}

\begin{tabular}{|l|l|l|}
\hline \multicolumn{2}{|c|}{ Nuclear Fission } \\
\hline \multicolumn{1}{|c|}{ Plant } & \multicolumn{1}{|c|}{ Country } & \multicolumn{1}{c|}{ Capacity $\left(G W_{\mathrm{e}}\right)$} \\
\hline Bruce & Canada & 6.5 \\
\hline Gravelines & France & 5.5 \\
\hline Paluel & France & 5.3 \\
\hline Cattenhom & France & 5.2 \\
\hline Zaporozhye & Ukraine & 4.8 \\
\hline Fukishima/Ohkuma & Japan & 4.6 \\
\hline Fukishima/Naraha & Japan & 4.3 \\
\hline L. John Perkins LLNL 4/12197 & \\
\hline
\end{tabular}




\section{Where Should We Invest Effort in Advanced IFE Concepts?}

Advanced high-gain targets: Closed-coupled houlrahms, indirect-drive, onesided, fast ignition, ....

- Very high-gradient accelerators: E.g., dielectric wall accelerators for heavy-iondriven IFE with accelerating gradients of 10 's $\mathrm{MV} / \mathrm{m}, \ldots$...

- Advanced, liquid vortex blankets: To minimize isochoric heating and bulk liquld break-up and facilitate chamber clearing for DT energy conversion.

- Advanced, non-DT target fuels: DD or $\mathrm{D}^{3} \mathrm{He}$ main fuel with self-breeding DT sparkplugs ( $T$ fraction $\sim 1-2 \%$ ). Fraction of yield in fast neutrons $\leqslant 20 \%$

- Advanced, in-situ energy conversion: MHD plasma conversion, magnetic flux compression, ....

$\Rightarrow$ Don't boil water for a steam-cycle, i.e., avoid coupling our $21^{\text {st }}$-century energy source to a $19^{\text {th }}$-century energy conversion technology! 


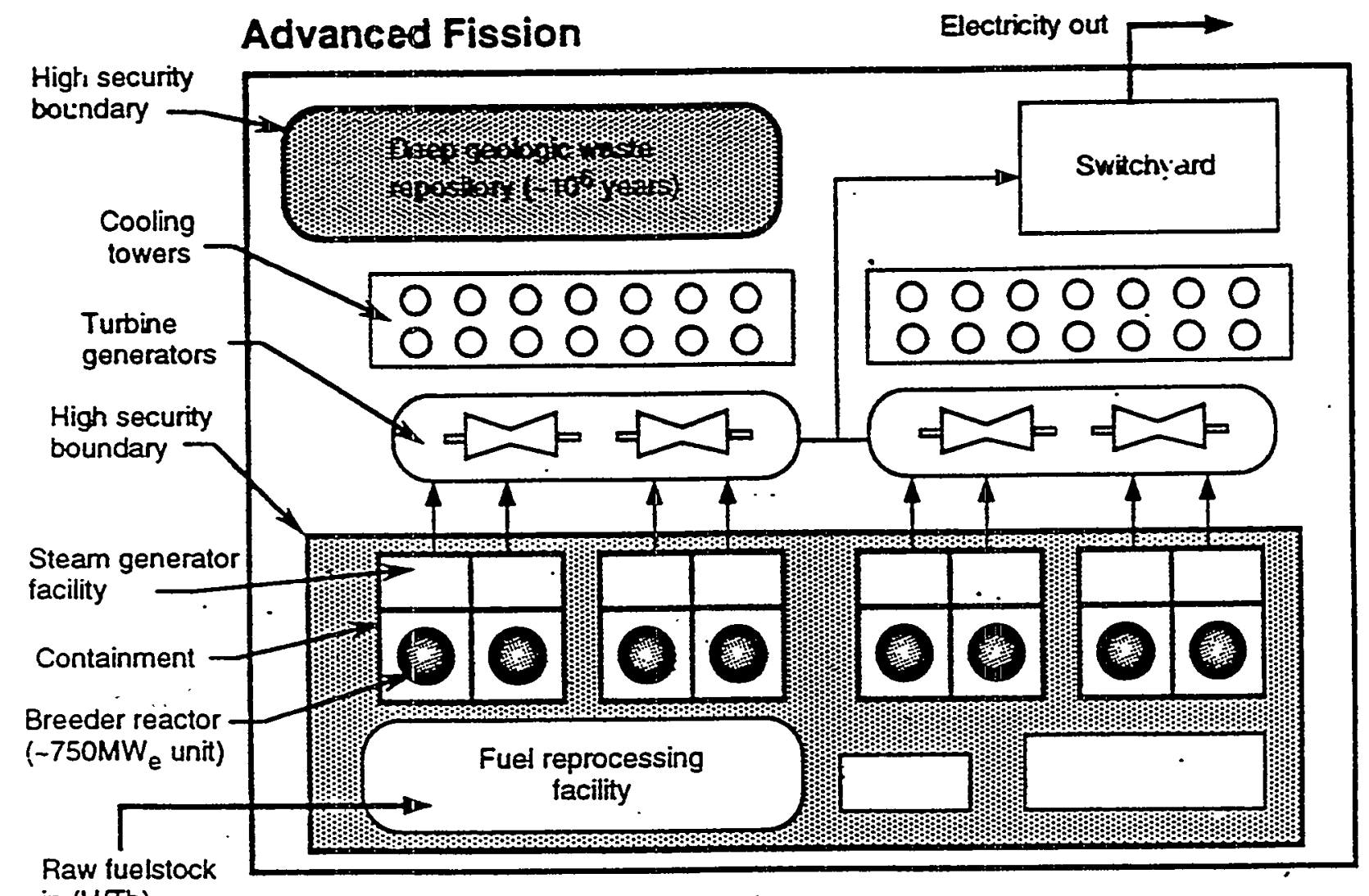

in $(\mathrm{U} / \mathrm{Th})$

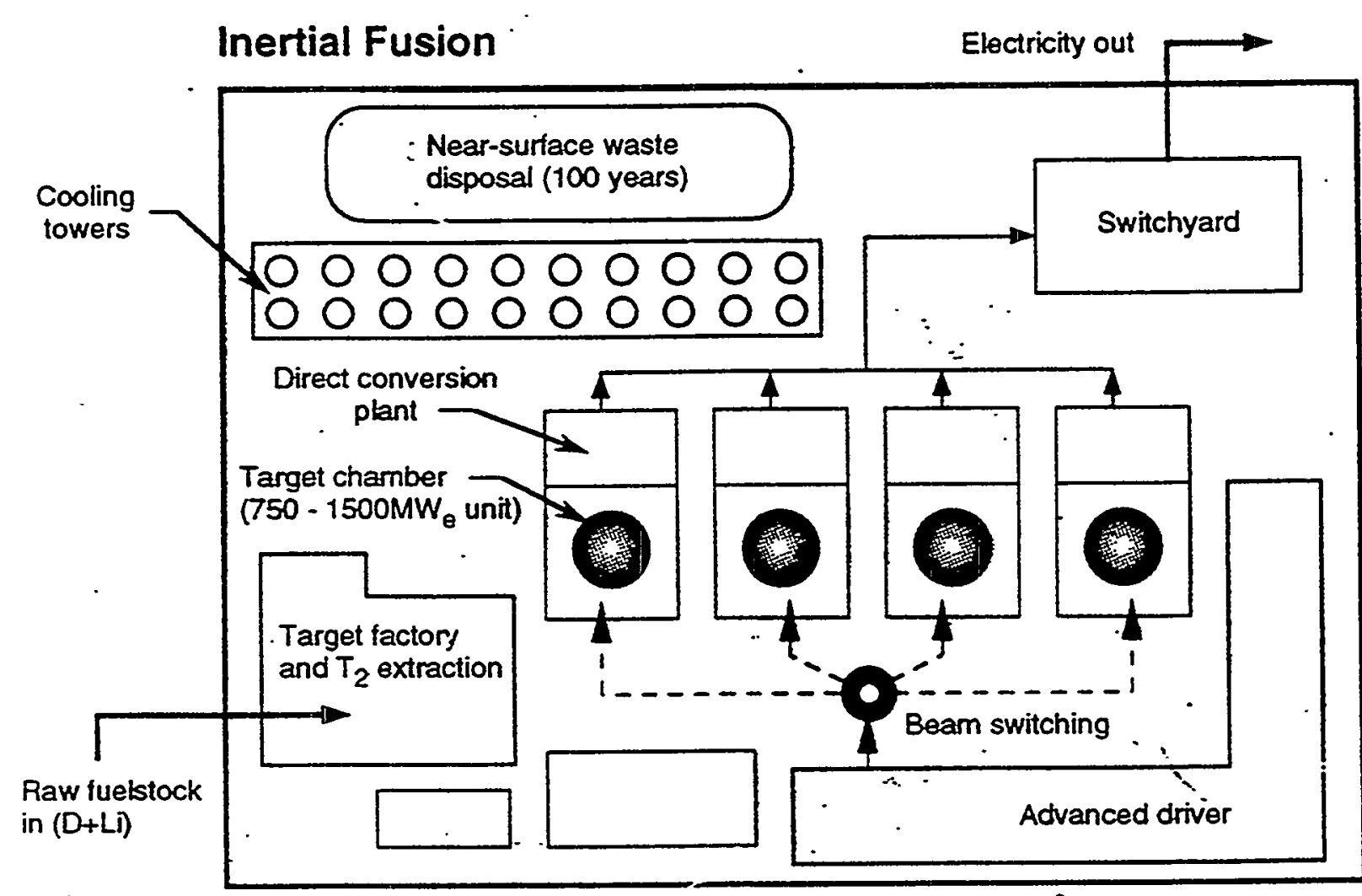




$$
\begin{aligned}
& \text { MT F } \\
& \text { Matching } \\
& \text { The TARget } \\
& \text { TO the DrIVER }
\end{aligned}
$$

RON KIRKPATRICK

Los Alamos National Lab.

at z-Pinch Work shop Saudia National Lab April 27-28, 1949 
WHAT CHARACTERIZES DRIVER?

POWER

PULSE LENGTH

FOCUS

OR: Energy, Power, Intensity

- ONTO TARGET

$O R$ :

TEMPERATURE

+ dURATION 
WHAT CHARACTERIZES

THE TARGET?

MASS

SIZE

MATERIALS scale lengths

MODE OF IGNITION:

(minimum Implosion VELOCITY)

$E \sim \epsilon M$

$\rho+r$ set by mode of IGNITION

$M+e \rightarrow R$

$\tau \simeq R / v$

$P \sim E / \tau$

$F \sim P / R^{9-1} \quad\left(F \sim G T^{4}\right)$ 


$$
\begin{aligned}
P & \sim E r / R \\
F & \sim E\left(\frac{V}{R}\right) / R^{g-1} \quad g=1,2,3 \\
& \sim E r / R^{9} \\
& \sim E M r / R^{9} \\
\sigma T^{4} & \sim E r \rho
\end{aligned}
$$

MODES of IGNITION

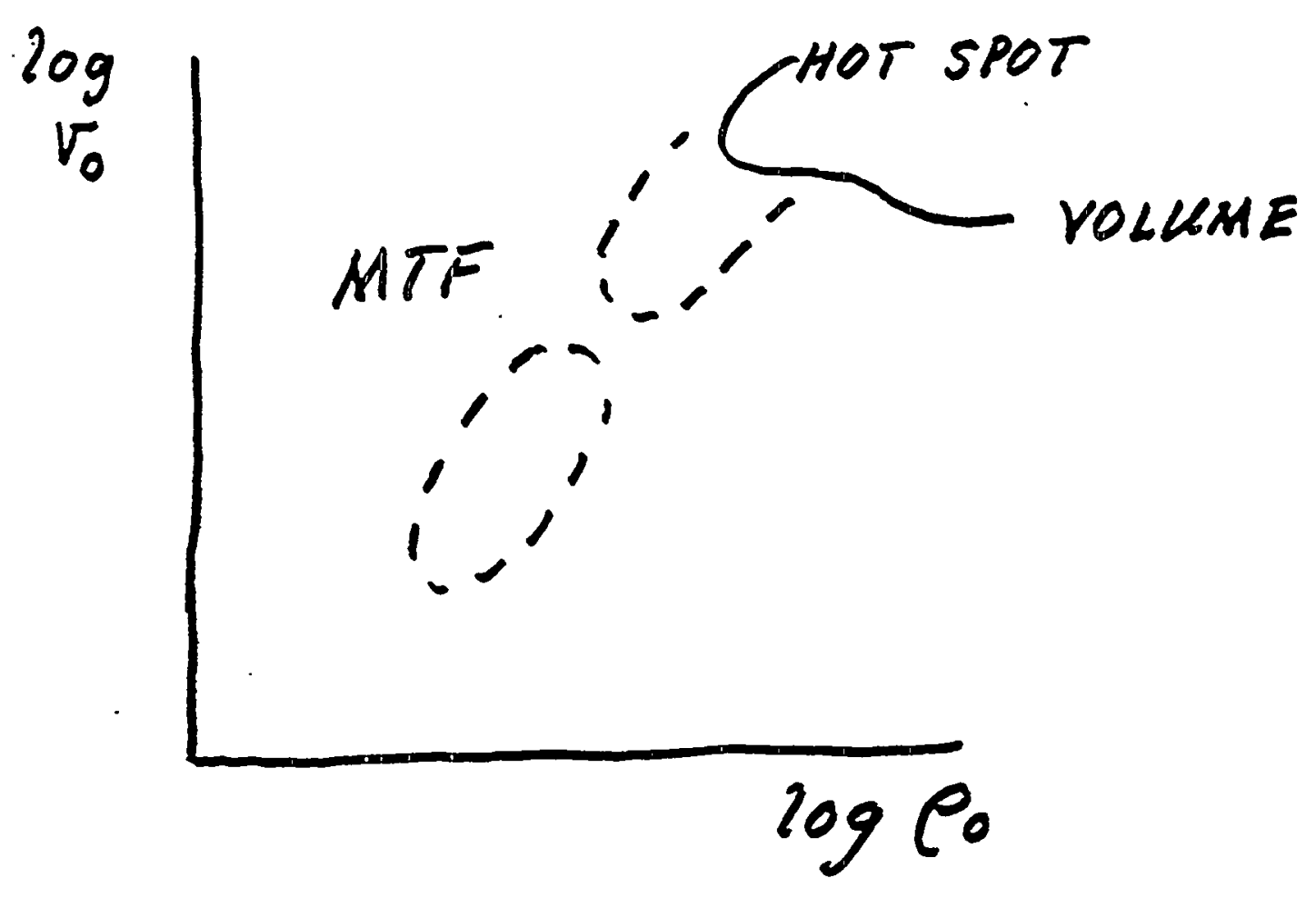


FINDING A MATCH
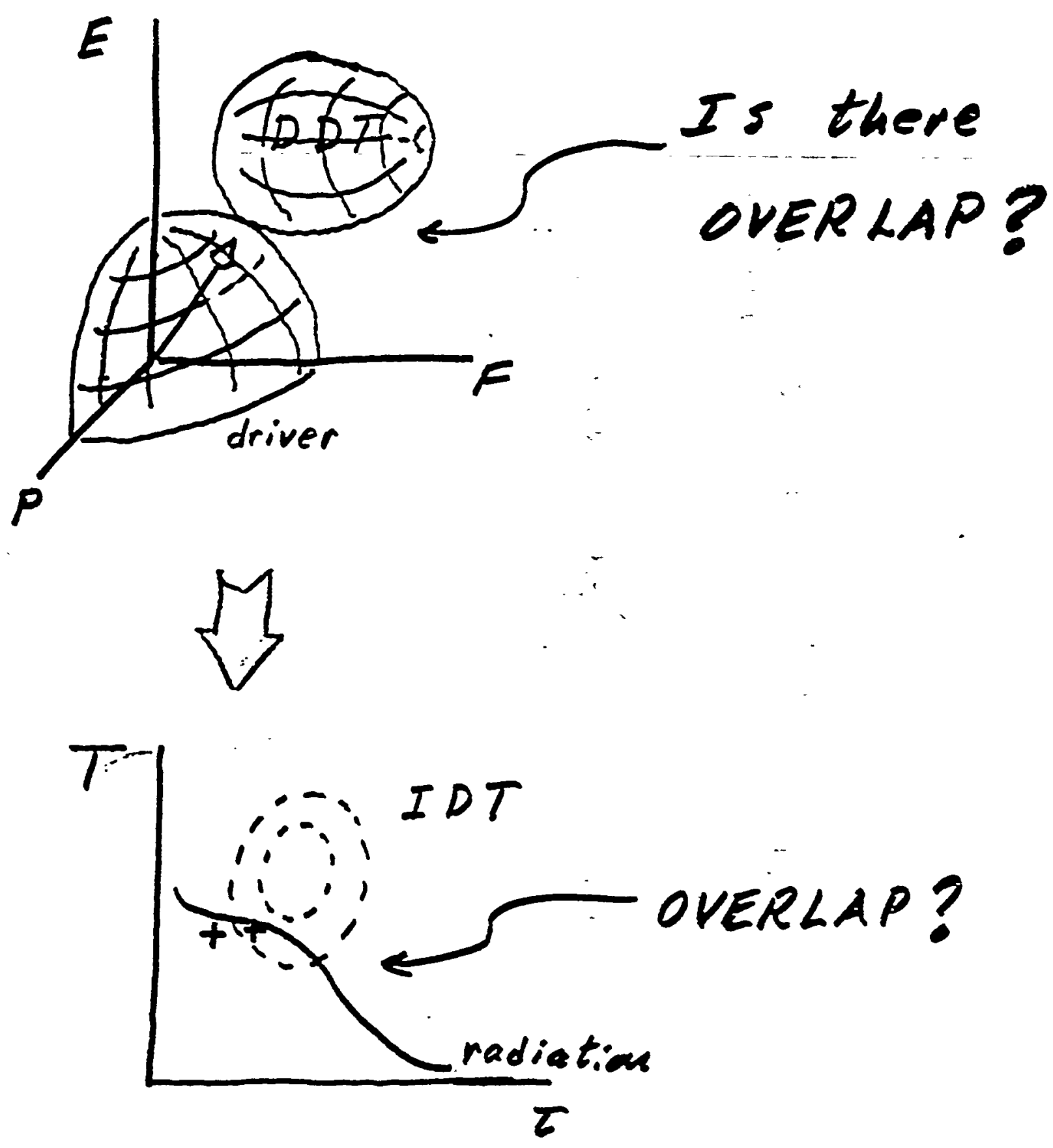
MAGNETIZED TARGETS FOR FAST Z-PINCH IMPLOSIONS

D. Ryutov

SNL, Albuquerque, 04.27-28,99 
BD IMPLOSIONS:

$$
\begin{aligned}
& n=n_{0} C^{3}, T=T_{0} C^{2}, \\
& B=B_{0} C^{2}, \beta=\beta_{0} C \\
& C=10 \\
& n_{0}=10^{18} \mathrm{~cm}^{-3}, \quad T_{0}=100 \mathrm{eV} \\
& B_{0}=10^{5} G, \quad \beta_{0}=1 \\
& L_{0}=5 \mathrm{~cm}, \quad r_{0}=1 \mathrm{~cm} \quad(F R C)
\end{aligned}
$$

SLOW. IMPLOSIONS, $V_{\text {Liner }} \ll C_{S \text { (plasma) }}$

("FAst" IS a RELATIVE TERM) 


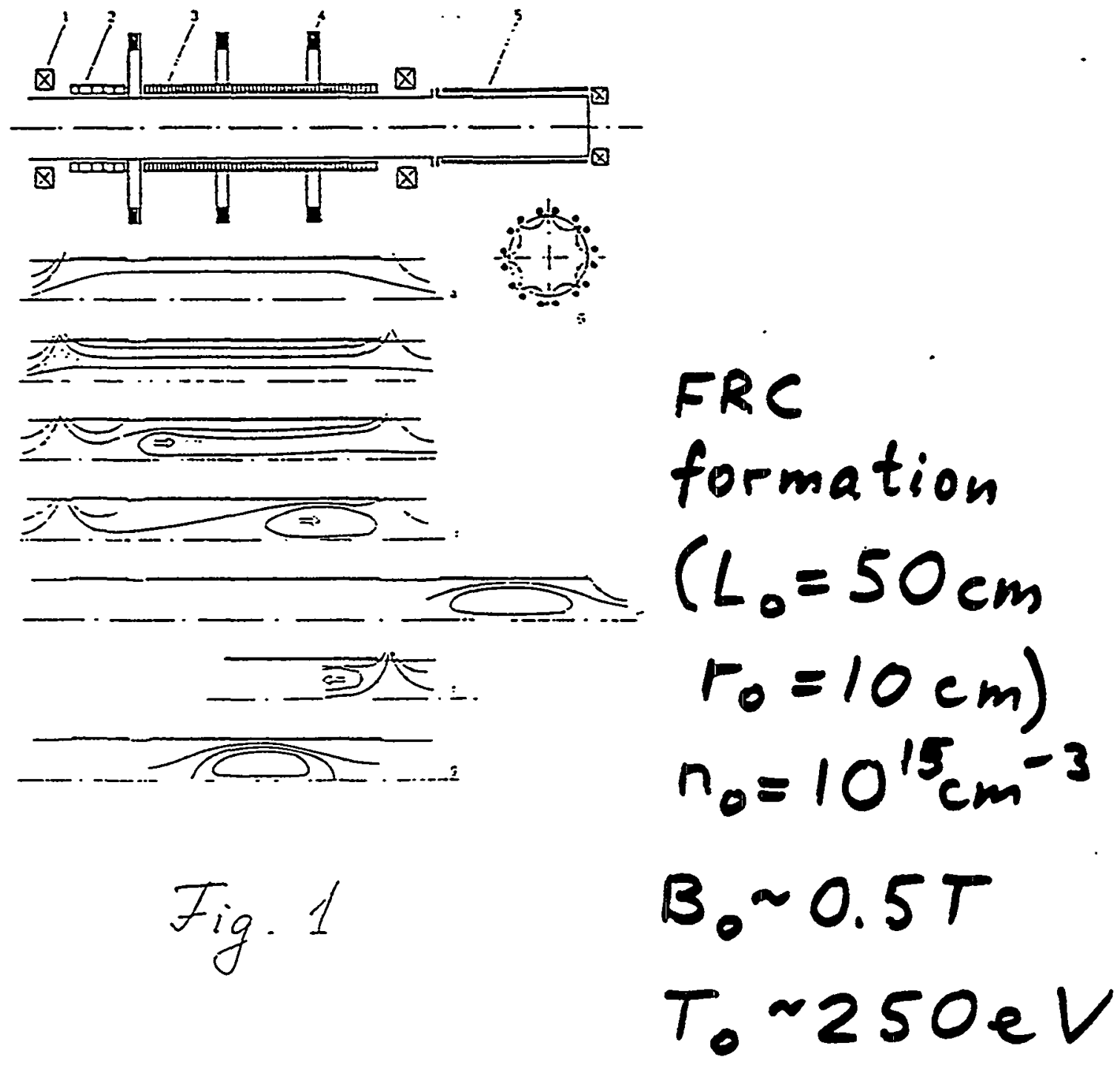




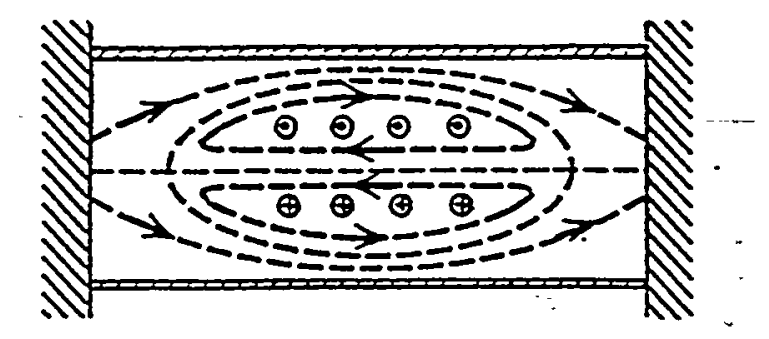

a

$$
\begin{aligned}
& \text { Prolate } \\
& \text { spheromak }
\end{aligned}
$$

b

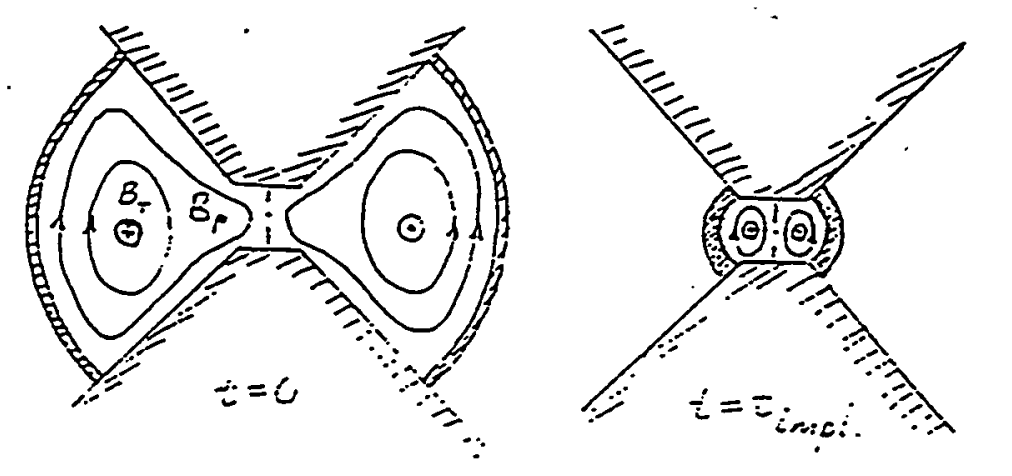

"Spherical" spheromak

$$
\text { I.g. } 2
$$



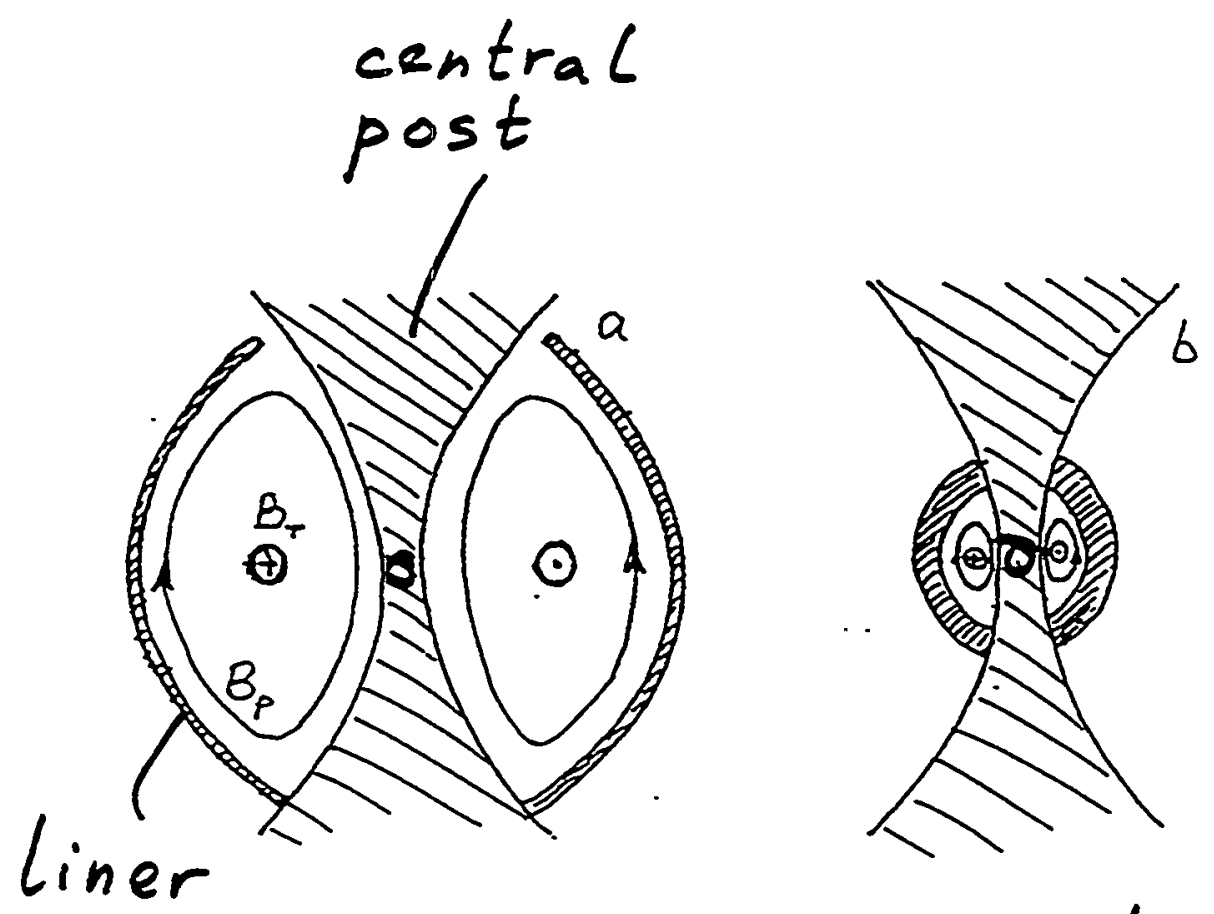

Spherical tokamak

Fig. 3

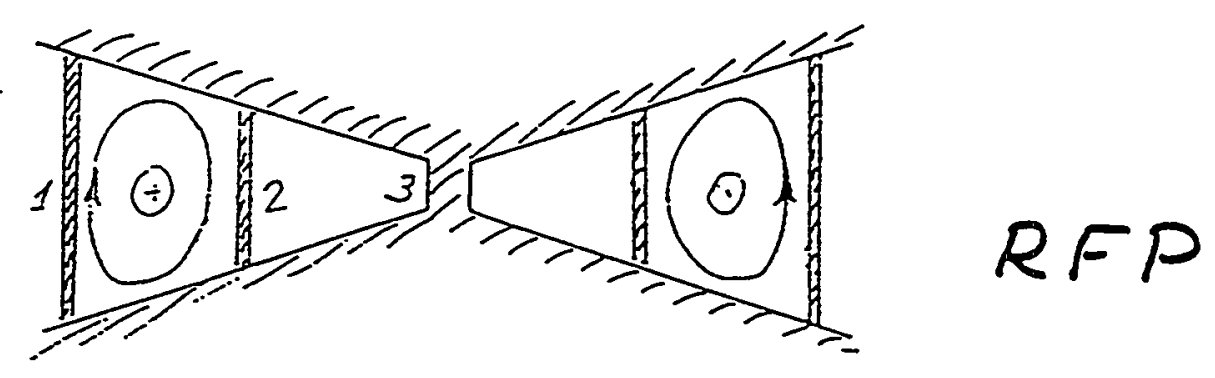

Fig. 4 


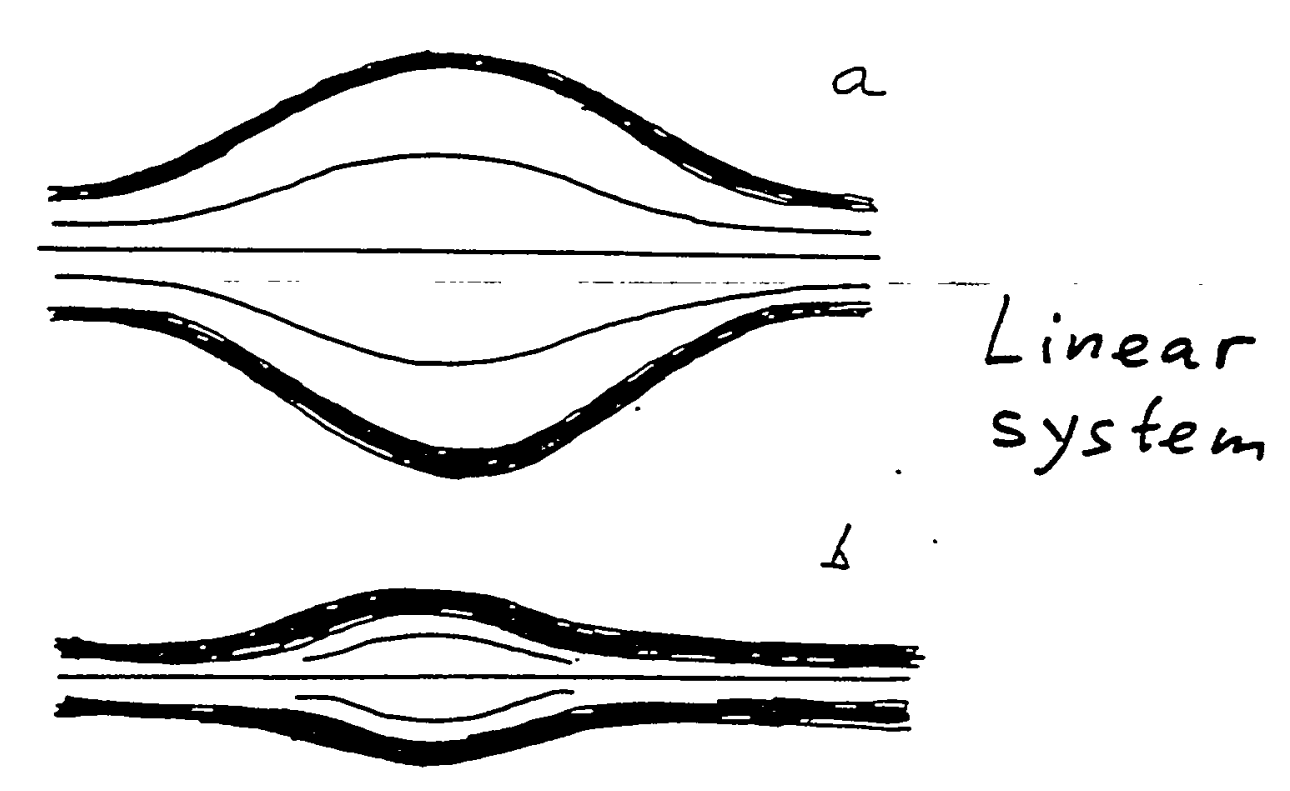

Fig. 5 
TABLE 1. General comparison of viarious candiclate configurations for 3D implosions

\begin{tabular}{|c|c|c|c|c|c|c|}
\hline Configuration & $\begin{array}{l}\text { Plasma bela } \\
\text { demonstrated } \\
\text { cxperimentally }\end{array}$ & $\begin{array}{l}\text { Can the initial } \\
\text { configuration be } \\
\text { crcated inside a } \\
\text { conducting } \\
\text { liner? }\end{array}$ & $\begin{array}{l}\text { Is the confining } \\
\text { inagnetic field } \\
\text { cvery where } \\
\text { langential to the } \\
\text { liner surface }\end{array}$ & Main problem & Main advantage & $\begin{array}{l}\text { Amount of } \\
\text { information } \\
\text { collected from the } \\
\text { studies of related } \\
\text { magnetically. } \\
\text { confined systems } \\
\end{array}$ \\
\hline FRC & 1 & No (?) & NO & MHD stability & $\begin{array}{l}\text { Demonstrated } \\
\text { high initial beta } \\
\text { in a relatively } \\
\text { simple } \\
\text { configuration }\end{array}$ & Moderatc \\
\hline Diffuse $Z$ pinch & 1 & YES & YES & $\begin{array}{l}\text { No equilibrial } \\
\text { with closed } \\
p=\text { const } \\
\text { surfaces; no } \\
\text { alpha } \\
\text { confinement }\end{array}$ & $\begin{array}{c}\text { Simplicity of the } \\
\text { configuration } \\
:\end{array}$ & Small \\
\hline Spheromak & 0.1 & YES & No & MHD stability & $\begin{array}{l}\text { Prosence of } \\
\text { intighetic } \\
\text { surfices }\end{array}$ & Moderate \\
\hline Spherical tokamak & 0.5 & YES & YES & $\begin{array}{l}\text { Difficult to creale } \\
\text { initial } \\
\text { configuration }\end{array}$ & $\begin{array}{l}\text { Good MFD } \\
\text { stibllity and } \\
\text { presertice of } \\
\text { niagh. surfaces }\end{array}$ & Large \\
\hline RFP & 0.1 & YES & YES & $\begin{array}{l}\text { Small beta; } \\
\text { complex } \\
\text { geometry of the } \\
\text { implosion }\end{array}$ & $\begin{array}{l}\text { Tnteresting } \\
\text { fusion-rclated } \\
\text { physics }\end{array}$ & Large \\
\hline Mirror & 1 & YES & NO & End losses & $\begin{array}{l}\text { Dingnostic } \\
\text { access from the } \\
\text { cuds }\end{array}$ & Large \\
\hline
\end{tabular}


The Disposable MITL Approach to Z-pinch Fusion Energy

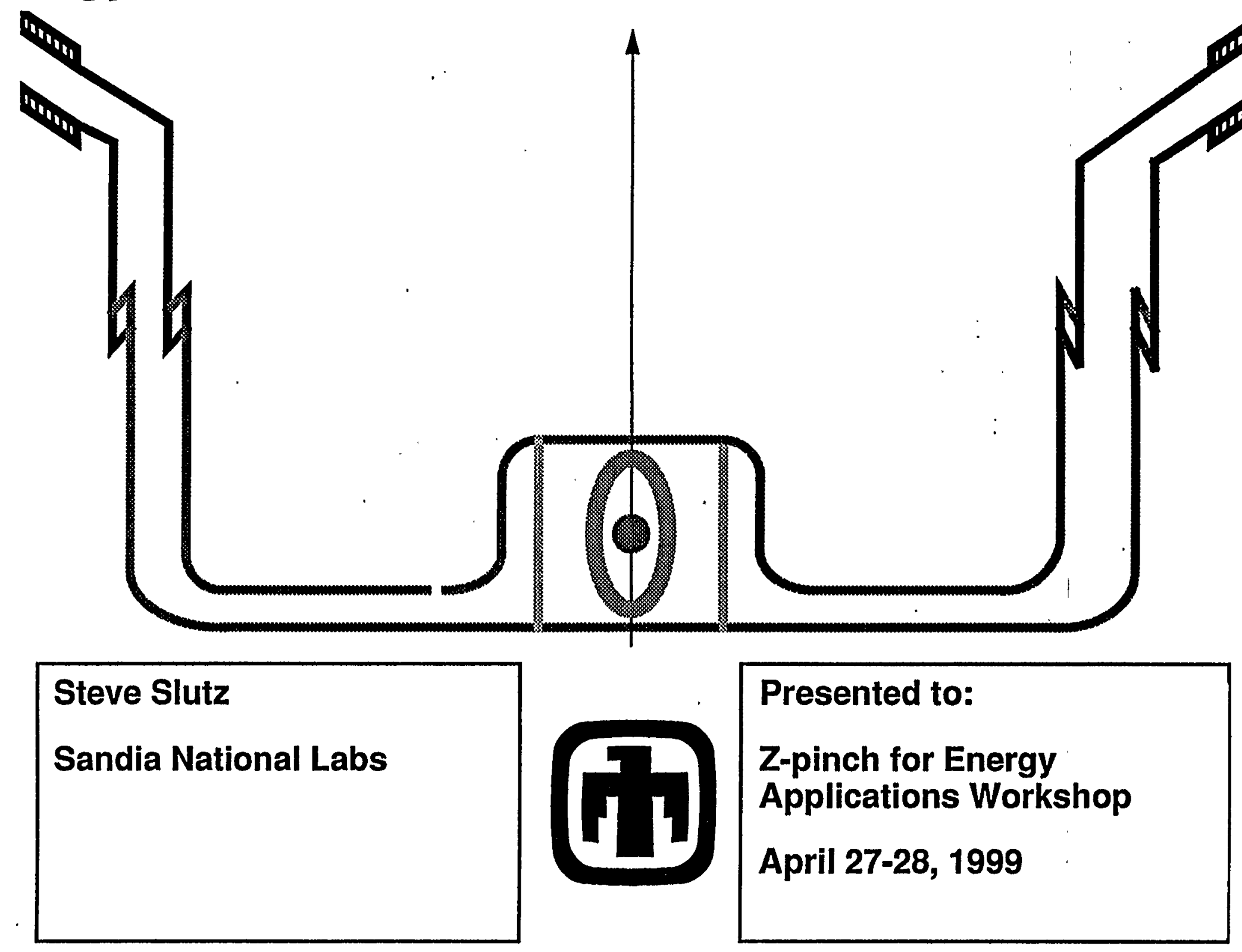




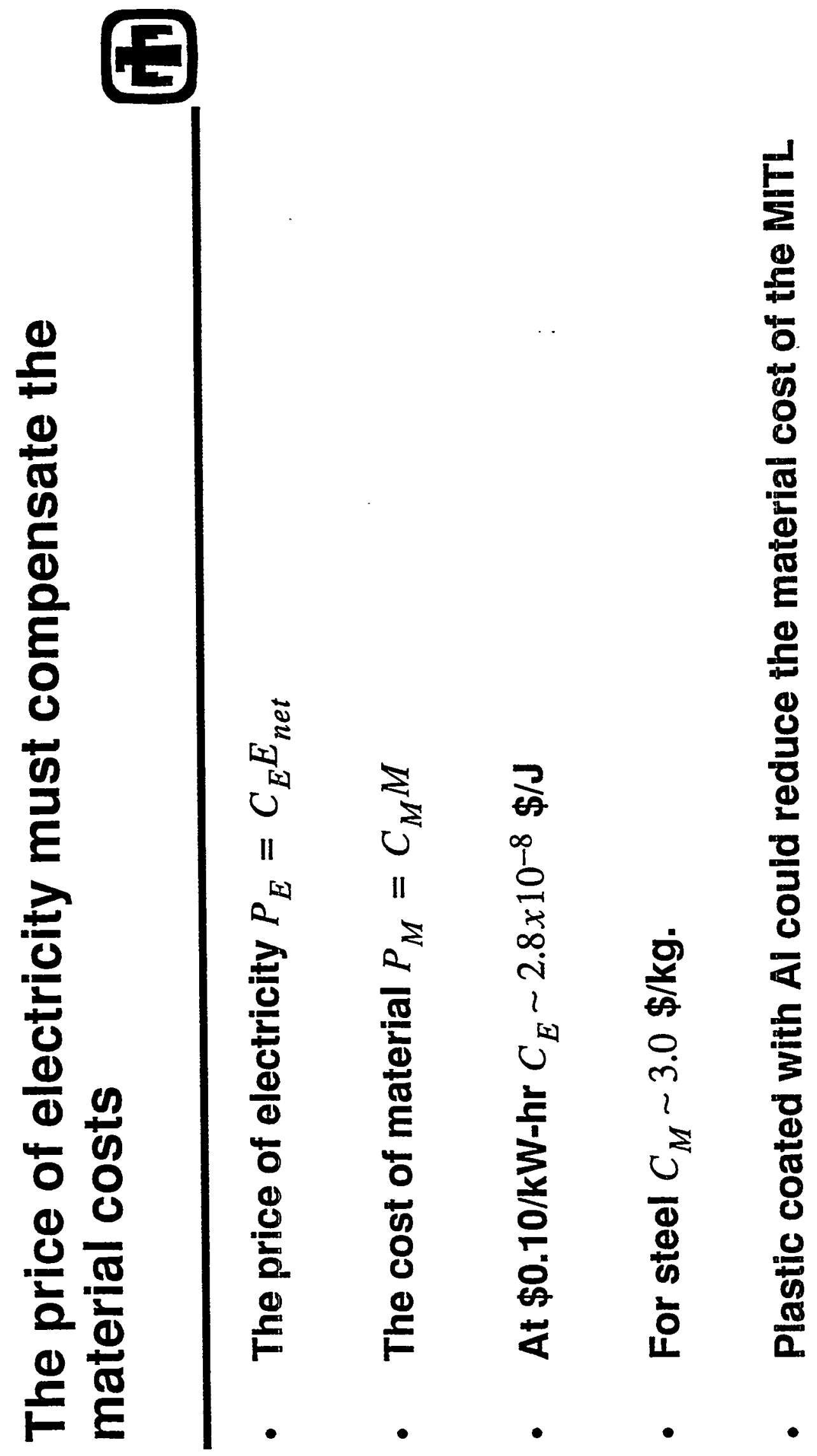




\section{Simple energy accounting determines the minimum capsule yield}

- The minimum capsule yield is given by the expression

$$
\varepsilon_{g} Y_{\text {min }}=\frac{E_{\text {mach }}}{\varepsilon_{m}}+\frac{C_{M} M}{C_{E}}
$$

- The net energy delivered by the accelerator is given by the equation

$$
E_{\text {mach }}=\left(1-\varepsilon_{c o n}\right)\left[W-\left(E_{p}+E_{k}\right)\right]+E_{p}+E_{k},
$$

- where $W$ is the total energy delivered in the pulse, $\varepsilon_{c o n}$ is the efficiency that excess magnetic energy can be converted back to electricity, $E_{p}$ is the energy delivered to the pinch, and $E_{k}$ is the kinetic energy of the MITLs due to magnetic pressure during the pulse. 


\section{Magnetic insulation determines the disk MITL gap and radius}

- Efficient power flow has been demonstrated with a gap $\left(d_{0}=2 \mathrm{~mm}\right)$ near the z-pinch.

- We shall assume a fixed gap in a disk MITL out to a radius $r_{0}$. Magnetic insulation determines the maximum value of $r_{0}$

- Magnetic insulation determines a critical voltage $V_{c}=B d_{0} c=\frac{\mu_{0} I}{2 \pi r_{0}} d_{0} c$, where $I=I_{\text {peak }} \sqrt{F_{L}}$ and $F_{L}$ is the fraction of the current lost.

- The voltage across the disk MITL is $V_{d}=\left(L_{p}+L_{d}\right) \frac{I_{\text {peak }}}{t_{\text {rise }}}$, where $L_{p}=\frac{\mu_{0}}{2 \pi} h_{p} \ln \left(\frac{d_{0}+r_{i}}{r_{f}}\right)$ and $L_{d}=\frac{\mu_{0}}{2 \pi} d_{0} \ln \left(\frac{r_{0}}{r_{i}}\right)$. 


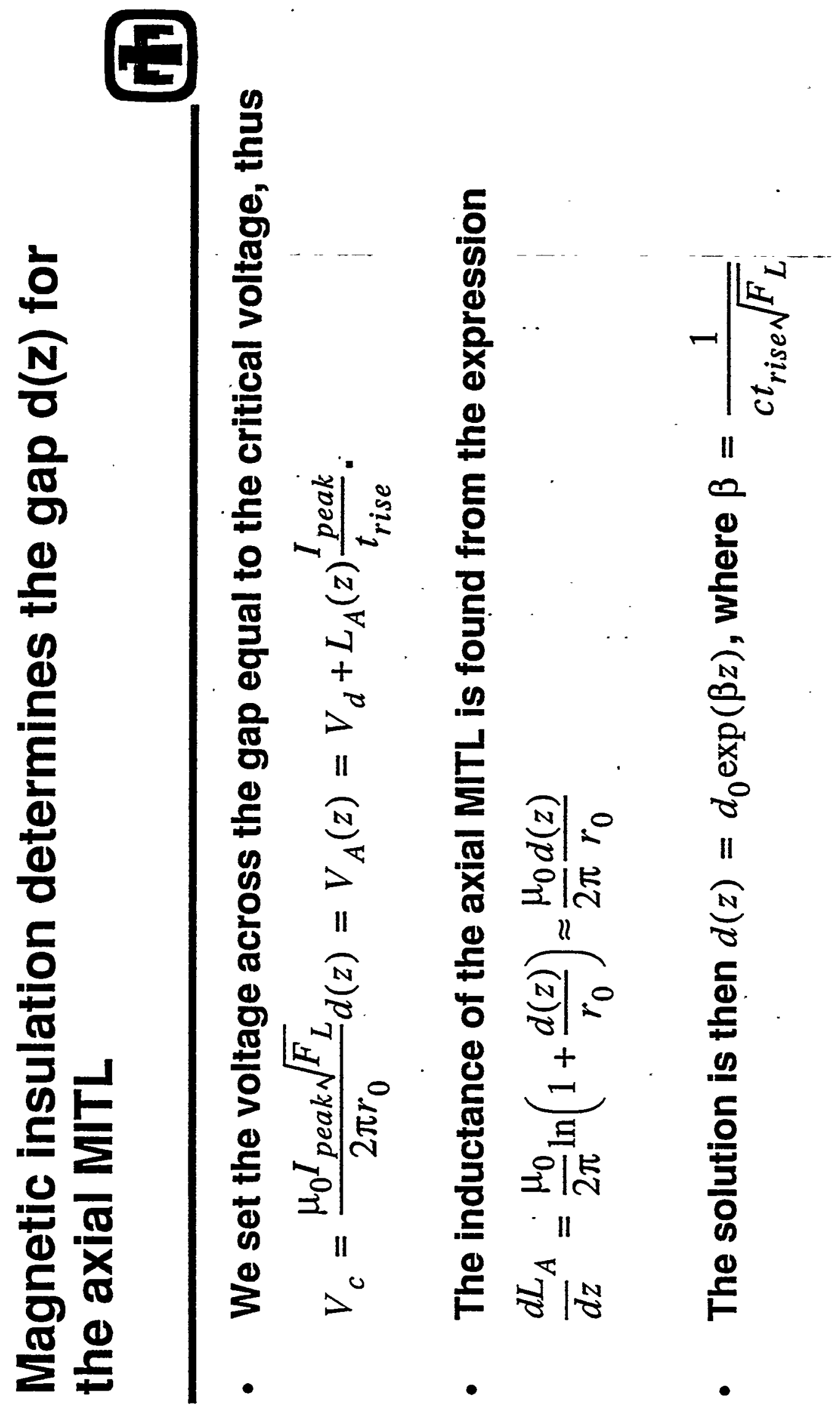




\section{The pinch energy and MITL kinetic energy can be determined analytically}

- The pinch radius is given by the expression $r=r_{i}\left(1-\tau^{4}\right)$ for the current profile $I^{2}(\tau)=\frac{3 \sqrt{3}}{2} I_{\text {peak }}^{2} \tau^{2}\left(1-\tau^{4}\right)$, where $\tau=\frac{t}{t_{p}}$.

- The pinch energy is then given by the expression $E_{p}=\sqrt{3} h_{p} \frac{\mu_{0}}{4 \pi} I_{p e a k}^{2}$, where $h_{p}$ is the height of the pinch.

- The motion of the MITL electrodes is given by the equation $\sigma \frac{d^{2} x}{d t^{2}}=\frac{B^{2}}{2 \mu_{0}}$, with the results $E_{k}=E_{0}\left(\frac{L_{\text {mitl }}}{r_{0}}+\ln \left(\frac{r_{0}}{r_{i}}\right)\right)$, where $E_{0}=\frac{3}{\pi^{3} \sigma_{0}}\left(\frac{\mu_{0} t_{p} I_{\text {peak }}}{28 r_{0}}\right)^{2}$ and we have assumed $\sigma(r)=\sigma_{0}\left(\frac{r_{0}}{r}\right)^{2}$ within the disk. 
The electrical energy is determined by the total inductance

- The energy supplied by the machine is given by the formula

$$
W(t)=\int_{0}^{t} V I d t=\int^{t} I \frac{d}{d t}(I L) d t=\frac{1}{2} L(t) I^{2}(t)
$$

- We define $W=\max (W(t))$

- The inductance rises due to pinch and electrode motion past the point of peak current. For simplicity we take the final inductance and peak current to calculate $\mathbf{W}$. 


\section{The motion of the electrodes increases the MITL inductance}

- The deflection of the MITLs is given by $x(\tau)=\frac{\alpha \tau^{4}}{12}\left(1-\frac{3 \tau^{4}}{14}\right)$, where

$\alpha=\frac{3 \sqrt{3} \mu_{0}}{16 \pi^{2} \sigma_{0}}\left(\frac{I_{p e a k} t_{p}}{r_{o}}\right)^{2}$

- The disk MITL inductance is $L_{d}=\frac{\mu_{0}}{2 \pi}\left(d_{0}+x(\tau)\right) \ln \left(\frac{r_{0}}{r_{i}}\right)$

- The axial MITL inductance is $\frac{\mu_{0}}{2 \pi r_{0}}\left[x(\tau) L_{m i t l}+d_{0}\left(\frac{\exp \left(\beta L_{\text {mitl }}-1\right)}{\beta}\right)\right]$ 
The minimum yield is a strong function of the standoff distance

- The optimum MITL areal density

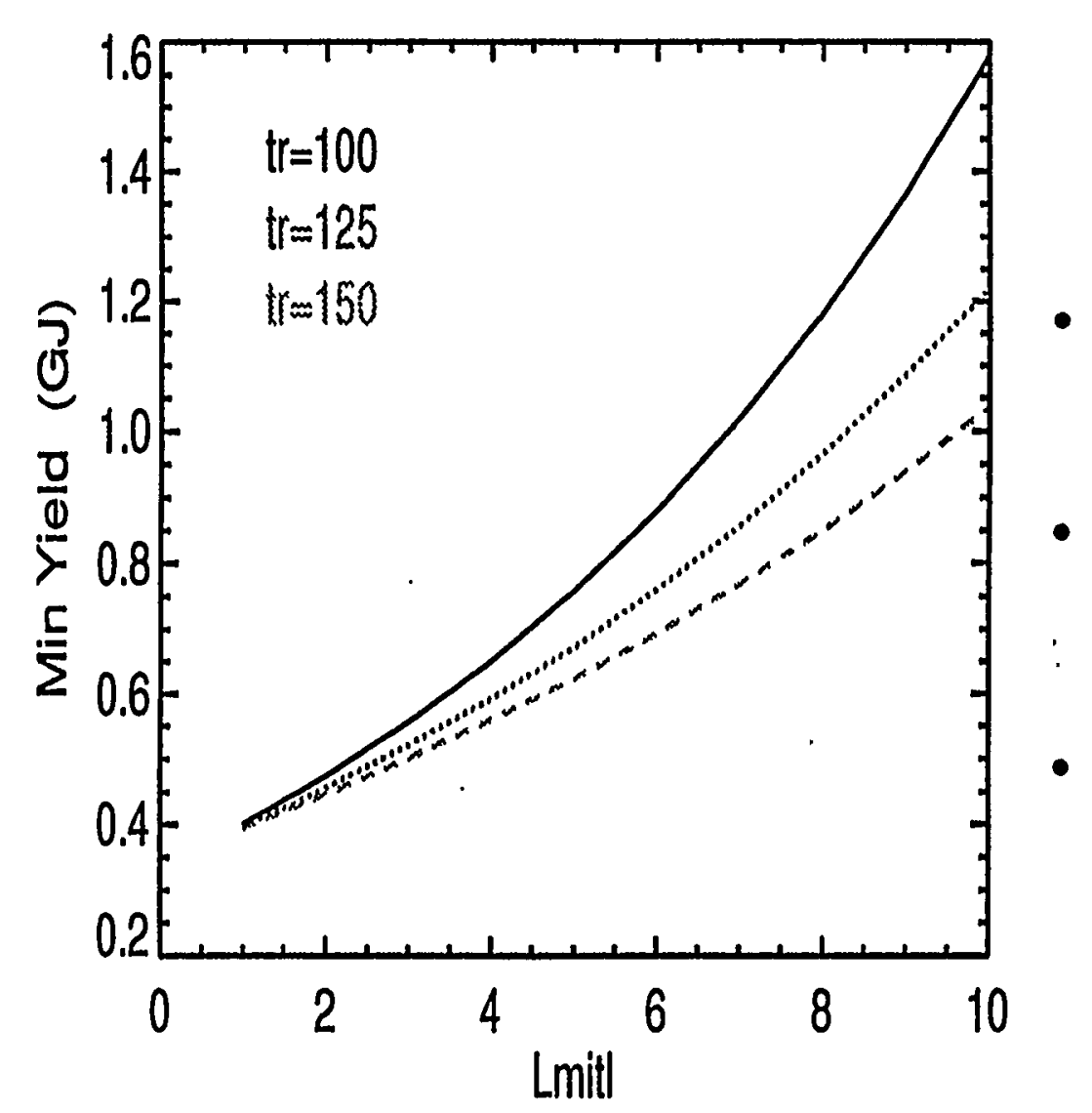
is $(7.7,6.3,5.4) \mathrm{mg} / \mathrm{cm}^{2}$ for $t_{\text {rise }}=(100,125,150)$ and nearly independent of $L_{\text {mitt }}$

Electrical conversion efficiency has been assumed to be $50 \%$

No excess power is returned to the accelerator, i.e. $\varepsilon_{c o n}=0$

The machine efficiency is assumed to be $10 \%$. 


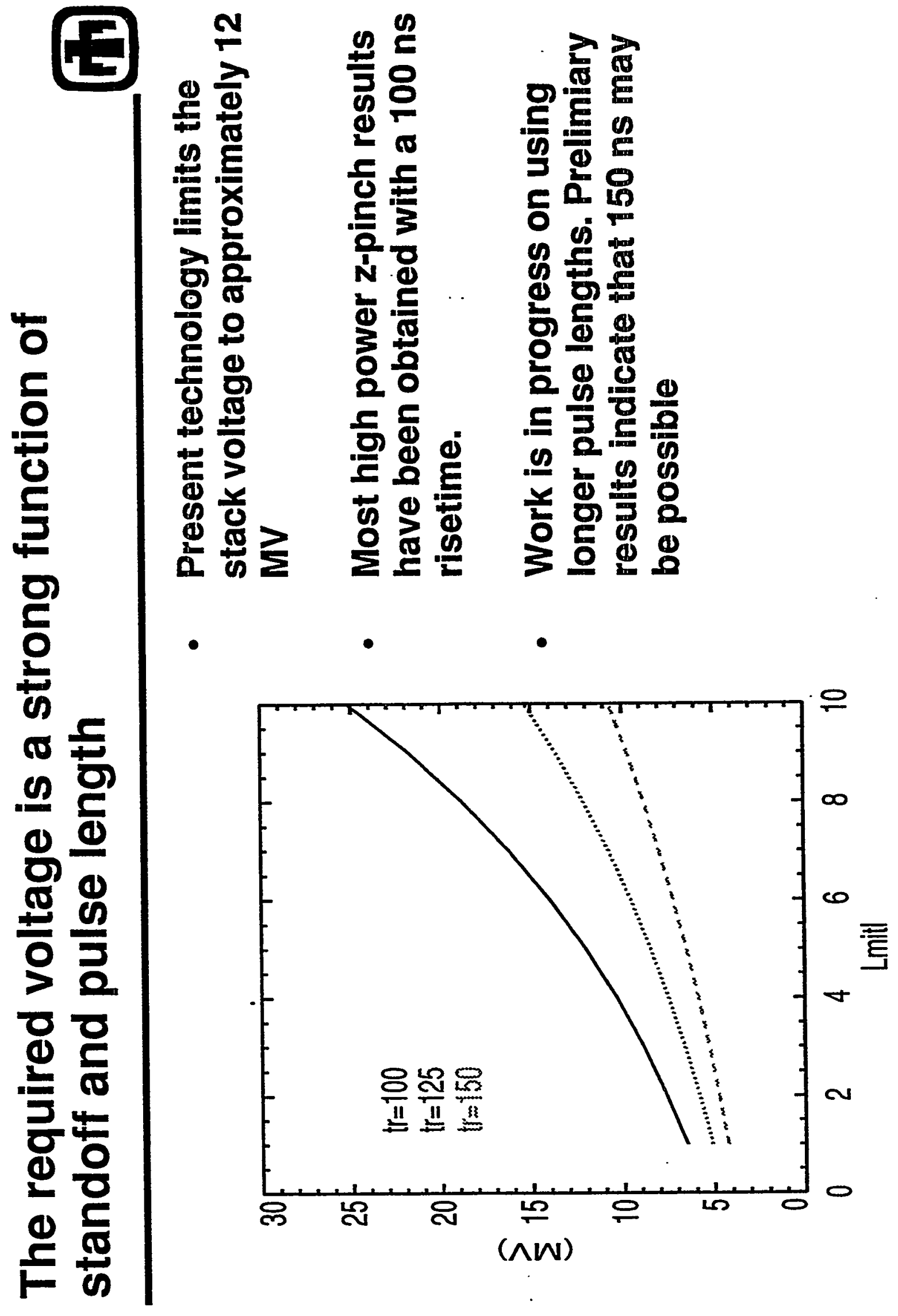




\section{Converting reflected power back into electrical energy significantly lower the minimum yield}

- Converting $50 \%$ of the excess magnetic energy reduces the

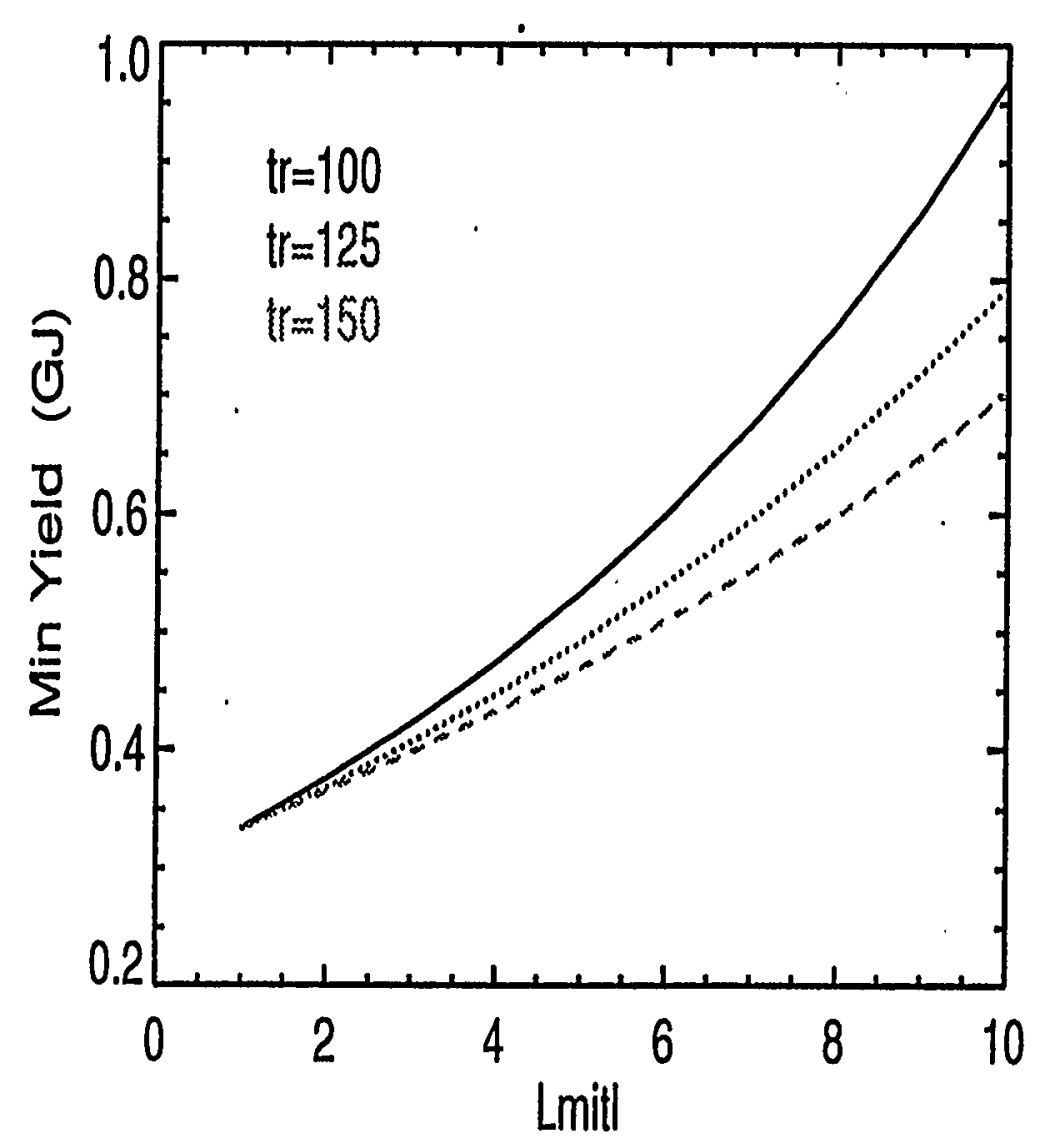
minimum yield by approximately $60 \%$.

- This conversion could be accomplished in principle by recharging capacitors with the reflected wave.

- Details need to be worked out. 


\section{Summary}

- Minimum yields have been calculated based on a disposible MITL

- The minimum yields are of order $1 \mathrm{GJ}$

- An efficient system would need higher yields.

- The shot frequency should be less than 1 hertz

- Standoff distances of greater than $4 \mathrm{~m}$ will be required

- Such a system may not be unreasonable!! 
Appendix B - Literature Search 


\section{Z-Pinch Reactor Study Bibliography}

Papers are listed from newest to oldest.

1. L. John Perkins, 'The role of inertial fusion energy in the energy markeplace of the $21^{\text {st }}$ century and beyond', Nucl. Instr. \&Meth. in Phys. Res. A 415, 44-60 (1998).

An IFE reactor can achieve credible capacity factors, because its power core is comparable in complexity to a fission reactor vessel, with additional environmental advantages.

2. L. John Perkins, 'Complexity and Availability for Fusion Power Plants: The Potential Advantages of Inertial Fusion Energy', Journal of Fusion Energy 16, 307-316 (1997).

The 'CRAM' concept - complexity, reliability, maintainability, availability. Discussion of actual fission reactor duty cycles. Advantage of MFE over IFE due to: 1) decoupling of driver and thermal conversion system, 2) liquid walls, and 3) multiplexing of reactor chambers.

3. L. J. Perkins et al, 'High Density, High Magnetic Field Concepts for Compact Fusion Reactors', Proc. Of $16^{\text {th }}$ Int'l IAEA Conf. On Fusion Energy, Montreal, 7-11 Oct, 1996, IAEA-CN-64/GP-18, pp. 619-625.

Discussion of compact reactor design for: staged Z-pinch, liner implosion of closed field line configurations, magnetic 'fast' ignition of inertial fusion targets, continuous $\mathrm{Z}$ pinch.

4. L. John Perkins, "The Restructured Fusion Program and the Role of Alternative Fusion Concepts', Testimony to the U.S. House of Representatives Committee on Science, Lawrence Livermore National Laboratory Report, UCRL-ID-123417 (1996).

About $25 \%$ of the fusion budget should be devoted to alternative concepts, principally IFE, the emphasis should be on science-based study of alternatives rather than engineering the present conventional route, and reactor operation cheaper than the mainline tokamak approach should be the ultimate criterion.

5. Ralph W. Moir, 'IFE Power Plant Design Strategy', Fusion Tech. 30, 1613-1623 (1996). Costing study of Heavy-Ion-based power plant. Refinement of previous studies.

6. H. U. Rahman, F. J. Wessel and N. Rostoker, 'Staged Z Pinch', Phys. Rev Lett. 74, 714-717 (1995).

7. B. Grant Logan, Ralph W. Moir, and Myron A. Hoffman, 'Requirements for low-cost electricity and hydrogen fuel production from multiunit inertial fusion energy plants with a shared driver and target factory', Fusion Tech. 28, pp. 1674-1696 (1995).

Systems study based on HYLIFE-II heavy-ion based reactor concept. RHEPP-1 cited.

8. A. E. Robson, 'Prospects for Fusion with Dense Z-Pinches', Proc. Third Int'l Conf on Dense

Z-Pinches, London, 1993, M. Haines and A. Knight, Eds., pp 707-715 (1994).

Continuation of previous work - pinch assumed stable on the current rise, zero-D modeling. Design for $40 \mathrm{~Hz} 100 \mathrm{MW}$ (e) DZP reactor, where pinch is formed between two liquid Li jets.

9. Thomas J. Dolan, 'Fusion Power Economy of Scale', Fusion Tech. 24, pp. 97-111 (1993). 
General discussion (political/economic) of comparisons between fusion-based and other power plants.

10. R. W. Moir et al, 'HYLIFE-II - A molten-salt inertial fusion energy power plant design final report', Fusion Tech. 25, 5-25 (1994)

11. B. Grant Logan, 'Inertial fusion reactors using Compact Fusion Advanced Rankine (CFARM) MHD conversion', Fusion Eng. and Design. 22, 151-192 (1993).

12. A. E. Robson, 'Physics of a Dense Z-Pinch Fusion Reactor', Varenna 1990, pp. 1-15.

13. H. R. Bolton, P. Choi, A. E. Dangor, A. J. H. Goddard, M. G. Haines, S. J. Peerless, A. Power, and S. P. Walker, 'The dense Z-Pinch as a fusion reactor: a first technical appraisal', Fusion Eng. And Design. 10, pp. 9-13 (1989).

Based on prior Bolton, Robson, Hartmann work, emphasizing pulsed power aspects of reactor design, electrode heating and erosion, reactor energetics, $50 \mathrm{~Hz}$ operation mentioned $(80 \mathrm{MW})$.

14. A. E. Robson, 'Physics of a Dense Z-Pinch Fusion Reactor', Second Int'l Conf on Dense ZPinches, Laguna Beach, pp. 362-375 (1989).

Continuation of previous work.

15. A. E. Robson, 'Evolution of a z-pinch with constant dI/dt', Nuclear Fusion 28, pp. 2171-2178 (1988). Different emphasis from his other papers. It is shown that there is maximum current in $\mathrm{H}$ or $\mathrm{D}$ equal to $\sim 3.5$ times the Pease-Braginskii current which cannot be exceeded because of radiative collapse.

16. A. J. Power and H. R. Bolton, Repetitive Pulsed Power Systems for a Dense Z-Pinch Reactor', DZP/P47, Dec 18, 1988.

17. A. J. Power, H. R. Bolton, and W. Bessel, 'Simulation of a Pulsed Power Scheme feeding a Dense Z-Pinch Plasma Load', Proc. IEEE Pulsed Power Conf., pp. 696-699 (1987).

18. S. P. Walker and M. Javadi, 'The dense Z-Pinch as a fusion power reactor: pressure vessel integrity and power density considerations', Fusion Eng. and Design. 5, pp. 261-267 (1987).

19. M. G. Haines and S. P. Walker, 'Compact fusion reactors: the potential of the dense $Z$ pinch', Nucl. Energy 26, pp. 362-375 (1987). Good overall paper detailing physics issues, as well as summaries of key earlier Hartman, Hagenson, Robson, and Bolton.

20. H. R. Bolton et al, 'Engineering study of the feasibility of a fusion power reactor employing the dense Z-pinch plasma confinement geometry', Proc. $11^{\text {th }}$ Int'l Conf. on Plas. Phys. And Controlled Fusion Res., Kyoto, Nov. 13-20, 1986, pp. 367-372.

21. S. P. Walker, M. Javadi, and A. E. Robson, 'The Dense Z Pinch as a Fusion Power Reactor: Breeding and Radiation Damage Considerations', Proc. $14^{\text {th }}$ Symp. On Fusion Technology, Avignon, pp. 1875-1880 (1986). Neutron fluxes to first wall need to be low as in a pressurized water reactor (PWR), but still results in power densities two orders of magnitude higher than tokamaks. 
22. J. A. Heikkinen, S. J. Karttunen, and R. R. F. Saloma, 'Feasibility Studies of fast electron driven dense Z-pinch fusion reactor', Proc. $4^{\text {th }}$ Int'l Conf. On Emerging Nuclear Energy Systems ( $4^{\text {th }}$ ICENES), Madrid, June 30-July 4, 1986. Use of a $<1 \mathrm{~kJ}$ laser to initiate fast electron current-driven Z-pinch discharge.

23. H. R. Bolton et al, 'The dense Z-Pinch: a first technical appraisal', Centre for Fusion Studies, Imperial College, London, Final Report on SERC Grant GR/C/80066 (evidently 1986). Long version of paper no. 18 above.

24. R. R. Peterson, 'Repetition rates in heavy ion beam driven fusion reactors', Heavy Ion Inertial Fusion, AIP Conf. Proc. 152, Washington, DC, pp. 507-514 (1986). Limitations to havey-ion beam rep-rate caused by wait-time to clear out vaporized material from target chamber.

25. A. E. Robson, 'The dense Z-Pinch as a fusion reactor', Proc. Conf. Dense Z-Pinches for Fusion, Alexandria, VA, March 29-30, 1984, J. Sethian and K. A. Gerber, eds., pp. 2-14.

26. F. Winterberg, 'Inertially stabilized thermonuclear Z-Pinch', Atomkernenergie-Kerntechnik 43, pp. 31-36 (1983).

27. M. G. Haines, 'The Physics of the Dense Z-Pinch in Theory and in Experiment With Application to Fusion Reactor', Physica Scripta T2/2, pp. 380-390 (1982).

28. M. G. Haines, 'The high density Z-Pinch as a fusion reactor', in 'Alternative Approaches to Fusion', Plenum Press (1982).

29. A. G. Fischer, 'The Fiscatron fusion reactor concept', in Kerntechnik, 4, pp. 182-184 (1978).

30. R. L. Hagenson, A. S. Tia, R. A. Krakowski, and R. W. Moses, 'The dense Z-Pinch (DZP) as a fusion power reactor: preliminary scaling considerations and systems energy balance', Nucl. Fusion 21, pp. 1351-1361 (1981).

Key early reference.

31. Same as Ref. 30, LANL Report LA-8186-MS

Pre-submittal to Nuclear Fusion.

32. Halil I. Avci and Gerald L. Kulcinski, "The effect of liquid-metal protection schemes in inertial confinement fusion reactors, Nucl. Technology 44, pp. 333-345 (1979).

33. C. W. Hartman, G. Carlson, M. Hoffman, R. Werner, D. Y. Cheng, 'A Conceptual Fusion Reactor based on the high-plasma-density Z-Pinch', Nucl. Fusion 17, pp. 909-918 (1977).

Key early reference.

34. Jay E. Hammel, ‘An Ohmically heated high-density Z Pinch', LANL Report LA-6203-MS, (1976).

35. R. S. Pease, 'Equilibrium Characteristics of a Pinched Gas Discharge Cooled by Bremsstrahlung Radiation', Proc. Phys. Soc. Xxx, pp. 11-23 (1957). 
36. R. S. Pease, 'Equilibrium Characteristics of a Pinched Gas Discharge Cooled by Bremsstrahlung Radiation', Proc. Phys. Soc. Xxx, pp. 11-23 (1957). 


\title{
Appendix C - Snowmass Fusion Summer Study (July 11-23, 1999)
}

\author{
Snowmass Fusion Summer Study (July 11-23, 1999) \\ Inertial Fusion Concepts Working Group Final Report
}

Craig Olson, Max Tabak, Jill Dahlburg, Rick Olson

Steve Payne, John Sethian, John Barnard, Rick Spielman

.. . Ken Schultz, Robert Peterson, Per Peterson.

Wayne Meier, John Perkins

\section{Overview (Craig Olson)}

The Inertial Fusion Concepts Working Group was organized around the Snowmass theme of examining issues and opportunities for the next decade. Organization of this working group began almost a year before the Snowmass meeting, when it was decided to structure this working group into four Subgroups as follows:

(1) Targets

(2) Drivers and Standoff

(3) Inertial Fusion Power Plants

(4) IFE Metrics and Development Paths

To guide the discussions, a series of key questions, or "hot topics" was developed. The purpose of these questions was to establish the status and issues of the various areas of IFE, and examine how existing (and possibly new) facilities could be used to address these issues during the next decade. A complete listing of the Convenors, Subgroup Leaders, and Session Leaders is given in Table 1. A complete listing of the "hot topics" questions is given in Table 2.

The format of this Working Group's Sessions was to have open discussions. During the first week, the first three Subgroups had parallel Sessions. Each Session had one Session Leader and spent a morning (three and a half hours) discussing the answer to one "hot topics" question. Speakers were invited to give short presentations to initiate the discussions. Each speaker was asked to give a concise summary of the present status of the particular issue in question, and then propose a "strawman" answer to the question to initiate discussion. The discussion period after each talk was typically as long as, or longer than, the talk. In addition, if anyone wanted to give a relevant, brief talk, they were invited to contact the Session Leader, who arranged it in the schedule of their Session. There were no predetermined conclusions. It was hoped that consensus would be reached in most cases. However, if there were clearly diverging opinions, then it was agreed that both sides should be presented in this final report. The Session Leader was

responsible for coordinating the written answer to their Session's question, with input from speakers, contributors, and all participants. At the end of the first week, and during the second week, the entire Inertial Fusion Concepts Working Group met in plenary session to discuss the Subgroup 4 Topics, and then to continue discussions from the first week on some of the more difficult issues.

The summaries of the four Subgroups and of the eleven "hot topics" Sessions form the core of this report. Before presenting these detailed results (which are given in the following sections), we would first like to give an overview as to how all of the inertial fusion elements should fit together during the next decade, and then give brief summaries of each Subgroup. Lastly, brief summaries are given of the discussions on "special issues." 


\section{Table 1. Inertial Fusion Concepts Working Group}

Organizer: Craig Olson

Convenors: John Barnard, John Lindl, Craig Olson, Steve Payne, John Sethian, Ken Schultz, Rick Spielman

Subgroup Leaders:

(1) Targets: $\quad$ Max Tabak, Jill Dablburg,

Rick Olson

Steve Payne, John Barnard,

(2) Drivers \& Standoff: $\quad$ Steve Payne, John Barnard, John Sethian, Rick Spielman

(3) Power Plant Concepts: Ken Schultz, Robert Peterson,

Per Peterson

(4) Metrics \& Pathways: Wayne Meier, John Perkins

Question 1A : Max Tabak

Question 1B: Jill Dahlburg

Question 1C: Rick Olson

Question 2A: John Sethian

Question 2B: John Barnard

Question 2C: Rick Spielman

Question 3A: Robert Peterson

Question 3B: Per Peterson

Question 3C: Ken Schultz

Question 4A: John Perkins

Question 4B: Wayne Meier

Table 2. "Hot Topics" Questions.

Targets

(1A) What are the key scientific issues for validating each target concept, and how can they be resolved?

(1B) How can existing (and new?) facilities be used to test each concept?

(1C) What IFE target physics issues will not be resolved on NIF? What is required to get to high yield? What is the significance to IFE of experimentally demonstrating high yield/high gain?

Drivers and Standoff

(2A) How can the source brightness, beam uniformity, pulse shaping accuracy, efficiency, reliability, repetition rate, and cost of each driver concept be improved?

(2B) What are the key standoff issues for each driver scenario and how can they be addressed? (e.g., final optics for lasers, final transport and focus for laser beams, final focus magnetic lenses for heavy ion beams, power feed for rep-rated $z$ pinches,...)

(2C) What would convincingly demonstrate that each driver concept is a viable driver candidate for IFE? Specifically, what is a convincing Integrated Research Experiment (IRE) for each driver candidate?

Inertial Fusion Power Plant Concepts 
(3A) What are the key IFE power plant concepts, advantages, and issues?

(3B) What are the key scientific issues for the fusion chamber (e.g., first wall protection, ...), and what are the proposed solutions? What experiments could be done to test the relative merits of these solutions?

(3C) What are the issues in target fabrication, target characterization, target injection, target robustness (e.g., tolerances), and what is the path for addressing them?

IFE Metrics and Development Paths

(4A) What are the metrics for an entire IFE system for each step of development (e.g., concept exploration, proof of principle, performance extension, fusion energy development, DEMO, attractive commercial fusion power plant)? How are these incorporated into the IFE Road Map? How do we insure that there is a mechanism in place for new concepts to Initiate a development path?

(4B) What is the status and development path of each present IFE scenario?

\section{IFE Overview}

An IFE power plant is an integrated choice of:

(1) Target (direct drive, indirect drive, etc.) :

(2) Driver (heavy ion, KrF laser, DPSSL laser, z-pinch, light ion, etc.)

(3) Chamber (dry wall, wetted wall, liquid wall, etc.)

(4) Power conversion (Rankine, Brayton, etc.)

The separation of the power plant into four separate areas offers unique advantages for the inertial fusion approach to energy. Within certain constraints; it allows one to optimize the choice from each category to make the best integrated choice for any particular driver. Presently, there are two mainline approaches to inertial fusion energy heavy ions and lasers. The present preferred heavy ion scenario uses an induction-linac heavy-ion driver, an indirect-drive target; and a liquid-wall or wetted-wall chamber. The laser scenario uses either a Krypton Fluoride $(\mathrm{KrF})$ laser driver or a Diode-Pumped Solid State Laser (DPSSL) driver, a direct-drive target, and a dry-wall chamber. Other driver concepts also exist as exploratory concepts: a z-pinch scenario would use a pulsed power z-pinch driver, an indirect drive target, and a liquid wall or possibly solid Li with voids and a density gradient; a light ion scenario would use an ion diode driver, an indirectdrive target, and a wetted-wall chamber. Other exploratory concepts include the Fast Ignitor target concept, and Magnetized Target Fusion (MTF), which is between IFE and MFE.

An overview of the present IFE program is given in Table 3, organized according to drivers. The mainline approaches include heavy ions and lasers: these are at the Proof of Principle (PoP) stage, and are progressing toward the Performance Extension (PE) stage. Also, at the Concept Exploration (CE) level are z pinches, light ions, and MTF. In addition to entries for drivers, targets and power plants, note the entries for stand-off issues. Standoff refers to the interface between the driver and the target, and involves specific chamber issues associated with, e.g., final optics or final transport in the chamber. While science was the main charter of this Snowmass meeting, the FY99 funding levels for IFE are also given in Table 3, to show the present scale of these programs. In the last column, aspirations for the next decade are given. The three mainline approaches are currently at the PoP level: each desires a funding level of about $\$ 16 \mathrm{M}$ for 4-5 years, following which a decision would be made to as to whether to proceed on to the PE level. The Integrated Research Experiment (IRE) is at the PE level and would cost of the order of $\$ 50 \mathrm{M}-\$ 150 \mathrm{M}$. It is suggested that a decision should be 
made in 4-5 years (given adequate funding during that time) to proceed with 0,1 , or more IRE's. In addition, CE level support is needed for the next several years for $\mathrm{z}$ pinches,

Table 3. IFE Overview.

\begin{tabular}{|c|c|c|c|c|c|c|}
\hline Approach & Driver & Target & $\begin{array}{l}\text { Standoff } \\
\text { Issue }\end{array}$ & $\begin{array}{l}\text { Power } \\
\text { Plant } \\
\text { Concept }\end{array}$ & $\begin{array}{l}\text { FY99 } \\
\text { Funding } \\
\text { for IFE }\end{array}$ & $\begin{array}{l}\text { Aspirations for } \\
\text { Next Decade }\end{array}$ \\
\hline $\begin{array}{l}\text { Main-line } \\
\text { approaches } \\
\text { (from PoP } \\
\text { to PE) }\end{array}$ & $\begin{array}{c}\begin{array}{c}\text { Ion -heavy ion } \\
\text { induction } \\
\text { linac } \\
\text {-DPPSL }\end{array} \\
\text { Laser } \\
\text {-KrF }\end{array}$ & $\begin{array}{l}\begin{array}{l}\text { Indirect- } \\
\text { drive }\end{array} \\
\text { Direct-drive } \\
\text { Direct-drive }\end{array}$ & $\begin{array}{l}\text { Ton beam } \\
\text { Transport } \\
\text { Final optic } \\
\text { Final optic }\end{array}$ & $\begin{array}{l}\begin{array}{l}\text { Liquid } \\
\text { wall }\end{array} \\
\text { Dry wall } \\
\text { Dry wall }\end{array}$ & $\begin{array}{l}\$ 8 \mathrm{M} \\
\$ 4 \mathrm{M} \\
\$ 8 \mathrm{M}\end{array}$ & $\begin{array}{l}\text { Each program: } \\
4-5 \text { years research* } \\
\text { at } \sim \$ 16 \mathrm{M} / \text { year } \\
\text { leading to an } \mathrm{REE} \\
\text { for } \sim \$ 50 \mathrm{M}-\$ 150 \mathrm{M} \\
(0,1, \text { or more IRE's })\end{array}$ \\
\hline $\begin{array}{l}\text { Concept } \\
\text { Exploration } \\
\text { (CE) }\end{array}$ & $\begin{array}{l}\text { Z-pinch } \\
\text { Ion-light ion } \\
\quad \text { diode } \\
\text { Magnetized } \\
\text { target fusion }\end{array}$ & $\begin{array}{l}\text { Indirect- } \\
\text { drive } \\
\text { Indirect- } \\
\text { Drive } \\
\text { Magnetized } \\
\text { plasma }\end{array}$ & $\begin{array}{l}\text { Recyclable } \\
\text { transmission } \\
\text { line } \\
\text { Ion beam } \\
\text { transport } \\
\text { Recyclable } \\
\text { transmission } \\
\text { line }\end{array}$ & $\begin{array}{l}\text { Solid Li } \\
\text { Wetted } \\
\text { Wall } \\
\text { Solid Li }\end{array}$ & $\begin{array}{l}\$ 0.2 \mathrm{M} \\
0 \\
\$ 1 \mathrm{M}\end{array}$ & $\begin{array}{l}\text { Investigate concept } \\
\text { and rep-rate } \\
\text { Science-level ion } \\
\text { source } \\
\text { development } \\
\text { PoP experiment } \\
(-\$ 21 \mathrm{M} / 3 \text { years })\end{array}$ \\
\hline
\end{tabular}

environmental attractiveness,...

*includes chamber, target development,

light ion sources, and MTF (as well as for the fast ignitor target concept, and new IFE concepts). Note that the current desired funding includes support for chamber development and target development.

As discussed in the summaries to follow, issues for target physics will be addressed mainly with existing facilities during the next decade. Specifically, target development (indirect-drive and direct-drive), ignition, and the start of propagating burn will be studied on Omega, Nike, $Z$, and NIF (all of which are funded through DOE DP). It is important to note that it was envisioned at Snowmass that no new "target shooter" facilities will be required during the next decade, because NIF will be coming online. The question of if and when a high-yield/high-gain single-shot facility is needed was debated (see discussion below). For IFE, the main facilities envisioned for the next decade are one or more IRE's.

Brief summaries of the main points of discussion of the four Subgroups follow:

\section{Targets: .}

There are several key physics issues for targets that need to be resolved during the next decade. (Note: target fabrication and injection are covered under Subgroup 3 Inertial Fusion Power Plants.) For indirect drive, key issues are laser-plasma interaction, target gain, and capsule stability. For HIF indirect drive, the deposition profile and the hydrodynamic motion of the converter are of concern. For z-pinch indirect drive, the coupling efficiency, wire array stability, and the symmetry and temporal history of the radiation are key issues. For direct drive, the most critical issue is hydrodynamic stability, and ultimately, 3D integrated hydrodynamic calculations will be required. For the fast 
ignitor, coupling efficiency is the most critical issue. For MTF, the key issue is whether the $Q$ will be large enough for fusion energy.

Several facilities will be used to address these issues. NIF will be used to study gain energetics, pulse shaping and compression, symmetry for indirect drive, hydrodynamic instability for direct drive, and ignition and burn. OMEGA will be used to study spherical warm and cryogenic' pellets, direct drive cylinders, scale ' 1 ' hohlraums, planar targets, tetrahedral hohlraums, and shock-tube hohlraums. Nike will examine hydrodynamic instability effects, EOS measurements, and preheat effects. Z will be used to study hohlraum features (energetics, wall opacity, wall motion, hole closure), capsule ablator EOS, DT EOS, shock propagation in ablator materials and ablator burnthrough. GSI can provide a capability to obtain stopping power data relevant the HIF. Atlas and Shivastar can be used to study MTF.

Looking ahead to NIF and beyond, there was a discussion concerning what physics issues will not be resolved on NIF. For indirect drive, these included issues that can't be studied on NIF (e.g., ion deposition) or that are not in the current NIF plan (e.g., fast ignitor). For direct drive, issues that can't be studied included, e.g., high yield. Several possible approaches to high yield were discussed. For the $\mathrm{Z}$ approach, the path to $\mathrm{ZX} / \mathrm{X}-1$ would lead to high yield (LLNL has calculated yields of 400 -1000 MJ using the X-1 power pulse input). For indirect drive with glass lasers, assuming that an "advancedcoupling target" is feasible, it may be possible to do modest gain on NIF; recent 2D calculations give a $70 \mathrm{MJ}$ yield, and it is believed that this could be significantly higher. In general, it is believed that "bigger is easier" for targets. There was debate over whether or not a high-yield/high-gain, single-shot facility is needed for IFE development. Arguments against included, e.g., ignition and burn physics are scale-size invariant, and that high yield may be possible on NIF. Arguments for included, e.g., that DOE DP has a high yield mission need and might provide a $\sim \$ 1 \mathrm{~B}$ single-shot, high-yield facility, and that this would greatly reduce the risk for IFE development. The debate continued throughout Snowmass (see "special issues" below).

\section{Drivers and Standoff}

Presently, there are five driver candidates in IFE: heavy ions, $\mathrm{KrF}$ lasers, DPSSL's, $\mathrm{z}$ pinches, and light ions. Over the past two decades, heavy ions have been the primary approach to inertial fusion energy, because of their perceived high efficiency and excellent durability. In the last few years, $\mathrm{KrF}$ lasers and DPSSL's have also developed viable fusion energy programs. Very recently, a z-pinch approach to fusion energy has been proposed. The light ion approach, after a decade of research, is on hold - the key problem is the ion source, and it is suggested that a science-level program (e.g., at universities) to develop a light ion source might eventually leverage the past investment in this approach.

Standoff refers to the method used to separate the driver from the target. For ions, the standoff distance is typically of order 5 meters (distance from the target to the final focus magnet system); for lasers, the standoff distance (distance from the target to the final optic) is typically 25 meters. Ion beam standoff involves final transport and stability issues of beams in the fusion chamber. Heavy ion beams use vacuum transport or partially-neutralized transport, or any of several channel-like transport schemes. Light ion transport uses fully-neutralized transport, or any of several channel-like transport schemes. The use of high-current $(-100 \mathrm{kA})$ proton beams to model stripped heavy ion beams was advocated (since there are no high current heavy ion beams presently). For $\mathrm{KrF}$ or DPSSL's, the final optic may be a grazing incidence metal mirror; in addition, a hot fused silica wedge or grating may be used for DPSSL's. For z pinches, a recyclable transmission line (RTL) is being considered. All standoff scenarios need further development. 
IRE scenarios were discussed for each of the five driver scenarios. The heavy ion, $\mathrm{KrF}$, and DPPSL IRE's are the most developed. Approaches to IRE's for $\mathrm{z}$ pinches and light ions were also discussed. The key IRE issues (which are therefore critical opportunities for development) are in neutralized and channel-like transport for heavy ions; in durability for $\mathrm{KrF}$ (survivability of the pumping foil); in cost of diodes for DPSSL's; in durability for $\mathrm{z}$ pinches (ability to recycle the transmission line); and in durability for ion sources and final transport for light ions.

\section{Inertial Fusion Power Plant Concepts}

IFE power plant concepts were discussed, including studies for heavy ions, light ions, lasers (relevant to both $\mathrm{KrF}$ and DPSSL's), z pinches, and MTF. The mainline approaches use liquid walls or wetted walls for ions, consistent with indirect drive having a limited input solid angle; and dry walls or wetted walls for $\mathrm{KrF}$ or DPSSL's, consistent with direct drive having a large input solid angle. $Z$ pinches or MTF would use thick liquid walls or possibly solid Li with voids and a density gradient for shock dissipation.

Requirements for the IFE chamber were enumerated, and the relative advantages of dry wall, wetted wall, and thick liquid walls for various drivers were discussed. The major technical issues were identified. Several areas (e.g., liquid hydraulics) can be studied in scaled experiments at universities. Also, the existing $\mathcal{Z}$ facility may be used to study, e.g., radiation effects on wall materials or fireball reradiation effects for wall shielding.

Target fabrication, characterization, and injection were discussed at length. Target manufacturing requires extreme precision of manufacture, extreme reliability of delivery, and a manufacturing cost orders of magnitude lower than current ICF target performance. For indirect-drive IFE, target fabrication is the main issue; for direct-drive IFE, target survival during target injection is the main issue.

\section{IFE Metrics and Development Paths}

Metrics for the various stages of development were discussed. These include

Concept Exploration (CE), Proof of Principle (PoP), Performance Extension (PE), Fusion Energy Development (FED), and DEMO. The IFE Road Map, as shown in Fig.1, was discussed and debated. Note that the mainline IFE approaches - heavy ions, KrF, and DPSSL's - are all presently at the PoP level. $Z$ pinches, light ions, and the fast ignitor are all at the CE level. The main thrust for the next decade is to develop an attractive IRE approach to IFE. 


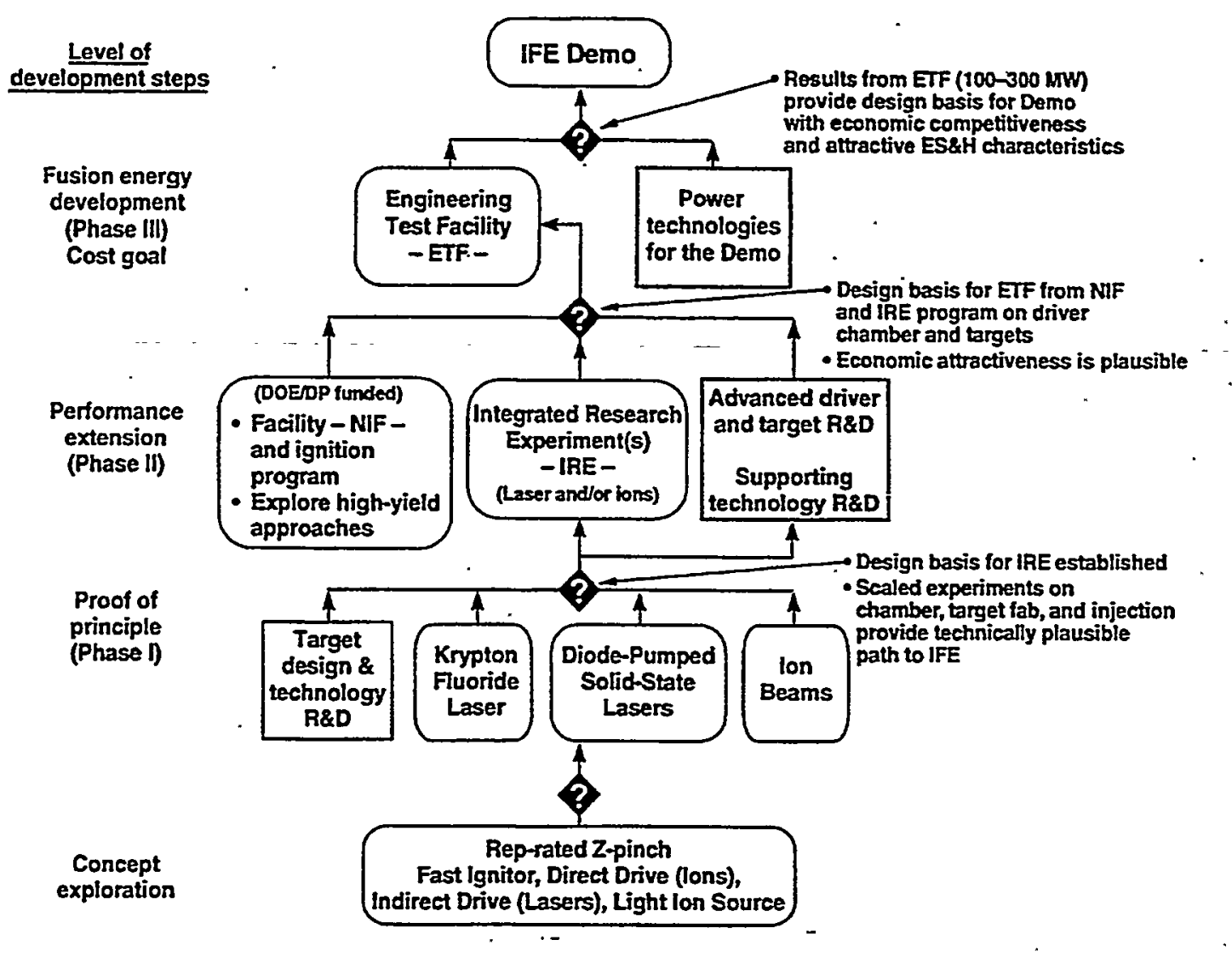

Fig. 1. The IFE Road Map

\section{Special Issues}

During the second week in IFE plenary sessions, the following list of special issues was discussed and debated. Very brief summaries of the results of these discussions are as follows:

(1) Why carry two laser options ( $\mathrm{KrF}$ and DPSSL)? The question as to whether to down select now to one or zero laser options was debated. The consensus was that more demonstrated results are needed to make a rational down selection. At present, the different strengths of the two laser approaches should justify continued research on both at this time.

(2) Should the IRE decision be delayed until there are results on NIF? Presently, an IRE decision would be made in about 5 years, the same time at which NIF will just be starting to get results. Wouldn't it be prudent to wait a couple of more years to see the initial NIF results? Presently, the IRE goal is to validate an integrated driver concept. An IRE will not implode capsules, and will typically be at only $\leq 1 / 10$ scale of a full driver. The purpose of the IRE is to investigate full system, rep-rate issues, in a scaled experiment; i.e., the IRE and NIF would be complementary. The IRE will help sort out target/driver/chamber choices. At Snowmass, it.was argued that there is sufficient confidence that NIF will work, and that the combined results of NIF + IRE would give the basis for moving on to the next stage (ETF). It was also argued that parallel 
development of target gain and driver/chamber technology (similar to parallel development of confinement schemes and fusion technology in MFE) is the most efficient.

(3) Is a demonstration of high yield/high gain needed for IFE (on a single-shot basis? This question was an extension of discussions that began in the Target group session. First, what is high yield? Present IFE power plant studies all have yields within the range of $300-800 \mathrm{MJ}$, at a rep-rate of 3-7 Hz. Therefore, the median choice for high yield is $500 \mathrm{MJ}$. The opposing view was to lower this requirement to, e.g.; $200 \mathrm{MJ}$ or less. This would, of course, increase the required rep-rate, and increase cavity-clearing difficulties. This issue was not resolved. To determine if a demonstration of high yield/high gain is needed, a good definition of the goals and cost of an ETF is needed. The ETF is after an IRE, and just before a DEMO. At the DEMO stage, there is, of course, agreement that high yield/high gain is required. Since all physics issues must be resolved before the DEMO stage, this means that the ETF, or before, should demonstrate high yield/high gain. During the first week of Snowmass, an ETF was defined to be a facility that is rep-rated at $\sim 25 \mathrm{MJ}$ yield, will demonstrate high yield in a separate chamber, with a driver energy of 1-2 $\mathrm{MJ}$, and at a cost of $<\$ 2 \mathrm{~B}$. When it was argued that the cost would more likely be $\$ 5 \mathrm{~B}$ for the listed requirements, the requirement for demonstration of high yield in the ETF was withdrawn (although this was not unanimous). The enduring theme is that the ETF should be rep-rated at $\sim 25 \mathrm{MJ}$ yield with a small-radius chamber to produce power-plant-level wall fluences. The issue of whether an ETF would demonstrate high yield in a separate chamber, and at what cost, was not resolved. During the IFE plenary discussions, the debate continued and there was a call for a vote. The question was "Do we need a single-shot, high-yield facility as a separate box on the road map?" The majority vote was a clear "no". However, the only place high yield is mentioned on the road map is under "explore high-yield approaches" inside the NIF box. It remains as an unanswered question as to where a high-yield demonstration will actually occur on the road map, which by definition, must be before a DEMO.

(4) Is the IFE program balanced between drivers, targets, chamber technology,

etc.? This question was in response to a request by Marshall Rosenbluth in his invited plenary talk on the first day of Snowmass to dedicate some portion of funds $(-\$ 10 \mathrm{M})$ to study beam propagation and chamber issues, and try to settle some of these questions once and for all. After initial discussions, a vote was called on the question "Should the relative amount of funding for chamber technology and transport \& focus be increased from current levels?" The vote was a unanimous "yes". This means that even within the existing funding levels, the relative amount of funding within a particular program for studying beam propagation/chamber issues should be increased.

(5) Is further work on Metrics needed? It was clear that there is a strong need to establish quantitative metrics for the IRE, the ETF, etc., and that these need to be applied to all driver candidates in a uniform manner. It was suggested that a "Tiger Team" be created to develop a set of IFE metrics.

(6) What if NIF does not reach ignition? The discussions on this issue began with the statement that there is "sufficient confidence" that NIF will reach ignition. If it doesn't, then it depends on exactly why. For example, laser/plasma interaction is a possible issue. There will be an extended campaign to demonstrate ignition, and many targets and laser beam configurations can be tried. The time window will be about 10 years for indirect drive, and about 5 years for direct drive. In addition, a contingency plan could be formulated.

(7) Is there sufficient interaction between targets, drivers, chambers, etc.? One of the advantages of IFE is that the targets, drivers, and chambers can all be studied and developed separately. However, optimizing any one system without consideration for the requirements of the other parts may result in conflicts. Optimization must be done for the 
overall power plant system. Interactions are improving in this area and the discussions at Snowmass made important contributions to these mutual interactions.

Following are Summaries from the four Subgroups.

Subgroup 1: Targets

(Max Tabak, Jill Dahlburg, Rick Olson)

\section{Targets Summary (Max Tabak)}

The target physics subgroup discussed the following three questions:- Question 1A: What are the key scientific issues for validating each target concept and how can they be resolved? Question 1B: How can existing (and new?) facilities be used to test each concept? Question 1C: i) What IFE target physics issues will not be resolved on NIF? ii) What is required to get to high yield?; and iii) What is the significance to IFE of experimentally demonstrating high yield / high gain ? During the discussions, the third question actually turned into a debate concerning the related question of whether or not a single-shot high-yield facility is necessary prior to the ETF.

For Question 1A, three inertial fusion concepts were discussed: indirect drive, direct drive and the emerging concept, fast ignition. Also discussed was the emerging concept, magnetized target fusion. Each concept was sketched, difficulties were described, and research programs were laid out. The established concepts, direct and indirect drive with lasers, have benefited from decades of study and have established through detailed comparisons between experiment and theory a first principles understanding of many of their critical scientific issues: materials properties, hydrodynamic stability and symmetry control. In other areas, such as electron transport or laser-plasma instabilities, there is an adequate empirical basis to proceed, but a complete first principles understanding may be absent. In the design of targets driven by $\mathrm{z}$ pinches or heavy ion beams much of this understanding carries over. Fast ignition using a short pulse laser beam, while promising significantly higher target gains and more relaxed implosion requirements than other inertial fusion concepts, has the greatest physics uncertainties, particularly in the areas of laser light and hot electron transport. Magnetized target fusion is a batch burn concept that uses dense walls to implode a preheated plasma and magnetic fields to reduce thermal conduction losses.

For Question 1B, the use of a number of existing or planned facilities to validate these concepts was discussed. Apart from doing physics experiments (Rayleigh-Taylor instability, material properties and others), the National Ignition Facility (NIF) will be the integration-validation test for direct and indirect drive and possibly for fast ignition. The Nike laser at the Naval Research Laboratory has the smoothest beams of any fusion laser. These smooth beams can be used to study the Rayleigh-Taylor instability in planar geometry. The Omega laser at the University of Rochester, a Nova scale laser, can be used to study direct-drive and indirect-drive implosions in NIF configurations, addressing a variety of physics issues. The Z-machine at Sandia National Laboratories can be used to study z-pinch implosion physics, NIF foot physics, radiation flow for z-pinch driven ignition schemes and even magnetized target fusion. Magnetized target fusion can also be studied at ATLAS, to be constructed by Los Alamos National Laboratory, and at ShivaStar at the Air Force Research Laboratory. Heavy ion accelerators like that at GSI in Germany and the proposed HIF IRE can study heavy ion deposition .physics and responses in heated plasmas. Fast Ignition can be studied on the Vulcan laser $(50 \mathrm{~J}, 50$ terawątt) at Rutherford-Appleton Laboratory, at Gekko (50J now-500 J) at ILE, Osaka as well as other facilities in the United States, Germany and France. An ignition demonstration would require tens of kilojoules of pulsed compressed light, together with a NIF-scale driver. A goal of the Fast Ignition effort is to justify this expenditure on the NIF. 
For Question 1C, the approach to energy was discussed. Although pursuing ignition on the NIF is a DOE DP funded activity, it is critical to the IFE mission. Ignition on the NIF will validate much implosion and thermonuclear burn physics, but a balanced program involving DOE OFES is still required in target physics. Energy conversion issues specific to other drivers like ion beams or $\mathrm{z}$ pinches can't be studied on the NIF. Fast Ignition research is currently outside the DP program. The mainline direct and indirect laser schemes to be tested on the NIF are inconsistent with liquid wall protection schemes. New target designs are needed. The current NIF laser indirect drive point design does not lead to adequate gain with affordable lasers.

Is a separate high-yield facility necessary before an Engineering Test Facility

(ETF) is built and what are possibilities for it? New designs may demonstrate high yield on the NIF as constituted or with some upgrade. A \$1-2 B z-pinch could achieve high yield, as might a heavy ion driver (but a cost was not discussed). Ultimately, we need to demonstrate high gain before the DEMO energy conversion unit is built, but $25 \mathrm{MJ}$ yields are adequate to test engineering features in an ETF.

Following are summaries of the three sessions on Targets.

\section{Question 1A: What are the key scientific issues for validating each target concept and how can they be resolved?}

Session Leader: Max Tabak

Speakers to initiate discussions:

$\begin{array}{ll}\text { Indirect Drive } & \text { John Lindl } \\ \text { Direct Drive } & \text { Jill Dahlburg } \\ \text { Fast Ignitor } & \text { Michael Key } \\ \text { Magnetized Targets } & \text { Irv Lindemuth } \\ \text { Other Concepts } & \text { Friedwardt Winterberg }\end{array}$

\section{Session Summary (Max Tabak)}

\section{Indirect Drive}

In indirect drive, the most studied inertial fusion scheme, energy from an outside source such as laser light, an ion beam or the kinetic energy in $\mathrm{z}$-pinch is converted into $\mathrm{x}$ rays. These $x$ rays then transport from the location where they are created to the implosion capsule where they are absorbed by the ablator. The reaction to the expansion of the heated ablator material then drives the capsule implosion. Ignition relies on the formation of a hotspot (with scale that of an alpha particle range) which accumulates much of the energy of the implosion. Burn then propagates to the high-density main fuel where the bulk of the yield is produced.

This scheme allows the separation of capsule implosion physics from driver coupling physics; so ignition on the NIF would validate implosion capsule designs for other drivers. In particular, thermonuclear burn physics, adiabat control through control of the temporal history of the radiation temperature, and hydrodynamic stability of the implosion would be validated on the NIF. Models of radiation transport can also be validated with laser facilities.

There are driver specific issues, however. For laser drivers, the laser-plasma interaction is a critical issue. Although this coupling physics is not completely understood, the work during the NOVA Technical Contract and more recently shows that laser-plasma instabilities can be adequately controlled through beam smoothing. Because projected laser efficiencies are typically $10 \%$ or less, target gain is a critical issue. The efficiency-gain product should exceed 8-10 for adequate cost of electricity. The current baseline indirect-drive laser targets have insufficient gain. However, preliminary 
calculations of new designs with improved coupling efficiency from hohlraum to capsule show that indirect drive with lasers should not be ruled out at this time.

Current heavy ion target designs, although sophisticated, have more than adequate gain in integrated ion-beam deposition/radiation-hydrodynamics/thermonuclear burn calculations (130 at $3 \mathrm{MJ}$ ) given driver efficiencies in the $25-40 \%$ range. Capsule stability is thought to be acceptable, based on single mode growth factor calculations. The growth factors calculated are less than or equal to those calculated for the NIF point design where very detailed 2D/3D multimode calculations have been performed. Similar calculations are required for the heavy ion designs. The detailed deposition profile for ion beams and the hydrodynamic response of the converter to the deposited energy affect the locations of radiation generation and hence the illumination symmetry on the capsule. However, by varying converter properties in the hohlraum the capsule design can accommodate various deposition profiles. There is an ongoing European program to measure and understand heavy ion stopping powers in hot matter. Also, preheat of the capsule may be caused by radiative relaxation of the atomic states of the in-flight ions or by the nuclear fragmentation of the incident ions. These effects are believed not to be critical based on measured nuclear fragmentation cross sections and calculated atomic relaxation rates: The sensitivity to beam pointing and power balance can be addressed with $2 \mathrm{D} / 3 \mathrm{D}$ radiation-hydrodynamic codes.

There are currently three schemes to drive capsules with $\mathrm{x}$ rays produced by $\mathrm{z}$ pinches. Two of these schemes involve producing radiation in primary hohlraums and then transporting the radiation to a secondary hohlraum that holds the implosion capsule. For these designs, the major issues are the coupling efficiency from the primary hohlraum to the implosion capsule and the symmetry and temporal history of the radiation driving the capsule. The third z-pinch design, the so-called dynamic hohlraum, involves imploding a z-pinch wire array upon foam that surrounds the implosion capsule. This design has much higher coupling efficiency than the prior two. The issues here are the stability of the wire array, its effect on the symmetry of the radiation field and the temporal history of the intensity of the radiation. These issues can be resolved by a combination of 2D/3D MHD calculations and experiments with $\mathrm{z}$ pinches.

Some target design issues involve interactions with reactor design and target fabrication requirements. For instance, the hohlraum materials chosen must be easy to recycle, minimize the induced radioactivity and have high opacity to $x$ rays. The target must also be inexpensive to fabricate. Green light is less stressing for laser design than blue light, but it is not a favored option for target design/plasma physics reasons. Can the new beam smoothing options coupled with lower intensity target designs make this an acceptable option? Current indirect-drive laser illumination schemes place beams over $2 \pi$ steradians. This is not consistent with current liquid first-wall power plant concepts. New target designs are needed to remedy this. On the other hand, heavy ion targets are limited only by the packing requirements of the final magnetic focussing elements in the reactor wall. Relaxing the beam spot requirement is a major challenge for heavy ion target design.

\section{Direct Drive}

The primary advantages of direct-drive laser fusion are (1) simplicity of the target, (2) excellent coupling efficiency (demonstrated to be greater than $80 \%$ ) because it avoids the energy conversion phase of indirect drive, and (3) like all IFE concepts, the driver is removed from the target chamber. It shares many critical issues with indirect drive: efficient coupling, keeping fuel on a low adiabat, demonstrating implosion symmetry, and demonstrating sufficient target stability. Although direct drive is normally done with lasers, there are proposed efficient designs that use ion beams to directly illuminate a capsule. However, the hydrodynamic stability properties of these designs are 
questionable. 2D/3D hydrodynamic calculations coupled with future experiments on an IRE would be required to settle the issue. For now, direct drive is primarily a laser concept.

Of the direct-drive issues the most stressing is hydrodynamic stability. Most schemes raise the ablator adiabat to increase ablation stabilization. A recent design uses radiation produced in an outer high-Z layer to engineer a tailored adiabat that is RT stable at the ablation front (high-adiabat) and yet maintains cold (low-adiabat) ignitionappropriate conditions in the inner fuel region. 1-D calculations for this design produce gain 130 (when zooming is used) at 1.3 MJ, raising the gain curve a factor of three from earlier designs. However, no integrated 2-D multimode calculations have been carried through to burn for this design. Current calculations also do not include the effects of magnetic fields. Ultimately some 3-D hydrodynamic calculations will be needed as well as planar and convergent Rayleigh-Taylor experiments.

Experiments are ongoing at the University of Rochester and NRL leading to hoped-for validation of NIF direct-drive designs. The current experiments will provide equation of stäte and opacity data, beam energy balance limits and Rayleigh-Taylor growth rates to benchmark the computer codes.

Direct drive may require somewhat better DT ice and ablator finishes than does indirect drive. There is an ongoing target fabrication effort to meet these requirements. Direct-drive target designs usually require power plant chamber perforations spread over $4 \pi$ steradians, so current direct-drive targets use dry-wall or wetted-wall power plant concepts. However, target designs that use less than $4 \pi$ steradians would be useful. In one recent publication, the cone angle for uniform illumination is less than 55 degrees. Individual laser beams must be aimed to 25-micron accuracy in order to achieve good symmetry without sacrificing coupling efficiency (Note: It is possible to relax the pointing accuracy by overfilling the beams. This does reduce coupling efficiency). If the final optics are placed at 25 meters to protect them from x-ray and neutron damage, this translates to a 1 microradian pointing requirement.

\section{Fast Ignitor}

Fast ignition relies on a somewhat different ignition scheme from conventional direct or indirect drive. Instead of igniting in a hotspot in the center of the fuel formed during the implosion, a fast-ignited capsule will be ignited by a short pulse laser on the capsule surface after the fuel has stagnated. The implosion can be accomplished with lasers, ion beams or $\mathrm{z}$ pinches. Because ignition occurs after implosion, hydrodynamic instabilities can't quench the burn. Because the Fast Ignitor's compressed state has lower density than that of the conventional implosion, symmetry requirements can be relaxed somewhat. The nominal gain curve for the Fast Ignitor is a factor 5-10 above the conventional gain curves. This higher gain can be traded for fuels with low tritium loading or smaller driver energy. The resistance to mix implies relaxed target fabrication requirements. It may also be possible to relax some of the cryogenic requirements. If it works, the integrated IFE story becomes stronger. Unfortunately, there is no integrated calculation or adequate validation experiment at this time.

The major issue for the Fast Ignitor is coupling the ignition energy from the short pulse laser to the high-density ignition region. This issue is comprised of three others: transport of laser light close to the assembled fuel, coupling of laser light to hot electrons (or possibly ions) at critical density and transport of hot electrons from the critical density to the ignition region. Assembling the compressed fuel ablatively creates an overdense plasma with scale dimension comparable to the initial capsule radius. Ponderomotively boring holes through a long plasma to conduct the Fast Ignitor beam may prove difficult. Non-spherical implosion designs may reduce the scale of the coronal plasma where the ignitor beam would enter and simplify this problem. These implosion calculations can 
reliably be performed with existing codes. PIC codes predict laser-electron coupling efficiencies between $40 \%$ and $90 \%$. Efficiencies above $40 \%$ have been measured in some experiments. Transport of the hot electrons between the critical surface and the ignition region has quite rich physics with many possible plasma instabilities. The forward currents are $10^{4}-10^{5}$ Alfven currents, so magnetic fields and current and charge neutralization should be important. Magnetic fields, depending on the simulation technique, between 10's and 100's of megagauss are predicted. This physics must be investigated with a research program that supports laboratory experiments together with a 3D parallel PIC code and with a hybrid code that can deal with high background electron densities. The experiments should study holeboring through overdense plasmas, electron transport through highly compressed matter and aspherical implosions.

Near term experiments can be carried out with a single Petawatt class laser beam coupled with a high-energy long-pulse beam for plasma formation. If positive conclusions follow from this concept exploration, an ignition demonstration at NIF can be envisaged. This would require converting up to $10 \%$ of the NIF beams to short pulse ignitor beams and using the remaining beams for fuel compression. A bottom line economic question that affects the technology is: how much ignitor energy will be required to make the concept robust?

\section{Magnetized Targets}

Magnetized target fusion is an emerging concept between magnetic confinement and inertial confinement. In this approach, a preheated plasma with an embedded magnetic field is squeezed by a liner implosion to ignition conditions. The magnetic field reduces the thermal conduction losses while the liner confines the plasma and supplies the energy required to heat it. The magnetic field allows lower implosion velocities and hence driver powers than IFE while the inertial confinement of the fuel allows lower stored magnetic field energy than MFE.

There are several issues. This is a batch burn concept. It was unclear how to refuel the target after ignition was obtained. Will the $Q$ be big enough for IFE? There have been no MHD calculations demonstrating a target with $Q$ large enough for an energy mission. The tools are available to do this calculation and it should be done. A small amount of high-Z pollutant injected into the plasma will radiatively cool the plasma and kill ignition. Are there designs that minimize mix and still have adequate $\mathrm{Q}$ ?

The development cost for this concept is low because there is little capital expenditure for experiments. The experiments can be conducted on existing facilities.

\section{Question 1B: How can existing (and new?) facilities be used to test each concept?}

Session Leader: Jill Dahlburg

Speakers to initiate discussions:

\section{NIF}

Omega

NIKE

Z

GSI

Liner Drivers

Petawatt (PW) laser for Fast Ignitor
John Lindl

Richard Town

Jill Dahlburg

Rick Olson

Max Tabak

Irv Lindemuth

Mike Key

\section{Session Summary (Jill Dahlburg)}

Following are summaries of the key points of each talk and associated issues. 
$\underline{\text { NIF }}$

KEY POINT: The NIF will enable integration experiments which include: gain energetics; pulse shape and compression; hydrodynamic stability and impact on direct drive; symmetry for indirect drive; and ignition and burn.

Q: What is the metric for success?

A: Target gain.

Information obtained from NIF experiments will carry over to:

(a) heavy ions (e.g., capsule physics, symmetry control, and hohlraum energetics);

(b) direct drive (e.g., hydrodynamic stability (imprint and target fabrication)); and

(c) maybe even be able to test the fast ignitor concept.

\section{OMEGA}

KEY POINT: The OMEGA laser facility at the University of Rochester's Laboratory of Laser Energetics addresses critical direct-drive and indirect-drive physics issues.

The OMEGA laser is a 60-beam glass laser system capable of delivering up to 30 $\mathrm{kJ}$ in a variety of pulse shapes of relevance to direct-drive and indirect-drive target designs. Achieving a high degree of illumination uniformity is crucial for the successful implosion of direct-drive IFE targets. Long-wavelength (low l-mode) perturbations are seeded by beam to beam variations. Short-wavelength (high $\mathrm{l}$-mode) variations are seeded by structure within individual beams. To control the high 1-mode structure, OMEGA uses 2D SSD. The current implementation of 2D SSD has a UV bandwidth of $0.3 \mathrm{THz}$ with 3 color cycles. In FY2000, the bandwidth will be increased to $1 \mathrm{THz}$ with 1 color cycle. A further reduction of sqrt(2) in the instantaneous non-uniformity will be achieved when polarization smoothing is also added in FY2000. Control of low 1-mode requires power balance and accurate beam pointing. OMEGA will demonstrate power balance of $3-4 \%$ in a 400 -psec window during FY2000 and routinely achieve pointing accuracy of 10 microns rms.

Although originally conceived as a direct-drive implosion facility, OMEGA has proven to be an extremely flexible system capable of performing direct-drive planar foil studies and indirect-drive hohlraum experiments. For example, the OMEGA laser was the first facility to be able to control the low-order mode asymmetries (P2 and P4) in indirect-drive capsules by means of beam phasing on multiple beam cones. In planar beam geometry, experiments have been performed to study laser imprint and the Rayleigh-Taylor (RT) instability. With the completion of a planar cryogenic handling system in 2001, OMEGA will be capable of extending its EOS studies and RT growth measurements to cryogenic deuterium.

By the end of 1999 , the cryogenic handling system for direct-drive implosion experiments will be installed. This facility will enable layering studies (using IR heating) of deuterium and DT ice in spherical capsules which are representative of IFE targets. During 2000, deuterium cryogenic implosion experiments will be undertaken with thin wall ( 1 micron) plastic shells. This will be followed by DT cryogenic experiments in 2001. These targets will be energy-scaled versions of those proposed for ignition experiments on the NIF. These experiments will integrate all aspects of the direct-drive IFE implosion design apart from ignition and burn propagation, and offer a severe test of the predictive design codes used for IFE target studies.

\section{NIKE}

KEY POINT: Nike is a flexible, uniform laser that is addressing outstanding physics issues that determine the success of a direct drive ICF pellet.

Nike is a $2-3 \mathrm{KJ}$ laser capable of the most uniform target illumination of all highenergy lasers suitable for fusion. The large bandwidths ( $3 \mathrm{THz}$ ) and advanced beam 
smoothing available with Nike allow better than 0.2 percent effective time-averaged illumination uniformity when overlapping 40 beams on planar targets. Nike experiments examine laser imprint, hydrodynamic instability, equations of state (EOS) for ICF materials, and other physics issues related to ICF. Nike provides the temporal laser pulse shapes needed to simulate the low isentrope compression and acceleration of pellet shells needed for high gain implosions. Peak intensities of up to $2 \times 10^{14} \mathrm{~W} / \mathrm{cm}^{2}$ are available for planar-target acceleration experiments. The planar geometry allows superior diagnostic access and allows acceleration of targets whose thickness approaches that of high gain target shells with modest laser energy. The Nike diagnostic suite includes high resolution, single line crystal $x$-ray imagers that are used to detect the lateral mass flow due to hydrodynamic instability in laser-accelerated targets.

Nike experiments are fielded to address the design criteria for high pellet gain, with particular emphasis on control of the target isentrope and inhibition of the RayleighTaylor instability. In initial work, very low levels of laser imprint, equivalent to better than 100 Angstroms surface finish, were inferred from plastic targets accelerated with the Nike uniform illumination. Recent work has concentrated on examining the underlying physics of advanced target designs where the ablator is preheated but the fuel remains cold. This work includes hydrodynamic instability measurements with x-ray preheated plastic and deuterium-loaded foam targets; EOS measurements of candidate materials including foams and deuterium loaded foams; and emission measurements of x-ray producing layers in targets that preheat the ablator and that may also ameliorate laser imprint.

$\underline{Z}$

KEY POINT: The large volume, long pulselength hohlraums together with an extensive suite of $x$-ray and shock diagnostics on $Z$ offer the opportunity to study a number of key physics issues relevant to indirect-drive ICF target concepts.

The $\mathrm{Z}$ pulsed-power facility is presently capable of providing hohlraum drives with pulselengths ranging from $\sim 3$ ns to $\sim 15$ ns in length and peak hohlraum $x$-ray input powers of up to $\sim 15 \mathrm{TW}$ in small $(\sim 6 \mathrm{~mm}$ diameter) hohlraums and $\sim 30 \mathrm{TW}$ in large $(\sim 17$ mm diameter) hohlraums. Hohlraum temperatures on $\mathrm{Z}$ range from $\sim 80 \mathrm{eV}$ in long-pulse, large hohlraums to $\sim 150 \mathrm{eV}$ in short-pulse, small hohlraums. The $\mathrm{Z}$ diagnostic suite includes XRD arrays, transmission-grating spectrometers, PCD arrays, soft X-ray framing cameras, laser velocity interferometry, and a laser active shock breakout system. A laser backlighter system will also be available on $\mathrm{Z}$ beginning in about 2001 .

The $-80-150 \mathrm{eV} x$-ray driven hohlraums on $\mathrm{Z}$ provide a platform for studying a number of the key physics issues of indirect-drive ICF targets. These include: hohlraum energetics, hohlraum wall opacity, wall motion in filled hohlraums, hohlraum hole closure, capsule ablator EOS, DT EOS, shock propagation in capsule ablator materials, and ablator burnthrough. In addition, a number of issues that are specific to z-pinch driven indirect-drive ICF concepts can also be studied with the $\mathrm{Z}$ facility. These include z-pinch implosion energetics and reproducibility, temporal shaping of the $\mathrm{x}$-ray drive, $\mathrm{x}$ ray transport and symmetrization, and capsule preheat.

\section{$\underline{\text { GSI }}$}

KEY POINT: GSI provides a capability to measure changes in ion packet energy as those packets are propagated through laser-produced plasmas. It can provide stopping power data relevant to heavy ion fusion.

The plasmas are produced with a $100 \mathrm{~J}$ on-site laser ( $1 \mathrm{~kJ}$ in the future), which heats foils. Ion beams are passed through the expanding plasma. Ion packet energy modification can be inferred from measured changes in ion packet arrival times. 


\section{Magnetized Target Fusion (MTF) Liner Facilities}

KEY POINT: Because of existing and near-term pulsed power facilities (e.g., ShivaStar at AFRL and Atlas at LANL), liner-driven MTF can be explored without any major capital investment.

Magnetized Target Fusion (MTF) was presented as an unexplored path to fusion that is a blend of MFE and IFE in the sense that the magnetization of the fuel reduces thermal losses (a la MFE) and the predominant heating mechanism is compressional heating by an imploding pusher (a la IFE). The optimal time and density scales for magnetized targets are intermediate between MFE and IFE. Operation at the intermediate density allows MTF systems to be much smaller than MFE systems and to require much lower power and intensity from the drive than IFE systems. Although any implosion driver can be considered as an implosion driver candidate for magnetized targets, MTF advocates have chosen magnetically-driven liners as the lowest cost path to evaluating the principles of MTF and demonstrating a burning plasma.

A recent OFES-funded liner experiment on ShivaStar demonstrated liner performance suitable for significantly compressing a field-reversed configuration. The 30 $\mathrm{cm}$ long liner had an initial radius of $5 \mathrm{~cm}$. Radiographs and other diagnostics indicated that the liner achieved a kinetic energy of $1.4 \mathrm{MJ}$, with excellent symmetry at a radial convergence of 12 . The Atlas facility, operational in 2001, will be able to drive MTF liners to more than $10 \mathrm{MJ}$ at MTF-relevant velocities.

\section{Addition to NIF (Petawatt laser) for Fast Ignitor}

KEY POINT: Most of the laser technology needed to convert NIF beams to fast igniter beams has been demonstrated in the Petawatt beamline at NOVA. A full-scale high-gain fast ignition demonstration at NIF could be carried out if about $10 \%$ of the NIF beams were used for ignition and the rest for fuel compression.

Prior to any full-scale test, basic science studies are needed to establish the feasibility of transporting energy efficiently from igniter laser beams to the ignition spark. Such experiments can be carried out with a single Petawatt beam line coupled with a high-energy plasma-forming beam line. This is technically possible either at NIF or in a separate facility such as SPIRE (a continuation of the Nova-Petawatt program).

Transmission grating compressors are needed for the igniter beams to deliver more energy in longer pulses and this technology is currently being developed along with other diffractive optics for NIF. Current technologies for beam quality by adaptive optics and prepulse control are adequate for fast ignition.

\section{Question 1C: (i) What IFE target physics issues will not be resolved on NIF? \\ (ii) What is required to get to high yield? \\ (iii) What is the significance to IFE of experimentally demonstrating high yield/high gain?}

Session Leader: Rick Olson

Speakers to initiate discussions:

Indirect-drive target issues not resolved on NIF

Direct-drive target issues not resolved on NIF

What's required to get to high yield (ZX, X-1)

Max Tabak

Richard Town

What's required to get to high yield (NIF Upgrade) Mark Herrmann

High-yield/high-gain demonstration not needed John Lindl 


\section{Session Súmmary (Rick Olson)}

Max Tabak and Richard Town léd discussions concerning the target physics issues that would not be addressed on NIF. Max initiated discussions on four indirect drive issues that either cannot (because it's not possible) or will not (because they're not in the plan) be investigated on NIF. The indirect-drive issues are: 1) two-sided illumination where a small solid angle is subtended by laser beams; 2) laser illumination with green light (there was some disagreement on this); 3) physics issues specific to other drivers (e.g., ion deposition); and 4) indirect-drive implosion coupled to fast ignition. Richard led the discussions concerning direct-drive target issues that would not be resolved on NIF. He began by stating that " $90 \%$ of direct-drive issues can be examined on NIF". The remaining $10 \%$ that cannot be resolved on NIF include: 1) high-yield implosions; 2) high rep rate and target deployment suitable for energy application; and 3) target chamber and final optics material issues. Although there was much group discussion concerning past and potential future experiments on Omega, NIKE, and NIF, the overall conclusions concerning what could not be done in either pre-NIF or NIF experiments remained unchanged from the basic points originally. listed by Max and Richard.

Rick Olson, John Lindl, and Mark Hermann led the discussions concerning the second question -- What is required to get to high yield on either the $\mathrm{ZX} / \mathrm{X} 1$ or the NIF/NIF-upgrade paths? Rick believes that the $\mathrm{ZX} / \mathrm{X} 1$ path to high yield would require a combination of a $\sim 10$ year $R \& D$ program and a $\sim \$ 1 B$ facility. The $R \& D$ program would involve experiments (on the existing $\mathrm{Z}$ and an intermediate facility called $\mathrm{ZX}$ ) in the areas of pulsed power, hohlraum energetics, pulse shaping, and symmetry. For IFE, research in the areas of rep-rate, standoff, and reactor chamber concepts would also be required. To indicate the level of extrapolation that the $\mathrm{ZX} / \mathrm{X} 1$ path would involve, Rick made the comparison that, in terms of $\mathrm{A}_{\mathrm{w}} \mathrm{T}^{4}$ (which is proportional to power into a hohlraum), $\mathrm{Z}$ is similar to Nova in input power, but also has more energy (longer pulselength). There seemed to be agreement (on the part of SNL and LLNL) that the $\mathrm{ZX} / \mathrm{X} 1$ path is feasible but not assured. It was pointed out during Rick's presentation that LLNL has also calculated yields of $\sim 400-1000$ MJ using the X1 power pulse as input. John Lindl described a potential technique for high-yield target experiments on NIF. This involves a recently developed "advanced-coupling target" employing lower laser power, longer pulse shapes, new wall material mixtures, and larger capsule/hohlraum size with improved coupling efficiency. John described a recent unoptimized, 2D integrated calculation done by Larry Suter indicating $\sim 600 \mathrm{~kJ}$ absorbed in a capsule with a $\sim 70 \mathrm{MJ}$ yield. John indicated that straightforward improvements upon Larry's calculation will increase the calculated yield to $\sim 150 \mathrm{MJ}$. His "guess" for an upper limit on high yield calculations utilizing NIF laser input is $\sim 400 \mathrm{MJ}$. This is preliminary work in progress. John stated that high yield in NIF is "by no means assured". An item worth noting about the related discussions involves the question of whether or not a new NIF target chamber $(\sim 100 \mathrm{M})$ would be required for high-yield experiments. Apparently, it is possible that a limited number of high-yield experiments could be done in the existing NIF chamber. In a more general approach to the topic of "What's required for high yield?" Mark Hermann gave a presentation on results from capsule calculations ranging from $20 \mathrm{MJ}$ to $>$ GJ with the peak drive temperature chosen for $40 \%$ ignition margin. His basic conclusion is that bigger is easier - lower peak drive temperature is required for the same margin or, if we use a big capsule with $300 \mathrm{eV}$, it can be very robust.

The third question in this session turned into a debate concerning whether or not a single-shot high-yield facility is necessary and/or useful prior to the ETF. John Lindl stated that no new high-yield/high-gain facility is required prior to the ETF for either target physics or the development path reasons. Keith Matzen maintained that highyield/high-gain in a single-shot facility is needed for IFE and should be positioned on the 
roadmap at a time prior to the ETF. John's main points can be summarized as follows: 1) ignition and burn propagation physics is scale-size invariant; 2) differences between ion and laser targets can be tested in nonfusion experiments; 3) ETF requires $\sim 25 \mathrm{MJ}$ capsules (hence does not require high-yield capsules); 4) multiple chambers could allow high-yield tests in the ETF; and 5) high yield might be possible on NIF (as discussed previously). Keith presented a different point of view. He made the following points: 1) the DOE/DP has a high-yield mission need and might provide a $\sim$ \$B single-shot highyield facility; 2) the step from NIF to ETF is "enormous"; 3 ) the high-yield step adds value for IFE target design; 4) the high-yield step adds value for IFE chamber design; 5) the high-yield step reduces risk for ETF; and 6) the incremental cost to DOE/OFES would be small if DOE/DP funds the facility. There was a significant amount of discussion of the high-yield question throughout the entire session (even before John and Keith began their discussions), indicating a range of viewpoints on the issue. Jill Dahlburg and Rick Olson have a rather extensive set of notes from the session, indicating some of the various positions taken by the attendees (who represented nine different research institutions).

\section{Subgroup 2: Drivers and Standoff \\ (Steve Payne, John Sethian, John Barnard, Rick Spielman)}

\section{Drivers and Standoff Summary (Steve Payne)}

The Drivers and Standoff Subgroup discussed questions relating to (1) individual driver characteristics, (2) standoff issues, (3) and what is required for a convincing IRE scenario for each driver candidate. Throughout these discussions, five driver candidates were considered. As mentioned earlier, the mainline.IFE driver candidates (which are at the PoP level) are heavy ions, $\mathrm{KrF}$ lasers, and DPSSL's; the other candidates (which are at the CE level) are $\mathrm{z}$ pinches and light ions. MTF was explicitly discussed, but not considered to be directly in the IFE category.

The five driver candidates are briefly illustrated in Table 4, and their key characteristics are listed in Table 5. The U.S. heavy ion driver is an induction linear accelerator that creates a high current (10's to 100's of $\mathrm{kA}$ ) beam of multi-GeV heavy ions. The $\mathrm{KrF}$ laser uses electron-beam pumping of a gas mixture to produce a UV laser beam $(248 \mathrm{~nm})$, whereas the DPSSL uses diode arrays to pump a solid-state medium to produce an IR laser beam that is frequency-tripled into the UV (350 nm), analogous to NIF technology. The z-pinch uses a very high-current pulsed-power driver (20 MA on Z) to pinch a wire array on axis to make an intense $\mathrm{x}$-ray source. A light-ion driver uses a high-current light-ion diode to produce a high-current ( MA) ion beam at moderate energy (10's of MeV). A summary of current values of parameters (brightness, uniformity, pulse shaping, efficiency, durability, rep-rate, cost) is given in Table 5.

Standoff issues refer to interface issues between the driver and the target, as summarized in Table 6 . For heavy ions, the mainline approach uses ballistic transport with a quadrupole magnet array for final focus, with a standoff distance of the order of many meters. Other options include neutralized transport, and channel transport. For the UV lasers, the final optic may be a grazing incidence metal mirror ( $\mathrm{KrF}$ or DPPSL), or a hot fused silica wedge or grating (DPPSL). For a z-pinch, a recyclable transmission line (RTL) concept is being considered. For light ions, transport may be neutralized ballistic transport with a lens system, or any of several forms of channel transport. Every standoff scenario needs further study.

Results of the IRE discussions are roughly summarized in Table 7. In this Table, the weakest link is the " $E$ " category, which requires further physics/engineering exploration. In particular, note that the key issues are neutralized and channel transport 
for $\mathrm{HIF}$, durability for $\mathrm{KrF}$ (survivability of pumping foil), cost of diodes (DPPSL), durability for $\mathrm{z}$ pinches (ability to recycle the RTL), and durability (ion source performance and reliability) and final transport for light ions.

An overall driver summary is given in Table 8 . The results were agreed upon only after extensive discussions (!). In each case, the bar shown was quantized to only three levels (small, medium, or large). Very roughly, this Table shows the various strengths and weaknesses of the various drivers: Our IFE development plan, current research approaches, 'and IRE development studies will sort out the potential of each driver to meet the requirements for a successful IFE system.

Following are summaries of the three sessions on Drivers and Standoff.

Table 4. Five driver options are being considered for IFE.

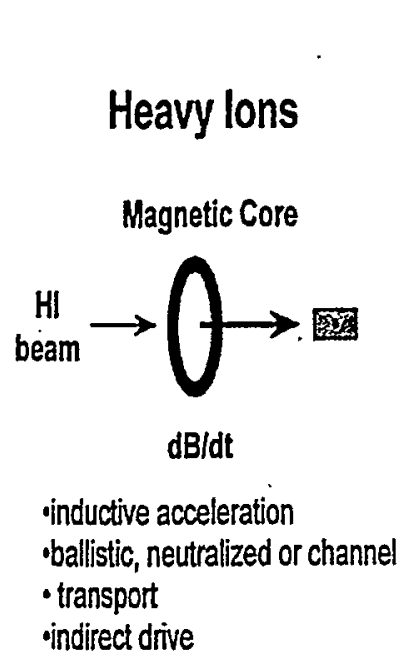

Proof of Principle
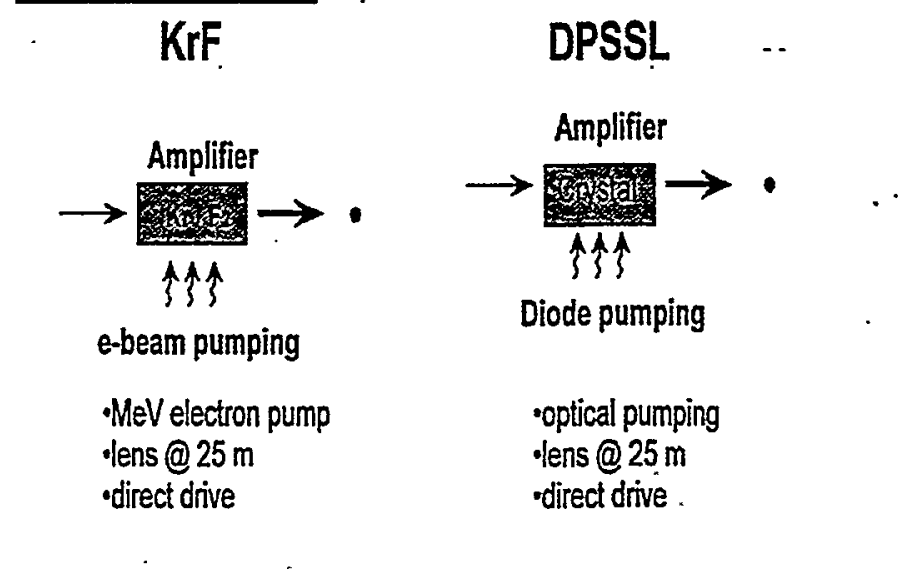

Concept Exploration

\section{Z-Pinch}

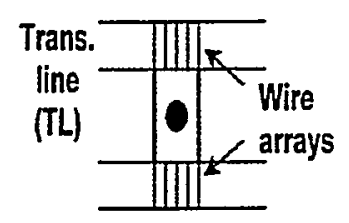

-60 MA \& 8 MV drive replaceable TL indirect drive
Light lons

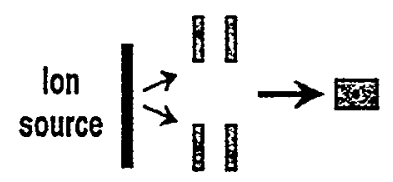

-HV acceleration

- neutralized or channel transport indirect drive

\section{Other Possibilities}

- MTF

- Medium weight

ion accelerators

Table 5. Status and potential of IFE drivers.

\begin{tabular}{|l|l|l|l|l|}
\hline Drivers & Brightness & Uniformity & Shaping & Efficiency \\
\hline Heavy Ions & $\begin{array}{l}\text { Ion source } \\
\text { brightness suitable }\end{array}$ & $\begin{array}{l}\text { X-ray } \\
\text { smoothing }\end{array}$ & $\begin{array}{l}\text { Velocity tilts and/or } \\
\text { separate beams for } \\
\text { foot and main pulses }\end{array}$ & $\begin{array}{l}45 \% \text { for Kr, 32\% for Pb; } \\
\text { deduced from e-linacs and } \\
\text { core loss measurements }\end{array}$ \\
\hline
\end{tabular}




\begin{tabular}{|l|l|l|l|l|}
\hline KrF & $\begin{array}{l}3 \times 10^{1 /} \mathrm{W} / \mathrm{cm}^{2} \text { str.; } \\
10 \times \text { requirement }\end{array}$ & $\begin{array}{l}0.2 \% \text { meas. for } \\
\text { beam; meets } \\
\text { spec. }\end{array}$ & $\begin{array}{l}\text { Pulse stacking; } \\
\text { meets spec. }\end{array}$ & $\begin{array}{l}7 \% \text { by component } \\
\text { validation }\end{array}$ \\
\hline DPSSL & $\begin{array}{l}3 \times 10^{18} \mathrm{~W} / \mathrm{cm}^{2} \text { str.; } \\
100 \mathrm{x} \text { requirement }\end{array}$ & $\begin{array}{l}0.04 \% \text { calc.; } \\
0.2 \% \text { needed on } \\
\text { target }\end{array}$ & $\begin{array}{l}10^{4}: 1 \text { demonstrated; } \\
\text { meets spec. }\end{array}$ & $\begin{array}{l}10 \% \text { by component } \\
\text { validation }\end{array}$ \\
\hline Z-pinch & $\begin{array}{l}\text { x rays from wire } \\
\text { array drive hohlraum }\end{array}$ & $\begin{array}{l}\% \text {-level } \\
\text { demonstrated }\end{array}$ & $\begin{array}{l}\% \text {-level } \\
\text { demonstrated }\end{array}$ & $\begin{array}{l}15 \% \text { (wall plug to } \mathrm{x} \text { rays) } \\
\text { demonstrated }\end{array}$ \\
\hline Light Ions & $\begin{array}{l}10 \% \text { of IFE; } \\
\text { second-stage diode } \\
\text { needed }\end{array}$ & $\begin{array}{l}\text { x-ray } \\
\text { smoothing }\end{array}$ & $\begin{array}{l}\text { Velocity tilts and/or } \\
\text { separate beams for } \\
\text { foot and main pulses }\end{array}$ & $64 \%$ demonstrated \\
\hline
\end{tabular}

Table 5. Continued.

\begin{tabular}{|l|l|l|l|}
\hline Drivers & Durability & Rep-rate & Cost \\
\hline Heavy ion & $\begin{array}{l}10^{8} \text { shots; based on } \\
\text { Astron and improved } \\
\text { source }\end{array}$ & $10 \mathrm{~Hz}$ & $\begin{array}{l}\$ 150 / \mathrm{J} \text { for } \mathrm{Kr} ; \\
\$ 230 / \mathrm{J} \text { for Pb; } \\
\text { vendor estimated }\end{array}$ \\
\hline KrF & $\begin{array}{l}10^{2} \text { shots currently; } \\
10^{5} \text { shots in Phase } 1\end{array}$ & $\begin{array}{l}100 \mathrm{~Hz} \text { in literature } \\
\text { demonstrated }\end{array}$ & $\begin{array}{l}\$ 225 / \mathrm{J} ; \\
\text { extrapolated costs }\end{array}$ \\
\hline DPSSL & $\begin{array}{l}10 \mathrm{~Hz} \text { in small test } \\
\text { bed; meets spec. }\end{array}$ & $\begin{array}{l}\text { \$400/J; assumes } 5 \mathrm{c} / \mathrm{W} \\
\text { diodes, large extrapolation }\end{array}$ \\
\hline Z-pinch & $\begin{array}{l}\text { Single shot now; } 10^{2} \\
\text { shot burst mode with } \\
\text { replaceable trans. line } \\
\text { for } \mathrm{RE}\end{array}$ & $\begin{array}{l}\text { Single shot now; } \\
0.1 \text { Hz with } \\
\text { replaceable trans. } \\
\text { line }\end{array}$ & $\begin{array}{l}\$ 30 / \mathrm{J} \text { of x rays } \\
\text { demonstrated }\end{array}$ \\
\hline Light ion & $\begin{array}{l}10^{4} \text { shots; many issues } \\
\text { to resolve }\end{array}$ & $\begin{array}{l}\text { Single shot now; } \\
\text { ultra-pure carbon } \\
\text { anode needed }\end{array}$ & $\$ 150 / \mathrm{J}$ demonstrated \\
\hline
\end{tabular}

Table 6. Standoff issues and how they are being addressed. 


\begin{tabular}{|c|c|c|}
\hline Drivers & $\begin{array}{l}\text { Final Oplic or } \\
\text { Power Feed Lifetime }\end{array}$ & $\begin{array}{l}\text { Power Transport Efficiency; } \\
\text { Focisabilithy }\end{array}$ \\
\hline Heaw lons & $\begin{array}{l}\text { Design superconducting final } \\
\text { optic based on dala and *- } \\
\text { neutronics. }\end{array}$ & $\begin{array}{l}\text { Assess neutralization and } \\
\text { channeling }\end{array}$ \\
\hline Kif & \multirow[t]{2}{*}{$\begin{array}{l}\text { Metal mirror and heated silica } \\
\text { studied at low-lo-moderate dose } \\
\text { studied }\end{array}$} & $\begin{array}{l}100 \% \text { transport al }<0.5 \text { Tor of } \mathrm{Kr} \\
\text { to reduce } x \text {-ays }\end{array}$ \\
\hline DPSSL & & $\begin{array}{l}\text { Assess gas-breakdown and } \\
\text { target heating issues }\end{array}$ \\
\hline Z-Pinch & $\begin{array}{l}\text { Develop replaceable tansmission } \\
\text { line }\end{array}$ & $\begin{array}{l}\text { 67\% transport through present TL } \\
\text { X-Fays trom wire array dive } \\
\text { hohhraum }\end{array}$ \\
\hline $\begin{array}{l}\text { Light } \\
\text { lons }\end{array}$ & Assess/manage irradiation of lens & $\begin{array}{l}\text { Assess neutralization and } \\
\text { channeling }\end{array}$ \\
\hline
\end{tabular}


Table 7. Status of IRE concepts.

(Key: $\mathbf{I}=$ integrate, $\mathrm{V}=$ validate, $\mathrm{E}=$ explore)

\begin{tabular}{|c|c|c|c|c|}
\hline Drivers & $\begin{array}{l}\text { Brighiness } \\
\text { and } \\
\text { Uniformily }\end{array}$ & $\begin{array}{c}\text { Focusability \& } \\
\text { Chamber Transport }\end{array}$ & Durability & $\begin{array}{l}\text { Driver } \\
\text { Cost }\end{array}$ \\
\hline $\begin{array}{l}\text { Heavy } \\
\text { lons }\end{array}$ & $\bar{V}$ & $\begin{array}{c}\text { V (ballistic transport); } \\
\text { E (neutralized \& } \\
\text { channeling) }\end{array}$ & $T$ & $T$ \\
\hline KiF & $T$ & $T$ & $\bar{E}$ & $T$ \\
\hline DPSSL & $T$ & $T$ & $T$ & $E$ \\
\hline 2-Pinch & V & 1 & $E$ & 1 \\
\hline Light lons & V & $\begin{array}{l}V \text { (neutralized); } \\
E \text { (channeling) }\end{array}$ & $E$ & 1 \\
\hline
\end{tabular}


Table 8. Driver options for IFE have a variety of strengths and weaknesses.

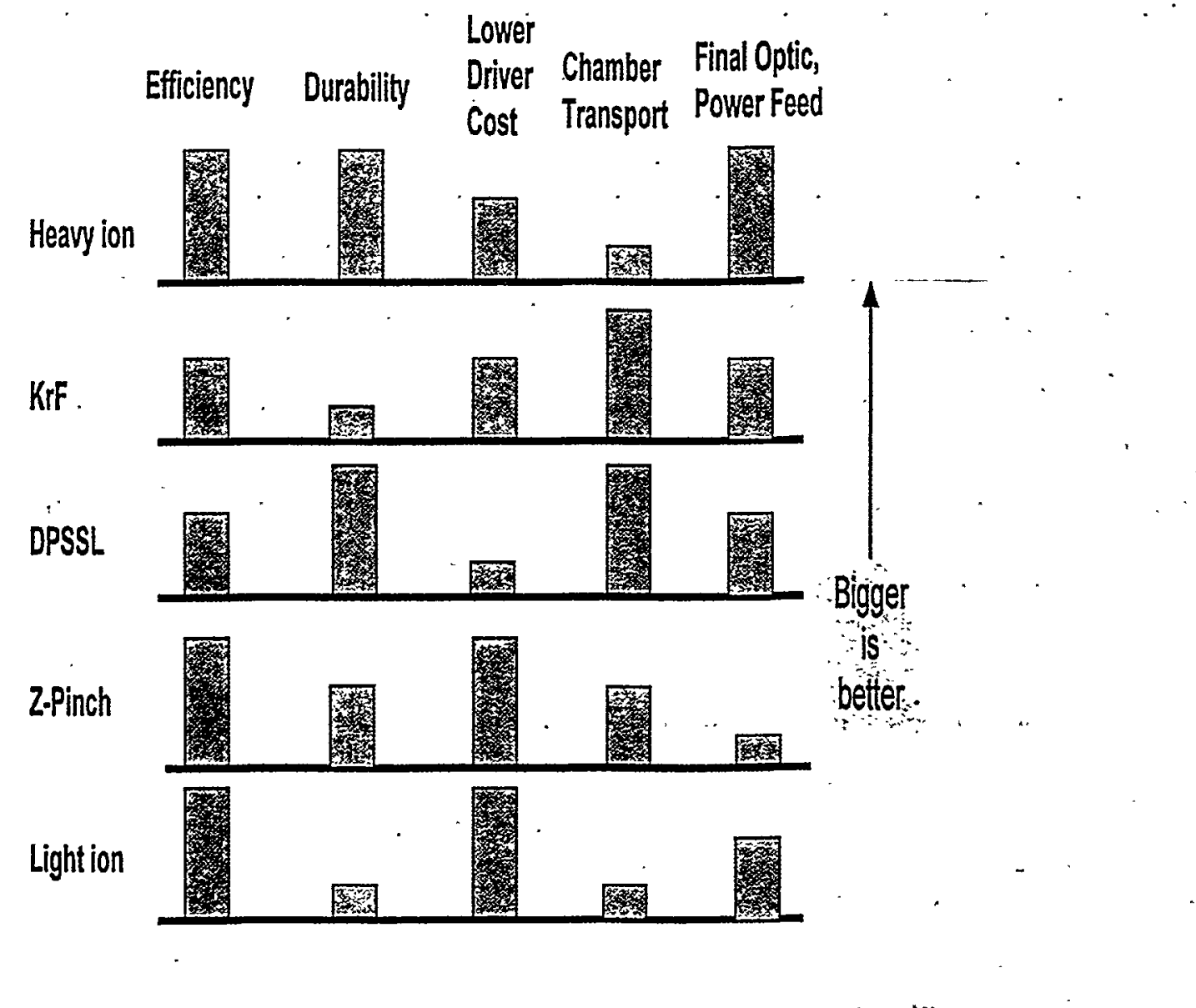

Question 2A: How can the source brightness, beam uniformity, pulse shaping accuracy, efficiency, reliability, repetition rate, and cost of each driver concept be improved?

Session Leader: John Sethian

Speakers to initiate discussions:

$\begin{array}{ll}\text { HIF } & \text { John Barnard } \\ \text { LIF } & \text { Mike Cuneo } \\ \text { KrF } & \text { John Sethian } \\ \text { Z-pinch } & \text { Rick Spielman } \\ \text { Other Concepts } & \text { Koichi Kasaya }\end{array}$

\section{Session Summary (John Sethian)}

We heard five excellent talks discussing the main driver concepts for IFE: heavy ions, light ions, $\mathrm{KrF}$ lasers, Diode Pumped Solid State Lasers (DPPSL's), and the z-pinch. All addressed the posed questions to one degree or another. We also had one auxiliary equally fine talk on Medium Ion Sources. The discussions were lively, probing and generally polite. A general consensus was impossible to reach, primarily because each advocate steadfastly (and properly) believes his or her driver is the most promising approach. However a few points were agreed upon: 
All of the candidate drivers have promise. All of them have distinct advantages and distinct challenges. Of the five, the heavy ions and two lasers are the most advanced. They have well-defined program plans, with clear cut metrics and goals that must be met for IFE. The z-pinch is a copious source of $x$ rays, but is in the concept exploration phase with respect to inertial fusion energy, and faces challenging issues that must be addressed conceptually before a program can be formulated. The light ion work is presently on hold due to problems with the source, but a new type of source may make these a viable candidate. Nevertheless, given the relatively immaturity of even the most developed of these concepts it seems inappropriate to totally exclude any of them.

A synopsis of each driver talk, and the related discussion, are reported below. We caution, however, in taking this as the sole basis for ranking one driver over another. In fact, the drivers need to be explored in the context of a complete Inertial Fusion Energy system. This requires a broad based science and technology program, with the explicit goal of validating critical technologies. The program must look at all the issues for each driver, the target gain, chamber, target fabrication, target injection, and ultimately, the cost of electricity.

\section{Heavy Ions}

Three different examples of heavy ion drivers were given, based on "bottoms up" systems codes. (A preferred configuration for a heavy ion accelerator - ion species, charge state, voltage and total energy, has not yet been chosen.) In a highlighted case, the driver energy is $6 \mathrm{MJ}$, uses $4 \mathrm{GeV}, 200 \mathrm{amu}(+1$ charge state) ions, and has a peak ion current of $4.1 \mathrm{kA}$ in each of 32 main pulse beams out of 48 beams. Heavy ion driver costs are projected to be in the range $\$ 0.64$ to $\$ 1.3 \mathrm{~B}$ (about $\$ 200 / \mathrm{Joule}$ ). This is based on systems studies, vendor estimates based on anticipated technology advances, and economies of scale. The typical cost reductions required for the different components, from the present technology to an IFE system, is within an order of magnitude. The exception is the superconducting magnet arrays, whose cost reduction is more uncertain because they are still in the design phase. The estimated costs of the magnet arrays, however, do reflect the current price of low-temperature superconductors and are based on experience with costs of actual accelerator superconducting magnets. The cost of a heavy ion accelerator can be improved by reducing the target energy requirements, reducing the length of the accelerator, or reducing the cost per unit length. The latter can be done by improving the unit costs or decreasing the diameter of the arrays and cores. Many programs are underway with vendors to reduce the cost further.

The efficiency of a heavy ion driver is anticipated to be $30-45 \%$. This is based on systems analysis of induction accelerators, which include a variety of energy loss mechanisms. The pulsed power for these machines was estimated to be $75 \%$ efficient, and core losses were based on measurements of core samples and actual cores driving resistive loads. A model of beam dynamics and transport was also incorporated into the systems code. The high efficiency of ion drivers is realized with several existing electron beam induction accelerators (for example, ATA was quoted to have an efficiency of "tens of percent"). Existing heavy ion induction accelerators have low efficiencies (about a few percent) because they are low current; however, as the current increases, the power driving a full-scale core does not increase substantially, so the accelerator efficiency is consequently expected to increase.

The beam brightness can be considered a measure of the phase space density, and for heavy ions the phase space density at the source (achieved in scaled experiments) should be 1000 times larger than what is required at the target. So the beam brightness should be adequate, but the flux capabilities are very low. Ion source research and development will be necessary to meet the flux and lifetime requirements. For example, the energy flux of the plasma lens experiment at GSI (with an $\mathrm{Ar}^{18+}$ beam) is $\sim 2 \times 10^{10}$ W/cm $\mathrm{cm}^{2}$, about a factor of 1000 lower than the $7 \times 10^{13} \mathrm{~W} / \mathrm{cm}^{2}$ needed per beam in one HIF 
design. On the other hand, the collider at RHIC will reach a peak energy flux of $7 \times 10^{14}$

$\mathrm{W} / \mathrm{cm}^{2}$ and the SLC electron-positron collider has a peak flux of $10^{21} \mathrm{~W} / \mathrm{cm}^{2}$, so some aspects of accelerator technology have already reached driver scale. The new requirement is high current along with high energy.

Requirements for the beam uniformity or pulse shaping of a heavy ion driver have not been determined, but are not expected to be an issue due to radiation smoothing. Beam centroid errors may be more stringent but still can be met; 2D target simulations allow $+/-200 \mu \mathrm{m}$ pointing errors, and preliminary tracking experiments indicate these are achievable.

Pulse-shaping requirements have also have not been finalized, but they are also not expected to be a problem. Two methods are proposed to achieve the proper foot/main pulse shape; using separate sets of beams for the foot and main pulse, or using velocity tilts to vary the energy. Optimally, a combination of both methods will probably be used. More simulations to verify these approaches need to be carried out.

Heavy Ion accelerators should meet the required rep-rate and lifetime, based on experience with electron linacs. It was pointed out that research accelerators such as LANSCE have availability exceeding $85 \%$ during scheduled operating runs.

\section{Light Ions}

Presently, light ion fusion development is on hold due to the lack of a sufficient ion source. Nevertheless, when the work was halted, the work had progressed to the point that only " 3 factors of 3 " were needed in parameter improvement for light ions to meet the anticipated requirements for a high-yield driver. Specifically, an ion energy of 12 $\mathrm{MeV}$ was reached, with $30 \mathrm{MeV}$ needed; current densities of $0.5 \mathrm{kA} / \mathrm{cm}^{2}$ were reached, with $1-2 \mathrm{kA} . \mathrm{cm}^{2}$ needed, and a $15 \mathrm{~ns}$ long pulse was achieved, with 40 ns needed. These three parameters were achieved simultaneously on PBFA $\Pi$. It is also necessary to decrease the ion beam divergence; 22 milliradians was achieved in single-stage diode, meeting the transverse particle energy requirement for fusion. A total divergence of 6-12 milliradians is needed; it is proposed to achieve this range in a post acceleration step at constant emittance. Subsequent work on the SABRE accelerator has demonstrated current densities that scale to fusion requirements at the necessary pulselength of $40 \mathrm{~ns}$. When the light ion program was placed on hold, beam production on SABRE had progressed to within a factor of 2 to 4 of injector stage requirements, without an optimal source.

The main stumbling block to achieving a viable light ion driver is the realization of a robust, pure, ion source with the required brightness. Past efforts concentrated on lithium ions, which proved unsuitable primarily because of impurity issues. The most promising candidate is now believed to be a $50 \mathrm{MeV}$ Carbon ion source. One reason for this is that the main impurity in the previous ion sources were carbon ions anyway. An $80 \%$ pure carbon source was also demonstrated on SABRE.

Other issues regarding the driver (pulse shaping, efficiency, reliability, rep-rate and cost) have not been fully considered.because of the fundamental question of a suitable source. Pulse-shaping would be accomplished with velocity tilts and/or separate beams for the foot and main pulses, as in HIF. A $60-70 \%$ ion production efficiency has been demonstrated in many previous experiments. For an initial demonstration, it is estimated that the source should have a lifetime of $10^{6}$ shots $(2$ weeks at $1 \mathrm{~Hz})$, and that the cost of a rep-rate machine about the size of Hermes III (the potential driver technology - albeit single shot) is about $\$ 80 \mathrm{M}$ for one (of 12) modules.

\section{Diode-Pumped Solid State Lasers (DPPSL)}

The Mercury Laser (100 J/10Hz/10\% efficiency) under development at LLNL will be a test bed to develop DPPSLs for fusion energy. DPPSL's have already meet the repetition rate required for IFE (up to $25 \mathrm{~Hz}$ in small apertures), and they should meet the 
durability requirements- current technology with DPPSL's show laser diode pump bars at about $100 \mathrm{~W} / \mathrm{cm} @ 10^{8}$ pulses, with $200 \mathrm{~W} / \mathrm{cm} @ 10^{10}$ pulses required. It is believed that advanced development of the pump diodes and optics will achieve the necessary lifetime, and a viable development path to achieve these was presented. The efficiency of DPPSL is expected to be $10 \%$ and could be as high as $20 \%$. These are "stem-to-stern" efficiency estimates, based on all the components in the system and include cooling and beam transport to target. The present projected aggregate efficiency of all the components, tested individually, is $9 \%$, assuming shaped pulses but excluding cooling or beam transport. The $10 \%$ figure represents the baseline goal for Mercury. The $20 \%$ figure assumes more advanced development.

The cost of a DPPSL is driven by the cost of the pump diodes. In Mercury (year 2001 ) the cost of the diodes is expected to be $\$ 3.00 /$ peak Watt. That number is needed to drop to $\$ 0.50$ /peak Watt for the IRE (2010), and to $\$ 0.05 /$ peak watt for an IFE system (2030). The estimates for cost reduction are based on systems studies of diode plant manufacturing, anticipated technology advances, and economies of scale. The diode manufacturing facility is assumed to produce one fusion power plant worth of diodes per year. As DPSSLs are a growing commercial industry, these cost reduction numbers should be reasonable.

DPPSL's easily meet the brightness requirements, by about two orders of magnitude or more. They also have the high contrast $\left(10^{4}: 1\right)$ needed for pulse shaping, and it appears that this is not an issue. However target designers can always come up with very strange pulse shapes (e.g., an exponential transition from foot to main pulse) that may present a challenge.

The present spatial uniformity of the DPPSL needs to be improved, but it is not known by how much. For example, although the present high-gain target design under development assumes the laser is very uniform throughout the entire pulse, it may be that high-beam uniformity is only required during the early foot. Nevertheless, a theoretically viable approach has been proposed, based on 2D-SSD, and sculpting the laser spectrum, and potentially four-color operation, to achieve very uniform beams throughout the pulse-as low as $0.03 \%$ RMS at $1 \mathrm{~ns}$, in modes $20-300$ is projected. However, this smoothing, if achievable, ignores the modes $<20$ which SSD cannot smooth as well, and comes at the expense of a very large number of beams, which may effect the cost, efficiency and durability. The Mercury laser will provide the necessary test bed to perform a proper integrated demonstration of this technology.

\section{Krypton Fluoride Laser (KrF)}

NRL is building the Electra $\mathrm{KrF}$ Laser $(700 \mathrm{~J} / 5 \mathrm{~Hz} / 30 \mathrm{~cm}$ aperture) to develop the technologies required for IFE. The requirements for an IFE $\mathrm{KrF}$ laser were derived from power plant studies and the high-gain target design. The present Nike amplifier ( 5 $\mathrm{kJ} / 38 \mathrm{~kJ}$ e-beam) suggests that scaling the architecture (ISI beam smoothing, angular multiplexing and double-sided e-beam pumping) to a IFE-sized laser (30-50 kJ, with eight $38 \mathrm{~kJ}$ e-beams), should be a reasonable extension of existing technology. The challenge is to build one with the needed rep-rate, durability, efficiency and cost.

The cost requirements are estimated to be $\$ 225 /$ laser Joule, of that the pulsed power costing $\$ 5.00 /$ lectron beam Joule ( $\$ 70 /$ aser Joule). It appears the pulsed power costs can be met, based on systems studies, vendor estimates based on anticipated technology advances, and economies of scale, but this needs to be demonstrated. The pulsed power system will be all solid state and will probably feature some form of magnetic compression as used in the Sandia RHEPP accelerator. The cost of the other laser components needs to be refined, but based on several studies (Sombrero, LANL, and Ignition facility) the overall system costs seems reasonable.

All the components of a $\mathrm{KrF}$ system need to be developed for durability, but the two most limiting components are the hibachi foil (which separates the laser gas at $\sim 1$ 
atmosphere from the diode at vacuum) and the amplifier optical window. Both last several hundred shots on Nike, and both need to have lifetimes exceeding $10^{8}$. It is anticipated that a long-life hibachi can be built using a helium cooled double foil arrangement as tested by AVCO. The window will require an optics development program.

$\mathrm{KrF}$ has demonstrated extremely high beam spatial uniformity: $1.2 \%$ nonuniformity per beam. This should be adequate for IFE. Based on other excimer lasers, this uniformity is expected to hold for a rep-rated system. KrF brightness is an order of magnitude higher than required for direct drive. Pulse shaping is not an issue, within the same "target designer" constraints as a DPSSL. One of the advantages of a KrF system is that the pulse shaping, zooming, and beam smoothing can be carried at low energy in a single front end which feeds the entire laser.

The present projected efficiency of a $\mathrm{KrF}$ laser is about 7\%. This is adequate for IFE, based on the Sombrero Power Plant studies, but leaves little head room. The efficiency estimates are based on systems studies of the advanced pulsed power $(80 \%)$, experiments with a high transparency hibachi developed at Los Alamos (80\%), the estimated efficiency of the ancillary components such as gas recirculator (95\%), the demonstrated intrinsic efficiency of $\mathrm{KrF}$ test cells, de-rated by $20 \%$ for fill factor (12\%), and beam transport to target $(90 \%)$. Needless to say, efficiency is one of the prime areas that will be addressed with Electra.

\section{Z pinches}

Z Pinches make lots of $\mathrm{x}$ rays; $1.9 \mathrm{MJ}$ and $200 \mathrm{TW}$, efficiently and at relatively low cost $\left(\$ 30 /\right.$ Joule $\left._{x \text {-ray }}\right)$. The Sandia $Z$ machine is an outstanding technical achievement, and the question is now whether or not the concept can be turned into a viable approach for fusion energy. This system is just now starting to be evaluated, and with no power plant concept to define the requirements, it would be difficult to answer the specific questions of this topic. Nevertheless, several points can be made regarding a z-pinch driver: first, $\mathrm{z}$ pinches are scalable to large drive energies (up to $60 \mathrm{MA}$ is considered) which will lead to large fusion yields. Hence the rep-rate, in principle, can be low (assuming other power plant issues allow this). Second, the efficiency of this system (from wall plug to x-ray energy out) has been demonstrated (single shot) to be about $15 \%$. It is not unreasonable to assume that future pulsed power developments can improve this two-fold. Third, pulse power has historically been inexpensive compared to other drivers, and, it is not unreasonable to assume cost reductions as the technology is improved. Fourth, the technology to rep-rate a pulsed power system has been demonstrated with the RHEPP machine at Sandia.

Whether all these attributes can be maintained as they are integrated into a fusion power system needs to be addressed. For example: the efficiency of the whole system (wall plug to fusion energy, including cooling and ancillary components) needs to be taken into account. The target design may require pulse shaping that can affect the driver characteristics. And, it will take a significant engineering effort to get to a rep-rated multi-TW system. To go from the RHEPP technology to the initial driver designs requires a $4 \mathrm{x}$ increase in voltage $(2.3 \mathrm{MV}$ to $10 \mathrm{MV})$, and a $2000 \mathrm{x}$ increase in current $(25$ $\mathrm{kA}$ vs. $50 \mathrm{MA}$ ).

\section{Other Concepts: Medium Weight Ions}

This talk addressed one question for one driver, namely how can the source brightness be improved for a light ion driver. The source under consideration is a cryogenically cooled extraction ion diode. It has achieved 3-6 milliradians divergence with a nitrogen beam (there is some oxygen impurity), as compared to 15-60 milliradians for proton beams. This "Medium Ion Beam" (MIB) approach combines the low cost of light ions, with the high brightness of Heavy ions. 


\section{Question 2B: What are the key standoff issues for each driver scenario and how can they be addressed? (e.g., final optics for lasers, final transport and focus for laser beams, final focus magnetic lenses for heavy ion beams, final transport and focus for ion beams, power feed for rep-rated $\mathrm{z}$ pinches,...)}

Session Leader: John Barnard

Speakers to initiate discussions:

HIF and LIF

DPSSL and $\mathrm{KrF}$

$\mathrm{Z}$ pinches

\author{
Craig Olson \\ Steve Payne and John Sethian \\ Steve Slutz
}

\section{Session Summary (John Barnard)}

The success of Inertial Fusion Energy (IFE) depends on the ability to transport a succession of pulses of energy to a small volume in a short time to detonate a sequence of targets, without the resulting target explosion destroying the chamber or the permanent structures used in delivering the pulse. "Standoff issues" are those physics and engineering issues that are related to the delivery of the energy pulse through the chamber and to the protection of the permanent structures. Some commonalities exist between the standoff issues for the various driver concepts, and these are outlined in this brief introduction. In the following sections, we focus on the standoff issues unique to ion drivers (both heavy- and light- ions), then on laser beam issues (DPSSL's and $\mathrm{KrF}$ ). Finally, the issues for the exploratory concepts (z-pinch and MTF) are described.

For ion and laser beams, the solid walls of the chamber must be protected from

neutrons, $\mathrm{x}$ and $\gamma$ rays, streaming gas and ions, and debris from the target explosion. In addition, the final focusing optics must be protected, since they are generally large, high precision, and costly components. In some designs, bending optics (which must also be protected) are used to introduce a jog in the laser or ion beam path. This jog permits a neutron and $\gamma$-ray dump to be inserted on a line-of-sight to (but further from) the target, reducing further transport of $\gamma^{\prime} s$ and neutrons up the ion or laser beam line. Shutters are often provided just outside the chamber to limit much of the projectile debris and gas flow from the target explosion. The density of vapor or gas in the chamber must be chosen to be consistent with propagation of the beam at high intensity.

For the z-pinch concept and some Magnetized Target Fusion concepts, it is required to have conductors deliver the energy pulse to the target; therefore, a cost-effective way of replacing the power feeds must be developed. In addition, the higher yields of these latter options must be considered in the chamber design.

\section{HIF and LIF}

The main standoff issues facing ion drivers are (1) the choice and validation of beam propagation mode in the chamber (that results in a sufficiently small and wellpointed spot at the target), (2) beam instabilities, and (3), for heavy ions, configuration of the final magnet assembly and shielding of the magnet. Because of the relatively small total beam solid angle and narrow cone angles required by a two-sided indirect drive target, flowing thick liquid walls (e.g. Flibe, LiPb, LiSn, or Li) provide an attractive means of protecting the solid chamber wall from neutron, $\gamma$ rays and $X$ rays from the target explosion, but other wall options (dry-wall and liquid-wetted) are available for ions (and have been described in system studies). The other chamber options are discussed in 
greater detail in the section on IFE chambers; here we will generally assume thick-liquid Flibe walls to discuss ion beam standoff issues.

There are a number of propagation modes that are being considered for transport of the ion beam through the chamber. They may be broadly divided into unneutralized ballistic transport, neutralized ballistic transport, and channel transport.

Unneutralized transport may be considered for chamber pressures less than $\sim 10^{-4}$ Torr, where generation of free electrons is sufficiently low. This pressure is in the lower end of the range contemplated for Flibe liquid walled chambers. Unneutralized transport is the mode of operation of most accelerators in operation today that require small focal spots, such as colliders. However, the currents required for heavy ion fusion are much larger so space charge effects become important. Unneutralized propagation is possible even, e.g., for the case of a target that requires $4 \mathrm{MJ}$ of $4 \mathrm{GeV}$, mass 200 ions, provided the number of beams is increased to 480 (up from the original design of 48 , where $90 \%$ charge-neutralization was required). For a beamlet focusing half-angle $\theta=0.01 \mathrm{rad}$, the total solid angle obtained by this number of beams would be $1.2 \%$ of $4 \pi$ steradians. Some current chamber designs prefer fewer beams, so targets which allow larger ion energies, or neutralized propagation modes, are favored. The issues associated with unneutralized ballistic focus would be few, however, if neutralized modes prove to be unsuccessful. One issue is the degree of stripping of the beam by the residual gas and the subsequent defocusing of the beam from space charge. Operating at the lower end of possible Flibe gas pressures ( $<10^{-4}$ Torr) reduces this effect. A second issue is the degree to which photoionization by the target $X$ rays (generated as a result of target heating by the prepulse) ionizes the surrounding gas and the beams. Since this occurs near the target and will likely provide partial beam neutralization, the effect on focusability could be beneficial. Both effects have been simulated but require examination over a wider parameter range in fully-integrated simulations.

For neutralized ballistic transport, there are a number of options for providing electrons to neutralize the beam, and minimizing the effects of space charge: preionization of the gas through which the beam propagates; co-injection of electrons; passage of the beam through a disposable foil or gas puff; or beam induced ionization of the gas. The light ion fusion program has experimentally achieved $99.98 \%$ neutralization by passage of the beam through a foil, confirmed in calculations by the hybrid electromagnetic code IPROP, so the more modest neutralization fractions (90-99\%) proposed for heavy ion fusion do not appear to be problematic. Neutralization fractions of $\sim 90 \%$ have been simulated using the electromagnetic code BICrz, (assuming a preionized surrounding cylindrical column exists) and have achieved nearly emittance-limited spot sizes. These simulations, however, neglected some electron sources (e.g. chamber walls, nearby Flibe, target emission and photoionization); the code

improvements to include these sources are in progress. Ionization methods including whistler mode wave heating will be experimentally investigated in the near term.

The final option, at higher risk, is that of channel transport whereby azimuthal magnetic fields provide strong focusing of the beam. The magnetic fields are generated by either a plasma discharge producing a longitudinal current (a "pre-formed channel" [ 10-100 Torr]); a wire carrying a current ("wire-guided"); or "self-pinched" transport (whereby the fields from a highly-stripped, but space-charge neutralized ion beam produce the self-focusing magnetic field [at $\sim 5-30 \mathrm{mTorr}]$ ). The main advantage of channel transport options are that the ion beams may be combined in a small number of entrance holes (reducing the total beam solid angle thus minimizing neutron and $\gamma$-ray leakage from the chamber). Also, the focal distance to the entrance hole can be much less than the chamber radius, easing beam brightness requirements. For preformed channels, creating and maintaining a stable channel, and establishing return current paths which, in some cases, can be geometrically orthogonal to the beam current path, are the key 
scientific issues. For self-pinched transport, recent experiments at NRL with high-current proton beams have demonstrated this mode, in the pressure regime predicted by IPROP, for the first time.

Instabilities may also be a concern. For unneutralized ballistic transport, the known beam instabilities are not expected to be of concern. However, as the neutralization fraction increases, in the neutralized ballistic and channel transport modes, filamentation, resistive hose, and the electron-ion two stream must be considered. The linear growth rates for these modes have been calculated. Simulation studies using BICrz have not detected any instabilities in the partially-neutralized regimes thus far simulated (with $\sim 90 \%$ neutralization fractions), but the code is axisymmetric. Channel transport modes have more complicated physics and the effects of instabilities are still being explored. Filamentation has been observed in experiments at NRL with proton beams in certain pressure regimes. The use of high-current $(-100 \mathrm{kA})$ proton beams to model stripped heavy ion beams was advocated.

In addition to instabilities, other physics questions such as those regarding the interaction of multiple beams, or the degree to which the longitudinal velocity tilt can be removed at the stagnation point, just before the beam passes the final optics, need to be addressed via both simulations and experiment.

For the mainline ballistic focus, the final focus quadrupole and bending magnets do not materially intersect the beam path. For efficiency reasons, low $\mathrm{T}_{\mathrm{c}}$ superconducting magnets (e.g., using $\mathrm{NbTi}$ cable at $4^{\circ} \mathrm{K}$ ) are the baseline case for final focusing and bending magnets. However, low $\mathrm{T}_{\mathrm{c}}$ magnets can tolerate only $1.0 \mathrm{~mJ} / \mathrm{cm}^{3}$ of heating before a quench (reversion to normal conducting) can occur. Shielding must therefore be provided to prevent scattered neutrons and $\gamma$ rays from intersecting and heating the coils of the magnets. Both longitudinal shielding (between the magnet and the target) and radial shielding (between the beamline and the magnetic coils) can be used. Simulations using neutron and $\gamma$-ray transport codes have shown that in simple examples enough shielding can be provided to prevent magnet quenching. The exact geometry of the final focus magnets is still being designed. How to compactly configure the quadrupoles as close as possible to the chamber and to each other, with adequate longitudinal and radial shielding, is an optimization question which is still being addressed. Other types of magnets such as high $T_{c}$ and even pulsed normal conducting magnets (for the final few magnets in the beamline) could be used, should low $\mathrm{T}_{\mathrm{c}}$ superconductors prove to be unattractive. If high $\mathrm{T}_{\mathrm{c}}$ magnets work and are economical, they will become the main approach rather than a backup.

Other issues include the control of gas flow up the beam lines. A combination of shutters and strong differential pumping is envisioned to prevent debris and gas from propagating down the beam line, adversely affecting beam propagation.

The questions posed in the previous sections will be addressed through a number of existing and upcoming experiments, together with sophisticated simulations. Scaled final. focus experiments at LBNL have demonstrated that emittance-limited spot radii are obtainable from space-charge dominated beams for unneutralized ballistic transport, and neutralized experiments using a grid are planned. The planned High Current Experiment (1 A) will allow similar studies at line.charge densities comparable to those in a driver (but at larger perveance). The NRL machine, Gamble II, has tested, and may test further, aspects of neutralized and channel transport modes using proton beams. The Integrated Research Experiments will enable definitive studies of several propagation modes and neutralization concepts at perveances and line charge densities near to or larger than in a driver. Simulations using the codes BIC, IPROP, and the latter's descendant version LSP will be carried out with realistic models for electron generation, permitting confidence in the understanding and extension of IRE-level results (100s $\mathrm{MeV})$ to driver-scale results $(1-10 \mathrm{GeV})$. 


\section{DPPSL and $\mathrm{KrF}$}

As mentioned above, the chamber, final bending and focusing optics must be protected from $x$ and $\gamma$ rays, from gas pressure impulses, and shrapnel from the exploding hohlraum. The mainline DPSSL and $\mathrm{KrF}$ approaches to IFE use direct drive targets that require laser lines of sight to the target distributed approximately uniformly in solid. angle. Liquid flows are therefore not favored for first wall protection and either dry walls or liquid wetted walls are the baseline designs.

If unprotected, the first wall would receive $\sim 0.5-5 \mathrm{~J} / \mathrm{cm}^{2} \mathrm{x}$-ray fluence per shot, ablating $\sim 0.1$ to $1 \mu \mathrm{m}$ per shot (depending on material) off the solid wall at a nominal 5 meter radius. For IFE, ablation must be limited to $\ll 1 \mathrm{~nm}$ per shot. To avoid this ablation, the chamber would be filled with $\mathrm{Kr}$ or Xe gas at $\sim 0.5$ Torr, reducing the $\mathrm{X}$-ray fluence by two orders of magnitude, and reducing the ablation rate to $\ll 1 \mathrm{~nm} / \mathrm{shot}$ as required. There are two constraints on the chamber gas density. Gas breakdown by the laser occurs if the laser intensity is $>10^{14} \mathrm{~W} / \mathrm{cm}^{2}$ at 1 Torr. Thus at 1 Torr, breakdown will not occur until the beams propagate to $\sim 1 \mathrm{~cm}$ of the target (but would suffer breakdown on target $\sim 4 \times 10^{14} \mathrm{~W} / \mathrm{cm}^{2}$ ). Experiments with Nike at $10^{14} . \mathrm{W} / \mathrm{cm}^{2}$ have shown little difference between a 0.1 Torr gas fill and vacuum propagation; experiments at 0.5 Torr show some changes, but these may be instrumental. The other constraint on the chamber gas is set by heating of the cryogenic target, which calculations show will occur if the pressure is greater than $\sim 0.5$ Torr. Experiments to explore target survivability in chamber gas have been proposed using wind-tunnels, in the near term, at a universityscale. Determining the exact pressure range where breakdown and target heating are avoided and wall protection is afforded is still a research area. Also the effects of impurities in the residual gas on breakdown need further investigation. Finally, the ablation rate has been estimated using Nova $\mathrm{x}$-ray results; these results may prove to be pessimistic as the $\mathrm{x}$-ray spectrum from the direct-drive target explosion differs considerably from the indirect-drive target experiments.

A second option for wall protection is to use wetted walls $(\mathrm{Pb})$ as in the Promethius design (1995). Breakdown occurs at a factor of two lower density than in $\mathrm{Kr}$, so $\sim 0.5$ Torr would be the maximum vapor pressure allowed during the laser pulse. Calculated recovery times indicate that the vapor pressure can be reduced to $\sim 0.1$ Torr between shots, well below the breakdown limit. To protect the final optic in the wetted wall reactor design, a $\mathrm{Kr}$ or Xe gas puff in a tube between the reactor wall and the optic could be used to provide the shielding from $\mathrm{x}$ rays. The fill time is $\sim 10 \mathrm{msec}$ and optical distortions have been experimentally shown to stabilize after $\sim 50 \mathrm{msec}$.

The final (bending) optic requires some protection. If a $\sim 0.5 \mathrm{Torr} \mathrm{Kr}$ or $\mathrm{Xe}$ gas fill is used to avoid first wall ablation, then at the final optic $(\sim 25 \mathrm{~m}$ from the target) the $x$-ray flux would be about 6 orders of magnitude lower than in the vacuum case. The dose rate in the final optic would be dominated by the line-of- sight neutrons. The final optic would bend the laser beam and allow residual line-of-sight neutrons and gamma rays to pass into a beam dump. There are several candidates for final optic: Grazing Incidence Metal Mirror (GIMM); hot fused silica prism or grating; also, other optical materials or liquid metal mirrors could prove useful. GIMM's have a laser damage threshold of 1 $\mathrm{J} / \mathrm{cm}^{2}$. GIMM's will accumulate $\sim 3$ displacements per atom (resulting in an acceptable $99 \%$ reflectivity for Aluminum GIMM's) after an exposure of $2.4 \times 10^{12}$ Rads (corresponding to an exposure of $2 \times 10^{4} \mathrm{Rad} / \mathrm{s}$ for 4 years at a standoff distance of $25 \mathrm{~m}$.) Thus, each GIMM would need to be replaced 7 to 8 times over the 30 year lifetime of the power plant. Additional work is needed on the optical damage threshold of GIMMS versus wavelength, and with neutron exposure.

The second option for an optical material is heated fused silica $\left(\mathrm{SiO}_{2}\right)$. This has a higher damage threshold $\left(>10 \mathrm{~J} / \mathrm{cm}^{2}\right)$ than GIMM's, although the effect of radiation on damage fluence needs further investigation. As the $\mathrm{SiO}_{2}$ accumulates neutron and 
gamma-ray dosage over time the absorption coefficient increases because of defects. Experiments on the SPRM nuclear reactor at Sandia National Laboratory have begun assessing the change of absorption coefficient as a function of radiation exposure. For the drive $(3 \omega)$ radiation of a DPSSL (350 $\mathrm{nm}$ wavelength), the absorbtion coefficient was few per cent after exposures of $320 \mathrm{kRad}$ neutrons and $800 \mathrm{kRad} \gamma$ rays obtained in 8 shots. The IFE rate is $18 \mathrm{krad} / \mathrm{s}$ in neutrons and $3 \mathrm{kRad} / \mathrm{s}$ in $\gamma$ rays, so the test was equivalent to $\sim 20$ seconds of IFE reactor neutrons. Annealing tests at $350^{\circ} \mathrm{C}$, showed the absorption coefficient between 200 and $300 \mathrm{~nm}$ to be significantly reduced after a few hundred seconds, and at $350 \mathrm{~nm}$ to be reduced somewhat. Numerical models which assume $50 \mathrm{kRad} / \mathrm{s}$ of neutron dose for temperatures-between 350 and 500 degrees $\mathrm{C}$ indicate that the absorption coefficient at $350 \mathrm{~nm}$ reaches a steady state $\left(0.04 \mathrm{~cm}^{-1}\right.$ for $500^{\circ} \mathrm{C}$ ). Thus, the self-annealing properties of the hot-fused silica could make an attractive solution to the final optic problem. Other materials such as KDP appear to be harder to neutrons than fused silica and therefore merit further investigation. However, this potential solution is only available to DPSSL's, since the absorptivity at the shorter wavelength of $\mathrm{KrF}$ would be too large, even with annealing. For DPSSL's, the long term effects of radiation on the damage threshold and absorption coefficient need study. Finally, the effects on final optic of residual debris from the target explosion that is not stopped by the shutter should be evaluated.

The final focusing optic in the beam line is located approximately $50 \mathrm{~m}$ from the target explosion. As a result of the final bending optic, the final focusing optic receives a radiation dose primarily from scattered radiation off of the bending optic. The contribution from radiation that penetrates the neutron dumps is small. The total radiation flux is $\sim 20$ times less than at the final bending optic, so that GIMM's or heated fused silica optics could again provide the solution.

\section{Z-pinch}

$\mathrm{Z}$ pinches provide a straightforward way for converting stored electrical energy in capacitor banks into $x$ rays which can drive a hohlraum. For the IFE application the main conceptual problem is how to deliver the energy pulse to the z-pinch repetitively in an economically attractive manner. There have been three conceptual approaches to the power feed problem: (1) recyclable transmission lines (RTL's), (2) flux compression by high velocity projectiles, and (3) ion or electron beams.

The most promising approach appears to be the RTL. Several ways have been proposed to construct the RTL. Wire webs, or sheets of material could be used to provide smooth anode surfaces. The material could be produced from stainless steel (at $\sim \$ 3 / \mathrm{kg}$ ), plastic coated with $\mathrm{Al}(\sim \$ 1 / \mathrm{kg})$, or solid coolant (e.g., Flibe [a salt] with a thin conducting coating, or solid lithium). The cost of material limits the mass of the RTL. For $1 \mathrm{GJ}$ of generated electricity, $\$ 19$ (at $\$ 0.07 / \mathrm{kwatt}-\mathrm{hr}$ ) of revenue are generated, limiting RTL mass to a few $\mathrm{kg}$ if composed of steel (for example). However, magnetic pressure in the RTL will result in electrode motion during the pulse, which results in electrode kinetic energy and increased RTL inductance. As the RTL mass is increased the electrode motion is reduced. Thus, there is a tradeoff whereby low RTL mass reduces cost, but increases RTL motion and thus decreases efficiency, and increases recirculating power. Estimates indicate that yields of 1-10 GJ will be required, with shot frequencies less than $\sim 1 \mathrm{~Hz}$ and standoff distances greater than $4 \mathrm{~m}$. Yields can be reduced if some of the excess magnetic field energy can be reused, e.g. by recharging capacitors with the reflected wave, but details need to be worked out.

The second option considered envisions a conical projectile to compress a conical electrode, within which an initial current has been seeded flowing in a poloidal direction. The projectile reduces the area enclosed by the current increasing the flux and driving a voltage. Ten $\mathrm{km} / \mathrm{s}$ projectiles have been demonstrated and would be envisioned for this 
application. The disadvantage of this concept is that the area of the projectile would be quite large $\left(\sim 500 \mathrm{~m}^{2}\right.$ for typical parameters). Also, large initial seed fields and some pulse compression would also be required.

The third option considered would use electron or ion beams to power the $\mathrm{z}$ pinch. In essence this requires a "reverse diode" whereby a beam is absorbed on a grid creating the potential ( $\sim 8 \mathrm{MV})$ to drive the current ( $60 \mathrm{MA})$ which drives the $z$-pinch. It may be difficult to generate and propagate such a low impedance electron beam; the possibility of current neutralization and ion stripping make ion beams more attractive. There are several issues of inverse diode operation that need to be studied. Diodes may require an initial magnetic field to keep electrons from traveling across the gap, although, it is expected that once current is delivered to the gap the self-magnetic field will perform this task. Instability-induced electromagnetic fluctuations may allow electrons to cross the gap even in the presence of a strong magnetic field, which would lower the efficiency of converting beam energy into current. However, this process also has the beneficial effect of charge neutralizing the beam within the gap allowing the use of a larger gap. Finally, the anode will become a source of ions which will be accelerated across the gap, thus requiring a clean high- $Z$ anode to reduce the parasitic current.

Clearly, IFE z pinches are in the concept exploration phase. More work is needed on a self-consistent reactor conceptual design, as well as more concrete RTL designs in the favored approach. Experiments on existing SNL.z-pinch machines would be useful in exploring some of the fundamental questions in this concept.

\section{MTF}

Magnetized Target Fusion operates in a density regime that is somewhere between MFE and IFE. However, some options for MTF have similarities with respect to the chamber and standoff issues for IFE. One concept analogous to IFE, requires the injection of a $\sim 10 \mathrm{~cm}$ diameter, DT gas filled metal shell into an IFE-like chamber. A conducting helical $\mathrm{Li}$ wire connects the top and bottom of the shell, which are separated by an insulating ring. A current through the wire is generated using charged ion beams (one positively and one negatively charged, or possibly ion and electron beams) at the poles of the shell, which create a Field Reversed Configuration (FRC) magnetic field interior to the metal shell. This allows a lower compressed line density pr than required for IFE, requiring a lower implosion velocity. The shell is imploded using particle, laser, or plasma beams that compress and ignite the fuel. The standoff issues associated with this concept may have commonalities with ion or laser driven fusion, as beams are candidates for both magnetic field generation and ablatively driven implosion of the shell. However, the regimes are quite different than IFE, in that pulse lengths are on the order of 1 to $10 \mu$ s and the beam energies are in the 10-50 MJ, with target yields in GJ range. The exact concept for the driver needs more development.

There are other options for MTF such as magnetic, or pneumatic pressure-driven implosions, and some options under consideration may use power feed options similar to those envisioned for $\mathrm{z}$ pinches. As with $\mathrm{z}$ pinches, self-consistent reactor concepts for MTF are in an early stage and need further development. Proof of principle experiments on high density FRCs using single shots and liner implosions are being considered to address basic feasibility issues of MTF.

\section{Question 2C: What would convincingly demonstrate that each driver concept is a viable driver candidate for IFE? Specifically, what is a convincing Integrated Research Experiment (IRE) for each driver candidate?}

Session Leader: Rick Spielman

Speakers to lead discussions: 


$\begin{array}{ll}\text { HIF } & \text { Roger Bangerter } \\ \text { LiF } & \text { Mike Cuneo } \\ \text { DPSSL } & \text { Howard Powell } \\ \text { KrF } & \text { John Sethian } \\ \text { Z-pinch } & \text { Steve Slutz }\end{array}$

\section{Session Summary (Rick Spielman)}

\section{Definition of an IRE}

The first thing that this question raised was the need to clarify (at least to the participants) the IFE road map. The participants pointed out the need for clear milestones and metrics for all levels of the road map. The suggestion was made that motion from one level of the road map to another should be based on universal performance metrics.

There were spirited and candid discussions on the area of metrics and milestones concerning how one defines key parameters such as cost and efficiency needed for an IRE. One real question is demonstrated vs. projected performance. Also, there was a difference of opinion on what level of performance an IRE needed to have. The DPSSL and $\mathrm{KrF}$ teams argued that an IRE should demonstrate all of the technology and physics of the driver by building a single module of a fusion driver. This would validate driver technology, transport performance, and cost. The Heavy Ion team argued that only scaled experiments are necessary and that an IRE need not have the parameters of a single module of a fusion driver only the results need be scaled to reactor driver parameters. A Heavy Ion IRE would have a lower voltage, a lower current, a different ion, and could not conduct full-scale transport experiments. The definition of an IRE needs to be settled prior to a decision on moving forward on the road map.

\section{HIF IRE.}

HIF supporters described their roadmap to an IRE as based on experiments from a number of facilities around the world and from calculations. The HIIF IRE is a facility to validate the metrics of $\mathrm{HIF}$ and to make engineering and physics improvements. It was also suggested that the HIF IRE be a flexible research tool. The proposed HIF IRE has the following proposed performance parameters: $\mathrm{Kr}^{+1}$ ions, $200-\mathrm{MeV}$ energy, $1 \mathrm{kA}$ current, a total beam energy of $30 \mathrm{~kJ}$, and a $335 \mathrm{~ns}$ pulse length bunching to $5 \mathrm{~ns}$. A summary of the issues presented is given below:

(1) Scaled beam experiments have been performed with brightness exceeding the requirements for a fusion driver. Other beam requirements are met with scaled dimensionless parameters.

(2) The ETF/DEMO driver requirements determine the IRE goals. This includes beam quality, cost, beam neutralization, etc.

(3) The cost goal of $\$ 150 \mathrm{M}$ for the IRE requires improvements in the state-of-the-art of accelerator technology.

(4) At a minimum the IRE must validate the expectation of beam coupling. Exploring other target issues is desirable.
1. Beam target physics at $3 \times 10^{12} \mathrm{~W} / \mathrm{cm}^{2}$
2. Focussing experiments
3. Beam dynamics
4. HIF target concepts

\section{DPSSL IRE}

The DPSSL program optimism is based on recent laser technology development and direct-drive target experimental results. The DPPSL testbed "Mercury" was initiated with DP/LDRD funding at LLNL. With $100 \mathrm{~J}$ of energy, Mercury will be able to validate 
many of the laser physics and engineering assumptions that are being made today, during a Phase $1(\mathrm{PoP})$ program.

Because of the extensive work in the area of Nd:glass laser technology the real issues that impact DPSSL's are those relating to the DPSSL components and amplifiers needed for high rep rate. Also the cost of DPSSL's are now at $\sim \$ 3.00$ per W or $6 \mathrm{x}$ higher than needed for an IRE.

Mercury's goals are:

a. Demonstrate efficiency

b. Test gas cooled amplifiers

c. Test new laser crystals

d. Test rep rated architecture

e. Demonstrate beam quality

f. Demonstrate efficient frequency conversion

Mercury will not test full size apertures.

The IRE, if the Phase 1 goals are met, would address integrated performance, segmentation issues within a beam line, vendor development, beam transport, optics survivability, target tracking, and materials tests. Cost is the major issue for DPSSL's, but it is not unreasonable to expect reductions in cost as commercial applications of DPSSL's grow. The cost of a DPSSL IRE is estimated to be $\$ 50 \mathrm{M}-\$ 100 \mathrm{M}$, following a $\$ 40 \mathrm{M}$ Phase 1 (PoP) program.

\section{KrF Laser IRE}

The next step in the $\mathrm{KrF}$ laser program is the construction of a Phase 1 (PoP) laser called "Electra" by CY 2003. This laser would be "relatively small but would demonstrate most of the key technologies needed for the IRE. The Electra parameters are planned to be: $5 \mathrm{~Hz}$ rep rate, $30-\mathrm{cm}$ aperture, and $700 \mathrm{~J}$. The key Phase 1 issues are:

a. Rep rate

b. Durability

c. Beam quality

d. Cost

e. Efficiency

f. Continued IRE systems studies

The proposed IRE performance parameters are: 150-kJ energy, $\quad \eta \sim 6-7 \%, 5 \mathrm{~Hz}$, $3 \times 10^{8}$ shots $\left(10^{10}\right.$ lifetime), $\$ 225 / \mathrm{J}$ costs, with a total IRE cost of $\$ 50 \mathrm{M}-\$ 100 \mathrm{M}$. The key issues that must be successfully addressed before proceeding to an IRE are efficiency and lifetime.

\section{Z-pinch IRE}

$\mathrm{Z}$ pinches are a new concept for IFE. The immediate attraction of the concept is the low cost of the driver and the high efficiency of converting stored electrical energy to $\mathrm{X}$ rays $(15 \%)$. Recyclable transmission line concepts have been proposed to solve the standoff issue; these were discussed in the Standoff Session and in the IFE Power Plants Session.

The z-pinch IFE concept is presently in the Concept Exploration Phase and would be expected to remain there for a few years. Note that z-pinch physics issues and ICF issues are being address through DP funding. Besides standoff, the major deliverable prior to moving toward a PoP Phase would be a comprehensive reactor system study. Phase 1 (PoP) deliverable would address rep rate in pulse power and stand off at the 0.1 $\mathrm{Hz}, 20-\mathrm{MA}$ level.

\section{LIF IRE}

LIF is a long time IFE concept that has been unable to demonstrate adequate nonprotonic beam intensity. Recent analyses have shown that this was primarily due to the 
lack of a pure ion source. Low-level Concept Exploration work has been proposed that would focus on the ion source issue. Subsequent Phase 1 work could be accomplished on existing Sandia pulsed power devices (RHEPP 1, RHEPP 2, HERMES III). The key metric for proceeding to Phase 1 would be the demonstration of a pure ion source. The advantages of efficiency, cost, and an attractive power plant scenario with standoff are the impetus for continuing the effort.

\section{IRE Summary Comments}

The presentations for the driver candidates gave their individual plans leading to an IRE. The laser presenters (DPSSL and $\mathrm{KrF}$ ) suggested that they are now beyond the Concept Exploration phase and are ready to proceed with Phase 1 Proof-of-Principle $(\mathrm{PoP})$ experiments. In the case of heavy ions, which are currently engaged in PoP development, the argument was made that the next step is an IRE. The other concepts, $\mathrm{Z}$ pinches and light ions, remain in the Concept Exploration phase.

\section{Subgroup 3: Inertial Fusion Power Plant Concepts Ken Schultz, Robert Peterson, Per Peterson}

\section{Inertial Fusion Power Plant Concepts Summary (Ken Schultz)}

An IFE Power Plant concept is the integrated choice of a target, a driver, a reaction chamber, and a heat transport/power conversion system. The target options are direct or indirect drive, and the specific target designs are strongly driven by the physics requirements and the choice of driver, although target fabrication and injection issues, compatibility with the chamber design and environmental issues must also be considered. Options for the driver to heat and ignite the target are heavy ion beams, lasers, light ions and pulsed power sources such as $\mathrm{z}$ pinches. Three major categories have been explored for chambers to contain the target explosions and convert the $\mathrm{x}$-ray, debris and neutron energy from the explosion to heat: dry-wall designs, wetted-wall designs, and thick liquid-wall concepts. For each of these options a variety of material choices and configurations have been explored. Heat transport and power conversion systems that have been considered include flowing liquids (Flibe, $\mathrm{LiPb}, \mathrm{Li})$, flowing solids $\left(\mathrm{Li}_{2} \mathrm{O}\right.$ ) and various coolants (He, $\left.\mathrm{H}_{2} \mathrm{O}\right)$. Rankine (steam) and Brayton (gas turbine) power conversion cycles have been considered.

Each driver has a choice of target and chamber options, and the characteristics of the drivers appear well suited to selected options. The heavy ion beam driver is wellsuited to indirect-drive targets and the thick liquid wall chamber concept, for example, the HIILIFE-II design. The high efficiency of the heavy ion accelerator accommodates lower indirect-drive target gains, and the short standoff of the liquid chamber concepts eases beam focus requirements. Wetted-wall chambers like OSIRIS also match well with heavy ion beam drivers. Laser beam drivers like the $\mathrm{KrF}$ or solid state lasers are well suited to direct drive and dry-wall chamber designs like SOMBRERO. The higher gain of direct-drive targets eases concerns about low laser efficiencies, and the longer stand-off of lasers allows use of a larger, simple chamber with reduced concern about final optic protection. The wetted-wall chamber concept is also possible with laser beam drivers, as exemplified by the KOYO design. The "main line" IFE power plant concepts thus appear to be settling down to two: the heavy ion beam driver with indirect-drive targets and a thick liquid-wall chamber, and a laser beam driver with direct-drive targets and a drywall chamber, both with a wetted-wall chamber as a "back-up" option.

Several IFE "Exploratory Concepts" also exist that either have identified technical hurdles or that have been little studied, but should be kept in mind. Light ion beam 
drivers offer the potential for high efficiency and low cost, but have been unable to overcome ion source limitations. Ideas exist for ion sources (possibly with "middle weight" carbon ions) that may overcome this limitation. The wetted-wall. chamber concept appears well-suited to light ion fusion. The "fast ignition" concept involves using "conventional" target implosion to compress a cold target to high density and a separate igniter beam of extreme intensity to initiate a propagating fusion burn in the compressed target. The physics of laser interaction with matter at these extreme conditions are little explored, but if fast ignition proves to be real, it offers the intriguing possibility of very high target gains $(\sim 500)$.for low input energy $(\sim 500 \mathrm{~kJ})$, which could make possible both an improved fusion power plant product, and a lower cost development pathway. Depending on the target and driver configuration, fast ignition. might use dry-wall, wetted-wall or liquid-wall chamber concepts. However, a key unexplored issue is delivering the fast ignitor beam in the chamber environment. The z-pinch has made tremendous progress in the past year and offers large $x$-ray energy with high efficiency and low cost. Concepts exist for using this energy as a driver for inertial fusion and, if successful, might be rep-rated to form the basis for an IFE power plant. This potential driver, and the related Magnetized Target Fusion concept which uses a liner to compress a magnetically confined plasma, could use a thick liquid-wall or possibly solid Li with voids and a density gradient for shock dissipation.

Clearly, there are several viable target options for IFE. The proper choice varies from driver to driver, and each driver could be coupled with several chamber options. The major technical issues for IFE target chambers can be divided into four areas: chamber dynamics, chamber materials, liquid hydraulics, and neutronics, safety, and environment. The Snowmass participants discussed these areas in depth, identifying the key technical issues. Many of the technical issues can be investigated in existing ICF or MFE facilities and programs, or in small-scale "university type" laboratory experiments, thus allowing progress in IFE chamber development to proceed with modest funding.

Issues exist for IFE target fabrication and injection that must be resolved for IFE to be a practical energy alternative. For indirect-drive HIF IFE, target fabrication is the main issue. Significant design and development work is needed on fabrication of the distributed radiator hohlraum/beryllium capsule target. For laser direct-drive IFE, target survival during injection is the main issue. Thermal protection of the capsule is essential and will require serious re-evaluation of the dry-wall target chamber concept.

IFE chambers and targets are key features of an IFE power plant. Numerous issues exist that must be resolved for IFE to be a practical energy alternative. However, numerous possible solutions also exist that appear attractive. It is essential that close coordination be established between the target designers, the chamber developers, the target fabricators, the target injection system designers, and the safety and environment worriers. The basic approach that is recommended is to take maximum advantage of the work that is being done by the ICF Program for the NIF. In parallel, we must carry out IFE design studies on selected issues and modest scale laboratory development activities to demonstrate and select the appropriate options. This effort is expected to result in the demonstration that a credible pathway exists to practical IFE chamber design and target fabrication and injection with a high probability of success. This information will contribute to the decision in about 2003, or later, of whether or not to proceed with an IFE IRE and if so, what technologies to use. These will then be applied to the IRE, demonstrating many of the chamber and target technologies needed for IFE. Concepts.

Following are summaries of the three sessions on Inertial Fusion Power Plant

Question 3A: What are the key IFE power plant concepts, advantages, and issues?

Session Leader: Robert Peterson 
Speakers to initiate discussions:

HIF power plant concepts

LIF power plant concepts

Wayne Meier

DPSSL and $\mathrm{KrF}$ power plant concepts

Z-pinch power plant concepts

MTF power plant concepts

Robert Peterson

Gerald Kulcinski

Mark Derzon

Kurt Schoenberg

\section{Session Summary (Robert Peterson)}

This session addressed the question of what are the major design concepts, what are their advantages and key issues. There was some question as to the meaning of "key". One interpretation is issues that can invalidate the concept or effect its attractiveness as a power plant concept. This session was organized as a series of talks by advocates of different types of IFE power plants arranged by driver type, with ample time for discussion and questions. The discussions were generally constructive in pointing out issues and suggestions for how a concept could be improved. There were some members of the MFE community present. As a result of the presentations and discussions, a list of issues was defined. We have organized the issues and driver type into Table 9. Based on the discussions during the sessions some elements in this matrix are defined: others are not.

Wayne Meier presented a summary of Heavy Ion Fusion power plants, which consisted of one thick-liquid design and three wetted-wall designs. The advantages of thick liquid protection (HYLIFE-II) are the plant structure lifetime, the need for a greatly reduced materials program, the projected low cost of electricity and low tritium inventory. There are unresolved issues of liquid jet hydraulics, chamber dynamics, driver cost, Flibe dissociation, corrosion and cleanup, the lifetime of hardware, tritium control, and diagnostics. It is clear from this list that, even though HYLIFE-II is currently the best funded IFE power plant concept development effort, it has more issues than can be addressed in the current program. The attractive features of the wetted-wall concepts (HIBALL, OSIRIS, Prometheus-H) are lower pumping powers, rapid replacement of flow control devices, low activation, resistance to $x$-ray and debris damage, and the possibility of adaptability to laser fusion. Issues include fabricability of wetted structures, wetting mechanisms, flow around penetrations, rep-rate, maintenance and tritium recovery. The list of issues is substantial and there are currently no efforts on wetted wall concepts in the US. It was suggested that both thick-liquid and wetted-wall concepts could benefit from new data on PbLi, Flibe and SnLi.

Bob Peterson presented a summary of Light Ion power plants. Work on LIF has been placed on hold in the US because of ion source problems and the growing interest in $\mathrm{z}$ pinches, although it could be revived with perhaps a different ion species. Beam transport issues were thought to be the most difficult issue in the LIBRA series of LIF power plants, so each was designed around a particular transport method: LIBRA used preformed channels; LIBRA-LiTE used neutralized ballistic; and LIBRA-SP used selfpinched channels. All three concepts are wetted walls and have similar chamber issues to

Table 9. IFE Power plant concepts.

\begin{tabular}{|l|l|l|l|l|l|l|l|l|}
\hline Type & $\begin{array}{l}\text { Plant } \\
\text { Concept }\end{array}$ & $\begin{array}{l}\text { Stand-off } \\
\text { HIF }\end{array}$ & $\begin{array}{l}\text { Driver } \\
\text { Cost }\end{array}$ & $\begin{array}{l}\text { Driver } \\
\text { Energy }\end{array}$ & $\begin{array}{l}\text { Target } \\
\text { Yield }\end{array}$ & $\begin{array}{l}\text { Driver } \\
\text { Eff. }\end{array}$ & $\begin{array}{l}\text { Thermal } \\
\text { Eff. }\end{array}$ & Materials \\
\hline $\begin{array}{l}\text { HIBALL } \\
\text { OSIRIS } \\
\text { Prometh.-H }\end{array}$ & $\begin{array}{l}\text { HI } \\
\text { transport }\end{array}$ & $\begin{array}{l}\text { High } \\
\text { Cost } \\
\text { code }\end{array}$ & $\begin{array}{l}\text { Low- } \\
\text { Med. }\end{array}$ & $\begin{array}{l}\text { Low- } \\
\text { Med. }\end{array}$ & High & Med. & $\begin{array}{l}\text { Flibe/SS } \\
\text { PbLi/SiC } \\
\text { Flibe/C } \\
\text { Pb/SiC }\end{array}$ \\
\hline $\begin{array}{l}\text { LIBRA } \\
\text { LIBRA-LiTE } \\
\text { LIBRA-SP }\end{array}$ & LI transport & $\begin{array}{l}\text { Low } \\
\text { Expert } \\
\text { costing }\end{array}$ & Med. & Med. & High & Med. & $\begin{array}{l}\mathrm{PbLi/SiC} \\
\text { Li/SS } \\
\text { PbLi/SS }\end{array}$ \\
\hline
\end{tabular}




\begin{tabular}{|c|c|c|c|c|c|c|c|c|}
\hline & $\begin{array}{l}\text { ADLIB } \\
\text { UTLIF }\end{array}$ & & & & & & & \\
\hline $\begin{array}{l}\text { Laser } \\
\text { Fusion }\end{array}$ & $\begin{array}{l}\text { SOLACE } \cdot \\
\text { HYLIFE } \\
\text { SOMBRERO } \\
\text { Prometh.-L }\end{array}$ & $\begin{array}{l}\text { Mirrors/ } \\
\text { Lens } \\
\text { Survival }\end{array}$ & $\begin{array}{l}\text { Med. } \\
\text {-High } \\
\text { Cost } \\
\text { code }\end{array}$ & $\begin{array}{l}\cdot 1-4.5 \\
\mathrm{MJ}\end{array}$ & $\begin{array}{l}150- \\
1800\end{array}$ & $\begin{array}{l}5 \%- \\
12 \%\end{array}$ & $\begin{array}{l}39 \%- \\
55 \%\end{array}$ & $\begin{array}{l}\mathrm{LiO}_{2} / \mathrm{C} \\
\mathrm{Li}_{\mathrm{SS}} \\
\mathrm{LiAlO}_{2} / \mathrm{SiC} \\
\mathrm{PbLi} / \mathrm{SiC}\end{array}$ \\
\hline Z-Pinch & - & $\begin{array}{l}\text { Recycled } \\
\text { Trans. Line }\end{array}$ & Low & High & $\begin{array}{l}\text { Very } \\
\text { high }\end{array}$ & High & $?$ & $\mathrm{Li} / \mathrm{SS}$ \\
\hline MTF & - & $=$ & Low & High & $\begin{array}{l}\text { Very. } \\
\text { high }\end{array}$ & High & $?$ & $\mathrm{Li}$. \\
\hline Type & $\begin{array}{l}\text { Wall } \\
\text { Protection }\end{array}$ & $\begin{array}{l}\text { Materials } \\
\text { Recycle }\end{array}$ & $\begin{array}{l}\text { Target. } \\
\text { Inject. }\end{array}$ & $\begin{array}{l}\text { Develop } \\
\text { Cost - }\end{array}$ & ES\&H & Avail. & $\begin{array}{l}\text { Unit } \\
\text { Size - }\end{array}$ & Maintain. \\
\hline$\overline{\text { HIF }}$ & $\begin{array}{l}\text { Thick liquid } \\
\text { Wetted wall }\end{array}$ & - & $\begin{array}{l}\text { pneuma } \\
\text { tic }\end{array}$ & $\begin{array}{l}\text { IRE } \\
\text { ETF }\end{array}$ & $\begin{array}{l}\text { Flibe } \\
\text { Low } T_{2}\end{array}$ & - & $\begin{array}{l}2-4 \\
\text { GWe }\end{array}$ & Good \\
\hline$\overline{\mathrm{LIF}}$ & Wetted wall & - & $\begin{array}{l}\text { pneuma } \\
\text { tic }\end{array}$ & - & - & - & $\begin{array}{l}300- \\
1000 \\
\mathrm{MWe}\end{array}$ & Good \\
\hline $\begin{array}{l}\text { Laser } \\
\text { Fusion }\end{array}$ & $\begin{array}{l}\text { Thick liquid } \\
\text { Gas } \\
\text { Granular }\end{array}$ & - & $\begin{array}{l}\text { pneuma } \\
\text { tic }\end{array}$ & $\begin{array}{l}\text { NIF } \\
\text { IFE } \\
\text { ETF }\end{array}$ & $\begin{array}{l}\mathrm{C} \\
\text { High. } \mathrm{T}_{2}\end{array}$ & - & $\begin{array}{l}700- \\
1000 \\
\text { MWe }\end{array}$ & Good \\
\hline Z-Pinch & Thick liquid & - & $\begin{array}{l}\text { With } \\
\text { MITL }\end{array}$ & $\begin{array}{l}Z \\
Z X X \\
X-1\end{array}$ & - & - & 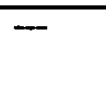 & - \\
\hline MTF & Thick liquid. & $=$ & - & $=$ & - & - & $\overline{-}$ & - \\
\hline
\end{tabular}

the wetted wall HIF concepts. The allowed gas density in the chamber is generally high in the LIF concepts so rep-rate issues are not as extreme. The unique issues of LIF power plants are beam generation and transport. The advantages of LIF are high efficiency, lowcost pulsed power, and the demonstrated rep-rate of ion drivers. There is currently no effort in the US on LIF power plants, but some of the ideas could be applied to other IFE.

Jerry Kulcinski summarized the state of laser fusion power plants. Because of the relatively low efficiency of $\mathrm{KrF}$ drivers, the higher-gain direct-drive targets were chosen over indirect-drive. This led plant designs away from thick-liquid chamber concepts. Fear of liquid condensation on laser optics led the designs to gas-protected walls. Gas protection involves the use of a gas that is dense and thick enough to stop the most penetrating ions and $x$ rays from reaching the target chamber wall. The SOMBRERO design uses 0.5 Torr of Xe gas in a $6.5 \mathrm{~m}$ radius chamber to stop $1.6 \mathrm{MeV} \mathrm{C}$ ions from the ablator of the target. A fireball is formed in the gas by the $\mathrm{x}$-ray and ion energy deposition that radiates energy to the graphite wall over about $0.1 \mathrm{~ms}$; a time that is long enough for conduction in the graphite to keep the temperature low enough to avoid erosion of the wall. The critical issues of this concept are the target emissions, the fireball behavior, the first wall heat conduction, tritium retention in the wall, and target injection through the gas. The advantages of gas protection are no vapors to condense on the laser optics and the ability to handle many penetrations. Prometheus- $L$ is a wetted wall concept for lasers. Liquid metal grazing incidence mirrors would allow some vapor to be in the target chamber, though most laser designers would prefer that no vapor was present. Tritium retention is a major safety issue, but it was pointed out that high temperatures in the graphite of SOMBRERO would reduce the tritium retention. New two-sided illumination targets would lead to new chamber concepts. Finally magnetic protection of the wall was suggested as a way of reducing the chamber gas density.

Mark Derzon summarized the sate of $z$-pinch drive IFE power plants. $Z$ pinches have the major advantage of being tolerant of dirty chamber conditions. The concept, so far un-named, uses re-cycled Flibe and $\mathrm{Al}$ transmission lines to carry current to the target. This standoff issue is quite different from HIF, LIF and lasers in that there is no transport required through gas. The transmission lines and target are rapidly inserted into a $\mathrm{Li}$ pool with steel walls. The blast, which would be of high yield, is contained in the liquid, which 
is dumped into the basement after the shot. There it is fed into heat exchangers, tritium extraction and materials recycling equipment. The concept is new, so there are many issues yet to be resolved, but the fact that it does not require a pristine chamber environment avoids problems that are a constant worry in other concepts. Several suggestions were made for improvements to the concept, such as bubbles in the liquid to mitigate shocks. Z-pinch fusion could also have a less expensive development path because of the low cost of pulse power.

The final talk was by Kurt Schoenberg on magnetized target fusion. This talk was more on the promise of MTF than on a specific power plant concept. MTF combined some advantages of IFE and MFE. MFE can be explored on existing or soon to exist machines without the need for a dedicated facility. Two old power plant concepts for liners (FLR and Linus), traditional IFE chamber concepts, and the SNL z-pinch power plant concept may all be adaptable to MTF. There is a wide range of target yields that might be attractive for a power plant. Attractive power plants are possible at lower gain than usual for IFE. Target fabrication may also be easier.

In summary, there are several viable target chamber options for IFE. The proper choice varies from one driver type to another, and in fact each driver type could be made to work with multiple chamber options. There are many issues for each of the chamber options, because the IFE chamber technology program has been very small. It is hoped that a new IFE chamber technology program will be developed that will begin to address some of these issues systematically. A new technology program should balance resolution of issues on older concepts with development of new ones. Care should be taken to keep the three main concepts (thick liquid, wetted-wall, and gas-protected) in the development path. Magnetic protection and granular flows should also be kept in play. It has been many years since a new IFE power plant concept has been developed in the US. New technologies and new driver and target constraints have appeared in the mean time. Therefore, there should be some effort into updating old concepts and developing new concepts.

\section{Question 3B: What are the key scientific issues for the fusion chamber (e.g., first wall protection, ....) and what are the proposed solutions? What experiments could be done to test the relative merits of these solutions?}

Session Leader: Per Peterson

Speakers to initiate discussions: Chamber concepts summary Chamber dynamics for IFE Chamber materials for IFE Liquid hydraulics Neutronics, activation, safety Chamber development path

\author{
Mark Tillack \\ Robert Peterson \\ Everett Bloom \\ Per Peterson \\ Jeff Latkowski \\ Wayne Meier
}

\section{Session Summary (Per Peterson)}

The IFE target chamber must provide the interface between the target, the drive, the blanket and the balance of plant. The chamber concept selection and design will have major leverage on the attractiveness of an inertial fusion energy power plant. Target chamber systems for inertial fusion energy (IFE) must:

- regenerate chamber conditions for target injection, driver beam propagation, and ignition at sufficiently high rates (i.e. $3-7 \mathrm{~Hz}$ );

- protect chamber structures for several to many years (ideally the life of the plant, -30 yr.) or allow easy replacement of inexpensive modular components; 
- extract fusion energy in a high-temperature coolant and produce tritium; and

- reduce the volume of radioactive waste generation and reduce possible release fractions low enough to meet no-public-evacuation standards.

Because the target chamber is a relatively small fraction of the total capital cost of an IFE power plant $(9 \%-12 \%)$, the target chamber influences the cost of electricity primarily through the driver-target coupling, controlling the required driver energy; through the plant availability and safety, and through controlling waste generation. Beyond making IFE technically and economically feasible, target-chamber research provides the potential for substantial leverage to reduce the IFE cost of electricity.

Driver standoff provides a major input for chamber technology selection. Because laser beams can be focused accurately at long distances and because laser final optics rely on standoff to control $\mathrm{x}$-ray and debris damage, laser IFE chamber concepts have gravitated toward dry-wall designs, as shown in Fig. 2, which can produce minimal debris mobilization. Solid walls introduce issues related to neutron irradiation damage, which can be controlled by optimizing the chamber size, taking advantage of the reduction of neutron fluence obtained with the square of chamber radius, and by use of rapidly replaceable blanket structures and re-use of the blanket breeding material. Heavy ion drivers, light ion drivers, $\mathbf{z}$ pinches, and magnetized target fusion (MTF) perform best when the standoff distance is minimized. This provides motivation to pursue thick-liquid chambers, where liquid jets can be located within tens of centimeters of targets to provide shielding of chamber structures (Fig. 2). The time required for gravity clearing of the chamber after a shot can pose a rep-rate limit, which can be circumvented by using oscillating flow streams to sweep the chamber, dynamically clearing it of droplets from the previous shot. For all of the driver options, wetted-wall chambers with flow-guiding structures provide an intermediate option, simplifying liquid hydraulics questions at the expense of higher neutron damage to the flow structures and, for lasers, at the expense of potential optics contamination from liquid evaporation.

The major technical issues for IFE target chambers can be divided into four technical subtopics: Chamber Dynamics, Chamber Materials, Liquid Hydraulics, and Neutronics, Safety and Environment. The Snowmass participants discussed these areas at depth, identifying the key technical issues summarized in Table 10. Many of the technical issues can be investigated in existing ICF or MFE facilities and programs, or in small scale, "university type" laboratory experiments, thus allowing progress in IFE chamber development to proceed with modest funding. 


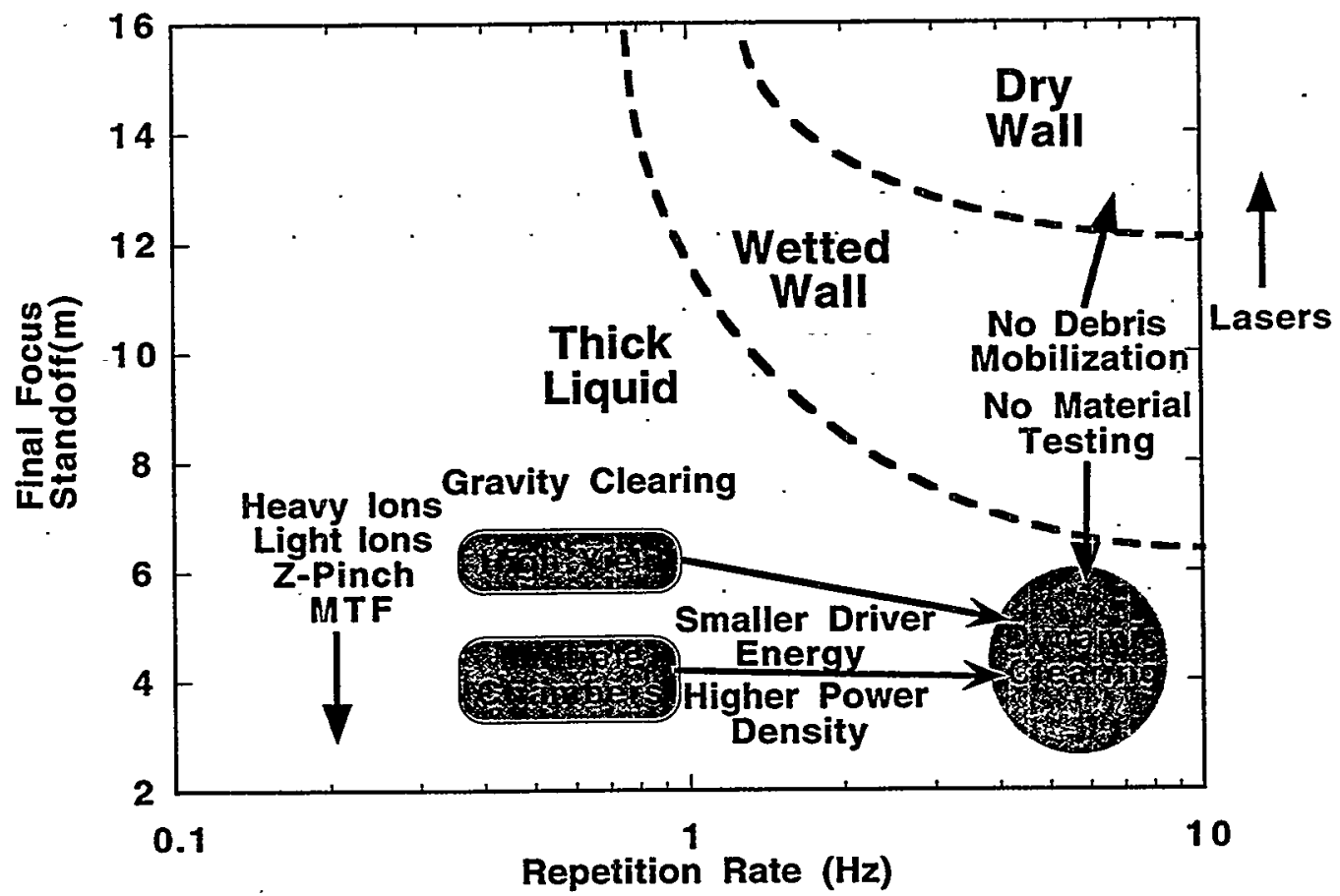

Figure 2. Driver stand-off characteristics will influence chamber selection.

Table 10. IFE Target Chamber Issues (Possible near-term approaches in parenthesis).

\begin{tabular}{|c|c|c|c|}
\hline Research Area & Thick Liquid & Wetted Wall & Dry Wall \\
\hline $\begin{array}{l}\text { Chamber } \\
\text { Dynamics }\end{array}$ & \multicolumn{2}{|c|}{$\begin{array}{l}\text { Target induced impulse loads to liquid } \\
\text { Condensation of target and ablation debris by droplet } \\
\text { sprays } \\
\text { (Z-pinch, university experiments) }\end{array}$} & \begin{tabular}{|l|} 
Direct-drive target \\
emission \\
Fireball re-radiation or \\
magnetic diversion of \\
target ions \\
(Z-pinch experiments) \\
\end{tabular} \\
\hline Chamber Materials & \multicolumn{2}{|c|}{ Corrosion, hohlraum material recovery from coolant } & \begin{tabular}{|l} 
Fusion neutron effects on \\
structures \\
(materials development \\
parallels MFE \\
efforts)
\end{tabular} \\
\hline Liquid Hydraulics & $\begin{array}{l}\text { Formation of free jets } \\
\text { Pocket disruption and } \\
\text { droplet clearing } \\
\text { (water experiments with } \\
\text { scaled impulse loads) }\end{array}$ & $\begin{array}{l}\text { Liquid film formation and } \\
\text { stability } \\
\text { (small-scale experiments) }\end{array}$ & \\
\hline $\begin{array}{l}\text { Neutronics/ } \\
\text { Safety/ } \\
\text { Environment }\end{array}$ & \multicolumn{3}{|c|}{$\begin{array}{l}\text { 3-D modeling of final focus neutron and gamma irradiation } \\
\text { Hohlraum, coolant and structure materials activation } \\
\text { Accident mobilization and off site dose minimization } \\
\text { Waste minimization } \\
\text { (mobilization experiments with liquid coolants) }\end{array}$} \\
\hline
\end{tabular}

Question 3C: What are the issues in target fabrication, target characterization, 
target injection, target robustness (e.g., tolerances), and what is the path for addressing them?

Session Leader: Ken Schultz

Speakers to initiate discussions:

Target physics requirements

Target fabrication issues

Target injection issues

Possible development paths

Max Tabak and Debra Callahan-Miller

Warren Steckle

Gottfried Besenbruch and Ron Petzoldt

Ken Schultz

Session Summary (Ken Schultz).

The goal of these discussions was to consider the target fabrication and injection requirements for the power plant concepts being developed for both direct-drive and indirect-drive IFE, and to identify the pros, cons, issues and opportunities associated with each concept. We hope that the results of these discussions will contribute to the eventual selection of a limited subset of these target concepts and development of their fabrication and injection technologies for application to IFE.

This topic had strong interaction with the Targets Subgroup. This Session of the Inertial Fusion Power Plants Subgroup focused on what to do for IFE target fabrication and injection, and the companion afternoon IFE Target Technologies topic group focused its discussions on how to do IFE target fabrication and injection.

\section{Description}

At the heart of an inertial fusion explosion is a target that has been compressed and heated to fusion conditions by the incident driver energy beams. For direct drive, the target consists of a spherical capsule that contains the DT fuel. For indirect drive, the capsule is contained within a cylindrical or spherical metal container or "hohlraum" which converts the incident driver energy into $\mathrm{x}$ rays to drive the capsule. The "Target Factory" at an inertial fusion power plant must produce about 1-2 $\times 10^{8}$ targets each year, fill them with deuterium-tritium fuel, layer the fuel into a symmetric and smooth shell inside the capsule, and deliver the completed target to the target chamber at a rate of about $5 \mathrm{~Hz}$. These fragile targets must be injected to the center of the target chamber, operating at a temperature of $500-1500^{\circ} \mathrm{C}$ and possibly with liquid walls, without mechanical damage from handling and acceleration, or thermal damage from the hot target chamber environment. Target fabrication must be done with extreme precision of manufacture, extreme reliability of delivery and at a manufacturing cost four orders of magnitude lower than current ICF target fabrication experience. Target injection must be done with precision, and reliability of delivery and without damaging the mechanically and thermally fragile targets. The choice of power plant concept, chamber design and protection scheme, and target design all impact the target fabrication and injection challenges. These challenges do appear to be achievable, but will require a serious - and successful - development program.

\section{Target Physics Requirements}

Debbie Callahan-Miller described the physics requirements, materials and design options, and current designs for indirect drive HIF targets. Both direct and indirect drive targets were also discussed thoroughly during the IFE Targets subgroup of the IFE Concepts sessions.

The current leading direct-drive target design promises high gain $(\sim 130)$ and consists of a $\mathrm{CH}$ capsule with low density $\mathrm{CH}$ foam inside and a thin high-Z coating outside. The foam is $~ 100-250 \mu \mathrm{m}$ thick, is filled with solid DT and surrounds an additional $\sim 100-200 \mu \mathrm{m}$ of pure DT. This design appears simple and should be fabricable 
by techniques already explored in the ICF target fabrication program. However, there is still some physics uncertainty associated with the design and the fabrication tolerances (foam cell size and allowed material, density and dimensional variations) have not been explored. The thermal protection of this target may be favorable in that it has a thin outer layer of high- $Z$ material that could potentially be highly reflective to thermal radiation. However, the fabrication technique for, and properties of this layer, have not been determined.

The current leading candidate design for indirect-drive HIF targets utilizes a low density distributed radiator in the hohlraum walls to convert heavy ion beam energy to the $\mathrm{x}$ rays that compress the capsule. It promises high gain $(-50-130)$ by use of a "closecoupled" design with the hohlraum fitting closely around the capsule, and a beryllium capsule. Concern was expressed over how to fabricate the low density, high- $Z$ radiator materials, which do not have an ICF target analog. Callahan-Miller pointed out that a number of alternate material choices are possible and suggested that a number of techniques might work to achieve the low density, including foams, fibers and foil layers. Dimensional tolerances need to be defined, but are almost certainly less stringent than those of capsules, and no more strict than what must be achieved for the NIF. The beryllium capsule is similar to capsules being developed for the NIF, but low cost fabrication techniques and low tritium inventory fill techniques for mass production have not been identified. A high density plastic, such as polyimide, could be substituted for the beryllium ablator for a modest increase in driver energy.

\section{Target Fabrication}

Ken Schultz pointed out that targets currently fabricated for ICF experiments have many of the characteristics that will be needed for IFE, although the size is smaller (capsule diameter $\sim 0.5 \mathrm{~mm}$ for Nova, $\sim 1 \mathrm{~mm}$ for Omega and $\sim 2-3 \mathrm{~mm}$ for the NIF vs. -4-5 mm for IFE). The current ICF target fabrication techniques were not intended to be particularly well-suited to economical mass production of IFE targets. For a power plant to be economically competitive, the target cost for a $\sim 500 \mathrm{MJ}$ yield target must be $\leq \$ 0.30$. However, some of the current ICF fabrication processes do extrapolate well to economical IFE and design studies suggest alternatives for those that do not. Specific target fabrication techniques were discussed further during the afternoon IFE Target Technologies sessions.

All current IFE target designs require low density components of various kinds for the converters, hohlraums or capsules. A potentially attractive way to achieve these low densities is to use foam materials for these components. Warren Steckle reviewed the many possibilities for materials composition, morphology and configuration that are available with foam materials. The next logical steps in fabrication of IFE targets is to understand the physics design requirements, select the materials and develop the fabrication processes.

Target fill and layering are important steps in the target fabrication process that will have a large impact on the overall attractiveness of IFE power plants. Gottfried Besenbruch discussed these issues as part of the afternoon IFE Target Technologies sessions. Targets for ICF experiments are filled by permeation and a uniform DT ice layer is formed by a process known as "beta layering". By use of very precise temperature control, excellent layer thickness uniformity and surface smoothness of about $1 \mu \mathrm{m}$ RMS can be achieved. These processes are suited to IFE, although the long fill and layering times needed may result in large (up to $\sim 10 \mathrm{~kg}$ ) tritium inventories. Permeation filling of beryllium capsules may be particularly challenging.

DT layer smoothness is a potential performance limitation for IFE. The smoothness needed for NIF indirect drive plastic ignition targets appears to be very close to the limits of smoothness that can be achieved by beta layering. If IFE targets need DT ice smoothness better than $\sim 1 \mu \mathrm{m}$ to achieve high gain, new layering techniques will be needed, such as the infra-red and microwave heating techniques that have so far shown 
about a factor of 2 improvement in DT ice surface smoothness. Since IFE targets have thicker DT ice layers than NIF ignition targets, they may be more tolerant of ice roughness.' Use of polyimide or beryllium capsules may relax the surface smoothness required by factors of several. Since the ignition curve is very sensitive to smoothness, a small variation in surface roughness might make a large difference in target gain. If the target gain is significantly reduced or if it is highly variable from shot to shot, this would be a performance limitation for IFE.

\section{Target Injection and Tracking}

Preliminary design studies of target injection for both direct-drive and indirectdrive IFE power plants were done as part of the SOMBRERO and OSIRIS studies completed in early 1992 . The direct-drive SOMBRERO design proposed a light gas gun to accelerate the cryogenic target capsules enclosed in a protective sabot. After separation of the sabot by centrifugal force, the capsule would be tracked using cross-axis light sources and detectors, and the laser beams were steered by movable mirrors to hit the target when it reached chamber center. Target steering after injection was not proposed. The indirect-drive OSIRIS design proposed a similar gas gun system for injection and crossed dipole steering magnets to direct the beams.

Ron Petzoldt summarized the work done to develop injection and tracking systems. A gas gun indirect drive target injection experiment was done at LBNL. The results showed that relatively simple gas gun technology could repeatable inject a non-cryogenic simulated indirect drive target to within about $5 \mathrm{~mm}$ of the driver focus point, within the range of laser or beam steering mechanisms to hit, but not sufficient to avoid the need for beam steering to achieve the $\pm 200 \mu \mathrm{m}$ accuracy needed. The LBNL experiment also demonstrated target tracking and real time target position prediction which approximately meets the $\pm 200 \mu \mathrm{m}$ accuracy needed. Experiments with the same gas gun using a simple magnetically separated sabot showed simulated room temperature direct drive targets could achieve the same placement accuracy. Direct drive will require $\pm 20 \mu \mathrm{m}$ target tracking and beam steering accuracy. The choice between beam steering and target steering should be studied.

Thermal protection of the cryogenic targets as they are injected into the high temperature target chamber is a serious concern for IFE power plants. For indirect drive, the hohlraum will provide adequate thermal insulation for the capsule. For direct drive, reflective coatings will reduce the radiation heating of a target and are actually part of the current leading direct drive target design. The SOMBRERO dry-wall chamber used 0.5 Torr of Xenon gas to reduce the rate at which $\mathrm{x}$-ray and plasma energy would be deposited on the chamber wall surface. This kept the chamber surface temperature excursion below the value for which significant ablation would occur. However, chamber gas at this pressure is sufficient to change the position of a direct drive target by order of $20 \mathrm{~cm}$ and require in-chamber target tracking. With high reflectivity targets and gas filled chambers, the majority of the target heating will be from the gas rather than from radiation. We do not have a reliable model for the gas heating of a target in the pressure, temperature, and target speed regimes we expect to operate in. The reduced $\mathrm{x}$-ray emissions from current low-z target designs may reduce the requirement for a protective gas in the chamber and should be evaluated. We also do not know with certainty what temperature profile will be required to avoid changes in the target that would reduce target gain. These are two important areas for near term study. A wetted chamber wall design such as was used in OSIRIS, Prometheus and Koyo could eliminate the need for a chamber gas. The use of magnetic fields to divert the charged particle debris from the wall should also be investigated.

Development pathway

IFE Target Fabrication and Injection are part of the IFE Roadmap. Ken Schultz summarized the current plans. Activities are divided into Phase I, planned over the next 4 
years (FY00-03), and Phase II, planned for the following 9 years (FY04-12) when the IFE Integrated Research Experiment(s) (IRE) will be built and operated.

During Phase I, we will carry out the following tasks to support the decision as to whether to proceed with the IFE IRE, and if so, what target technologies to use:

Target Fabrication

- Work with target designers and chamber developers to select promising target designs that optimize target gain, robustness and cost.

- Develop materials for IFE target requirements, such as robust foams, doped ablators and distributed converter hohlraums for HIF.

- Develop mass production techniques by reviewing and identifying suitable industrial technologies (such as microencapsulation, fluidized bed coaters, injection molding of hohlraum parts, etc.), demonstrating on the lab bench that they can achieve the accuracy needed, and projecting that they can meet IFE coat goals.

- Develop statistical quality control characterization concepts.

Target Injection

- Work with target designers and chamber developers to select promising target and chamber designs and to define their injection requirements.

- Select, design and develop the target injection systems best suited for direct drive and indirect drive targets.

- Demonstrate injection and tracking of simulated targets at room temperature.

- Measure the thermal response of cryogenic targets and demonstrate methods for thermal protection in the laboratory.

During Phase II of the IFE Roadmap, we will carry out the following tasks:

Target Fabrication

- Carry out bench scale tests of IFE target production processes.

- Evaluate proposed and alternative processes for accuracy, reliability/repeatability and cost.

- Provide prototype targets for experiments on the IRE.

Target Injection

- Add cryogenic target capability and a high temperature surrogate target chamber to the Phase I injector-tracking system for injection experiments.

- Provide a target injection-tracking system to the IRE for integrated system experiments.

The cost of this IFE Target Fabrication and Injection development activity, which is part of the IFE Chamber and Target Element of the OFES Virtual Laboratory for Technology, is estimated to be approximately $\$ 3 \mathrm{M} / \mathrm{yr}$ for 4 years (FY 2000 - FY 2003) to complete Phase I. The costs for Phase II can be estimated when the driver choice(s) for the IRE have been made.

\section{Conclusions}

IFE target fabrication, fill, layering, injection and tracking are key features of an IFE power plant. Numerous issues exist that must be resolved for IFE to be a practical energy alternative. However, numerous possible solutions also exist that, at least on paper and in design studies, appear attractive. For indirect-drive HIF IFE, target fabrication is the main issue. Significant design and development work is needed on fabrication of the distributed radiator hohlraum/beryllium capsule target. For laser direct- drive IFE, target survival during injection is the main issue. Thermal protection of the capsule is essential and will require serious re-evaluation of the dry-wall-target chamber concept. It is essential that close coordination be established between the target designers, the chamber developers, the target fabricators and the target injection system designers. The basic approach that is recommended is to take maximum advantage of the target fabrication, fill and layering work that is being done by the ICF Program for the NIF. In parallel, we 
must carry out IFE design studies on specific components and issues, and modest scale laboratory development activities to demonstrate and select the appropriate options. This effort is expected to result in the demonstration that a credible pathway exists to practical IFE target fabrication and injection with a high probability of success. This information will contribute to the decision in about 2003 of whether or not to proceed with an IFE IRE and if so, what technologies to use. These will then be applied to the IRE, demonstrating many of the target fabrication and injection technologies needed for IFE.

\section{Subgroup 4: IFE Metrics and Development Paths}

(Wayne Meier, John Perkins)

\section{IFE Metrics and Development Paths Summary (Wayne Meier)}

This session of the IFE working group covered two related topics: the IFE development path for various driver options and the metrics by which options are judged to be ready to advance to the next stage of development.

Following are summaries of the two sessions on Metrics and Pathways.

\section{Question 4A: What are the metrics for an entire IFE system for each step of development (e.g., concept exploration, proof of principle, performance extension, fusion energy development, DEMO, attractive commercial fusion power plant)? How are these incorporated into the IFE Road Map? How do we insure that there is a mechanism in place for new concepts to initiate a development path?}

Session Leader: John Perkins

Speakers to initiate discussions:

FESAC Metrics

Deliverable metrics, IFE Road Map and mechanisms for new concepts

Charles Baker

John Perkins

\section{Session Summary (John Perkins)}

The IFE "roll-back" road map and metrics were discussed in an IFE plenary session. Critical issues were posed as four questions: What is the proposed development path that rolls back from the attractive power plant? What are the objectives at each stage of the road map? (attractive reactor, DEMO, ETF, IRE, PoP, CE). What are the decision/performance metrics that permit concepts to be promoted from stage to stage?

How do we formally accommodate new initiatives/innovations into the development path at the exploratory concept level for both advanced physics and technology? To resolve critical issues, it was proposed to direct an IFE "tiger team" to condense present goals into a unified, concise "bible" containing objectives and decision metrics for each stage of the development path for all concepts.

Regarding new concepts, a possible formalism for accommodating new ideas at the Concept Exploration level was suggested. A possible plan might have:

(1) New funding starts every year with a recognized date for calls and submissions; reviewed every year.

(2) Competition for seed money in one of two tiers, say, $\$ 50 \mathrm{k}-\$ 300 \mathrm{k}$, and $\$ 300 \mathrm{k}-\$ 3 \mathrm{M}$.

(3) Strict peer review (including an additive "power plant implications" metric). 
(4) A 3 or 4 year lifetime with a rolling horizon. After this time, project competes for programmatic funding.

(5) Program solicits innovative proposals on advanced physics and advanced technology and reactor paradigms.

(6) Consider the DOE Laboratories' LDRD IR\&D program as a possible model.

\section{Question 4B: What is the status and development path of each present IFE scenario?}

Session Leader: Wayne Meier

Suppliers of discussion material on:

Target design

HIF development

DPSSL development

$\mathrm{KrF}$ development

LIF development

Z-pinch development

Target fab/injection

Chamber technology
Max Tabak, Debra Callahan-Miller, Jill Dahlburg, Rick Olson

Roger Bangerter

Howard Powell

John Sethian

Mike Cuneo

Rick Spielman

Ken Schultz

Wayne Meier

\section{Session Summary (Wayne Meier)}

\section{Development Paths}

Information was presented on the status of various development plans for target design, different driver candidates, chamber technology, and target fabrication and injection. Key issues and near term plans were discussed for each of these areas. We also identified opportunities for cost-effective development which was one of the objectives of the Snowmass Summer Study.

\section{Status of Plans}

Over the past year significant effort has gone into developing a integrated $R \& D$ plans for heavy ion and laser (both DSSL and $\mathrm{KrF}$ ) driven IFE. These include definition of near term (3-4 years) tasks for the drivers, chambers, target fabrication and injection systems, and target design work for both indirect-drive and direct-drive targets needed by these approaches. The estimated cost of this work is $\$ 50 \mathrm{M}$ per year.

At the concept exploration level, for z-pinch, LIF, and other concepts, little detailed planning has been done since these programs are not currently funded. Preliminary plans for the most important near term tasks were presented at this meeting.

\section{Issues}

Top level issues are summarized in Table 11. More extensive discussions of issues occurred in the subtopic groups on targets, drivers, and power plants, but it was generally agreed that this list was a reasonable summary of the key issues in each area.

\section{Opportunities for Cost-Effective Development}

For each element of the IFE program, an effort was made to identify characteristics or procedures that could contribute to cost effective development of IFE. These are summarized in Table 12. Perhaps one of the most important features is that the driver technologies can be developed in a modular way, by demonstrating a single beamline (for lasers or light ions) or single key components of the heavy ion driver. 
Another important opportunity longer term, is the ability to operate an engineering test facility (ETF) at modest scales. Chambers can be tested at about 1/10 full yield, and it was also argued that the heat transfer systems could be developed at about $1 / 10$ or $300 \mathrm{MW}_{\mathrm{t}}$.

Table 11. Top Level Issues for IFE

\begin{tabular}{|c|c|}
\hline Area & Issues \\
\hline Target Design & $\begin{array}{l}\text { High gain - experiments and calculations to validate } \\
\text { Interface issues (materials, spot size, illumination geometry) }\end{array}$ \\
\hline $\begin{array}{l}\text { Drivers } \\
\text { Heavy ions } \\
\text { DPSSL } \\
\text { KrF } \\
\text { Light ions } \\
\text { Z-pinch }\end{array}$ & $\begin{array}{l}\text { Cost / beam quality (focusability) } \\
\text { Cost / beam smoothing } \\
\text { Efficiency / durability } \\
\text { Source / emittance growth / durability / focusability } \\
\text { High rep-rate, recyclable MTL / integrated power plant concept }\end{array}$ \\
\hline $\begin{array}{l}\text { Chambers / Final } \\
\text { Optics }\end{array}$ & $\begin{array}{l}\text { HIF final transport } \\
\text { Clearing for liquid wall chambers } \\
\text { Lifetime for dry-wall chambers ' } \\
\text { Laser optics design / survivability ( } x \text { rays, debris, neutrons) } \\
\text { HI final magnet/chamber interface (configuration/heating/activation) }\end{array}$ \\
\hline $\begin{array}{l}\text { Target } \\
\text { Fabrication \& } \\
\text { Injection }\end{array}$ & $\begin{array}{l}\text { Low cost fabrication process / tritium inventory } \\
\text { Accurate, reliable injection } \\
\text { Target survival (mechanical and thermal loads) }\end{array}$ \\
\hline $\begin{array}{l}\text { Safety \& } \\
\text { Environmental }\end{array}$ & $\begin{array}{l}\text { Designs to meet no evacuation criteria / } \mathrm{T} \text { inventory / wastes } \\
\text { Recycling of target material }\end{array}$ \\
\hline
\end{tabular}


Table 12. Opportunities for Cost-Effective Development

\begin{tabular}{|c|c|}
\hline Area & Opportunities \\
\hline Target Design & $\begin{array}{l}\text { Leverage off ICF work } \\
\text { High gain at low driver energy for low energy ETF } \\
\text { Fast ignitor }\end{array}$ \\
\hline Drivers & $\begin{array}{l}\text { Demonstrate end-to-end technology at single beamline scale for lasers } \\
\text { Test single, full-scale components (e.g., cores) of ion drivers } \\
\text { Utilize / upgrade existing facilities (e.g., pulsed power machines) }\end{array}$ \\
\hline Chambers & $\begin{array}{l}\text { HII final transport experiments and simulations } \\
\text { Scaled hydraulic tests with water } \\
\text { Sub-scale tests at ETF level } \\
\text { Reduce neutron source requirements (fluid walls) } \\
\text { Synergy with MFE on materials development and qualification }\end{array}$ \\
\hline $\begin{array}{l}\text { Target } \\
\text { Fabrication \& } \\
\text { Injection }\end{array}$ & $\begin{array}{l}\text { Leverage off of ICF fab work } \\
\text { Develop / test injector with non-cryo systems to begin }\end{array}$ \\
\hline $\begin{array}{l}\text { Integrated } \\
\text { Systems } \\
\text { (e.g., IRE, ETF) }\end{array}$ & $\begin{array}{l}\text { Do integrated target injection and chamber tests with IRE prior to ETF } \\
\text { Beam switching to test multiple chambers } \\
\text { Vary rep-rate and yield to test at low power } \\
\text { Develop breeding blankets, heat recovery at modest scale }(\sim 300 \mathrm{MWt})\end{array}$ \\
\hline $\begin{array}{l}\text { Safety \& } \\
\text { Environmental }\end{array}$ & Synergy with MFE on analytical tools, data, etc. \\
\hline
\end{tabular}




\section{Distribution:}

1 MS 1190 J. P. Quintenz, 1600

1 MS 1188 C. Olson, 1600

1 MS 1194 D. H. McDaniel, 1640

5 MS $1194 \quad$ R. B. Spielman, 1644

1 MS $1191 \quad$ M. K. Matzen, 1670

1 MS 1193 J. L. Porter, 1673

1 MS 1193 M. E. Cuneo, 1673

1 MS 1186 T. A. Mehlhorn, 1674

1 MS $1196 \quad$ R. J. Leeper, 1677

1 MS $1196 \quad$ R. E. Olson, 1677

2 MS 1186 S. A. Slutz, 1674

1 MS 0839 G. Yonas, 16000

2 MS 0839 M. S. Derzon, 16000

1 MS 9018 Central Technical Files, 8940-2

2 MS 0899 Technical Library, 4916

1 MS 0612 Review and Approval Desk, 14912 For DOE/OSTI 